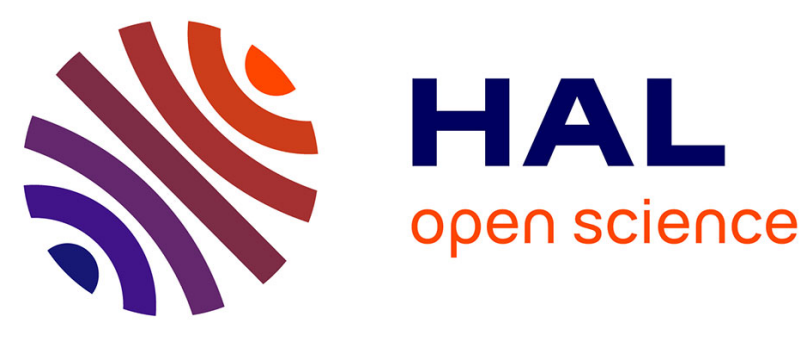

\title{
Synthesis of new dicinnamoyl 4-deoxy quinic acid and methyl ester derivatives and evaluation of the toxicity against the pea aphid Acyrthosiphon pisum.
}

Xiubin Li, Lucie Grand, Thomas Pouleriguen, Yves Queneau, Pedro da Silva, Yvan Rahbé, Jean-Luc Poëssel, Sylvie Moebs-Sanchez

\section{To cite this version:}

Xiubin Li, Lucie Grand, Thomas Pouleriguen, Yves Queneau, Pedro da Silva, et al.. Synthesis of new dicinnamoyl 4-deoxy quinic acid and methyl ester derivatives and evaluation of the toxicity against the pea aphid Acyrthosiphon pisum.. Organic \& Biomolecular Chemistry, 2016, 14 (8), pp.2487-2497.

10.1039/c5ob02483h . hal-01263941

\section{HAL Id: hal-01263941 \\ https://hal.science/hal-01263941}

Submitted on 15 Mar 2016

HAL is a multi-disciplinary open access archive for the deposit and dissemination of scientific research documents, whether they are published or not. The documents may come from teaching and research institutions in France or abroad, or from public or private research centers.
L'archive ouverte pluridisciplinaire HAL, est destinée au dépôt et à la diffusion de documents scientifiques de niveau recherche, publiés ou non, émanant des établissements d'enseignement et de recherche français ou étrangers, des laboratoires publics ou privés. 


\section{ARTICLE}

Received 00th October 2015,

\footnotetext{
a. Université Lyon 1, INSA Lyon, ICBMS Equipe Chimie Organique et Bioorganique UMR 5246 CNRS, CPE-Lyon, Bâtiment Jules Verne, 20 Avenue Albert Einstein, F69621 Villeurbanne Cedex, France; Tel : +33-(0)4 72438098 ; Fax : +33-(0)4 72 4388 96; E-mail : sylvie.moebs@insa-lyon.fr

b. INSA Lyon, INRA BF2I UMR0203, Biologie Fonctionnelle Insectes et Interaction, Bâtiment Louis Pasteur, 20 Avenue Albert Einstein, F-69621 Villeurbanne Cedex, France; Tel : +33-(0)4 724361 51; Fax : +33 (0)4 72438534 ; E-mail : pedro.da silva@insa-lyon.fr

c. INRA Avignon, GAFL, Domaine St Maurice, 84143 Montfavet Cedex, France; Email : jean-luc.poessel@avignon.inra.fr

† Footnotes relating to the title and/or authors should appear here.

Electronic Supplementary Information (ESI) available: [Copies of ${ }^{1} \mathrm{H}$ and ${ }^{13} \mathrm{C}$ NMR spectra for compounds $\mathbf{5 - 1 2},{ }^{1} \mathrm{H}$ NMR $\left(\mathrm{CD}_{3} \mathrm{OD}\right)$ spectra of $\mathbf{4 a}$ and $\mathbf{4 d}$ after different times intervals, HPLC chromatograms and UV spectra for tested compounds, as well as complementary data on survival assays are given in the supplementary information file]. See DOI: 10.1039/x0xx00000x
} 


\section{ARTICLE}

Accepted 00th January 2016

DOI: $10.1039 / x 0 \times x 00000 x$

www.rsc.org/

\section{Synthesis of new dicinnamoyl 4-deoxy quinic acid and methyl ester derivatives and evaluation of the toxicity against the pea aphid Acyrthosiphon pisum}

Xiubin Li, ${ }^{a}$ Lucie Grand, ${ }^{a}$ Thomas Pouleriguen, ${ }^{a}$ Yves Queneau, ${ }^{a}$ Pedro da Silva, ${ }^{b}$ Yvan Rahbé, ${ }^{b}$ Jean-Luc Poëssel, ${ }^{c}$ Sylvie Moebs-Sanchez, ${ }^{* a}$

New dicinnamoyl (caffeoyl, feruloyl, ortho and para-coumaroyl) 4-deoxyquinic acid and esters were synthesized by using a new 4-deoxy quinic acid triol intermediate. The optimisation of both coupling and deprotection steps allowed the preparation in good yields of the target products either as the carboxylic acid or the methyl ester form. Eight new compounds were evaluated for their ability to influence the feeding behaviour of the pea aphid Acyrthosiphon pisum. Artificial diet bioassays showed that two compounds are toxic (mortality and growth inhibition) at lower concentrations than the reference 3,5-dicaffeoyl quinic acid. 


\section{Introduction}

Chlorogenic acid, namely 5-O-caffeoyl quinic acid ${ }^{1,2} \mathbf{1}$ is the parent component of a large family (CQA : Cinnamoyl Quinic Acids) of natural compounds formed by esterification of D-quinic acid $\mathbf{2}$ with one or several trans-cinnamic acids such as, most commonly, caffeic, $p$-coumaric or ferulic acids (Figure 1). Dimethoxycinnamic ${ }^{3}$ and sinapic acids ${ }^{4}$ can also be present in some species. These bioactive plant secondary metabolites are widely found in edible plants, isolated from various species ${ }^{5,6}$ and have been commonly used in traditional herbal medicines. Suspected to have many health benefits, they have been widely evaluated in different in vitro bioassays to confirm their potential biological activities, among antioxidant, ${ }^{7}$ antifungal, ${ }^{8}$ antiviral, ${ }^{9}$ renoprotective, ${ }^{10}$ neuroprotective, ${ }^{11,12}$ antiatherosclerotic, ${ }^{13}$ or inhibitors of $\alpha$-glucosidase. ${ }^{14}$ They have also been recently included in diverse cosmetic compositions. ${ }^{15,16}$

Aphids are the major group of phloem feeding insects impairing seriously the production of agricultural and horticultural crops, causing significant yield losses and transmission of virus. Towards a more sustainable but efficient crop protection, which would prevent the development of insecticide resistance, the potential toxicity and environmental impact, alternative solutions to current pesticides are still investigated. Although host plant resistance to aphids mechanisms are not yet well understood, research for a biological mode of action could be an efficient alternative strategy. ${ }^{17}$ Indeed, the correlation between chlorogenic acids concentration and priming of plants $^{18}$ was shown for the carrot fly larval damage ${ }^{19}$ then for fungal infection ${ }^{8}$ or for cultured tobacco cells ${ }^{20}$ as examples.<smiles>O=C/C=C/c1ccc(O)c(O)c1</smiles><smiles>O=C(O)[C@]1(O)C[C@@H](O)[C@H](O)[C@H](O)C1</smiles>

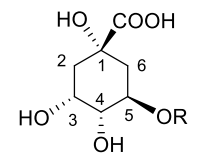

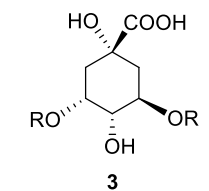

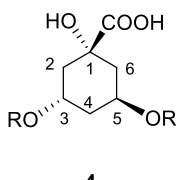

Figure 1 Representative structures of chlorogenic acid 1, D-quinic acid 2, dicaffeoyl quinic acid $\mathbf{3}$ and a deoxy analog 4

The resistance of lettuce cultivars to Pemphigus bursarius $^{21}$ or the negative effect of chlorogenic acid on

Western flower thrips Frankliniella occidentalis were also reported. ${ }^{22}$ More recently, the cultivar Rubira peach tree, parent of a trait mapped to $\mathrm{Rm} 2$ gene $^{23}$ showed a strong induced resistance to Myzus persicae, the green peach aphid which represents a threat for many agro-industrial crops including potato, sugar beet, tobacco or horticultural crops or stone fruits. ${ }^{24}$ In an early phenolic profiling study, ${ }^{25}$ an accumulation of metabolite later identified as 3,5dicaffeoyl quinic acid $\mathbf{3}$ had been detected in aphid-infested leaves, as was also later observed in a fungal-pear tree pathosystem. ${ }^{26}$ In spite of the positive preliminary results of in vitro artificial diets assays adding 3 in nutritive medium for aphids, ${ }^{27}$ the underlying biological mechanisms remain to be explored. A synthetic program was then initiated to prepare various dicinnamoyl quinic acid derivatives (DiCQA) and synthetic analogs to get insight into the pharmacophoric groups, into the mode of action of this phenolic compound onto putative specific aphicidal targets and/or to find more active compounds.

Due to known isomerization of $\mathbf{3}$ into the other 3,4 and 4,5 diCQ regioisomers under bioassay conditions, ${ }^{27}$ in organic solvents, ${ }^{28}$ or under very basic conditions, ${ }^{29}$ questions about the stability of compounds to synthesise were addressed. We hypothesized that the synthesis of $\mathbf{4}$ and derivatives, as first part of our program, as analogues of 3 and other CQAs, featuring a 4-deoxy quinic acid nucleus (Figure 1), would prevent the easy isomerization of 3,5derivatives. Here we wish to report our results on the first synthesis of new homo diesters 4 of 4-deoxy quinic acid, from the homo bis coupling of a new 4-deoxy triol with caffeic, coumaric and ferulic counterparts. The evaluation of the respective toxicity of 7 deoxy quinic acids or methyl esters derivatives against aphids was also carried out in comparison with the natural 3,5-diCQA derivative $\mathbf{3}$ and an analog prepared from isosorbide, a bicyclic diol.

\section{Results and discussion}

\section{Chemistry}

Our retrosynthetic analysis was logically inspired by the previous syntheses of chlorogenic acids. Except for an elegant and original methodology using a Knoevenagel reaction, ${ }^{30-31}$ most strategies rely on a coupling reaction between an acid chloride of a protected cinnamic acid and an appropriately protected derivative of quinic acid. Several reports disclosed the preparation of some cinnamoyl quinic acids or of the corresponding $\gamma$-quinides. ${ }^{32,33,34,35}$ Chemical syntheses for DiCQA derivatives have been nevertheless less reported than those for mono- ${ }^{14,36,37,38,39,40,41}$ or trisubstituted compounds. ${ }^{42,43,44}$ A great variability in the reaction conditions (solvent, catalyst, temperature) can be 
observed. 32,33,42,44,45 Phenol groups were most often protected as carbonates or naturally occurring methoxy, ${ }^{32}$ as acetates (despite some reported difficulties to remove them efficiently), ${ }^{36}$ as methoxymethyl (MOM) ${ }^{36}$ or as $(3,4-$ (methylene-dioxy). ${ }^{43}$ Recently, Jaiswal et al ${ }^{45}$ preferred using an alternative allyl ether protecting group for the successful access to 3-O-epimers of chlorogenic acids derivatives (muco). Activation of the cinnamic acid via carbonyldiimidazole $^{14}$ or as an anhydride was also reported. ${ }^{37,46}$ Tertiary alcohol could also be protected albeit requiring additional steps. ${ }^{32,42}$

Aiming at a most atom-economic and direct approach, we chose to react the key triol 6 with various cinnamoyl chlorides. The synthesis of $\mathbf{6}$ was first optimized to the multi-gram scale from the known intermediate $\mathbf{5}^{47,48,49,50}$ obtained in four steps from D-quinic acid 1 in $40 \%$ overall yield (Scheme 1). Acidic hydrolysis was performed in the presence of a heterogeneous $\mathrm{H}^{+}$resin in $\mathrm{MeOH}$ with an excellent $89 \%$ isolated yield.

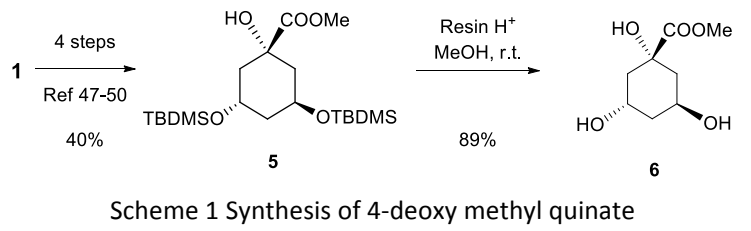

The coupling with the cinnamic counterpart turned out to be surprisingly challenging (Table 1). 6 was first reacted with known diacetylated caffeoylchloride $7 a^{41}$ under reported conditions : DMAP nucleophilic catalysis in pyridine or in DCM/pyridine varying concentration, stoichiometric ratio, temperature and/or reaction time led to very modest yields but above all to unrepeatable results (see Table1, entries 1-3 for selected experiments). Using anhydride $8 \mathbf{a}^{46}$ instead of caffeoyl chloride in pyridine as solvent/base was not more satisfactory even in a shorter reaction time (Entry 4). Analysis of the crude mixtures concluded to partial conversion and only monoesterification of a secondary alcohol more than competitive 1-O-acylation was observed. Rather than adding protection/deprotection steps, we then decided to test DMF/pyridine as another Lewis base-solvent/auxiliary base system ${ }^{51}$ for the reaction of 6 with acid chlorides. ${ }^{52}$ Diesters could eventually be isolated with repetability and reproducibility and fair good yields. ${ }^{53}$ Bis diacetylated dicaffeate 9a was indeed obtained with $76 \%$ yield after $1 \mathrm{~h}$ at $45^{\circ} \mathrm{C}$ using an excess of 7a (entry 5). During our work for optimization, we observed that better results could be obtained for total conversion by addition by portions of acyl chloride instead of increasing the number of molar equivalents leading to difficult purifications. 3 molar equivalents of acid chloride turned out to give the best results. With diallylated acid chloride $\mathbf{7 b}$, the same improvement was observed compared to classical reported conditions albeit with only 59\% yield (Entry 6 vs 7). The same procedure allowed to isolate diferulate 9c (55\%), di- $p$ coumarate $9 \mathrm{~d}(82 \%)$ and di-o-coumarate 9e (62\%) (Entries 8-10). Moreover our good results obtained in the regioselective monoesterification of quinic acid derivatives (unpublished data) led us to repeat some experiments at lower temperature, especially in the classical DCM/Pyridine/DMAP system in which reactions are usually reported at room ${ }^{32,33,38,41}$ or higher temperature. ${ }^{42,44,45}$ Two sets of optimized conditions were assayed (entries 11-12, 13-14, 15-16) giving 9a with excellent 90 and 92\% isolated yields. ${ }^{54}$ Same or lower yield improvement was observed for 9c and 9d (entries 8 vs 13 and 9 vs 15) but an easier purification could be gained under these conditions, lowering the temperature minimizing potential acyl migration to $\mathrm{OH}-1$. Complete conversions were in all cases observed within less than $4 \mathrm{~h}$. 


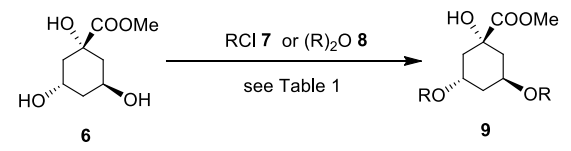

$R=R_{R_{3}}^{R_{1}}$

$$
\begin{aligned}
& \mathrm{aR} \mathrm{R}_{1}=\mathrm{HR}_{2}=\mathrm{OACR} \mathrm{R}_{3}=\mathrm{OAC} \\
& \mathrm{bR} \mathrm{R}_{1}=\mathrm{HR}_{2}=\mathrm{OAll} \mathrm{R}_{3}=\mathrm{OAll} \\
& \mathrm{CR}_{1}=\mathrm{HR}_{2}=\mathrm{OMeR_{3 }}=\mathrm{OAC} \\
& \mathrm{dR} \mathrm{R}_{1}=\mathrm{HR}_{2}=\mathrm{HR}_{3}=\mathrm{OAC} \\
& \mathrm{e} \mathrm{R}_{1}=\mathrm{OACR} \mathrm{R}_{2}=\mathrm{HR}_{3}=\mathrm{H}
\end{aligned}
$$

\begin{tabular}{|c|c|c|c|c|c|c|c|c|}
\hline Entry & $\begin{array}{c}\text { Acylating agent } \\
\text { (molar equiv.) }\end{array}$ & Catalyst & Solvent & $\begin{array}{c}\text { Concentration } \\
\text { (M) }\end{array}$ & Temp. & Time & Product & Yield $^{\mathrm{a}}$ \\
\hline 1 & $7 a(3)$ & DMAP & Pyridine & 0.7 & $\mathrm{rt}$ & 4 days & $9 a$ & $<7 \%$ \\
\hline 2 & $7 a(5)$ & DMAP & Pyridine & 0.05 & $40^{\circ} \mathrm{C}$ & $5 \mathrm{~h}$ & $9 a$ & $n d^{b}$ \\
\hline 3 & $7 a(6)$ & DMAP & DCM/Pyridine & 0.05 & $\mathrm{rt}$ & 7 days & $9 a$ & $40 \%$ \\
\hline 4 & $8 a(3)$ & & Pyridine & 0.02 & $\mathrm{rt}$ & 4 days & $9 a$ & $38 \%$ \\
\hline 5 & $7 a(3)$ & & DMF/Pyridine & 0.4 & $45^{\circ} \mathrm{C}$ & $1 \mathrm{~h}$ & $9 a$ & $76 \%$ \\
\hline 6 & $7 b(5)$ & DMAP & DCM/Pyridine & 0.1 & $\mathrm{rt}$ & $\begin{array}{c}10 \\
\text { days }\end{array}$ & $9 b$ & Trace \\
\hline 7 & $7 b(3)$ & & DMF/Pyridine & 0.4 & $45^{\circ} \mathrm{C}$ & $1 \mathrm{~h} 30$ & $9 b$ & $59 \%$ \\
\hline 8 & $7 c(3)$ & & DMF/Pyridine & 0.4 & $45^{\circ} \mathrm{C}$ & $1 \mathrm{~h}$ & $9 c$ & $55 \%$ \\
\hline 9 & $7 d(3)$ & & DMF/Pyridine & 0.4 & $45^{\circ} \mathrm{C}$ & $1 \mathrm{~h}$ & $9 d$ & $82 \%^{c}$ \\
\hline 10 & $7 e(3)$ & & DMF/Pyridine & 0.4 & $45^{\circ} \mathrm{C}$ & $1 \mathrm{~h}$ & $9 e$ & $62 \%$ \\
\hline 11 & $7 a(3)$ & & DMF/Pyridine & 0.4 & $0^{\circ} \mathrm{C}$ to rt & $4 \mathrm{~h}$ & $9 a$ & $90 \%$ \\
\hline 12 & $7 a(3)$ & DMAP & DCM/Pyridine & 0.1 & $-18^{\circ} \mathrm{C}$ to rt & $4 \mathrm{~h}$ & $9 a$ & $92 \%$ \\
\hline 13 & $7 c(3)$ & & DMF/Pyridine & 0.4 & $0^{\circ} \mathrm{C}$ to $\mathrm{rt}$ & $4 \mathrm{~h}$ & $9 c$ & $69 \%$ \\
\hline 14 & $7 c(3)$ & DMAP & DCM/Pyridine & 0.1 & $-18^{\circ} \mathrm{C}$ to $\mathrm{rt}$ & $4 \mathrm{~h}$ & $9 c$ & $69 \%$ \\
\hline 15 & $7 d(3)$ & & DMF/Pyridine & 0.4 & $0^{\circ} \mathrm{C}$ to rt & $4 \mathrm{~h}$ & $9 d$ & $75 \%$ \\
\hline 16 & $7 d(3)$ & DMAP & DCM/Pyridine & 0.1 & $0^{\circ} \mathrm{C}$ to rt & $4 \mathrm{~h}$ & $9 d$ & $<64 \%^{\mathrm{d}}$ \\
\hline
\end{tabular}

${ }^{a}$ Isolated yield in 3,5-homodiester; ${ }^{b}$ A monoester was detected but not quantified; ${ }^{c}$ Triester was isolated with $7 \%$ yield; ${ }^{d}$ Isolated product was contaminated with polar caffeic acid derivative. 
To get deprotected methyl ester analogs, hydrolysis of 9a under reported acidic conditions $(\mathrm{HCl}$, in $\mathrm{THF}$ or acetone) was again inefficient. The alternative removal of aromatic allyl ethers from $\mathbf{9 b}$ was then performed using $\mathrm{Pd} / \mathrm{C}$ in the presence of $p-\mathrm{TsOH}$ in $\mathrm{MeOH}$ but in a disappointing yield lower than $56 \%$. The hydrolysis of the aromatic acetates was actually successfully performed for 9a with sodium acetate in ethanol/water ${ }^{55}$ in $1 \mathrm{~h}$ at reflux with $61 \%$ isolated yield giving 10a without any hydrolysis of the aliphatic ester groups or any migration observed in the crude product. However, for the other cinnamoyl conjugates, the deprotection turned out to be incomplete under these conditions. With a mild method reported recently, ${ }^{56}$ using hydrazine acetate in DMF at room temperature, all the derivatives could be obtained with very good isolated yields (89-91\%) (Scheme 2 ).

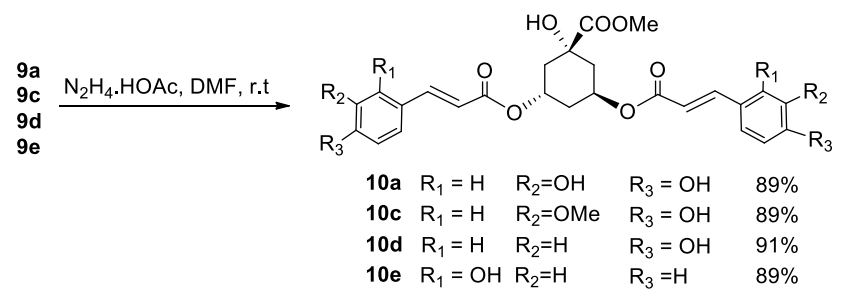

Scheme 2 Deprotection of methyl ester analogs.

Betting for a better solubility of the carboxylic acid derivatives during bioassays and especially for $\mathbf{4 a}$, the main targeted analog of 3, 9a was first reacted with $\mathrm{HCl}$ in THF or acetone to remove both the aromatic acetates and the methyl ester. ${ }^{40}$ But only a complex mixture was obtained after a long reaction time due to very slow conversion. Alternatively, removal of the methyl ester by Lil in EtOAc, $^{57}$ led to the intermediate carboxylic acid 11, which was not isolated and directly submitted to acidic hydrolysis of acetyl groups with $3 \mathrm{M} \mathrm{HCl}$ in acetone under reflux. ${ }^{46,58} 4$ a could be obtained with a very good $87 \%$ overall yield. The same sequence led to $\mathbf{4 c}$ and $\mathbf{4 d}$ with respective $90 \%$ and $93 \%$ yields (Scheme 3 ).

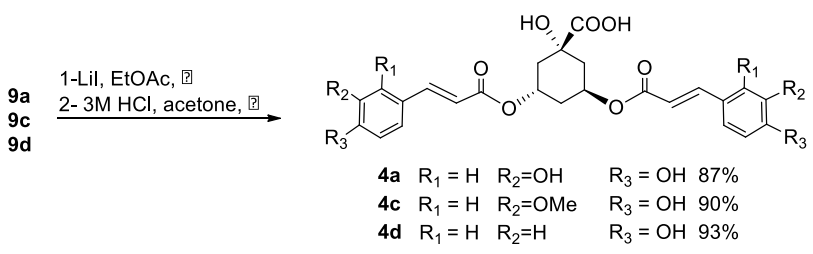

Scheme 3 Synthesis of carboxylic acids analogs

In the course of other ongoing projects around isosorbide, a more rigid bicylic 1,4-diol, dicaffeoyl isosorbide $\mathbf{1 2}$ had also been prepared. ${ }^{59} 12$ was included in bioassays with the seven compounds 10a, 10c, 10d, 10e, 4a, 4c, 4d for comparison (Figure 2). All the intermediates were fully characterized. Higher order quantum effects are observed through the highly complex splitting pattern of signals in ${ }^{1} \mathrm{H}$ NMR spectra. The interesting pseudo-symmetry of these compounds make difficult to distinguish $\mathrm{H}-3$ and $\mathrm{H}-5$ signals but one $\mathrm{H}-4$ signal is well-resolved in protected compounds $\mathbf{9}$ : the predominance of rapidly interconverted chair-like conformers is thus supported by a mean value of ${ }^{3} \mathrm{~J}$ coupling constant of $9-10 \mathrm{~Hz}$ between $\mathrm{H}-4$ axial and $\mathrm{H}-3 / \mathrm{H}-5$. Preparative HPLC before bioassays confirmed the high purity of the different samples. ${ }^{1} \mathrm{H} N M R\left(\mathrm{CD}_{3} \mathrm{OD}\right)$ of $\mathbf{4 a}$ and $\mathbf{4 d}$ at different time intervals confirmed the stability of this compound at least over $60 \mathrm{~h}$ compared to $\mathbf{3}$ (see Supplementary information). ${ }^{28}$

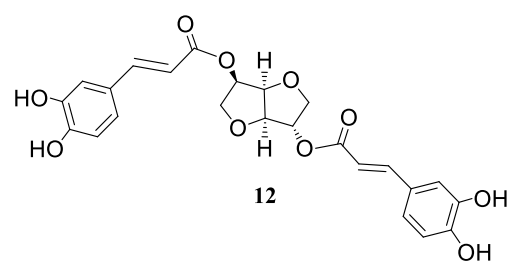

Figure 2 : Dicaffeoyl isosorbide 12

\section{Insecticidal bioassays}

10a, 10c, 10d, 10e, 4a, 4c, 4d and 12 were incorporated in culture media during nymphal development of the pea aphid Acyrthosiphon pisum. The natural compound $\mathbf{3}$ was used as control. This specialist and model aphid species (A. pisum) was indeed shown to be highly sensitive to $3{ }^{27}$ and was therefore selected for optimal suitability for growth-inhibition/ toxicity bioassays.

Bioassays were scored for mortality data, summarized as median lethal time $50 \%$ (LT50) resulting from a survival analysis (i.e. the median time necessary for killing $50 \%$ of the insect population). All concentrations were analysed separately in a parametric survival analysis with a log-normal fit, taking the "compound" as a factor (nominal, 9 levels). Dose $500 \mu \mathrm{M}$ gave the most discriminating analysis (likelihood ratio test with a $\chi 2$ of 200.6, $p=4.810^{-39}$ ), and we will only discuss this dose level, while growth-rate analyses will show all dose effects (Table 2). Table S3 in supplementary information displays all eight survival curves at dose $500 \mu \mathrm{M}$. Toxicity (mortality) features of the most active compounds 10a, 10e, 4a, 4c, 4d are summarized in Figure 3 for a $500 \mu \mathrm{M}$ dose according to decreasing activities from $\mathbf{4 a}$ to $\mathbf{1 0 a}$. 


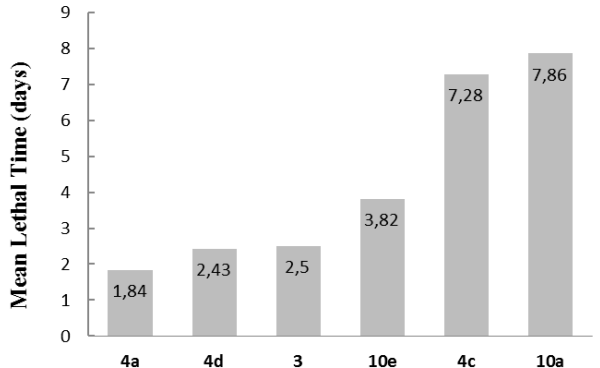

Figure 3 Survival analysis. LT50 mean values per active compound

Growth inhibition was measured by weighing and was analysed by a set of two one-way Anova (Table 2), comparing either the dose effects per compound (capital lettering) or the compound effect, dose per dose (small lettering). As five independent experiments were needed to score all compounds, a standard normalisation of data by the results of the control condition (diet alone) was performed. Using this normalisation for weights resulted in a quite reproducible scoring of growth inhibition for the control natural molecule $\mathbf{3}$ used for every experimental batch: a two way Anova for dose/experiment, with interactions, resulted in a very significant "dose" effect $(p<0.001)$, a non-significant "experiment" effect $(p=0.61)$ and a non-significant interaction $(p=0.24)$. The effect of 3 was therefore represented in Table 2 by the global results of the 5 batches used for this molecule.

These growth inhibition analyses mainly underline the activity of two new molecules, superior or close to the one of the natural product $\mathbf{3}$, namely $\mathbf{4 a}$ and $\mathbf{4 d}$. $\mathbf{1 0 d}$ showed no inhibitory effect on the growth of the pea aphid, while $\mathbf{1 2}$ displays a slight stimulatory effect at the highest $1 \mathrm{mM}$ dose. As a result from all the "compound" factor ANOVAs (dose by dose), that read vertically in Table 2, one might rank the global toxicity index of compounds as: $4 \mathrm{a}>4 \mathrm{~d}>3 \approx 10 \mathrm{e}>4 \mathrm{c} \geq 10 \mathrm{a}>10 \mathrm{~d} \approx 10 \mathrm{c}>12$.

Table 2 : Growth inhibition assay over nymphal development (7 days) of the pea aphid Acyrthosiphon pisum. Results are shown as means (\% of control growth) \pm SE, with ANOVA and mean comparisons (Tukey-Kramer HSD test) over lines (dose effect, capital lettering, $\rightarrow$ ) and over columns (molecule effect, small lettering, $\boldsymbol{\downarrow}$ )

\begin{tabular}{|c|c|c|c|c|c|c|c|c|}
\hline \multirow{2}{*}{ Compound } & \multicolumn{2}{|l|}{ control } & \multicolumn{2}{|c|}{$\begin{array}{l}\text { Dose } \\
(\mathrm{mM})\end{array}$} & \multicolumn{3}{|c|}{ Anova } & \multirow[t]{2}{*}{ Experiment $^{\mathrm{iv}}$} \\
\hline & 0 & 0.125 & 0.25 & 0.5 & 1 & F-ratio & $p$ & \\
\hline \multirow[t]{2}{*}{$3^{i}$} & $1.00 \pm 0.04$ & $0.98 \pm 0.03$ & $0.70 \pm 0.03$ & $0.36 \pm 0.04$ & $0^{i i}$ & 35.3 & $1.410^{-18}$ & $1-5$ \\
\hline & $\rightarrow \mathrm{A}, \mathrm{a} \downarrow$ & $A, b$ & $\mathrm{~B}, \mathrm{bc}$ & $\mathrm{C}, \mathrm{d}$ & - & $(3 d f)$ & & \\
\hline $4 a$ & $1.00 \pm 0.03$ & $0^{\mathrm{ii}}$ & $0^{\mathrm{ii}}$ & $0^{i i}$ & $0^{\mathrm{ii}}$ & na ${ }^{\mathrm{iii}}$ & naii & 2 \\
\hline \multirow[t]{2}{*}{$10 a$} & $1.00 \pm 0.08$ & $1.07 \pm 0.05$ & $1.03 \pm 0.06$ & $0.96 \pm 0.10$ & $0.53 \pm 0.04$ & 6.8 & $6.510^{-5}$ & 3 \\
\hline & $\mathrm{A}, \mathrm{a}$ & $A, a b$ & $\mathrm{~A}, \mathrm{a}$ & $\mathrm{A}, \mathrm{a}$ & $B, C$ & (4 df) & & \\
\hline \multirow[t]{2}{*}{$10 \mathrm{e}$} & $1.00 \pm 0.08$ & $1.29 \pm 0.09$ & $0.65 \pm 0.08$ & $0.38 \pm 0.05$ & $0^{\mathrm{ii}}$ & 21.3 & $2.010^{-10}$ & 3 \\
\hline & $\mathrm{B}, \mathrm{a}$ & $\mathrm{A}, \mathrm{a}$ & $\mathrm{C}, \mathrm{C}$ & $\mathrm{C}, \mathrm{d}$ & & (3 df) & & \\
\hline \multirow[t]{2}{*}{$4 d$} & $1.00 \pm 0.04$ & $0.93 \pm 0.16$ & $0.77 \pm 0.21$ & $0^{\mathrm{ii}}$ & $0^{\mathrm{ii}}$ & 4.3 & $2.510^{-2}$ & 4 \\
\hline & $\mathrm{A}, \mathrm{a}$ & $A B, a b$ & $\mathrm{~B}, \mathrm{abc}$ & & & $(2 \mathrm{df})$ & & \\
\hline \multirow[t]{2}{*}{$10 d$} & $1.00 \pm 0.04$ & $0.98 \pm 0.04$ & $1.00 \pm 0.05$ & $0.91 \pm 0.04$ & $0.89 \pm 0.04$ & 1.28 & 0.28 (ns) & 4 \\
\hline & $\mathrm{A}, \mathrm{a}$ & $A, b$ & $\mathrm{~A}, \mathrm{a}$ & $A, a b$ & $A, b$ & $(4 d f)$ & & \\
\hline \multirow[t]{2}{*}{$4 c$} & $1.00 \pm 0.06$ & $1.01 \pm 0.07$ & $0.90 \pm 0.02$ & $0.67 \pm 0.05$ & $0.57 \pm 0.08$ & 10.4 & $8.310^{-7}$ & 5 \\
\hline & $A, a$ & $A, b$ & $A, a b$ & B,bcd & $\mathrm{B}, \mathrm{bc}$ & (4 df) & & \\
\hline \multirow[t]{2}{*}{$10 c$} & $1.00 \pm 0.06$ & $1.03 \pm 0.04$ & $0.86 \pm 0.05$ & $0.87 \pm 0.05$ & $0.75 \pm 0.06$ & 3.9 & $6.210^{-3}$ & 5 \\
\hline & $\mathrm{A}, \mathrm{a}$ & $A B, a b$ & $A B, a b c$ & $A B, a b c$ & $\mathrm{~B}, \mathrm{bc}$ & (4 df) & & \\
\hline \multirow[t]{2}{*}{12} & $1.00 \pm 0.06$ & $0.92 \pm 0.05$ & $0.94 \pm 0.06$ & $0.79 \pm 0.05$ & $1.22 \pm 0.10$ & 5.4 & $4.610^{-4}$ & 1 \\
\hline & $A B, a$ & $B, b$ & $B, a b$ & $\mathrm{~B}, \mathrm{abc}$ & $\mathrm{A}, \mathrm{a}$ & $(4 \mathrm{df})$ & & \\
\hline Anova & $n a^{\mathrm{iii}}$ & 4.4 & 5.6 & 7.8 & 19.9 & & & \\
\hline \multicolumn{9}{|l|}{ F-ratio } \\
\hline \multirow[t]{2}{*}{$\mathrm{p}$} & $n a^{i i i}$ & $1.410^{-4}$ & $6.210^{-6}$ & $3.810^{-7}$ & $3.510^{-13}$ & & & \\
\hline & & (7 df) & (7 df) & (6 df) & (5 df) & & & \\
\hline
\end{tabular}

${ }^{i}: \mathbf{3}$ was the repeated control condition for all experiments, presented data are that of all grouped experiments. See text for inter-experiment statistics.

${ }^{\mathrm{ii}}: 0$ is no survivor at day 7

iii:not applicable

${ }^{\text {iv }}$ :Five independent experiments were used (see text), and control data were checked for consistency (ANOVA ns on dose 0), and pooled after normalization 


\section{Experimental}

\section{General}

Reagents and solvents were supplied by Aldrich, Acros, Lancaster, Alfa Aesar, Fluka or $\mathrm{TCl}$ and purchased at the highest commercial quality to be used without further purification. NMR spectra were recorded on a Bruker $300\left({ }^{1} \mathrm{H}: 300 \mathrm{MHz} ;{ }^{13} \mathrm{C}: 75 \mathrm{MHz}\right)$, Bruker 400 $\left({ }^{1} \mathrm{H}: 400 \mathrm{MHz} ;{ }^{13} \mathrm{C}: 100 \mathrm{MHz}\right)$, or Bruker $500\left({ }^{1} \mathrm{H}: 500 \mathrm{MHz} ;{ }^{13} \mathrm{C}: 125\right.$ $\mathrm{MHz}$ ) spectrometers, at $295-298 \mathrm{~K}$, using $\mathrm{CDCl}_{3}, \mathrm{CD}_{3} \mathrm{OD}$ or $\mathrm{D}_{2} \mathrm{O}$ as solvents. The chemical shifts ( $\delta \mathrm{ppm})$ are referenced to the solvent residual peak ${ }^{60}$ and coupling constants $(\mathrm{Hz})$ are reported in the standard fashion. The following abbreviations are used to explain the multiplicities: $\mathrm{s}=$ singlet, $\mathrm{d}=$ doublet, $\mathrm{t}=$ triplet, $\mathrm{q}=$ quartet, quint. = quintuplet, sext. = sextuplet, hept. = heptuplet, $\mathrm{m}=$ multiplet, $b r=$ broad. Electrospray ionization (ESI) mass spectrometry (MS) experiments were performed on a Thermo Finnigan LCQ Advantage mass. High-resolution mass spectra (HRMS) were recorded on a Finnigan Mat 95xL mass spectrometer using electrospray. Analytical thin-layer chromatography was carried out on silica gel Merck 60 D254 (0.25 mm). Flash chromatography was performed on Merck Si 60 silica gel (40-63 $\mu \mathrm{m})$. Infra-red (IR) spectra were recorded with a IRAffinity-1 Shimadzu spectrometer using Attenuated Total Reflectance (ATRMiracle), and the wavenumbers are expressed in $\mathrm{cm}^{-1}$. Optical rotations were measured on a Perkin Elmer 241 or Jasco P1010 polarimeter with a $10 \mathrm{~cm}$ cell (concentration c expressed as g/100 $\mathrm{mL}$ ). Melting points were measured using Büchi apparatus B-540. All data for chlorogenic acids or related derivatives presented in this paper use the recommended IUPAC numbering system. The assignment of NMR signals has been made according to the numbering shown in the general structures below (Figure 4). ${ }^{1}$
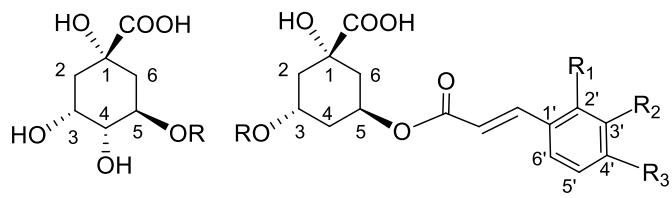

Figure 4 Numbering for CQA and deoxy analogs

\section{Material and methods for bioassays}

The natural compound 3 (3,5-dicaffeoyl quinic acid), was extracted with ethanol:water (70:30) from lyophilized non-tuberized roots of sweet potato Ipomea batatas and purified by semi-preparative HPLC on a Hibar $^{\circledast} 250 \mathrm{~mm} \times 25 \mathrm{~mm}$ column filled with Lichrospher ${ }^{\circledR}$ $100 \mathrm{RP} 18 \mathrm{e} 5 \mu \mathrm{M}$ stationary phase. The compound was eluted with an acidified water/methanol gradient. ${ }^{27}$

Compounds 10a, 10c, 10d, 10e, 4a, 4c, 4d and 12 were administrated pure by weighing after HPLC purification, solubilized at $1 \mathrm{mM}$ in a standard AP3 artificial diet for the pea aphid Acyrthosiphon pisum, ${ }^{61}$ and diluted 3 times to obtain the experimental dose range (125 $\mu \mathrm{M}-1 \mathrm{mM})$. Stability of compounds was checked by HPLC analysis in AP3 artificial diet samples after feeding bioassays. Toxicity was evaluated by scoring survival daily over the whole nymphal life of the pea aphid (7 days), starting from neonate aphids born on faba bean leaves. Growth was also measured by weighing adult aphids on an analytical microbalance (Mettler AE163) at the closest $10 \mu \mathrm{g}$, as fully described previously for testing peptide toxicity. ${ }^{62}$ Aphid weights were analysed by ANOVA followed by Tukey-Kramer HSD test for comparing multiple means (treating separately either the dose factor for every compound, or the compound factor, at each dose; see Table 2). All analyses were made with JMP software, v11 (SAS Institute Cary USA, MacOS version).

Five separate experiments were needed to test all eight compounds (two-weeks apart each from the other), and the original compound 3 was used/repeated in each of the five tests. An ANOVA was performed on all dose 0 data and confirmed global interexperiment consistency. All data (normalized on the respective dose 0 mean) from the 5 experiments were then used for the control compound $\mathbf{3}$ in the statistical analysis.

\section{Chemistry}

Methyl (3S,5S)-1,3,5-trihydroxycyclohexanecarboxylate or methyl 4-deoxy quinate 6. The disilylated compound 5 (106.7 $\mathrm{mg}, 0.25$ $\mathrm{mmol}$ ) was stirred in $\mathrm{MeOH}(2 \mathrm{~mL})$ with Amberlyst $\mathrm{A} 15\left(\mathrm{H}^{+}\right.$form $)$at room temperature for $24 \mathrm{~h}$. After filtration and evaporation, the crude product was purified by flash chromatography (pentane/ethyl acetate 50/50 then ethyl acetate/methanol 90/10) to provide 6 (colorless oil then white solid on standing, $42.7 \mathrm{mg}, 89 \%$ ). $[\alpha]_{D}^{25}=-$ 3.7 (c 1.06, $\mathrm{CH}_{3} \mathrm{OH}$ ); IR (ATR) 3350, 2951, 2930, 2855, 1173, 1730, $1661,1435,1371,1281,1240,1134,1057,1016 \mathrm{~cm}^{-1} ;{ }^{1} \mathrm{H}$ NMR (400 $\left.\mathrm{MHz}, 296 \mathrm{~K}, \mathrm{CD}_{3} \mathrm{OD}\right) \delta(\mathrm{ppm})=4.24-4.16\left(\mathrm{~m}, 2 \mathrm{H}, \mathrm{H}_{3}\right.$ and $\left.\mathrm{H}_{5}\right), 3.73(\mathrm{~s}$, $3 \mathrm{H}, \mathrm{OMe}$ ), $2.08\left(\mathrm{dd}, 1 \mathrm{H},{ }^{2} J 13.8 \mathrm{~Hz},{ }^{3} \mathrm{~J}_{\mathrm{H}_{5} / \mathrm{H}_{6}} 3.3 \mathrm{~Hz}, \mathrm{H}_{6}\right.$ or $\left.\mathrm{H}_{2}\right), 2.00(\mathrm{~m}$, $2 \mathrm{H}, \mathrm{H}_{2}$ or $\mathrm{H}_{6}$ and $\left.\mathrm{H}_{4}\right), 1.88$ (dd, $1 \mathrm{H},{ }^{2} J 12.8 \mathrm{~Hz},{ }^{3} \mathrm{H}_{\mathrm{H}_{2} / \mathrm{H}_{3}} 9.1 \mathrm{~Hz}, \mathrm{H}_{2}$ or $\mathrm{H}_{6}$ ), 1.77 (dd, $1 \mathrm{H},{ }^{2} \mathrm{~J} 13.8 \mathrm{~Hz},{ }^{3} \mathrm{~J} 4.8 \mathrm{~Hz}, \mathrm{H}_{6}$ or $\mathrm{H}_{2}$ ), 1.60 (ddd, $1 \mathrm{H},{ }^{2}$ J $12.7 \mathrm{~Hz},{ }^{3} J_{\mathrm{H}_{4 a}} / \mathrm{H}_{3 \mathrm{a}}$ ou $5 \mathrm{a} 9.2 \mathrm{~Hz},{ }^{3} \mathrm{~J}_{\mathrm{H}_{4}} / \mathrm{H}_{3 \mathrm{e}}$ ou $\left.\mathrm{H5e} 3.0 \mathrm{~Hz}, \mathrm{H}_{4 \mathrm{a}}\right) ;{ }^{13} \mathrm{C} \mathrm{NMR}$ $\left(\mathrm{CD}_{3} \mathrm{OD}, 295 \mathrm{~K}, 100 \mathrm{MHz}\right) \delta$ (ppm) 176.1 (COOMe), $76.8\left(\mathrm{C}_{1}\right), 67.8\left(\mathrm{C}_{5}\right.$ or $\left.\mathrm{C}_{3}\right), 64.0\left(\mathrm{C}_{3}\right.$ or $\left.\mathrm{C}_{5}\right), 52.8(\mathrm{OMe}), 43.8\left(\mathrm{C}_{2}\right.$ or $\left.\mathrm{C}_{6}\right), 42.0\left(\mathrm{C}_{4}\right), 40.3\left(\mathrm{C}_{6}\right.$ or $\mathrm{C}_{2}$ ). MS-ESI m/z [M+Na] $]^{+}$213.1; HRMS-ESI m/z [M+Na] $]^{+}$calcd for $\mathrm{C}_{8} \mathrm{H}_{14} \mathrm{NaO}_{5}$ 213.0733; found 213.0733.

General procedures for the synthesis of diesters 9 of deoxy methyl quinate.

Procedure A : To a solution of triol 6 in DMF / pyridine ( $v / v 3 / 2, c=$ $0.4 \mathrm{M})$ under nitrogen was added the protected cinnamoyl chloride ( 3 equiv.) The mixture was stirred at $45^{\circ} \mathrm{C}$ until completion, in general after $1 \mathrm{~h}$. Methanol was added and after $15 \mathrm{~min}$ under stirring, the resulting mixture was concentrated, diluted with EtOAc, washed with $1 \mathrm{M} \mathrm{HCl}$ then with a saturated $\mathrm{NaHCO}_{3}$ solution then with brine. The organic layer was dried over $\mathrm{Na}_{2} \mathrm{SO}_{4}$, concentrated and purified by flash chromatography on silica using the appropriate eluent.

Procedure B : Similar to procedure A except that the mixture was stirred at $0^{\circ} \mathrm{C}$ until completion, in general after $4 \mathrm{~h}$.

Procedure $\mathrm{C}$ : To a solution of triol $\mathbf{6}$ in dichloromethane / pyridine ( $v / v 5 / 1, c=0.1 \mathrm{M})$ under nitrogen was added the protected cinnamoyl chloride ( 3 equiv.) and DMAP ( $20 \mathrm{~mol} \%)$. The mixture was stirred at $-18^{\circ} \mathrm{C}$ to room temperature until completion, in general after $4 \mathrm{~h}$. Work-up as in procedures $\mathrm{A}$ and $\mathrm{B}$.

Methyl (3S,5S) 1-hydroxy-3,5 bis[[(2E)-3-(3,4-diacetoxyphenyl)-1- 
oxo-2-propen-1-yl]oxy]cyclohexanecarboxylate 9a. Synthesized according to the general procedure B using diacetylated caffeoyl chloride $^{41}$ and methyl 4-deoxy quinate 6 (105 mg, $0.553 \mathrm{mmol}$ ). 9a was obtained as a white solid ( $348 \mathrm{mg}$, yield: $92 \%$ ) after purification by flash chromatography on silica using $\mathrm{DCM} / \mathrm{Et}_{2} \mathrm{O} 12 / 1$ to $6 / 1$. $[\alpha]_{D}^{25}=-46.9$ (c $\left.1.215, \mathrm{CH}_{2} \mathrm{Cl}_{2}\right) ;$ m.p. $=93-94^{\circ} \mathrm{C}\left(\mathrm{CH}_{2} \mathrm{Cl}\right.$ ) ; IR(ATR) 2943, 2832, 1775, 1742, 1713, 1639, 1504, 1449, 1427, 1371, 1321, $1242,1205,1180,1147,1132,1111,1022 \mathrm{~cm}^{-1} ;{ }^{1} \mathrm{H}$ NMR $\left(\mathrm{CDCl}_{3}\right.$, $298 \mathrm{~K}, 500 \mathrm{MHz}) \delta(\mathrm{ppm})=7.63$ and $7.58\left(2 \mathrm{~d}, 2 \mathrm{H},{ }^{3} \mathrm{~J} 15.9 \mathrm{~Hz}\right.$, $\mathrm{CH}=\mathrm{CHCO}), 7.39$ and $7.38\left(2 \mathrm{dd}, 2 \mathrm{H}, J_{\text {ortho }} 8.4 \mathrm{~Hz}, J_{\text {meta }} 1.9 \mathrm{~Hz}, 2 \mathrm{xH}_{6^{\prime}}\right)$, 7.35 and $7.34\left(2 \mathrm{~d}, 2 \mathrm{H}, J_{\text {meta }} 1.9 \mathrm{~Hz}, 2 \mathrm{x} \mathrm{H}_{2^{\prime}}\right), 7.20\left(\mathrm{~d}, 2 \mathrm{H}, J_{\text {ortho }} 8.4 \mathrm{~Hz}\right.$, 2x $\left.\mathrm{H}_{5^{\prime}}\right), 6.40$ and $6.32\left(2 \mathrm{~d}, 2 \mathrm{H},{ }^{3} \mathrm{~J} 15.9 \mathrm{~Hz}, \mathrm{CH}=\mathrm{CHCO}\right.$ ), 5.51 (ddd, $1 \mathrm{H}$, IJ $13.8 \mathrm{~Hz},{ }^{3}$ J $9.8 \mathrm{~Hz},{ }^{3}$ J $3.9 \mathrm{~Hz}, \mathrm{H}_{3 \text { ax ou } 5 a x}$ ), 5.46 (ddd, $1 \mathrm{H},{ }^{3} \mathrm{~J}={ }^{3} \mathrm{~J} 7.8 \mathrm{~Hz}$, 3 $\left.3.9 \mathrm{~Hz}, \mathrm{H}_{\text {3eq ou } 5 \text { eq }}\right), 3.76\left(\mathrm{~s}, 1 \mathrm{H}, \mathrm{OH}_{1}\right), 3.74(\mathrm{~s}, 3 \mathrm{H}, \mathrm{COOMe}), 2.28$, 2.271 and $2.268\left(3 \mathrm{~s}, 12 \mathrm{H}, \mathrm{OCOCH}_{3}\right), 2.32\left(\mathrm{~m}, 1 \mathrm{H}, \mathrm{H}_{4}\right), 2.23-2.18(\mathrm{~m}$, $2 \mathrm{H}, \mathrm{H}_{2}$ and $\left.\mathrm{H}_{6}\right), 2.08-2.00\left(\mathrm{~m}, 2 \mathrm{H}, \mathrm{H}_{2}\right.$ and $\left.\mathrm{H}_{6}\right), 1.87$ (ddd, $1 \mathrm{H},{ }^{2} \mathrm{~J} 13.6$ $\left.\mathrm{Hz},{ }^{3} \mathrm{~J} 10.3 \mathrm{~Hz},{ }^{3} \mathrm{~J} 3.45 \mathrm{~Hz}, \mathrm{H}_{4 \mathrm{a}}\right) ;{ }^{13} \mathrm{C} \mathrm{NMR}\left(\mathrm{CDCl}_{3}, 298 \mathrm{~K}, 125 \mathrm{MHz}\right) \delta$ $(\mathrm{ppm})=175.0,168.1(2)$ and $168.0(2)\left(4 \mathrm{xOCOCH}_{3}\right), 165.8$ and 165.5 (2xCOO), 143.59 and $143.57\left(\mathrm{C}_{\mathrm{q}}\right), 143.3$ and $143.2(\underline{\mathrm{CH}}=\mathrm{CHCO})$, 142.5 and $142.4\left(\mathrm{C}_{\mathrm{q}}\right), 133.2$ and $133.1\left(\mathrm{C}_{\mathrm{q}}\right), 126.5$ and $126.4\left(\mathrm{C}_{6^{\prime}}\right)$, 124.0 and $123.9\left(C_{5^{\prime}}\right), 122.9$ and $122.8\left(C_{2^{\prime}}\right), 119.4$ and 119.2 $(\mathrm{CH}=\underline{\mathrm{CHCO}}), 74.7\left(\mathrm{C}_{1}\right), 68.9\left(\mathrm{C}_{3}\right.$ ou $\left.\mathrm{C}_{5}\right), 66.9\left(\mathrm{C}_{5}\right.$ ou $\left.\mathrm{C}_{3}\right), 53.1$ (OMe), $39.7\left(C_{2}\right.$ ou $\left.C_{6}\right), 36.7\left(C_{2}\right.$ ou $\left.C_{6}\right), 35.0\left(C_{4}\right), 20.7(2), 20.62$ and 20.60 $\left(4 \mathrm{x} \quad \mathrm{OCOCH}_{3}\right) ;$ HRMS-ESI m/z $[\mathrm{M}+\mathrm{Na}]^{+}$calcd for $\mathrm{C}_{34} \mathrm{H}_{34} \mathrm{NaO}_{15}$ 705.1790; found 705.1780.

Methyl (3S,5S) 1-hydroxy-3,5 bis[[(2E)-3-(3,4-di-( prop-2-en-1yloxy)phenyl)-1-oxo-2-propen-1-yl]oxy]cyclohexanecarboxylate 9b. Synthesized according to the general procedure $\mathbf{A}$ using diallylated caffeoyl chloride ${ }^{45}$ and 6 (102 $\left.\mathrm{mg}, 0.537 \mathrm{mmol}\right)$. Purification by flash chromatography on silica using petroleum ether/EtOAc 50/50, provided the triester $(51 \mathrm{mg}, 10 \%)$ and diester 9b (214 mg, 59\%) as white solids. $[\alpha]_{D}^{25}=-140.8\left(\mathrm{c} 0.86, \mathrm{CH}_{2} \mathrm{Cl}_{2}\right.$ ); m.p. $89-91^{\circ} \mathrm{C}$; IR(ATR) 2922, 2827, 1706, 1631, 1597, 1510, 1456, $1423,1362,1335,1304,1246,1223,1165,1134,1090,1067 \mathrm{~cm}^{-1}$; ${ }^{1} \mathrm{H} \mathrm{NMR}\left(\mathrm{CDCl}_{3}, 298 \mathrm{~K}, 300 \mathrm{MHz}\right) \delta(\mathrm{ppm})=7.63$ and $7.58\left(2 \mathrm{~d}, 2 \mathrm{H},{ }^{3} \mathrm{~J}\right.$ $15.9 \mathrm{~Hz}, \mathrm{CH}=\mathrm{CHCO}), 7.08\left(\mathrm{~m}, 4 \mathrm{H}, \mathrm{CH}_{\mathrm{Ar}}\right), 6.87\left(\mathrm{~m}, 2 \mathrm{H}, \mathrm{CH}_{\mathrm{Ar}}\right), 6.31$ and $6.23\left(2 \mathrm{~d}, 2 \mathrm{H},{ }^{3} \mathrm{~J} 15.9 \mathrm{~Hz}, \mathrm{CH}=\mathrm{CHCO}\right), 6.07\left(\mathrm{~m}, 4 \mathrm{H}, \mathrm{CH}_{2}=\mathrm{CHCH}_{2} \mathrm{O}\right), 5.60-$ $5.50\left(\mathrm{~m}, 2 \mathrm{H}, \mathrm{H}_{3}\right.$ and $\left.\mathrm{H}_{5}\right), 5.42-5.39\left(\mathrm{~m}, 2 \mathrm{H}, \mathrm{CH}_{2}=\mathrm{CHCH}_{2} \mathrm{O}\right), 5.30(\mathrm{~m}$, $\left.4 \mathrm{H}, \mathrm{CH}_{2}=\mathrm{CHCH}_{2} \mathrm{O}\right), 4.64\left(\mathrm{~m}, 8 \mathrm{H}, \mathrm{CH}_{2}=\mathrm{CHCH}_{2} \mathrm{O}\right), 2.32\left(\mathrm{~m}, 1 \mathrm{H}, \mathrm{H}_{2}\right.$ or $\mathrm{H}_{4}$ or $\left.\mathrm{H}_{6}\right), 2.24-2.02\left(\mathrm{~m}, 4 \mathrm{H}, \mathrm{H}_{2}\right.$ or $\mathrm{H}_{4}$ or $\left.\mathrm{H}_{6}\right), 1.86$ (ddd, $1 \mathrm{H}, J 13.7 \mathrm{~Hz}, J$ $10.4 \mathrm{~Hz}, J 3.4 \mathrm{~Hz}, \mathrm{H}_{2}$ or $\mathrm{H}_{4}$ or $\left.\mathrm{H}_{6}\right) ;{ }^{13} \mathrm{CNMR}\left(\mathrm{CDCl}_{3}, 296 \mathrm{~K}, 75 \mathrm{MHz}\right) \delta$ $(\mathrm{ppm})=175.1$ (COOMe), 166.5 and $166.3(\mathrm{OCO}), 150.8\left(2 \times \mathrm{C}_{\mathrm{q}}\right), 148.7$ $\left(2 \times \mathrm{C}_{\mathrm{q}}\right), 145.4$ and $145.1(\underline{\mathrm{CH}}=\mathrm{CHCO}), 133.2133 .1,133.05$ and 133.01 $\left(4 \times \mathrm{CH}_{2}=\mathrm{CHCH}_{2} \mathrm{O}\right), 127.64,127.57(2 \times \mathrm{Cq}), 123.2$ and 122.9 (2xCHAr), $118.15,118.11,118.1,118.07\left(4 \times \mathrm{CH}_{2}=\mathrm{CHCH}_{2} \mathrm{O}\right), 115.88$ and 115.84 $(\mathrm{CH}=\mathrm{CHCO}), 113.50,113.44,112.7$ and $112.5\left(4 \times \mathrm{CH}_{\mathrm{Ar}}\right), 75.0\left(\mathrm{C}_{1}\right)$, $70.1,70.0$, and $69.8(2)\left(4 \times \mathrm{CH}_{2}=\mathrm{CHCH}_{2} \mathrm{O}\right), 68.8$ and $66.6\left(\mathrm{C}_{3}\right.$ and $\left.\mathrm{C}_{5}\right)$, 53.2(OMe), 40.0, 36.9 and $35.2\left(\mathrm{C}_{2}, \mathrm{C}_{4}\right.$ and $\left.\mathrm{C}_{6}\right)$; HRMS-ESI m/z $[\mathrm{M}+\mathrm{Na}]^{+}$calcd for $\mathrm{C}_{38} \mathrm{H}_{42} \mathrm{NaO}_{11}$ 697.2619; found 697.2615.

Methyl (3S,5S) 1-hydroxy-3,5 bis[[(2E)-3-(3-acetoxy,4methoxyphenyl)-1-oxo-2-propen-1-yl]oxy]cyclohexanecarboxylate 9c. Synthesized according to the general procedure $\mathbf{C}$ using acetylated feruloyl chloride and methyl 4-deoxy quinate $6(117 \mathrm{mg}$, $0.616 \mathrm{mmol}$ ). $9 \mathrm{c}$ was obtained as a white solid (266 mg, yield: $69 \%$ ) after purification by flash chromatography on silica using DCM/Et ${ }_{2} \mathrm{O}$ 10/1. $[\alpha]_{D}^{25}=-74.5$ (c $1.05, \mathrm{CH}_{2} \mathrm{Cl}_{2}$ ); m.p. $90-92^{\circ} \mathrm{C}$; IR(ATR) 1765, $1709,1638,1508,1464,1418,1369,1298,1263,1215,1198,1153$, $1123,1033,1011 \mathrm{~cm}^{-1} ;{ }^{1} \mathrm{H}$ NMR $\left(\mathrm{CDCl}_{3}, 296 \mathrm{~K}, 300 \mathrm{MHz}\right) \delta(\mathrm{ppm})=$ 7.65 and $7.60\left(2 \mathrm{~d}, 2 \mathrm{H},{ }^{3} \mathrm{~J} 15.9 \mathrm{~Hz}, \mathrm{CH}=\mathrm{CHCO}\right), 7.12-7.02(\mathrm{~m}, 6 \mathrm{H}$,
$\left.\mathrm{CH}_{\mathrm{Ar}}\right), 6.41$ and $6.33\left(2 \mathrm{~d}, 2 \mathrm{H},{ }^{3} \mathrm{~J} 15.9 \mathrm{~Hz}, \mathrm{CH}=\mathrm{CHCO}\right), 5.55$ and 5.49 $\left(2 \mathrm{~m},{ }^{3} \mathrm{~J} 9.3 \mathrm{~Hz},{ }^{3} \mathrm{~J} 3.3 \mathrm{~Hz}, \mathrm{H}_{5}\right.$ and $\left.\mathrm{H}_{3}\right), 3.85$ and $3.84(2 \mathrm{~s}, 2 \times 3 \mathrm{H}$, $2 \times O M e), 3.76(\mathrm{~s}, 3 \mathrm{H}, \mathrm{COOMe}), 2.30(\mathrm{~s}, 6 \mathrm{H}, 2 \times O A c), 2.28\left(\mathrm{~m}, 1 \mathrm{H}, \mathrm{H}_{4 \mathrm{e}}\right)$, 2.22-2.18 and 2.11-2.04(m, $2 \times 2 \mathrm{H}, 2 \mathrm{xH}_{6}$ and $\left.2 \mathrm{xH}_{2}\right), 1.88\left(\mathrm{ddd}, 1 \mathrm{H},{ }^{2} \mathrm{~J}\right.$ $13.6 \mathrm{~Hz},{ }^{3} \mathrm{~J} 10.4 \mathrm{~Hz},{ }^{3}$ J $\left.3.4 \mathrm{~Hz}, \mathrm{H}_{4}\right) ;{ }^{13} \mathrm{C} \mathrm{NMR}\left(\mathrm{CDCl}_{3}, 295 \mathrm{~K}, 100 \mathrm{MHz}\right) \delta$ $(\mathrm{ppm})=175.0(\mathrm{COOMe}), 168.8\left(2 \times \mathrm{xOCCH}_{3}\right), 166.1$ and $165.8(\mathrm{COO})$, $151.4\left(2 \times \mathrm{C}_{\mathrm{q}}\right), 144.6$ and $144.4(\mathrm{CH}=\mathrm{CHCO}), 141.54$ and $141.51\left(\mathrm{C}_{\mathrm{q}}\right)$, 133.34 and $133.30\left(\mathrm{C}_{\mathrm{q}}\right), 123.33$ and $123.25(\mathrm{CHAr}), 121.6$ and 121.3 (CHAr), 118.3 and $118.2(\mathrm{CH}=\mathrm{CHCO}), 111.3$ and $111.1\left(\mathrm{CH}_{\mathrm{Ar}}\right), 74.8$ $\left(C_{1}\right), 68.9\left(C_{3}\right.$ or $\left.C_{5}\right), 66.8\left(C_{5}\right.$ or $\left.C_{3}\right), 56.0(2 \times O M e), 53.1$ (COOMe), 39.8 and $36.8\left(\mathrm{C}_{6}\right.$ and $\left.\mathrm{C}_{2}\right), 35.1\left(\mathrm{C}_{4}\right), 20.7\left(2 \mathrm{xOCOCH}_{3}\right) ; \mathrm{HRMS}-\mathrm{ESI} \mathrm{m} / \mathrm{z}$ $[\mathrm{M}+\mathrm{Na}]^{+}$calcd for $\mathrm{C}_{32} \mathrm{H}_{34} \mathrm{NaO}_{13} 649$ 649.1892; found 649.1865 .

Methyl (3S,5S) 1-hydroxy-3,5 bis[[(2E)-3-(4-acetoxyphenyl)-1-oxo2-propen-1-yl]oxy]cyclohexanecarboxylate 9d. Synthesized according to the general procedure $\mathbf{A}$ using acetylated paracoumaroyl chloride ${ }^{63}$ and methyl 4-deoxy quinate 6 (58mg, 0.305 $\mathrm{mmol}$ ). 9d was obtained as a white solid (142 $\mathrm{mg}$, yield: $82 \%$ ) after purification by flash chromatography on silica using $\mathrm{DCM} / \mathrm{Et}_{2} \mathrm{O} 10 / 1$. $[\alpha]_{D}^{25}=-79.4$ (c $0.83, \mathrm{CH}_{2} \mathrm{Cl}_{2}$ ); m.p. $86-88^{\circ} \mathrm{C} ; \mathrm{IR}(\mathrm{ATR}) 1765,1736$, $1709,1638,1601,1506,1418,1369,1312,1265,1202,1163,1140$, $1086,1063,1011 \mathrm{~cm}^{-1} ;{ }^{1} \mathrm{H}$ NMR $\left(\mathrm{CDCl}_{3}, 295 \mathrm{~K}, 400 \mathrm{MHz}\right) \delta(\mathrm{ppm})=$ 7.67 and $7.62\left(2 \mathrm{~d}, 2 \mathrm{H},{ }^{3} \mathrm{~J} 16.0 \mathrm{~Hz}, \mathrm{CH}=\mathrm{CHCO}\right), 7.52$ and $7.50(2 \mathrm{~d}, 4 \mathrm{H}$, $\left.J_{\text {ortho }} 8.5 \mathrm{~Hz}, \mathrm{H}_{2^{\prime}}\right), 7.10\left(2 \mathrm{~d}, 4 \mathrm{H}, J_{\text {ortho }} 8.5 \mathrm{~Hz}, \mathrm{H}_{3^{\prime}}\right), 6.41$ and $6.33(2 \mathrm{~d}$, $\left.2 \mathrm{H},{ }^{3} \mathrm{~J} 16.0 \mathrm{~Hz}, \mathrm{CH}=\mathrm{CHCO}\right), 5.53$ and $5.48\left(2 \mathrm{~m}, 2 \mathrm{H},{ }^{3} \mathrm{~J} 4.0 \mathrm{~Hz}, \mathrm{H}_{3}\right.$ and $\left.\mathrm{H}_{5}\right), 3.74(\mathrm{~s}, 3 \mathrm{H}, \mathrm{COOMe}), 2.27(\mathrm{~s}, 6 \mathrm{H}, \mathrm{OAc}), 2.24\left(\mathrm{~m}, 1 \mathrm{H}, \mathrm{H}_{4}\right), 2.23-$ $2.19\left(\mathrm{~m}, 2 \mathrm{H}, \mathrm{H}_{2}\right.$ and $\left.\mathrm{H}_{6}\right), 2.11-2.06\left(\mathrm{~m}, 2 \mathrm{H}, \mathrm{H}_{2}\right.$ and $\left.\mathrm{H}_{6}\right), 1.88$ (ddd, $1 \mathrm{H}$, 2) $13.0 \mathrm{~Hz},{ }^{3}$ J $10.2 \mathrm{~Hz},{ }^{3}$ J $\left.3.5 \mathrm{~Hz}, \mathrm{H}_{4}\right) ;{ }^{13} \mathrm{C} \mathrm{NMR}\left(\mathrm{CDCl}_{3}, 295 \mathrm{~K}, 100\right.$ $\mathrm{MHz}) \delta(\mathrm{ppm})=174.9$ (COOMe), $169.2\left(2 \times \mathrm{xOCCH}_{3}\right), 166.1$ and $165.8(\mathrm{COO}), 152.2$ and $152.1\left(\mathrm{C}_{\mathrm{q}}\right), 144.1$ and $144.0(\mathrm{CH}=\mathrm{CHCO})$, 132.1 and $132.0\left(2 \times \mathrm{CH}_{\mathrm{Ar}}\right), 129.4$ and $129.3\left(2 \times \mathrm{CH}_{\mathrm{Ar}}\right), 122.2$ and 122.1 $\left(\mathrm{C}_{\mathrm{q}}\right), 118.3$ and $118.1(\mathrm{CH}=\underline{\mathrm{CHCO}}), 74.7\left(\mathrm{C}_{1}\right), 68.8$ and $66.8\left(\mathrm{C}_{3}\right.$ and $\left.\mathrm{C}_{5}\right), 53.0$ (OMe), 39.7 and $36.8\left(\mathrm{C}_{2}\right.$ and $\left.\mathrm{C}_{6}\right) 35.0\left(\mathrm{C}_{4}\right) 21.1\left(2 \mathrm{xCH}_{3}\right)$; HRMS-ESI m/z [M+Na] ${ }^{+}$calcd for $\mathrm{C}_{30} \mathrm{H}_{30} \mathrm{NaO}_{11}$ 589.1680; found 589.1655.

Methyl (3S,5S) 1-hydroxy-3,5 bis[[(2E)-3-(2-acetoxyphenyl)-1-oxo2-propen-1-yl]oxy]cyclohexanecarboxylate 9e. Synthesized according to the general procedure $\mathbf{A}$ using acetylated orthocoumaroyl chloride and methyl 4-deoxy quinate $6(82 \mathrm{mg}, 0.432$ $\mathrm{mmol})$. Purification by flash chromatography (DCM/Et $\left.{ }_{2} \mathrm{O} 10 / 1\right)$ afforded $9 e$ as a white solid (152 mg, yield: $62 \%) .[\alpha]_{D}^{25}=-50.0(C$ 1.12, $\mathrm{CH}_{2} \mathrm{Cl}_{2}$ ); m.p. $87-90^{\circ} \mathrm{C}$; IR(ATR) 1763, 1711, 1638, 1483, 1454, $1437,1371,1315,1265,1198,1179,1140,1094,1063,1009 \mathrm{~cm}^{-1}$; ${ }^{1} \mathrm{H} \mathrm{NMR}\left(\mathrm{CDCl}_{3}, 296 \mathrm{~K}, 300 \mathrm{MHz}\right) \delta(\mathrm{ppm})=7.81$ and $7.73(2 \mathrm{~d}, 2 \mathrm{H}, J$ $16.1 \mathrm{~Hz}, \mathrm{CH}=\mathrm{CHCO}$ ), 7.63 (dd, $2 \mathrm{H}, J 7.9 \mathrm{~Hz}, \mathrm{H}$ ortho), 7.40 (dd, $2 \mathrm{H}, J$ $7.40 \mathrm{~Hz}, \mathrm{H}$ meta OAc), 7.25 (dd, $2 \mathrm{H}, J 7.6 \mathrm{~Hz}, \mathrm{H}$ para OAc ), 7.11 (d, $3 \mathrm{H}, J 8.1 \mathrm{~Hz}, \mathrm{H}$ orthoOAc), 6.46 and $6.40(2 \mathrm{~d}, 2 \mathrm{H}, J 16.1 \mathrm{~Hz}$, $\mathrm{CH}=\mathrm{CHCO}), 5.53\left(\mathrm{~m}, 2 \mathrm{H}, \mathrm{H}_{3}\right.$ and $\left.\mathrm{H}_{5}\right), 3.75(\mathrm{~s}, 3 \mathrm{H}, \mathrm{COOMe}), 2.40$ and $2.38(2 \mathrm{~s}, 2 \times 3 \mathrm{H}, 2 \times \mathrm{OAc}), 2.29-2.19(\mathrm{~m}, 3 \mathrm{H}, \mathrm{H}-2, \mathrm{H}-4, \mathrm{H}-6), 2.13-2.02$ (m, 2H, H-2, H-6), 1.90 (ddd, 1H, ${ }^{2} \mathrm{~J} 13.5 \mathrm{~Hz},{ }^{3} \mathrm{~J} 10.0 \mathrm{~Hz},{ }^{3} \mathrm{~J} 3.4 \mathrm{~Hz}, \mathrm{H}-$ 4); ${ }^{13} \mathrm{C} \mathrm{NMR}\left(\mathrm{CDCl}_{3}, 295 \mathrm{~K}, 100 \mathrm{MHz}\right) \delta(\mathrm{ppm})=175.0$ (COOMe), 169.45 and $169.40\left(\mathrm{OCOCH}_{3}\right), 165.8$ and $165.6(\mathrm{COO}), 149.4\left(2 \times \mathrm{C}_{\mathrm{q}}\right)$, 138.56 and $138.50(\underline{\mathrm{CH}}=\mathrm{CHCO}), 131.34$ and $131.30\left(\mathrm{C}_{4^{\prime}}\right), 127.6$ and $127.5\left(C_{6^{\prime}}\right), 127.1$ and $127.0\left(C_{q}\right), 126.44$ and $126.41\left(C_{5^{\prime}}\right), 123.26$ and $123.20\left(\mathrm{C}_{3^{\prime}}\right), 120.2$ and $120.1(\mathrm{CH}=\mathrm{CHCO}), 74.6\left(\mathrm{C}_{1}\right), 68.8\left(\mathrm{C}_{3}\right.$ or $\left.\mathrm{C}_{5}\right)$, $67.0\left(C_{5}\right.$ or $\left.C_{3}\right), 53.2$ (COOMe), 39.7 and $36.9\left(C_{6}\right.$ and $\left.C_{2}\right), 35.1\left(C_{4}\right)$, $21.0\left(2 \mathrm{x} \quad \mathrm{OCOCH}_{3}\right) ; \quad \mathrm{HRMS}-\mathrm{ESI} \quad \mathrm{m} / \mathrm{z} \quad[\mathrm{M}+\mathrm{Na}]^{+}$calcd for $\mathrm{C}_{30} \mathrm{H}_{30} \mathrm{NaO}_{11}$ 589.1680; found 589.1669.

General procedure for the deprotection of aromatic acetates Procedure $D^{55}$ : To a solution of compound 9 in ethanol/water (2/1, 
$0.12 \mathrm{M}$ ) was added hydrazine acetate ( $\mathrm{NaOAc}, 20 \mathrm{eq})$. After stirring at reflux for $1 \mathrm{~h}$, the mixture was diluted with EtOAc, washed with brine then the organic layer was dried over $\mathrm{MgSO}_{4}$, concentrated and purified by flash chromatography.

Procedure $\mathrm{E}^{56}$ : To a solution of compound $\mathbf{9}$ (a, c, d or e) in DMF $(0.15 \mathrm{M})$ was added hydrazine acetate (75 equiv.). After stirring at room temperature for $1 \mathrm{~h}$, the mixture was diluted with EtOAc, washed with brine then the organic layer was dried over $\mathrm{MgSO}_{4}$, concentrated and purified by flash chromatography.

Methyl (3S,5S) 1-hydroxy-3,5 bis[[(2E)-3-(3,4-dihydroxyphenyl)-1oxo-2-propen-1-yl]oxy]cyclohexanecarboxylate 10a. The general procedure $\mathbf{E}$ was performed with $9 \mathrm{a}(116 \mathrm{mg}, 0.170 \mathrm{mmol})$ in DMF $(0.9 \mathrm{~mL})$ and hydrazine acetate $(71 \mathrm{mg}, 0.64 \mathrm{mmol})$ providing after purification 10a as a colorless oil $(78 \mathrm{mg}, 89 \%)$. $[\alpha]_{D}^{25}=-167.0$ (c $0.64, \mathrm{CH}_{3} \mathrm{OH}$ ); IR(ATR) 3323, 3304, 3285, 3275, 2949, 2835, 1680, 1630, 1599, 1516, 1443, 1373, 1256, 1159, 1140, 1119, 1067, 1016 $\mathrm{cm}^{-1} ;{ }^{1} \mathrm{H}$ NMR $\left(\mathrm{CD}_{3} \mathrm{OD}, 298 \mathrm{~K}, 500 \mathrm{MHz}\right): \delta(\mathrm{ppm})=7.58$ and $7.52(\mathrm{~d}$, $2 \mathrm{H},{ }^{3} \mathrm{~J} 15.8 \mathrm{~Hz}, \mathrm{CH}=\mathrm{CHCO}$ ), 7.06 (bs, $\left.2 \mathrm{H}, \mathrm{Ar}-\mathrm{H}_{2^{\prime}}\right), 6.95$ (dt, $2 \mathrm{H}, J_{\text {ortho }}=$ $\left.8.1, J_{\text {meta }} 2.3 \mathrm{~Hz}, \mathrm{Ar}-\mathrm{H}_{6^{\prime}}\right), 6.80\left(\mathrm{dd}, 2 \mathrm{H}, J_{\text {ortho }} 8,2 \mathrm{~Hz}, J_{\text {meta }} 2,7 \mathrm{~Hz}, \mathrm{Ar}-\right.$ $\left.\mathrm{H}_{5^{\prime}}\right), 6.28$ and $6.19\left(\mathrm{~d}, 2 \mathrm{H},{ }^{3} \mathrm{~J} 15.9 \mathrm{~Hz}, \mathrm{CH}=\mathrm{CHCO}\right), 5.42\left(\mathrm{~m}, 2 \mathrm{H}, \mathrm{H}_{3}\right.$ and $\mathrm{H}_{5}$ ), 3.70 (s, 3H, OMe), 2.46 (dd, $1 \mathrm{H},{ }^{2}$ J $13.4 \mathrm{~Hz},{ }^{3}$ J $3.5 \mathrm{~Hz}, \mathrm{H}_{2}$ or $\mathrm{H}_{6}$ ), $2.39\left(\mathrm{dd}, 1 \mathrm{H},{ }^{2} \mathrm{~J} 13.6 \mathrm{~Hz},{ }^{3}\right.$ J $6.9 \mathrm{~Hz}, \mathrm{H}_{2}$ or $\left.\mathrm{H}_{6}\right), 2.06\left(\mathrm{~m}, 1 \mathrm{H}, \mathrm{H}_{4}\right), 1.97$ (m, $2 \mathrm{H}, \mathrm{H}_{4}$ and $\mathrm{H}_{2}$ or $\mathrm{H}_{6}$ ), 1.86 (dd, $1 \mathrm{H},{ }^{2}$ J $13.4 \mathrm{~Hz},{ }^{3}$ J 7.7 Hz, $\mathrm{H}_{2}$ or $\mathrm{H}_{6}$ ); ${ }^{13} \mathrm{C} \mathrm{NMR}\left(\mathrm{CD}_{3} \mathrm{OD}, 298 \mathrm{~K}, 125 \mathrm{MHz}\right) \delta(\mathrm{ppm})=175.7$ (COOMe), 168.5 (OCO), 168.0 (OCO), $149.6\left(C_{q}\right), 149.5\left(C_{q}\right), 147.2$ and 147.0 $(\underline{\mathrm{CH}}=\mathrm{CHCO}), 146.8\left(\mathrm{C}_{\mathrm{q}}\right), 146.7\left(\mathrm{C}_{\mathrm{q}}\right), 127.7\left(\mathrm{C}_{\mathrm{q}}\right), 127.6\left(\mathrm{C}_{\mathrm{q}}\right), 123.04$ and $123.00\left(\mathrm{C}_{6^{\prime}}\right), 116.53$ and $116.50\left(\mathrm{C}_{5^{\prime}}\right), 115.4$ and $115.1(\mathrm{OCOCH}=\underline{\mathrm{CH}})$, 115.09 and $115.00\left(C_{2^{\prime}}\right), 75.0\left(C_{1}\right), 69.5$ and $69.0\left(C_{3}\right.$ and $\left.C_{5}\right)$, 53.0(OMe), 39.7, 39.3 and $35.9\left(3 \mathrm{xCH}_{2}, \mathrm{C}_{2}, \mathrm{C}_{6}\right.$ and $\left.\mathrm{C}_{4}\right)$; HRMS-ESI m/z $[\mathrm{M}+\mathrm{Na}]^{+}$calcd for $\mathrm{C}_{26} \mathrm{H}_{26} \mathrm{NaO}_{11}$ 537.1367; found 537.1351.

Methyl (3S,5S) 1-hydroxy-3,5 bis[[(2E)- 3-(4-hydroxy-3methoxyphenyl)-1-oxo-2-propen-1-yl]oxy]cyclohexanecarboxylate 10c. The general procedure $\mathbf{E}$ was performed with $9 \mathrm{c}(65 \mathrm{mg}, 0.104$ $\mathrm{mmol})$ in DMF $(0.45 \mathrm{~mL}$ ) and hydrazine acetate $(43 \mathrm{mg}, 0.47 \mathrm{mmol})$ providing after purification $10 \mathrm{c}$ as a colorless oil (50 $\mathrm{mg}, 89 \%$ ). $[\alpha]_{D}^{25}=-170.0$ (c 0.93, $\mathrm{CH}_{3} \mathrm{OH}$ ); IR(ATR) 3516, 3057, 2957, 2941, $1701,1631,1593,1512,1462,1454,1429,1377,1263,1207,1157$, 1138, 1032, $1009 \mathrm{~cm}^{-1} ;{ }^{1} \mathrm{H}$ NMR $\left(\mathrm{CD}_{3} \mathrm{OD}, 400 \mathrm{MHz}\right): \delta \mathrm{ppm}=7.63$ and 7.57 (d, 2H, ${ }^{3} \mathrm{~J} 15.9 \mathrm{~Hz}, \mathrm{CH}=\mathrm{CHCO}$ ), 7.12 (bs, 2H, Ar-H), 7.07 (dd, $\left.2 \mathrm{H}, J_{\text {ortho }} 8,2 \mathrm{~Hz}, J_{\text {meta }} 2,7 \mathrm{~Hz}, \mathrm{Ar}-\mathrm{H}\right), 6.81$ (dd, $2 \mathrm{H}, J_{\text {ortho }} 8,2 \mathrm{~Hz}, J_{\text {meta }}$ 2,2 Hz, Ar-H), 6.38 and 6.29 (d, $\left.2 \mathrm{H},{ }^{3} \mathrm{~J} 15.9 \mathrm{~Hz}, \mathrm{CH}=\mathrm{CHCO}\right), 5.432(\mathrm{~m}$, $2 \mathrm{H}, \mathrm{H}_{3}$ and $\mathrm{H}_{5}$ ), 3.88 (s, 6H, 2x OMe), 3.70 (s, 3H, COOMe), 2.45 (dd, $1 \mathrm{H},{ }^{2} \mathrm{~J} 13.6 \mathrm{~Hz},{ }^{3}$ J $4.1 \mathrm{~Hz}, \mathrm{H}_{2}$ or $\left.\mathrm{H}_{6}\right), 2.38\left(\mathrm{dd}, 1 \mathrm{H},{ }^{2} \mathrm{~J} 13.6 \mathrm{~Hz},{ }^{3} \mathrm{~J} 7.2 \mathrm{~Hz}\right.$, $\mathrm{H}_{2}$ or $\left.\mathrm{H}_{6}\right), 2.14-1.97\left(\mathrm{~m}, 3 \mathrm{H}, 2 \mathrm{x} \mathrm{H}_{4}\right.$ and $\mathrm{H}_{2}$ or $\left.\mathrm{H}_{6}\right), 1.88\left(\mathrm{dd}, 1 \mathrm{H},{ }^{2} \mathrm{~J} 13.6\right.$ $\mathrm{Hz},{ }^{3} \mathrm{~J} 7.5 \mathrm{~Hz}, \mathrm{H}_{2}$ or $\left.\mathrm{H}_{6}\right) ;{ }^{13} \mathrm{C} \mathrm{NMR}\left(\mathrm{CD}_{3} \mathrm{OD}, 100 \mathrm{MHz}\right) \delta(\mathrm{ppm})=175.7$ (COOMe), 168.4 and 168.0 (OCO), 150.7, 150.6, 149.4, 149.3 ( $\mathrm{C}_{\mathrm{q} \mathrm{Ar}}$ ), 147.1 and $147.0(\mathrm{CH}=\mathrm{CHCO}), 127.7$ and $127.5\left(\mathrm{C}_{\mathrm{q}} \mathrm{Ar}\right), 124.2$ and $124.1\left(\mathrm{CH}_{\mathrm{Ar}}\right), 116.5$ and $116.4\left(\mathrm{CH}_{\mathrm{Ar}}\right), 115.7$ and $115.4(\mathrm{OCOCH}=\mathrm{CH})$, $111.6\left(\mathrm{CH}_{\mathrm{Ar}}\right), 75.0\left(\mathrm{C}_{1}\right), 69.5$ and $69.0\left(\mathrm{C}_{5}\right.$ or $\left.\mathrm{C}_{3}\right), 56.4(2)\left(2 \mathrm{xOCH}_{3}\right)$, 53.0 (COOMe), 39.8 and $39.2\left(C_{2}\right.$ or $\left.C_{6}\right), 35.9\left(C_{4}\right)$; HRMS-ESI m/z $[\mathrm{M}+\mathrm{Na}]^{+}$calcd for $\mathrm{C}_{28} \mathrm{H}_{30} \mathrm{NaO}_{11} 65.1680$; found 565.1662.

Methyl (3S,5S) 1-hydroxy-3,5 bis[[(2E)-3-(4-hydroxyphenyl)-1-oxo2-propen-1-yl]oxy]cyclohexanecarboxylate 10d. The general procedure $\mathbf{E}$ was performed with $9 \mathbf{d}(80 \mathrm{mg}, 0.14 \mathrm{mmol})$ in DMF $(0.6 \mathrm{~mL})$ and hydrazine acetate $(59 \mathrm{mg}, 0.64 \mathrm{mmol})$ providing after purification $\left(\mathrm{CH}_{2} \mathrm{Cl}_{2} / \mathrm{CH}_{3} \mathrm{OH} 40 / 1\right) 10 \mathrm{~d}$ as a colorless oil $(62 \mathrm{mg}$, 91\%). $[\alpha]_{D}^{25}=-174.7$ (c $0.95, \mathrm{CH}_{3} \mathrm{OH}$ ); IR(ATR) 3325, 3308, 3279, $3267,2947,2832,1688,1632,1603,1587,1514,1441,1371,1325$, $1307,1254,1202,1165,1144,1103,1065,1018 \mathrm{~cm}^{-1} ;{ }^{1} \mathrm{H}$ NMR
$\left(\mathrm{CD}_{3} \mathrm{OD}, 400 \mathrm{MHz}\right): \delta(\mathrm{ppm})=7.65$ and $7.59\left(2 \mathrm{~d}, 2 \mathrm{H},{ }^{3} \mathrm{~J} 15.9 \mathrm{~Hz}, 2 \mathrm{x}\right.$ $\mathrm{CH}=\mathrm{CHCO}), 7.47\left(\mathrm{~d}, 4 \mathrm{H}, J_{\text {ortho }} 8.6 \mathrm{~Hz}, \mathrm{Ar}-\mathrm{H}\right), 6.81\left(\mathrm{dd}, 4 \mathrm{H}, J_{\text {ortho }} 8.6 \mathrm{~Hz}\right.$, $\left.J_{\text {meta }} 1.8 \mathrm{~Hz} \mathrm{Ar}-\mathrm{H}\right), 6.34$ and $6.26\left(\mathrm{~d}, 2 \mathrm{H},{ }^{3} \mathrm{~J} 15.9 \mathrm{~Hz}, \mathrm{CH}=\mathrm{CHCO}\right), 5.43$ $\left(\mathrm{m}, 2 \mathrm{H}, \mathrm{H}_{3}\right.$ and $\left.\mathrm{H}_{5}\right), 3.69(\mathrm{~s}, 3 \mathrm{H}, \mathrm{OMe}), 2.46\left(\mathrm{dd}, 1 \mathrm{H},{ }^{2} \mathrm{~J} 13.4 \mathrm{~Hz},{ }^{3} \mathrm{~J} 4.1\right.$ $\mathrm{Hz}, \mathrm{H}_{2}, \mathrm{H}_{4}$ or $\left.\mathrm{H}_{6}\right), 2.40$ (dd, $1 \mathrm{H},{ }^{2} J 13.7 \mathrm{~Hz},{ }^{3} \mathrm{~J} 7.0 \mathrm{~Hz}, \mathrm{H}_{2}, \mathrm{H}_{4}$ or $\mathrm{H}_{6}$ ), 2.10-1.95 (m, $3 \mathrm{H}, \mathrm{H}_{2}, \mathrm{H}_{4}$ or $\left.\mathrm{H}_{6}\right), 1.87$ (dd, $1 \mathrm{H},{ }^{2}$ J $13.4 \mathrm{~Hz},{ }^{3} \mathrm{~J} 7.6 \mathrm{~Hz} \mathrm{H}_{2}$, $\mathrm{H}_{4}$ or $\left.\mathrm{H}_{6}\right) .{ }^{13} \mathrm{C}$ NMR $\left(\mathrm{CD}_{3} \mathrm{OD}, 100 \mathrm{MHz}\right) \delta(\mathrm{ppm})=175.7$ (COOMe), 168.5(OCO), 168.0(OCO), $161.4\left(\mathrm{C}_{\mathrm{q}}\right), 161.3\left(\mathrm{C}_{\mathrm{q}}\right), 146.8$ and 146.7 $(2 \times \underline{C H}=\mathrm{CHCO}), 131.23$ and $131.20(2 \times 2 \times C H), 127.2$ and $127.0\left(2 \times \mathrm{C}_{\mathrm{q}}\right)$, 116.9, $116.8(2 \times 2 \mathrm{CH}), 115.5$ and $115.1(2 \times \mathrm{OCOCH}=\mathrm{CH}), 75.0\left(\mathrm{C}_{1}\right)$, 69.5, $69.0\left(\mathrm{C}_{3}\right.$ and $\left.\mathrm{C}_{5}\right), 53.0$ (OMe), 39.8, 39.3 and $35.9\left(3 \times \mathrm{CH}_{2}, \mathrm{C}_{2}, \mathrm{C}_{6}\right.$ and $\mathrm{C}_{4}$ ). HRMS-ESI m/z $[\mathrm{M}+\mathrm{Na}]^{+}$calcd for $\mathrm{C}_{26} \mathrm{H}_{26} \mathrm{NaO}_{9} 505.1469$; found 505.1452 .

Methyl (3S,5S) 1-hydroxy-3,5 bis[[(2E)-3-(2-hydroxyphenyl)-1-oxo2-propen-1-yl]oxy]cyclohexanecarboxylate 10e. The general procedure $\mathbf{E}$ was performed with $9 \mathrm{e}(118 \mathrm{mg}, 0.21 \mathrm{mmol})$ in DMF $(0.9 \mathrm{~mL})$ and hydrazine acetate $(88 \mathrm{mg}, 0.96 \mathrm{mmol})$ providing after purification $\left(\mathrm{CH}_{2} \mathrm{Cl}_{2} / \mathrm{CH}_{3} \mathrm{OH} 40 / 1\right) \mathbf{1 0 e}$ as a colorless oil $(89 \mathrm{mg}$, 89\%). $[\alpha]_{D}^{25}=-104.3$ (c $1.00, \mathrm{CH}_{3} \mathrm{OH}$ ); IR(ATR) 3337, 3298, 3283, $3256,2945,2835,1688,1626,1603,1499,1458,1369,1317,1294$, 1254, 1169, 1146, 1113, 1067, $1018 \mathrm{~cm}^{-1} ;{ }^{1} \mathrm{H}$ NMR ( $\mathrm{CD}_{3} \mathrm{OD}, 400$ $\mathrm{MHz}): \delta(\mathrm{ppm})=$ contaminated with chloroform at $7.90 \mathrm{ppm}, 8.00$ and $7.91\left(2 \mathrm{~d}, 2 \mathrm{H},{ }^{3} J 16.1 \mathrm{~Hz}, \mathrm{CH}=\mathrm{CHCO}\right.$ ), 7.48 (pseudo $\mathrm{t}, 2 \mathrm{H}, J_{\text {ortho }} 8,2$ $\mathrm{Hz}$ Ar-H), 7.21 (pseudot, $\left.2 \mathrm{H}, J_{\text {ortho }} 7.4 \mathrm{~Hz} \mathrm{Ar}-\mathrm{H}\right), 6.84(\mathrm{~m}, 4 \mathrm{H}, \mathrm{Ar}-\mathrm{H})$, 6.63 and $6.58\left(\mathrm{~d}, 2 \mathrm{H},{ }^{3} \mathrm{~J} 16.1 \mathrm{~Hz}, \mathrm{CH}=\mathrm{CHCO}\right), 5.45\left(\mathrm{~m}, 2 \mathrm{H}, \mathrm{H}_{3}\right.$ and $\left.\mathrm{H}_{5}\right)$, $3.72(\mathrm{~s}, 3 \mathrm{H}, \mathrm{OMe}), 2.49$ (dd, $1 \mathrm{H},{ }^{2} \mathrm{~J} 13.4 \mathrm{~Hz},{ }^{3}$ J $3.6 \mathrm{~Hz}, \mathrm{H}_{2}, \mathrm{H}_{4}$ or $\mathrm{H}_{6}$ ), 2.43 (dd, $1 \mathrm{H}^{2}{ }^{2} \mathrm{~J} 13.8 \mathrm{~Hz},{ }^{3} \mathrm{~J} 6.8 \mathrm{~Hz}, \mathrm{H}_{2}, \mathrm{H}_{4}$ or $\left.\mathrm{H}_{6}\right), 2.15-2.09\left(\mathrm{~m}, 1 \mathrm{H}, \mathrm{H}_{2}\right.$, $\mathrm{H}_{4}$ or $\left.\mathrm{H}_{6}\right), 2.00-1.96\left(\mathrm{~m}, 2 \mathrm{H}, \mathrm{H}_{2}, \mathrm{H}_{4}\right.$ or $\left.\mathrm{H}_{6}\right), 1.86\left(\mathrm{dd}, 1 \mathrm{H},{ }^{2} \mathrm{~J} 13.3 \mathrm{~Hz},{ }^{3} \mathrm{~J}\right.$ $7.9 \mathrm{~Hz}, \mathrm{H}_{2}, \mathrm{H}_{4}$ or $\left.\mathrm{H}_{6}\right) .{ }^{13} \mathrm{C} \operatorname{NMR}\left(\mathrm{CD}_{3} \mathrm{OD}, 100 \mathrm{MHz}\right) \delta(\mathrm{ppm})=$ contaminated with chloroform at $79.4 \mathrm{ppm} ; 175.7$ (COOMe), 168.8(OCO), 168.4(OCO), 158.5 and $158.3\left(2 \mathrm{xC}_{\mathrm{q}}\right), 142.9$ and 142.5 $(2 \times \underline{C H}=\mathrm{CHCO}), 132.7,132.6,130.6,130.2\left(4 \mathrm{xCH}_{\mathrm{Ar}}\right), 122.6,122.5$ $\left(2 \times \mathrm{C}_{\mathrm{q}}\right), 120.8\left(2 \times \mathrm{CH}_{\mathrm{Ar}}\right), 118.6,118.5(2 \times \mathrm{OCOCH}=\mathrm{CH}), 117.0\left(2 \times \mathrm{CH}_{\mathrm{Ar}}\right)$, 79.4, $75.0\left(C_{1}\right), 69.5,69.1\left(C_{3}\right.$ and $\left.C_{5}\right), 53.0$ (OMe), 39.8, 39.4 and $35.9\left(3 \mathrm{xCH}_{2}, \mathrm{C}_{2}, \mathrm{C}_{6}\right.$ and $\left.\mathrm{C}_{4}\right)$. HRMS-ESI $\mathrm{m} / \mathrm{z}[\mathrm{M}+\mathrm{Na}]^{+}$calcd for $\mathrm{C}_{26} \mathrm{H}_{26} \mathrm{NaO}_{9}$ 505.1469; found 505.1448.

$(3 S, 5 S)$ 1-hydroxy-3,5 bis[[(2E)-3-(3,4-dihydroxyphenyl)-1-oxo-2propen-1-yl]oxy]cyclohexanecarboxylic acid 4a. Lil (143 mg, 1.07 $\mathrm{mmol}$ ) was added to a solution of 9 a $(146 \mathrm{mg}, 0.214 \mathrm{mmol})$ in EtOAc $(1.1 \mathrm{~mL})$ then the mixture was heated under reflux overnight. After cooling down to room temperature, the mixture was diluted with EtOAc $(30 \mathrm{~mL})$, washed with $1 \mathrm{M} \mathrm{HCl}(2 \times 10 \mathrm{~mL})$ and extracted with EtOAc $(3 \times 30 \mathrm{~mL})$. The organic layer was dried over $\mathrm{MgSO}_{4}$, concentrated to give the crude $(3 \mathrm{~S}, 5 \mathrm{~S})$ 1-hydroxy-3,5 bis[[(2E)-3(3,4-diacetoxyphenyl)-1-oxo-2-propen-1-

yl]oxy]cyclohexanecarboxylic acid 11a (128 mg, crude yield: 90\%), pure enough for characterization and used directly in the following step. IR(ATR) 3370, 2941, 2833, 1769, 1707, 1638, 1504, 1369, 1240, 1201, 1174, 1143, 1109, $1012 \mathrm{~cm}^{-1} ;{ }^{1} \mathrm{H}$ NMR (CD 3 OD, 298K, $400 \mathrm{MHz}): \delta(\mathrm{ppm})=7.67$ and $7.62(2 \mathrm{~d}, 2 \mathrm{H}, J 16.0 \mathrm{~Hz}, \mathrm{C} \underline{\mathrm{H}}=\mathrm{CHCO})$, $7.50\left(\mathrm{~m}, 2 \mathrm{H}, \mathrm{H}_{6^{\prime}}\right), 7.49\left(\mathrm{~m}, 2 \mathrm{H}, \mathrm{H}_{2^{\prime}}\right), 7.24\left(\mathrm{~d}, 2 \mathrm{H}, J_{\text {ortho }} 8.2 \mathrm{~Hz}, \mathrm{H}_{5}{ }^{\prime}\right)$ ), 6.52 and $6.46(2 \mathrm{~d}, 2 \mathrm{H}, J 16.0 \mathrm{~Hz}, \mathrm{CH}=\mathrm{CHCO}), 5.46\left(\mathrm{~m}, 2 \mathrm{H}, \mathrm{H}_{3}\right.$ and $\left.\mathrm{H}_{5}\right)$, 2.44-2.19 $(\mathrm{m}, 14 \mathrm{H})$ and $2.06-1.90\left(\mathrm{~m}, \overline{4 \mathrm{H}}, 3 \mathrm{xCH}_{2}\right.$ and $\left.4 \mathrm{xOCOCH}_{3}\right) ;{ }^{13} \mathrm{C}$ NMR $\left(\mathrm{CD}_{3} \mathrm{OD}, 298 \mathrm{~K}, 100 \mathrm{MHz}\right) \delta(\mathrm{ppm})=177.3(\mathrm{COOH}), 169.8$ and $169.7\left(4 \times \mathrm{OCOCH}_{3}\right), 167.5$ and 167.2 (2xOCO), 145.2 and 145.1 $\left(2 \times C_{q}\right), 144.5$ and $144.4(2 \times C H=C H C O), 144.0\left(2 \times C_{q}\right), 134.60$ and $134.56\left(2 \times \mathrm{C}_{\mathrm{q}}\right), 127.7$ and $127.6\left(2 \times \mathrm{C}_{6^{\prime}}\right), 125.13$ and $125.11\left(2 \times \mathrm{C}_{5^{\prime}}\right)$, 124.14 and $124.09\left(\mathrm{C}_{2^{\prime}}\right), 120.45$ and $120.13(2 \times \mathrm{CH}=\mathrm{CHCO}), 75.0\left(\mathrm{C}_{1}\right)$, 70.2, $69.2\left(\mathrm{C}_{3}\right.$ and $\left.\mathrm{C}_{5}\right), 39.9,38.7,35.9\left(\mathrm{C}_{2}, \mathrm{C}_{4}, \mathrm{C}_{6}\right), 20.5\left(4 \mathrm{XOCOCH}_{3}\right)$. 
HRMS-ESI m/z $[\mathrm{M}+\mathrm{H}]^{+}$calcd for $\mathrm{C}_{33} \mathrm{H}_{33} \mathrm{O}_{15} 669.1814$; found 669.1801.

To a solution of (3S,5S) 1-hydroxy-3,5 bis[[(2E)-3-(3,4diacetoxyphenyl)-1-oxo-2-propen-1-yl]oxy]cyclohexanecarboxylic acid 11a (208 $\mathrm{mg}$ ) in acetone $(4.8 \mathrm{~mL})$ was added $3 \mathrm{M} \mathrm{HCl}$ aqueous solution $(2.0 \mathrm{~mL})$ and the mixture was refluxed for $5 \mathrm{~h}$. After completion, the reaction mixture was cooled down to room temperature, diluted with EtOAc $(30 \mathrm{~mL})$ and washed with brine $(20$ $\mathrm{mL}$ ). The organic layer was concentrated to give the crude quantitatively as a pale solid 4 a $\left(190 \mathrm{mg}\right.$ ). $[\alpha]_{D}^{25}=-42.7$ (c 0.82, $\mathrm{CH}_{3} \mathrm{OH}$ ); IR(ATR) 3345, 3210, 1687, 1603, 1523, 1445, 1362, 1279, $1182,1138,1119 \mathrm{~cm}^{-1} ;{ }^{1 \mathrm{H}} \mathrm{NMR}\left(\mathrm{CD}_{3} \mathrm{OD}, 400 \mathrm{MHz}, 295 \mathrm{~K}\right): \delta(\mathrm{ppm})=$ 7.65 and $7.60(2 \mathrm{~d}, 2 \mathrm{H}, J 16.0 \mathrm{~Hz}, \mathrm{CH}=\mathrm{CHCO}), 7.45(\mathrm{~m}, 4 \mathrm{H}, \mathrm{ArH}), 6.81$ (m, $4 \mathrm{H}, \mathrm{ArH}), 6.34$ and $6.27(2 \mathrm{~d}, 2 \mathrm{H}, J 16.0 \mathrm{~Hz}, \mathrm{CH}=\mathrm{CHCO}), 5.45(\mathrm{~m}$, $2 \mathrm{H}, \mathrm{H}_{3}$ and $\mathrm{H}_{5}$ ), $2.41\left(\mathrm{dd}, 1 \mathrm{H},{ }^{2} \mathrm{~J} 14.0 \mathrm{~Hz}^{3}{ }^{3} 3.8 \mathrm{~Hz}, \mathrm{H}_{2}, \mathrm{H}_{4}\right.$ or $\left.\mathrm{H}_{6}\right), 2.32$ (dd, $1 \mathrm{H},{ }^{2}$ J $13.3 \mathrm{~Hz},{ }^{3}$ J $8.2 \mathrm{~Hz}, \mathrm{H}_{2}, \mathrm{H}_{4}$ or $\left.\mathrm{H}_{6}\right), 2.06\left(\mathrm{~m}, 3 \mathrm{H}, \mathrm{H}_{2}, \mathrm{H}_{4}\right.$ or $\mathrm{H}_{6}$ ), $1.93\left(\mathrm{dd}, 1 \mathrm{H},{ }^{2}\right.$ J $13.8 \mathrm{~Hz},{ }^{3} \mathrm{~J} 6.5 \mathrm{~Hz}, \mathrm{H}_{2}, \mathrm{H}_{4}$ or $\left.\mathrm{H}_{6}\right) ;{ }^{13} \mathrm{C} \mathrm{NMR}\left(\mathrm{CD}_{3} \mathrm{OD}\right.$, $100 \mathrm{MHz}, 297 \mathrm{~K}) \delta(\mathrm{ppm})=177.4(\mathrm{COOH}), 171.0\left(2 \times \mathrm{C}_{\mathrm{q}}\right), 168.5$ and 168.3 (2xOCO), 161.12 and $161.07(2 \times C q), 146.7$ and 146.7 $(2 \times \underline{C H}=\mathrm{CHCO}), 131.2,131.1\left(2 \times \mathrm{CH}_{\mathrm{Ar}}\right), 127.1\left(2 \times \mathrm{C}_{\mathrm{q}}\right), 116.8(4 \times \mathrm{CHAr})$, 115.5, 115.1 (2x CH=CHCO), $75.1\left(C_{1}\right), 69.9,68.7\left(C_{3}\right.$ and $\left.C_{5}\right), 39.9$, 38.6, $35.9\left(\mathrm{C}_{2}, \mathrm{C}_{4}, \mathrm{C}_{6}\right)$.

(3S,5S) 1-hydroxy-3,5 bis[[(2E)-3-(4-hydroxy-3-methoxyphenyl)-1oxo-2-propen-1-yl]oxy]cyclohexanecarboxylic acid 4c. Lil (152 mg, $1.135 \mathrm{mmol})$ was added to a solution of $9 \mathrm{c}(142 \mathrm{mg}, 0.2274 \mathrm{mmol})$ in EtOAc $(5.0 \mathrm{~mL})$ then the mixture was heated under reflux $24 \mathrm{~h}$. After cooling down to room temperature, the mixture was diluted with EtOAc $(25 \mathrm{~mL})$, washed with $3 \mathrm{M} \mathrm{HCl}(5 \mathrm{~mL})$ and extracted with EtOAc $(3 \times 30 \mathrm{~mL})$. The organic layer was dried over $\mathrm{MgSO}_{4}$, concentrated to give the crude $(3 \mathrm{~S}, 5 \mathrm{~S})$ 1-hydroxy-3,5 bis[[(2E)-3-(4acetoxy-3-methoxyphenyl)-1-oxo-2-propen-1-

yl]oxy]cyclohexanecarboxylic acid 11c (quantitative crude yield), pure enough for characterization and used directly in the following step. $[\alpha]_{D}^{25}=-97.5$ (c $0.96, \mathrm{CH}_{3} \mathrm{OH}$ ); IR(ATR) 3466, 2941, 2833, 1763, 1705, 1599, 1508, 1464, 1454, 1418, 1369, 1256, 1215, 1177, 1152, 1063, $1020 \mathrm{~cm}^{-1} ;{ }^{1} \mathrm{H}$ NMR $\left(\mathrm{CDCl}_{3}, 400 \mathrm{MHz}\right): \delta(\mathrm{ppm})=7.66$ and $7.61\left(2 \mathrm{~d}, 2 \mathrm{H},{ }^{3} \mathrm{~J} 15.9 \mathrm{~Hz}, \mathrm{CH}=\mathrm{CHCO}\right), 7.13-7.03(2 \mathrm{~m}, 6 \mathrm{H}, \mathrm{Ar}-\mathrm{H}), 6.41$ and $6.34\left(2 \mathrm{~d}, 2 \mathrm{H},{ }^{3} \mathrm{~J} 15.9 \mathrm{~Hz}, \mathrm{CH}=\mathrm{CHCO}\right), 5.50\left(\mathrm{~m}, 2 \mathrm{H}, \mathrm{H}_{3}\right.$ and $\left.\mathrm{H}_{5}\right)$, 3.87 and $3.85(2 \mathrm{~s}, 2 \times 3 \mathrm{H}, 2 \times \mathrm{OMe}), 2.33-1.83\left(\mathrm{~m}, 12 \mathrm{H}, \mathrm{H}_{2}, \mathrm{H}_{4}, \mathrm{H}_{6}\right.$ and $\left.2 \times \mathrm{OCOCH}_{3}\right) ;{ }^{13} \mathrm{C} \mathrm{NMR}\left(\mathrm{CDCl}_{3}, 100 \mathrm{MHz}\right) \delta(\mathrm{ppm})=176.7(\mathrm{COOH})$, 169.04 and $169.02\left(2 \times \mathrm{OCOCH}_{3}\right), 166.1$ and 166.0 (2xCOO), 151.53 and $151.50\left(2 \times C_{q}\right), 145.3,144.8(2 \times \underline{C H}=\mathrm{CHCO}), 141.7,141.6\left(2 \times \mathrm{C}_{\mathrm{q}}\right)$, 133.3, $133.2\left(2 \times \mathrm{C}_{\mathrm{q}}\right), 123.4\left(2 \times \mathrm{CH}_{\mathrm{Ar}}\right), 121.8,121.4(2 \times \mathrm{CH}=\underline{\mathrm{CHCO}})$, $118.1,117.9\left(2 \times \mathrm{CH}_{\mathrm{Ar}}\right), 111.4,111.3\left(2 \times \mathrm{CH}_{\mathrm{Ar}}\right), 75.2\left(\mathrm{C}_{1}\right), 69.4,66.5\left(\mathrm{C}_{3}\right.$ and $\left.C_{5}\right), 56.1,56.0(2 \times O M e), 39.3,36.5,34.9\left(C_{2}, C_{4}, C_{6}\right), 20.8$ $\left(2 \times \mathrm{OCOCH}_{3}\right) ; \quad \mathrm{HRMS}-\mathrm{ESI} \mathrm{m} / \mathrm{z} \quad[\mathrm{M}+\mathrm{Na}]^{+}$calcd for $\mathrm{C}_{31} \mathrm{H}_{32} \mathrm{NaO}_{13}$ 635.1735; found 635.1706.

To a solution of $(3 S, 5 S)$ 1-hydroxy-3,5 bis[[(2E)-3-(4-acetoxy-3methoxyphenyl)-1-oxo-2-propen-1-yl]oxy]cyclohexanecarboxylic acid $11 \mathrm{c}(176 \mathrm{mg})$ in acetone $(3.0 \mathrm{~mL})$ was added $3 \mathrm{M} \mathrm{HCl}$ aqueous solution $(1.5 \mathrm{~mL})$ and the mixture was refluxed for $4 \mathrm{~h}$. After completion, the reaction mixture was cooled down to room temperature, diluted with EtOAc $(30 \mathrm{~mL})$ and washed with brine $(20$ $\mathrm{mL}$ ). The organic layer was concentrated then dissolved in $\mathrm{MeOH} / \mathrm{H}_{2} \mathrm{O}(30 / 40 \mathrm{~mL})$ and washed with pentane $(2 \times 15 \mathrm{~mL})$. After concentration, the crude was obtained as a pale solid $4 \mathrm{c}(150 \mathrm{mg})$. $[\alpha]_{D}^{25}=-94.4$ (c $0.67, \mathrm{CH}_{3} \mathrm{OH}$ ); IR(ATR) 3356, 3277, 1684, 1632, $1605,1514,1443,1327,1261,1204,1171,1138,1007 \mathrm{~cm}^{-1} ;{ }^{1} \mathrm{H}$ NMR ( $\left.\mathrm{CD}_{3} \mathrm{OD}, 300 \mathrm{MHz}, 297 \mathrm{~K}\right): \delta(\mathrm{ppm}) 7.65$ and $7.60(2 \mathrm{~d}, 2 \mathrm{H}, J$
$15.9 \mathrm{~Hz}, \mathrm{CH}=\mathrm{CHCO}), 7.17(\mathrm{~m}, 2 \mathrm{H}, \mathrm{ArH}), 7.07(\mathrm{~m}, 2 \mathrm{H}, \mathrm{ArH}), 6.83(\mathrm{~d}$, $\left.2 \mathrm{H}, J_{\text {ortho }} 8.1 \mathrm{~Hz}, \mathrm{ArH}\right), 6.39$ and $6.32(2 \mathrm{~d}, 2 \mathrm{H}, J 15.9 \mathrm{~Hz}, \mathrm{CH}=\mathrm{CHCO})$, $5.47\left(\mathrm{~m}, 2 \mathrm{H}, \mathrm{H}_{3}\right.$ and $\left.\mathrm{H}_{5}\right), 3.89(\mathrm{~s}, 6 \mathrm{H}, 2 \times \mathrm{OMe}), 2.42\left(\mathrm{dd}, 1 \mathrm{H},{ }^{2} \mathrm{~J} 13.9\right.$ $\mathrm{Hz},{ }^{3}$ J $4.0 \mathrm{~Hz}, \mathrm{H}_{2}, \mathrm{H}_{4}$ or $\left.\mathrm{H}_{6}\right), 2.34\left(\mathrm{dd}, 1 \mathrm{H},{ }^{2}\right.$ J $13.4 \mathrm{~Hz},{ }^{3} \mathrm{~J} 8.2 \mathrm{~Hz}, \mathrm{H}_{2}, \mathrm{H}_{4}$ or $\left.\mathrm{H}_{6}\right), 2.00\left(\mathrm{~m}, 4 \mathrm{H}, \mathrm{H}_{2}, \mathrm{H}_{4}\right.$ or $\left.\mathrm{H}_{6}\right) ;{ }^{13} \mathrm{C} \mathrm{NMR}\left(\mathrm{CD}_{3} \mathrm{OD}, 75 \mathrm{MHz}, 297 \mathrm{~K}\right) \delta$ $(\mathrm{ppm})=177.4(\mathrm{COOH}), 170.9\left(2 \times \mathrm{C}_{\mathrm{q}}\right), 168.5$ and $168.2(2 \times O C O)$, $150.44,150.38\left(2 \times \mathrm{xC}_{\mathrm{q}}\right), 146.9$ and $146.8(2 \times \mathrm{CH}=\mathrm{CHCO}), 127.74$ and $127.68\left(2 \mathrm{xC}_{\mathrm{q}}\right), 124.1,124.0,123.9,116.4,115.8,115.5\left(6 \times \mathrm{CH}_{\mathrm{Ar}}\right)$, 111.7 and $111.6(2 \times \mathrm{CH}=\mathrm{CHCO}), 75.1\left(\mathrm{C}_{1}\right), 69.9,68.7\left(\mathrm{C}_{3}\right.$ and $\left.\mathrm{C}_{5}\right)$, 56.43 and 56.41 (2xOMe), 40.0, 38.7, $36.0\left(\mathrm{C}_{2}, \mathrm{C}_{4}, \mathrm{C}_{6}\right)$.

(3S,5S) 1-hydroxy-3,5 bis[[(2E)-3-(4-hydroxyphenyl)-1-oxo-2propen-1-yl]oxy]cyclohexanecarboxylic acid 4d. Lil (125 mg, 0.934 mmol) was added to a solution of $9 \mathbf{d}(105 \mathrm{mg}, 0.186 \mathrm{mmol})$ in EtOAc $(4.0 \mathrm{~mL})$ then the mixture was heated under reflux $24 \mathrm{~h}$. After cooling down to room temperature, the mixture was diluted with EtOAc $(25 \mathrm{~mL})$, washed with $3 \mathrm{M} \mathrm{HCl}(5 \mathrm{~mL})$ and extracted with EtOAc $(3 \times 30 \mathrm{~mL})$. The organic layer was dried over $\mathrm{MgSO}_{4}$, concentrated to give the crude (3S,5S) 1-hydroxy-3,5 bis[[(2E)-3-(4acetoxyphenyl)-1-oxo-2-propen-1-yl]oxy]cyclohexanecarboxylic acid 11d (quantitative crude yield), pure enough for characterization and used directly in the following step. $[\alpha]_{D}^{25}=-111.9$ (c 1.03, $\mathrm{CH}_{3} \mathrm{OH}$ ); IR(ATR) 3447, 3059, 2961, 1765, 1707, 1601, 1585, 1506, 1418, $1369,1312,1265,1202,1163,1136,1057,1009 \mathrm{~cm}^{-1} ;{ }^{1} \mathrm{H}$ NMR $\left(\mathrm{CDCl}_{3}, 400 \mathrm{MHz}\right): \delta(\mathrm{ppm})=7.68$ and $7.62\left(2 \mathrm{~d}, 2 \mathrm{H},{ }^{3} \mathrm{~J} 15.9 \mathrm{~Hz}\right.$, $\mathrm{CH}=\mathrm{CHCO}$ ), 7.52 (dd, $4 \mathrm{H}, J_{\text {ortho }} 8,6 \mathrm{~Hz}, J_{\text {ortho }} 6.8 \mathrm{~Hz}, \mathrm{Ar}-\mathrm{H}$ ), 7.11 (dd, $\left.4 \mathrm{H}, J_{\text {ortho }} 8,6 \mathrm{~Hz}, J_{\text {meta }} 2.0 \mathrm{~Hz}, \mathrm{Ar}-\mathrm{H}\right), 6.41$ and $6.34\left(2 \mathrm{~d}, 2 \mathrm{H},{ }^{3} \mathrm{~J} 15.9 \mathrm{~Hz}\right.$, $\mathrm{CH}=\mathrm{CHCO}), 5.49\left(\mathrm{~m}, 2 \mathrm{H}, \mathrm{H}_{3}\right.$ and $\left.\mathrm{H}_{5}\right), 2.36-2.02$ and $1.86(2 \mathrm{~m}, 11$ and $1 \mathrm{H}, \mathrm{H}_{2}, \mathrm{H}_{4}, \mathrm{H}_{6}$ and $\left.2 \times \mathrm{OCOCH}_{3}\right) ;{ }^{13} \mathrm{C} \mathrm{NMR}\left(\mathrm{CDCl}_{3}, 100 \mathrm{MHz}\right) \delta(\mathrm{ppm})=$ $177.0(\mathrm{COOH}), 169.41$ and $169.39\left(2 \times \mathrm{CCOCH}_{3}\right), 166.2,166.1$ (2xCOO) $152.4,152.3\left(2 x_{\mathrm{q}}\right), 144.9,144.4(2 \times \mathrm{CH}=\mathrm{CHCO}), 132.04$, $132.0\left(2 \times \mathrm{C}_{\mathrm{q}}\right), 129.6,129.5\left(4 \mathrm{xCH}_{\mathrm{Ar}}\right), 122.3\left(4 \mathrm{xCH}_{\mathrm{Ar}}\right), 118.0,117.9(2 \mathrm{x}$ $\mathrm{CH}=\mathrm{CHCO}), 75.2\left(\mathrm{C}_{1}\right), 69.4,66.6\left(\mathrm{C}_{3}\right.$ and $\left.\mathrm{C}_{5}\right), 39.3,36.5,34.9\left(\mathrm{C}_{2}, \mathrm{C}_{4}\right.$ and $\left.\mathrm{C}_{6}\right), 21.3\left(2 \mathrm{x} \quad \mathrm{OCOCH}_{3}\right) ; \mathrm{HRMS}-\mathrm{ESI} \mathrm{m} / \mathrm{z}[\mathrm{M}+\mathrm{Na}]^{+}$calcd for $\mathrm{C}_{29} \mathrm{H}_{28} \mathrm{NaO}_{11}$ 575.1524; found 575.1503.

To a solution of $(3 \mathrm{~S}, 5 \mathrm{~S})$ 1-hydroxy-3,5 bis[[(2E)-3-(4-acetoxyphenyl)1-oxo-2-propen-1-yl]oxy]cyclohexanecarboxylic acid 11d (196 mg) in acetone $(4.0 \mathrm{~mL})$ was added $3 \mathrm{M} \mathrm{HCl}$ aqueous solution $(2.0 \mathrm{~mL})$ and the mixture was refluxed for $5 \mathrm{~h}$. After completion, the reaction mixture was cooled down to room temperature, diluted with EtOAc $(30 \mathrm{~mL})$ and washed with brine $(20 \mathrm{~mL})$. The organic layer was concentrated, dissolved in $\mathrm{MeOH} / \mathrm{H}_{2} \mathrm{O}(20 / 50 \mathrm{~mL})$ and washed with pentane $(2 \times 15 \mathrm{~mL})$. After concentration, the crude was obtained quantitatively as a pale solid $4 \mathbf{d}$ (165 mg). $[\alpha]_{D}^{25}=-72.6$ (c 0.71, $\mathrm{CH}_{3} \mathrm{OH}$ ); IR (ATR) 3406, 2976, 2947, 1692, 1593, 1514, 1456, 1429, $1375,1269,1209,1177,1161,1138,1032 \mathrm{~cm}^{-1} ;{ }^{1} \mathrm{H}$ NMR $\left(\mathrm{CD}_{3} \mathrm{OD}\right.$, $400 \mathrm{MHz}, 295 \mathrm{~K}) \delta(\mathrm{ppm})=7.59$ and $7.54(2 \mathrm{~d}, 2 \mathrm{H}, J 16.0 \mathrm{~Hz}$, $\mathrm{CH}=\mathrm{CHCO}), 7.08(\mathrm{~m}, 4 \mathrm{H}, \mathrm{ArH}), 6.97-6.92(\mathrm{~m}, 4 \mathrm{H}, \mathrm{ArH}), 6.81(\mathrm{~m}, 2 \mathrm{H}$, $\operatorname{ArH}), 6.29$ and $6.23(2 \mathrm{~d}, 2 \mathrm{H}, J 16.0 \mathrm{~Hz}, \mathrm{CH}=\mathrm{CHCO}), 5.44\left(\mathrm{~m}, 2 \mathrm{H}, \mathrm{H}_{3}\right.$ and $\mathrm{H}_{5}$ ), 2.40 (dd, $1 \mathrm{H},{ }^{2} \mathrm{~J} 13.9 \mathrm{~Hz},{ }^{3} \mathrm{~J} 4.3 \mathrm{~Hz}, \mathrm{H}_{2}, \mathrm{H}_{4}$ or $\mathrm{H}_{6}$ ), 2.32 (dd, $1 \mathrm{H},{ }^{2}$ J $13.4 \mathrm{~Hz},{ }^{3}$ J $8.2 \mathrm{~Hz}, \mathrm{H}_{2}, \mathrm{H}_{4}$ or $\left.\mathrm{H}_{6}\right), 1.96\left(\mathrm{~m}, 4 \mathrm{H}, \mathrm{H}_{2}, \mathrm{H}_{4}\right.$ or $\left.\mathrm{H}_{6}\right) ;{ }^{13} \mathrm{C}$ NMR $\left(\mathrm{CD}_{3} \mathrm{OD}, 100 \mathrm{MHz}, 297 \mathrm{~K}\right) \delta(\mathrm{ppm})=177.4(\mathrm{COOH}), 171.0$ $\left(2 \times C_{q}\right), 168.6$ and 168.5 (2xOCO), 149.4, $149.3\left(2 \times C_{q}\right), 147.1$ and $146.9(2 \times \underline{C H}=\mathrm{CHCO}), 127.74$ and $127.68\left(2 \times \mathrm{C}_{\mathrm{q}}\right), 123.1,123.0,116.5$, 116.4 (x2), 115.4, 115.2, 115.11, 115.09, $115.0\left(8 \mathrm{x} \mathrm{CH}_{\mathrm{Ar}}+2 \mathrm{x}\right.$ $\mathrm{CH}=\underline{\mathrm{CHCO}}), 75.0\left(\mathrm{C}_{1}\right), 69.9,68.7\left(\mathrm{C}_{3}\right.$ and $\left.\mathrm{C}_{5}\right), 39.9,38.6,35.9\left(\mathrm{C}_{2}, \mathrm{C}_{4}\right.$, $\left.\mathrm{C}_{6}\right)$. 


\section{Conclusions}

In conclusion, new analogs of 3,5-dicaffeoyl quinic acid $\mathbf{3}$ were prepared from the previously unreported 4-deoxy triol intermediate 6. Despite reported conditions for the synthesis of natural substrates, an optimization study was required to identify new conditions leading to an efficient and repeatable synthesis of seven new dicinnamoyl conjugates. Final deprotection steps were also investigated to provide robust access to both carboxylic acid and methyl ester series. Eight compounds were evaluated in toxicity bioassays against aphids. Two compounds turned out to be growth inhibitors at lower concentrations than the natural compound, when combining both the carboxylic acid function on a pseudo quinic core and either a 3,4-dihydroxyl substitution on the two cinnamic moieties or more unexpectedly a single $p$-hydroxyl group. Moreover, the amounts ingested during our assay per insect ( $1 \mu \mathrm{l} /$ larval life to $1 \mathrm{mg}$ aphid) correspond to $250 \mathrm{ng}$, and concentrations of chlorogenic acid, the biosynthetic precursor of DiCQA, was found to be about $10 \mathrm{mM}$ in peach-tree leaves for example, ${ }^{64}$ therefore significantly higher than the active $500 \mu \mathrm{M}$ doses illustrated here. As perspectives to this work, more specific bioassays, such as behaviour-oriented shortterm bioassays, ${ }^{27}$ may be realized. Confirming the activity of the synthesized compounds on other aphid species of agronomic importance could also be performed. Moreover this work paves the way to more extensive structure-activity investigations using heterodiesters or other series of adducts.

\section{Acknowledgements}

We gratefully acknowledge INSA-Lyon for financial support (BQR 2011) and the China Scolarship Council for a PhD grant to X.L. We would also like to thank Marie-Noëlle Corre for her help in preparing 3,5dicaffeoylquinic acid Catherine Sivignon for her help in running the bioassays.

\section{Notes and references}

${ }_{2}^{1}$ IUPAC Nomenclature of cyclitols. Biochem. J. 1976, 153 ,23-31

2 As underlined by G. F. Pauli, U. Kuczkowiak and A. Nahrstedt, Magn. Reson. Chem., 1999, 37, 827 : One may be particularly careful with some representations in the literature misdrawing the structures as from the L-(+) quinic acid pool instead of the D-(-) quinic acid. The wrong numbering between positions 3 and 5 led to wrongly identidified compounds.

${ }^{3}$ M. N. Clifford, S. Knight, B. Surucu and N. Kuhnert, J. Agr. Food Chem., 2006, 54, 1957.

${ }^{4}$ R. Jaiswal, M. A. Patras, P. J. Eravuchira and N. Kuhnert, J. Agric. Food Chem., 2010, 58, 8722 .

${ }^{5}$ M. N. Clifford, J. Sc. Food Agric., 2000, 80, 1033

${ }_{7}^{6}$ R. Upadhyay and L. J. M. Rao, Critical Rev. Food Sci. Nutr., 2013, 53, 968.

${ }^{7}$ H.-P. Song, J. Chen, J.-Y. Hong, H. Hao, L.-W. Qi, J. Lu, Y. Fu, B. Wu, H. Yang and P. Li, Chem. Commun., 2015, 51, 1494.

${ }^{8}$ M. Villarino, P. Sandin-Espana, P. Melgarejo and A. De Cal, J. Agr. Food Chem., 2011, 59, 3205
${ }^{9}$ L. Fan, Y. Wang, N. Liang, X.-J. Huang, M.-M. Li, C.-L. Fan, Z.-L. Wu, Y.-L. Li and W.-C. Ye, Planta Med., 2013, 79, 1558

${ }^{10}$ R. Domitrovic, O. Cvijanovic, V. Susnic and N. Katalinic, Toxicology, 2014, 324, 98 .

${ }^{11}$ Y. Miyamae, M. Kurisu, K. Murakami, J. Han, H. Isoda, K. Irie and H. Shigemori, Bioorg. Med. Chem., 2012, 20, 5844.

12 J. Deng, X.-L. Qi, Z.-Z. Guan, X.-M. Yan, Y. Huang and Y.-L. Wang, J. Pharm. Pharmacol., 2013, 65, 1736.

${ }^{13}$ C. Wu, H. Luan, X. Zhang, S. Wang, X. Zhang, X. Sun and P. Guo, Plos One, 2014, 9.

${ }^{14}$ H. Hemmerle, H. J. Burger, P. Below, G. Schubert, R. Rippel, P. W. Schindler, E. Paulus and A. W. Herling, J. Med. Chem., 1997, 40, 137.

${ }^{15}$ I. W. G. Forbes and W. Forbes, WO2015044649A1, 2015.

${ }^{16}$ G. Pressi, A. Minghetti, N. Crespi Perellino, S. Boninsegna and R. Dal Toso, EP2133323A1, 2009.

${ }^{17}$ C. M. Smith and W.-P. Chuang, Pest Manage. Sci., 2014, 70, 528.

${ }^{18}$ H. F. Harrison, Jr., T. R. Mitchell, J. K. Peterson, W. P. Wechter, G. F. Majetich and M. E. Snook, J. Am. Soc. Horticultural. Sci., 2008, 133, 492.

${ }^{19}$ R. A. Cole, Ann. Appl. Biol., 1985, 106, 211

${ }^{20}$ M. I. Mhlongo, L. A. Piater, P. A. Steenkamp, N. E. Madala and I. A. Dubery, Physiol. Mol. Plant Pathology, 2014, 88, 61.

${ }^{21}$ R. A. Cole, Ann. Appl. Biol., 1984, 105, 129.

22 K. A. Leiss, F. Maltese, Y. H. Choi, R. Verpoorte and P. G. L. Klinkhamer, Plant Physiol., 2009, 150, 1567.

${ }^{23}$ M.-H. Sauge, J.-L. Poëssel, T. Guillemaud and L. Lapchin, ArthropodPlant Interactions, $2011, \mathbf{5}, 369$.

${ }^{24}$ M. H. Sauge, J. P. Lacroze, J. L. Poëssel, T. Pascal and J. Kervella, Entomol. Exp. Appl., 2002, 102, 29-37.

${ }^{25}$ Y. Rahbé, B. Delobel, G. Febvay and G. Massonie, Ann. Soc. Entomol. Fr., 1988, 24, 472.

${ }^{26}$ M. Kodoma, H. Wada, H. Otani, K. Kohmoto and Y. Kimura, Phytochem., 1998, 47, 371.

27 J. L. Poëssel, M. H. Collet and Y. N. E. Rahbe, WO2009095624A2, 2009.

${ }^{28}$ F. Antognoni, N. C. Perellino, S. Crippa, R. Dal Toso, B. Danieli, A. Minghetti, F. Poli and G. Pressi, Fitoterapia, 2011, 82, 950.

${ }^{29}$ S. Deshpande, R. Jaiswal, M. F. Matei and N. Kuhnert, J. Agr. Food Chem., 2014, 62, 9160.

${ }^{30}$ C. Menozzi Smarrito, C. Munari, F. Robert and D. Barron, Org. Biomol. Chem., 2008, 6, 986.

${ }^{31}$ K. S. Raheem, N. P. Botting, G. Williamson and D. Barron, Tetrahedron Lett., 2011, 52, 7175.

${ }^{32}$ V. Sinisi, C. Forzato, N. Cefarin, L. Navarini and F. Berti, Food Chem. $2015, \mathbf{1 6 8}, 332$

${ }^{33}$ V. Sinisi, K. Boronova, S. Colomban, L. Navarini, F. Berti and C. Forzato, Eur. J. Org. Chem., 2014, 1321.

${ }^{34}$ S. Blumberg, O. Frank and T. Hofmann, J. Agr. Food. Chem., 2010, 58, 3720 .

${ }^{35}$ T. de Paulis, D. E. Schmidt, A. K. Bruchey, M. T. Kirby, M. P. McDonald, P. Commers, D. M. Lovinger and P. R. Martin, Eur. J. Pharmacol., $2002,442,215$.

${ }^{36}$ K.-I. Oyama, N. Watanabe, T. Yamada, M. Suzuki, Y. Sekiguchi, T. Kondo and K. Yoshida, Tetrahedron, 2015, 71, 3120.

${ }^{37}$ W. E. Zeller, J. Agric. Food Chem., 2014, 62, 1860.

${ }^{38}$ I. Dokli, L. Navarini and Z. Hamersak, Tetrahedron: Asymmetry, 2013, 24, 785.

${ }^{39}$ T. Kondo, Y. Toyama-Kato and K. Yoshida, Tetrahedron Lett., 2005, 46, 6645.

${ }^{40}$ M. Sefkow, A. Kelling and U. Schilde, Eur. J. Org. Chem., 2001, 2735.

${ }^{41}$ M. Sefkow, Eur. J. Org. Chem., 2001, 1137.

42 Y. Miyamae, M. Kurisu, J. Han, H. Isoda and H. Shigemori, Chem. Pharm. Bull., 2011, 59.

${ }^{43}$ K. M. Brummond and J. E. DeForrest, Synlett, 2009, 1517.

${ }^{44}$ T. Nagaoka, A. H. Banskota, Q. Xiong, Y. Tezuka and S. Kadota, J. Trad. Med., 2001, 18, 183.

${ }^{45}$ R. Jaiswal, M. H. Dickman and N. Kuhnert, Org. Biomol. Chem., 2012, 10, 5266.

${ }^{46}$ W. E. Zeller, Synth. Commun., 2013, 43, 1345. 
${ }^{47}$ L. E. Tavera-Mendoza, T. D. Quach, B. Dabbas, J. Hudon, X. Liao, A. Palijan, J. L. Gleason and J. H. White, Proc. Nat. Acad. Sci. U.S.A., $2008, \mathbf{1 0 5}, 8250$.

${ }^{48}$ A. Yoshida, K. Ono, Y. Suhara, N. Saito, H. Takayama and A. Kittaka, Synlett, 2003, 1175.

49 D. Oves, M. Diaz, S. Fernandez, M. Ferrero and V. Gotor, Synth. Commun., 2001, 31, 2335.

${ }^{50}$ K. L. Perlman, R. E. Swenson, H. E. Paaren, H. K. Schnoes and H. F. Deluca, Tetrahedron Lett., 1991, 32, 7663.

${ }^{51}$ C. Cecutti, Z. Mouloungui and A. Gaset, Bioresour. Technol., 1998, 66, 63.

${ }_{53}^{52}$ P. Patschinski, C. Zhang and H. Zipse, J. Org. Chem., 2014, 79, 8348.

${ }^{53}$ When carried out on methyl (2S,3S,4aR,6S,8R,8aR)-6,8-dihydroxy-2,3dimethoxy-2,3-dimethyloctahydro-1,4-benzodioxine-6-carboxylate (CAS $364333-18-6$ ), in DMF/Pyridine $[0.4 \mathrm{M}]$ at $45^{\circ} \mathrm{C}$ for $1 \mathrm{~h}$ with $3,4-$ diacetylcaffeoyl chloride, $86 \%$ of the corresponding 3-monocaffeate was isolated wheras only $56 \%$ was obtained following reported conditions (litt. 88\%) on the same substrate. ${ }^{40}$

${ }^{54}$ Despite better efficiency of $\mathrm{Et}_{3} \mathrm{~N}$ as auxiliary base to regenerate catalytic DMAP (M. R. Heinrich, H. S. Klisa, H. Mayr, W. Steglich and H. Zipse, Angew. Chem. Int. Ed., 2003, 42, 4826), any attempt with this base gave us more degradation and difficult analysable mixtures, probably because of competitive O-1 acylation and/or migration.(see ref 32 and 45)

${ }^{55}$ T. Narender, K. P. Reddy and G. Madhur, Synthetic Commun., 2009, 39, 1949.

${ }^{56}$ Y. M. Zhu, M. Regner, F. C. Lu, H. Kim, A. Mohammadi, T. J. Pearson and J. Ralph, RSC Adv., 2013, 3, 21964.

${ }_{58}^{57}$ E. Biron and H. Kessler, J Org Chem., 2005, 70, 5183.

${ }^{58}$ D. J. Hwang, S. N. Kim, J. H. Choi and Y. S. Lee, Bioorg. Med. Chem., 2001, 9, 1429.

${ }^{59}$ See supplementary information.

${ }^{60}$ H. E. Gottlieb, V. Kotlyar and A. Nudelman, J. Org. Chem., 1997, 62, 7512.

${ }^{61}$ G. Febvay, B ;. Delobel ; Y. Rahbé Can. J. Zool. 1988, 66, 2449.

${ }^{62}$ Y. Rahbé; G. Febvay, Entomol. Exp. Appl. 1993, 67,149.

${ }^{63}$ R. F. Helm, J. Ralph and R. D. Hatfield, Carbohydr. Res., 1992, 229, 183.

${ }^{64}$ M.-H. Sauge, I. Grechi and J.-L. Poessel, Entomol. Exp. Appl., 2010, 136, 123. 


\section{Synthesis of new dicinnamoyl 4-deoxy quinic acid and methyl ester derivatives and evaluation of the toxicity against the pea aphid Acyrthosiphon pisum}

Xiubin Li, ${ }^{a}$ Lucie Grand, ${ }^{a}$ Thomas Pouleriguen, ${ }^{a}$ Yves Queneau, ${ }^{a}$ Pedro da Silva, ${ }^{b}$ Yvan Rahbe, ${ }^{b}$ Jean-Luc Poëssel, ${ }^{\mathrm{c}}$ Sylvie Moebs-Sanchez, ${ }^{a *}$

${ }^{a}$ Université Lyon 1, INSA Lyon, ICBMS UMR 5246 CNRS, CPE-Lyon, Equipe Chimie Organique et Bioorganique, Bâtiment Jules Verne, 20 Avenue Albert Einstein, F-69621 Villeurbanne Cedex, France Tel : +33-(0)4 72438098 Fax : +33-(0)4 72438896

${ }^{b}$ INSA Lyon, INRA UMR0203 BF2I, Biologie Fonctionnelle Insectes et Interactions, Bâtiment Louis Pasteur, 20 Avenue Albert Einstein, F-69621 Villeurbanne Cedex, France Tel : +33-(0)4 72436151 , Fax : +33(0)4 72438534 .

${ }^{c}$ INRA Avignon, UR 1052, GAFL, Génétique et Amélioration des Fruits et Légumes, Domaine Saint Maurice, 67 allée des Chênes, CS 60094, 84143 Montfavet Cedex - France. Tel : +33-(0)4 327226

78, Fax : +33 (0)4 32722702 .

sylvie.moebs@insa-lyon.fr

\section{General}

Reagents and solvents were supplied by Aldrich, Acros, Lancaster, Alfa Aesar, Fluka or TCI and purchased at the highest commercial quality to be used without further purification. NMR spectra were recorded on a Bruker $300\left({ }^{1} \mathrm{H}: 300 \mathrm{MHz} ;{ }^{13} \mathrm{C}: 75 \mathrm{MHz}\right)$, Bruker $400\left({ }^{1} \mathrm{H}: 400 \mathrm{MHz} ;{ }^{13} \mathrm{C}: 100 \mathrm{MHz}\right)$, or Bruker $500\left({ }^{1} \mathrm{H}: 500 \mathrm{MHz} ;{ }^{13} \mathrm{C}\right.$ : $\left.125 \mathrm{MHz}\right)$ spectrometers, at 295-298K, using $\mathrm{CDCl}_{3}$ and $\mathrm{CD}_{3} \mathrm{OD}$ as solvents. The chemical shifts $(\delta \mathrm{ppm})$ are referenced to the solvent residual peak and coupling constants $(\mathrm{Hz})$ are reported in the standard fashion. The following abbreviations are used to explain the multiplicities: $\mathrm{s}=$ singlet, $\mathrm{d}=$ doublet, $\mathrm{t}=$ triplet, $\mathrm{q}=$ quartet, quint. = quintuplet, sext. = sextuplet, hept. $=$ heptuplet, $\mathrm{m}=$ multiplet, $\mathrm{br}=$ broad. Electrospray ionization (ESI) mass spectrometry (MS) experiments were performed on a Thermo Finnigan LCQ Advantage mass. High-resolution mass spectra (HRMS) were recorded on a Finnigan Mat 95xL mass spectrometer using electrospray. Analytical thin-layer chromatography was carried out on silica gel Merck 60 D254 (0.25 mm). Flash chromatography was performed on Merck Si 60 silica gel $(40-63 \mu \mathrm{m})$. Infra-red (IR) spectra were recorded with a IRAffinity-1 Shimadzu spectrometer using Attenuated Total Reflectance (ATRMiracle), and the wavenumbers are expressed in $\mathrm{cm}^{-1}$. Optical rotations were measured on a Perkin Elmer 241 or Jasco P1010 polarimeter with a $10 \mathrm{~cm}$ cell (concentration c expressed as g/100 $\mathrm{mL}$ ). Melting points were measured using Büchi apparatus B-540. Preparative HPLC was performed on Agilent equipment with a column Phenomenex C18: Jupiter ${ }^{\circledR} 5 \mu \mathrm{m} ; 300 \AA$, LC Column $250 \mathrm{x}$ 4.6mm, flow 1ml/min; Mobile phase 0 - 2min: $80 \% \mathrm{H}_{2} \mathrm{O}$ UHQ + TFA (A) / 20\% $\mathrm{CH}_{3} \mathrm{CN}+\mathrm{TFA}$ (B); 22min: $40 \%$ A / 60\% B; 30min: 100\% B; 33min: 80\% A/ 20\% B. Samples were dissolved in $\mathrm{MeOH}$ $(6 \mathrm{~mL})$ and $10 \mu \mathrm{g}$ were injected per sample. 
All data for chlorogenic acids or related derivatives presented in this paper use the recommended IUPAC numbering system. ${ }^{1}$ The assignment of NMR signals has been made according to the numbering shown in the general structure in the figure below. As much as possible an unambiguous designation of the corresponding atoms of the lateral chains has been preferred to a numbering using numerous «"» character or secondary indexation.
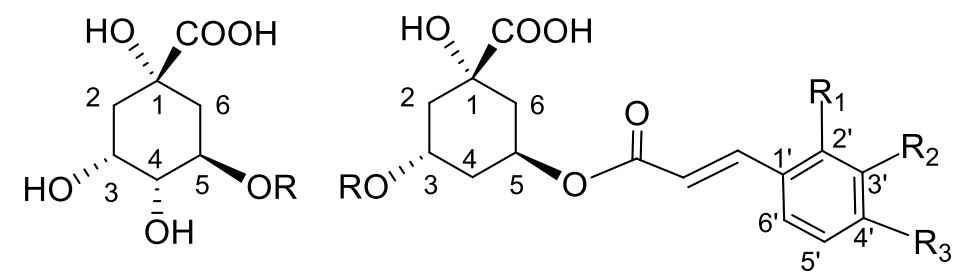

\section{List of contents}

Procedures for the synthesis of compounds 6, 9, 10, 4, 12

${ }^{1} \mathrm{H}$ and ${ }^{13} \mathrm{C}$ NMR spectra

${ }^{1} \mathrm{H}\left(\mathrm{CD}_{3} \mathrm{OD}\right)$ spectra of $\mathbf{4 a}$ and $\mathbf{4 d}$ after $6 \mathrm{~h}, 24 \mathrm{~h}, 48 \mathrm{~h}$ and $60 \mathrm{~h}$ at $296.5 \mathrm{~K}$

HPLC analysis and UV spectra for compounds 10a, 10c, 10d, 10e, 4a, 4c, 4d, 12

\footnotetext{
${ }^{1}$ IUPAC Nomenclature of cyclitols. Biochem. J. 1976 , 153 ,23-31.
} 


\section{Synthesis of compounds $(4,6,9,10,11,12)$}

\section{Methyl (3S,5S)-1,3,5-trihydroxycyclohexanecarboxylate or methyl 4-deoxy quinate 6}

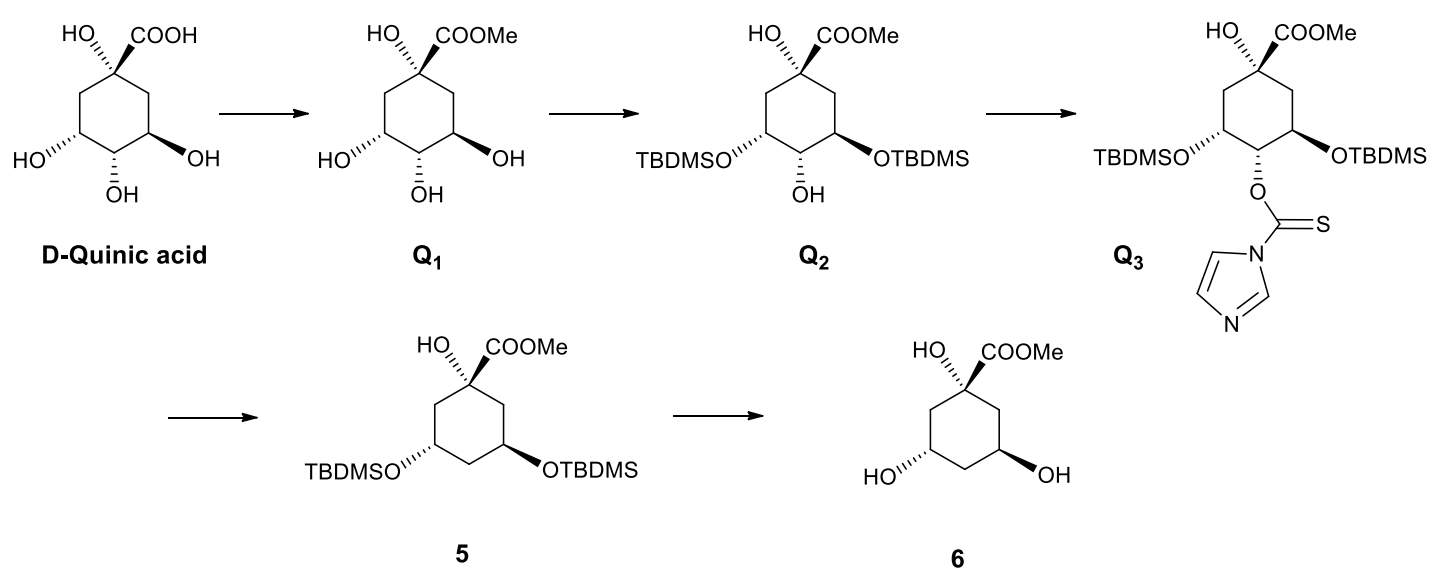

\section{Methyl (1S,3R,4S,5R)-1,3,4,5-tetrahydroxycyclohexanecarboxylate Q1 ${ }^{2}$ \\ Methyl (1S,3R,4S,5R)-1,4-dihydroxy-3,5-tert-butyldimethylsilyloxyclohexanecarboxylate $\mathbf{Q} 2^{3}$}

\section{Methyl (1S,3R,4S,5R)-1-hydroxy-4-[(1H-imidazol-1-ylcarbothioyl)oxy]-3,5-tert- butyldimethylsilyloxy cyclohexanecarboxylate $\mathbf{Q 3}^{4}$}

Q1, Q2, Q3 were prepared according reported procedures.

The disilylated compound $\mathbf{5}^{\mathbf{5}}$ (106.7 $\left.\mathrm{mg}, 0.25 \mathrm{mmol}\right)$ was stirred in $\mathrm{MeOH}(2 \mathrm{~mL})$ with Amberlyst ${ }^{\circledR} \mathrm{A} 15$ $\left(\mathrm{H}^{+}\right.$form $)$at room temperature for $24 \mathrm{~h}$. After filtration and evaporation, the crude product was purified by flash chromatography (pentane/ethyl acetate 50/50 then ethyl acetate/methanol 90/10) to provide 6 (colorless oil then white solid on standing, $42.7 \mathrm{mg}, 89 \%$ ). $[\alpha]_{D}^{25}-3.7$ (C 1.06, $\left.\mathrm{CH}_{3} \mathrm{OH}\right)$; IR(ATR) 3350, 2951, 2930, 2855, 1173, 1730, 1661, 1435, 1371, 1281, 1240, 1134, 1057, $1016 \mathrm{~cm}^{-1}$; ${ }^{1} \mathrm{H}$ NMR $\left(400 \mathrm{MHz}, 296 \mathrm{~K}, \mathrm{CD}_{3} \mathrm{OD}\right) \delta(\mathrm{ppm})=4.24-4.16\left(\mathrm{~m}, 2 \mathrm{H}, \mathrm{H}_{3}\right.$ and $\left.\mathrm{H}_{5}\right), 3.73(\mathrm{~s}, 3 \mathrm{H}, \mathrm{OMe}), 2.08(\mathrm{dd}, 1 \mathrm{H}$, ${ }^{2} J 13.8 \mathrm{~Hz},{ }^{3} \mathrm{~J}_{\mathrm{H}_{5} / \mathrm{H}_{6}} 3.3 \mathrm{~Hz}, \mathrm{H}_{6}$ or $\left.\mathrm{H}_{2}\right), 2.00\left(\mathrm{~m}, 2 \mathrm{H}, \mathrm{H}_{2}\right.$ or $\mathrm{H}_{6}$ and $\left.\mathrm{H}_{4}\right), 1.88\left(\mathrm{dd}, 1 \mathrm{H},{ }^{2} J 12.8 \mathrm{~Hz},{ }^{3} J 9.1\right.$ $\mathrm{Hz}, \mathrm{H}_{2}$ or $\left.\mathrm{H}_{6}\right), 1.77\left(\mathrm{dd}, 1 \mathrm{H},{ }^{2} J 13.8 \mathrm{~Hz},{ }^{3} J 4.8 \mathrm{~Hz}, \mathrm{H}_{6}\right.$ or $\left.\mathrm{H}_{2}\right), 1.60$ (ddd, $1 \mathrm{H},{ }^{2} J 12.7 \mathrm{~Hz},{ }^{3} J_{\mathrm{H}_{4 a}} / \mathrm{H}_{5 \mathrm{a}}$ ou $3 \mathrm{a}$ $\left.9.2 \mathrm{~Hz},{ }^{3} J_{\mathrm{H}_{4 \mathrm{a}} / \mathrm{H}_{3 \mathrm{e}} \text { ou } \mathrm{H} 5 \mathrm{e}} 3.0 \mathrm{~Hz}, \mathrm{H}_{4 \mathrm{a}}\right) ;{ }^{13} \mathrm{C} \mathrm{NMR}\left(\mathrm{CD}_{3} \mathrm{OD}, 295 \mathrm{~K}, 100 \mathrm{MHz}\right) \delta(\mathrm{ppm}) 176.1(\mathrm{COOMe})$,

\footnotetext{
${ }^{2}$ M. Frank and R. Miethchen, Carbohydr. Res. 1998, 313, 49-53

${ }^{3}$ L. Sanchez-Abella, S. Fernandez, N. Armesto, M. Ferrero and V. Gotor, J. Org. Chem. 2006, 71, 5396-5399.

${ }^{4}$ D. Oves, M. Diaz, S. Fernandez, M. Ferrero and V. Gotor, Synth. Commun., 2001, 31, 2335.

${ }^{5}$ K. L. Perlman, R. E. Swenson, H. E. Paaren, H. K. Schnoes and H. F. Deluca, Tetrahedron Lett., 1991, $32,7663$.
} 
$76.8\left(\mathrm{C}_{1}\right), 67.8\left(\mathrm{C}_{5}\right.$ or $\left.\mathrm{C}_{3}\right), 64.0\left(\mathrm{C}_{3}\right.$ or $\left.\mathrm{C}_{5}\right), 52.8(\mathrm{OMe}), 43.8\left(\mathrm{C}_{2}\right.$ or $\left.\mathrm{C}_{6}\right), 42.0\left(\mathrm{C}_{4}\right), 40.3\left(\mathrm{C}_{6}\right.$ or $\left.\mathrm{C}_{2}\right)$. MS$\mathrm{ESI} \mathrm{m} / \mathrm{z}[\mathrm{M}+\mathrm{Na}]^{+}$213.1; HRMS-ESI m/z [M+Na] $]^{+}$calcd for $\mathrm{C}_{8} \mathrm{H}_{14} \mathrm{NaO}_{5}$ 213.0733; found 213.0733.

\section{General procedure for the synthesis of homodiesters of deoxy methyl quinate 9}

\section{Procedure A :}

To a solution of triol 6 in DMF / pyridine $(\mathrm{v} / \mathrm{v} 3 / 2, \mathrm{c}=0.4 \mathrm{M})$ under nitrogen was added the protected cinnamoyl chloride (3 equiv.) The mixture was stirred at $45^{\circ} \mathrm{C}$ until completion, in general after $1 \mathrm{~h}$. Methanol was added and after $15 \mathrm{~min}$ under stirring, the resulting mixture was concentrated, diluted with EtOAc, washed with $1 \mathrm{M} \mathrm{HCl}$ then with a saturated $\mathrm{NaHCO}_{3}$ solution then with brine. The organic layer was dried over $\mathrm{Na}_{2} \mathrm{SO}_{4}$, concentrated and purified by flash chromatography on silica using the appropriate eluent.

\section{Procedure B :}

To a solution of triol 6 in DMF / pyridine $(\mathrm{v} / \mathrm{v} 3 / 2, \mathrm{c}=0.4 \mathrm{M})$ under nitrogen was added the protected cinnamoyl chloride ( 3 equiv.) The mixture was stirred at $0^{\circ} \mathrm{C}$ until completion, in general after $4 \mathrm{~h}$. Methanol was added and after $15 \mathrm{~min}$ under stirring, the resulting mixture was concentrated, diluted with EtOAc, washed with $1 \mathrm{M} \mathrm{HCl}$ then with a saturated $\mathrm{NaHCO}_{3}$ solution then with brine. The organic layer was dried over $\mathrm{Na}_{2} \mathrm{SO}_{4}$, concentrated and purified by flash chromatography on silica using the appropriate eluent.

\section{Procedure C :}

To a solution of triol 6 in dichloromethane / pyridine (v/v 5/1, c = 0.1M) under nitrogen was added the protected cinnamoyl chloride (3 equiv.) and DMAP $(20 \mathrm{~mol} \%)$. The mixture was stirred at $-18^{\circ} \mathrm{C}$ to room temperature until completion, in general after $4 \mathrm{~h}$. Methanol was added and after $15 \mathrm{~min}$ under stirring, the resulting mixture was concentrated, diluted with EtOAc, washed with $1 \mathrm{M} \mathrm{HCl}$ then with a saturated $\mathrm{NaHCO}_{3}$ solution then with brine. The organic layer was dried over $\mathrm{Na}_{2} \mathrm{SO}_{4}$, concentrated and purified by flash chromatography on silica using the appropriate eluent.

\section{Methyl (3S,5S) 1-hydroxy-3,5 bis[[(2E)-3-(3,4-diacetoxyphenyl)-1-oxo-2-propen-1- yl]oxy]cyclohexanecarboxylate 9a}

Synthesized according to the general procedure B using diacetylated caffeoyl chloride ${ }^{6}$ and methyl 4deoxy quinate 6 (105 mg, $0.553 \mathrm{mmol})$. 9a was obtained as a white solid (348 $\mathrm{mg}$, yield: 92\%) after purification by flash chromatography on silica using $\mathrm{DCM}^{-\mathrm{Et}_{2} \mathrm{O}} 12 / 1$ to $6 / 1$.

$[\alpha]_{D}^{25}=-46.9\left(\mathrm{c} 1.215, \mathrm{CH}_{2} \mathrm{Cl}_{2}\right) ;$ m.p. $=92.8-94.1^{\circ} \mathrm{C}\left(\mathrm{CH}_{2} \mathrm{Cl}_{2}\right)$; IR(ATR) 2943, 2832, 1775, 1742, $1713,1639,1504,1449,1427,1371,1321,1242,1205,1180,1147,1132,1111,1022 \mathrm{~cm}^{-1} ;{ }^{1} \mathrm{H}$ NMR $\left(\mathrm{CDCl}_{3}, 298 \mathrm{~K}, 500 \mathrm{MHz}\right) \delta(\mathrm{ppm})=7.63$ and $7.58\left(2 \mathrm{~d}, 2 \mathrm{H},{ }^{3} J 15.9 \mathrm{~Hz}, \mathrm{CH}=\mathrm{CHCO}\right), 7.39$ and 7.38 (2dd, $2 \mathrm{H}, J_{\text {ortho }} 8.4 \mathrm{~Hz}, J_{\text {meta }} 1.9 \mathrm{~Hz}, 2 \mathrm{xH}_{6}$ ), 7.35 and $7.34\left(2 \mathrm{~d}, 2 \mathrm{H}, J_{\text {meta }} 1.9 \mathrm{~Hz}, 2 \mathrm{xH}_{2}\right.$ ), $7.20(\mathrm{~d}, 2 \mathrm{H}$, $J_{\text {ortho }} 8.4 \mathrm{~Hz}, 2 \mathrm{xH}_{5}$ ) $), 6.40$ and $6.32\left(2 \mathrm{~d}, 2 \mathrm{H},{ }^{3} J 15.9 \mathrm{~Hz}, \mathrm{CH}=\mathrm{CHCO}\right), 5.51$ (ddd, $1 \mathrm{H},{ }^{2} J 13.8 \mathrm{~Hz},{ }^{3} J 9.8$

6 M. Sefkow, Eur. J. Org. Chem. 2001, 1137-1141. 
$\mathrm{Hz},{ }^{3} J 3.9 \mathrm{~Hz}, \mathrm{H}_{3}$ ou $\left.5 \mathrm{ax}\right), 5.46$ (ddd, $1 \mathrm{H},{ }^{3} J 7.8 \mathrm{~Hz},{ }^{3} J 3.9 \mathrm{~Hz}, \mathrm{H}_{3 \mathrm{e}}$ ou $\left.5 \mathrm{e}\right), 3.76\left(\mathrm{~s}, 1 \mathrm{H}, \mathrm{OH}_{1}\right), 3.74$ (s, 3H, COOMe), 2.28, 2.271 and $2.268\left(3 \mathrm{~s}, 12 \mathrm{H}, \mathrm{OCOCH}_{3}\right), 2.32\left(\mathrm{~m}, 1 \mathrm{H}, \mathrm{H}_{4}\right), 2.23-2.18\left(\mathrm{~m}, 2 \mathrm{H}, \mathrm{H}_{2}\right.$ and $\left.\mathrm{H}_{6}\right), 2.08-2.00\left(\mathrm{~m}, 2 \mathrm{H}, \mathrm{H}_{2}\right.$ and $\left.\mathrm{H}_{6}\right), 1.87$ (ddd, $\left.1 \mathrm{H},{ }^{2} J 13.6 \mathrm{~Hz},{ }^{3} \mathrm{~J} 10.3 \mathrm{~Hz},{ }^{3} J 3.45 \mathrm{~Hz}, \mathrm{H}_{4 \mathrm{a}}\right) ;{ }^{13} \mathrm{C}$ NMR $\left(\mathrm{CDCl}_{3}, 298 \mathrm{~K}, 125 \mathrm{MHz}\right) \delta(\mathrm{ppm})=175.0,168.1(2)$ and $168.0(2)\left(4 \mathrm{xOCOCH}_{3}\right), 165.8$ and 165.5 (2xCOO), 143.59 and $143.57\left(\mathrm{C}_{\mathrm{q}}\right), 143.3$ and $143.2(\underline{\mathrm{CH}}=\mathrm{CHCO}), 142.5$ and $142.4\left(\mathrm{C}_{\mathrm{q}}\right), 133.2$ and 133.1 $\left(\mathrm{C}_{\mathrm{q}}\right), 126.5$ and $126.4\left(\mathrm{C}_{6}\right), 124.0$ and $123.9\left(\mathrm{C}_{5},\right), 122.9$ and $122.8\left(\mathrm{C}_{2}\right), 119.4$ and 119.2 $(\mathrm{CH}=\underline{\mathrm{CHCO}}), 74.7\left(\mathrm{C}_{1}\right), 68.9\left(\mathrm{C}_{3}\right.$ ou $\left.\mathrm{C}_{5}\right), 66.9\left(\mathrm{C}_{5}\right.$ ou $\left.\mathrm{C}_{3}\right), 53.1(\mathrm{OMe}), 39.7\left(\mathrm{C}_{2}\right.$ ou $\left.\mathrm{C}_{6}\right), 36.7\left(\mathrm{C}_{2}\right.$ ou $\left.\mathrm{C}_{6}\right), 35.0\left(\mathrm{C}_{4}\right), 20.7(2), 20.62$ and $20.60\left(4 \mathrm{x} \quad \mathrm{OCOCH}_{3}\right) ; \mathrm{HRMS}-\mathrm{ESI} \mathrm{m} / \mathrm{z}[\mathrm{M}+\mathrm{Na}]^{+}$calcd for $\mathrm{C}_{34} \mathrm{H}_{34} \mathrm{NaO}_{15}$ 705.1790; found 705.1780.

\section{Methyl (3S,5S) 1-hydroxy-3,5 bis[[(2E)-3-(3,4-di-( prop-2-en-1-yloxy)phenyl)-1-oxo-2-propen-1- yl]oxy]cyclohexanecarboxylate $9 \mathrm{~b}$}

Synthesized according to the general procedure A using diallylated caffeoyl chloride. ${ }^{7}$. and 6 (102 mg, $0.537 \mathrm{mmol}$ ) Purification by flash chromatography on silica using petroleum ether/EtOAc 50/50, providing the triester $(51 \mathrm{mg}, 10 \%)$ and diester $\mathbf{9 b}(214 \mathrm{mg}, 59 \%)$ as white solids.

$[\alpha]_{D}^{25}-140.8\left(C 0.86, \mathrm{CH}_{2} \mathrm{Cl}_{2}\right)$; $[\alpha]_{D}^{23}$ m.p. $89-91^{\circ} \mathrm{C}$; IR(ATR) 2922, 2827, 1706, 1631, 1597, 1510,

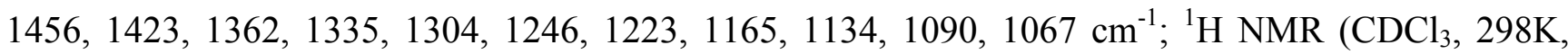
$300 \mathrm{MHz}) \delta(\mathrm{ppm})=7.63$ and $7.58\left(2 \mathrm{~d}, 2 \mathrm{H},{ }^{3} \mathrm{~J} 15.9 \mathrm{~Hz}, \mathrm{CH}=\mathrm{CHCO}\right), 7.08\left(\mathrm{~m}, 4 \mathrm{H}, \mathrm{CH}_{\mathrm{Ar}}\right), 6.87(\mathrm{~m}$, $\left.2 \mathrm{H}, \mathrm{CH}_{\mathrm{Ar}}\right), 6.31$ and $6.23\left(2 \mathrm{~d}, 2 \mathrm{H},{ }^{3} \mathrm{~J} 15.9 \mathrm{~Hz}, \mathrm{CH}=\mathrm{CHCO}\right), 6.07\left(\mathrm{~m}, 4 \mathrm{H}, \mathrm{CH}_{2}=\mathrm{CHCH}_{2} \mathrm{O}\right), 5.60-5.50$ $\left(\mathrm{m}, 2 \mathrm{H}, \mathrm{H}_{3}\right.$ and $\left.\mathrm{H}_{5}\right), 5.42-5.39\left(\mathrm{~m}, 2 \mathrm{H}, \mathrm{CH}_{2}\right.$ anti= $\left.\mathrm{CHCH}_{2} \mathrm{O}\right), 5.30\left(\mathrm{~m}, 4 \mathrm{H}, \mathrm{CH}_{2} \mathrm{syn}=\mathrm{CHCH}_{2} \mathrm{O}\right), 4.64$ (m, $8 \mathrm{H}, \mathrm{CH}_{2}=\mathrm{CHCH}_{2} \mathrm{O}$ ), $2.32\left(\mathrm{~m}, 1 \mathrm{H}, \mathrm{H}_{2}\right.$ or $\mathrm{H}_{4}$ or $\left.\mathrm{H}_{6}\right), 2.24-2.02\left(\mathrm{~m}, 4 \mathrm{H}, \mathrm{H}_{2}\right.$ or $\mathrm{H}_{4}$ or $\left.\mathrm{H}_{6}\right), 1.86$ (ddd, $1 \mathrm{H}, J 13.7 \mathrm{~Hz}, J 10.4 \mathrm{~Hz}, J 3.4 \mathrm{~Hz}, \mathrm{H}_{2}$ or $\mathrm{H}_{4}$ or $\left.\mathrm{H}_{6}\right) ;{ }^{13} \mathrm{C} \mathrm{NMR}\left(\mathrm{CDCl}_{3}, 296 \mathrm{~K}, 75 \mathrm{MHz}\right) \delta(\mathrm{ppm})=$ 175.1 (COOMe), 166.5 and $166.3(\mathrm{OCO}), 150.8\left(2 \mathrm{xC}_{\mathrm{q}}\right), 148.7\left(2 \mathrm{xC}_{\mathrm{q}}\right), 145.4$ and $145.1(\underline{\mathrm{CH}}=\mathrm{CHCO})$, $133.2133 .1,133.05$ and $133.01\left(4 \mathrm{xCH}_{2}=\mathrm{CHCH}_{2} \mathrm{O}\right), 127.64,127.57(2 \mathrm{xCq}), 123.2$ and 122.9 (2xCHAr), 118.15, 118.11, 118.1, $118.07\left(4 \mathrm{xCH}_{2}=\mathrm{CHCH}_{2} \mathrm{O}\right), 115.88$ and $115.84(\mathrm{CH}=\mathrm{CHCO})$, 113.50, 113.44, 112.7 and $112.5\left(4 \mathrm{xCH}_{\mathrm{Ar}}\right), 75.0\left(\mathrm{C}_{1}\right), 70.1,70.0$, and 69.8(2) $\left(4 \mathrm{xCH}_{2}=\mathrm{CHCH}_{2} \mathrm{O}\right), 68.8$ and $66.6\left(\mathrm{C}_{3}\right.$ and $\left.\mathrm{C}_{5}\right), 53.2(\mathrm{OMe}), 40.0,36.9$ and $35.2\left(\mathrm{C}_{2}, \mathrm{C}_{4}\right.$ and $\left.\mathrm{C}_{6}\right)$; HRMS-ESI m/z $[\mathrm{M}+\mathrm{Na}]^{+}$calcd for $\mathrm{C}_{38} \mathrm{H}_{42} \mathrm{NaO}_{11}$ 697.2619; found 697.2615.

\section{Methyl (3S,5S) 1-hydroxy-3,5 bis[[(2E)-3-(3-acetoxy,4-methoxyphenyl)-1-oxo-2-propen-1- yl]oxy]cyclohexanecarboxylate 9c}

Synthesized according to the general procedure C using acetylated feruloyl chloride and methyl 4deoxy quinate 6 (117 mg, $0.616 \mathrm{mmol})$. 9c was obtained as a white solid (266 mg, yield: 69\%) after purification by flash chromatography on silica using $\mathrm{DCM} / \mathrm{Et}_{2} \mathrm{O} 10 / 1$.

$[\alpha]_{D}^{25}=-74.5\left(\mathrm{c} 1.05, \mathrm{CH}_{2} \mathrm{Cl}_{2}\right) ;[\alpha]_{D}^{23}$ m.p. $90-92^{\circ} \mathrm{C}$; IR(ATR) 1765, 1709, 1638, 1508, 1464, 1418, 1369, 1298, 1263, 1215, 1198, 1153, 1123, 1033, $1011 \mathrm{~cm}^{-1} ;{ }^{1} \mathrm{H}$ NMR $\left(\mathrm{CDCl}_{3}, 296 \mathrm{~K}, 300 \mathrm{MHz}\right) \delta$ $(\mathrm{ppm})=7.65$ and $7.60\left(2 \mathrm{~d}, 2 \mathrm{H},{ }^{3} J 15.9 \mathrm{~Hz}, \mathrm{CH}=\mathrm{CHCO}\right), 7.12-7.02\left(\mathrm{~m}, 6 \mathrm{H}, \mathrm{CH}_{\mathrm{Ar}}\right), 6.41$ and $6.33(2 \mathrm{~d}$, $\left.2 \mathrm{H},{ }^{3} \mathrm{~J} 15.9 \mathrm{~Hz}, \mathrm{CH}=\mathrm{CHCO}\right), 5.55$ and $5.49\left(2 \mathrm{~m},{ }^{3} \mathrm{~J} 9.3 \mathrm{~Hz},{ }^{3} J 3.3 \mathrm{~Hz}, \mathrm{H}_{5}\right.$ and $\left.\mathrm{H}_{3}\right), 3.85$ and $3.84(2 \mathrm{~s}$, $2 \times 3 \mathrm{H}, 2 \mathrm{xOMe}), 3.76(\mathrm{~s}, 3 \mathrm{H}, \mathrm{COOMe}), 2.30(\mathrm{~s}, 6 \mathrm{H}, 2 \mathrm{xOAc}), 2.28\left(\mathrm{~m}, 1 \mathrm{H}, \mathrm{H}_{4 \mathrm{e}}\right), 2.22-2.18$ and 2.11-

7 Jaiswal, R.; Dickman, M. H.; Kuhnert, N. Org. Biomol. Chem. 2012, 10, 5266-5277 
2.04(m, $2 \mathrm{x} 2 \mathrm{H}, 2 \mathrm{xH}_{6}$ and $\left.2 \mathrm{xH}_{2}\right), 1.88\left(\mathrm{ddd}, 1 \mathrm{H},{ }^{2} J 13.6 \mathrm{~Hz},{ }^{3} J 10.4 \mathrm{~Hz},{ }^{3} J 3.4 \mathrm{~Hz}, \mathrm{H}_{4}\right)$; ${ }^{13} \mathrm{C} \mathrm{NMR}$ $\left(\mathrm{CDCl}_{3}, 295 \mathrm{~K}, 100 \mathrm{MHz}\right) \delta(\mathrm{ppm})=175.0(\mathrm{COOMe}), 168.8\left(2 \mathrm{xOCOCH}_{3}\right), 166.1$ and $165.8(\mathrm{COO})$, $151.4\left(2 \mathrm{xC}_{\mathrm{q}}\right), 144.6$ and $144.4(\underline{\mathrm{CH}}=\mathrm{CHCO}), 141.54$ and $141.51\left(\mathrm{C}_{\mathrm{q}}\right), 133.34$ and $133.30\left(\mathrm{C}_{\mathrm{q}}\right), 123.33$ and 123.25(CHAr), 121.6 and 121.3 (CHAr), 118.3 and $118.2(\mathrm{CH}=\underline{\mathrm{CHCO}}), 111.3$ and $111.1\left(\mathrm{CH}_{\mathrm{Ar}}\right)$, $74.8\left(\mathrm{C}_{1}\right), 68.9\left(\mathrm{C}_{3}\right.$ or $\left.\mathrm{C}_{5}\right), 66.8\left(\mathrm{C}_{5}\right.$ or $\left.\mathrm{C}_{3}\right), 56.0(2 \mathrm{xOMe}), 53.1(\mathrm{COOMe}), 39.8$ and $36.8\left(\mathrm{C}_{6}\right.$ and $\left.\mathrm{C}_{2}\right)$, $35.1\left(\mathrm{C}_{4}\right), 20.7\left(2 \mathrm{xOCOCH}{ }_{3}\right)$; HRMS-ESI m/z [M+Na] ${ }^{+}$calcd for $\mathrm{C}_{32} \mathrm{H}_{34} \mathrm{NaO}_{13} 649$ 649.1892; found 649.1865 .

\section{Methyl (3S,5S) 1-hydroxy-3,5 bis[[(2E)-3-(4-acetoxyphenyl)-1-oxo-2-propen-1- yl]oxy]cyclohexanecarboxylate 9d}

Synthesized according to the general procedure A using acetylated para-coumaroyl chloride ${ }^{8}$ and methyl 4-deoxy quinate 6 (58mg, $0.305 \mathrm{mmol})$. 9d was obtained as a white solid (142 $\mathrm{mg}$, yield: 82\%)

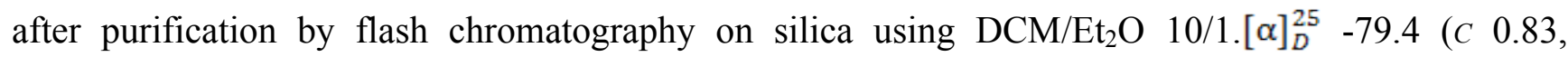
$\mathrm{CH}_{2} \mathrm{Cl}_{2}$ ); m.p. 86- $88^{\circ} \mathrm{C}$; IR(ATR) $1765,1736,1709,1638,1601,1506,1418,1369,1312,1265,1202$, $1163,1140,1086,1063,1011 \mathrm{~cm}^{-1},{ }^{1} \mathrm{H}$ NMR $\left(\mathrm{CDCl}_{3}, 295 \mathrm{~K}, 400 \mathrm{MHz}\right) \delta(\mathrm{ppm})=7.67$ and $7.62(2 \mathrm{~d}$, $\left.2 \mathrm{H},{ }^{3} J 16.0 \mathrm{~Hz}, \mathrm{CH}=\mathrm{CHCO}\right), 7.52$ and $7.50\left(2 \mathrm{~d}, 4 \mathrm{H}, J_{\text {ortho }} 8.5 \mathrm{~Hz}, \mathrm{H}_{2}\right.$ ), 7.10 (2d, $4 \mathrm{H}, J_{\text {ortho }} 8.5 \mathrm{~Hz}, \mathrm{H}_{3^{\prime}}$ ), 6.41 and $6.33\left(2 \mathrm{~d}, 2 \mathrm{H},{ }^{3} J 16.0 \mathrm{~Hz}, \mathrm{CH}=\mathrm{CHCO}\right), 5.53$ and $5.48\left(2 \mathrm{~m}, 2 \mathrm{H},{ }^{3} J 4.0 \mathrm{~Hz}, \mathrm{H}_{3}\right.$ and $\left.\mathrm{H}_{5}\right), 3.74$ (s, $3 \mathrm{H}, \mathrm{COOMe}), 2.27$ (s, 6H, OAc), $2.24\left(\mathrm{~m}, 1 \mathrm{H}, \mathrm{H}_{4}\right), 2.23-2.19\left(\mathrm{~m}, 2 \mathrm{H}, \mathrm{H}_{2}\right.$ and $\left.\mathrm{H}_{6}\right), 2.11-2.06(\mathrm{~m}, 2 \mathrm{H}$, $\mathrm{H}_{2}$ and $\left.\mathrm{H}_{6}\right), 1.88$ (ddd, $\left.1 \mathrm{H},{ }^{2} J 13.0 \mathrm{~Hz},{ }^{3} \mathrm{~J} 10.2 \mathrm{~Hz},{ }^{3} \mathrm{~J} 3.5 \mathrm{~Hz}, \mathrm{H}_{4}\right) ;{ }^{13} \mathrm{C} \mathrm{NMR}\left(\mathrm{CDCl}_{3}, 295 \mathrm{~K}, 100 \mathrm{MHz}\right)$ $\delta(\mathrm{ppm})=174.9(\underline{\mathrm{COOMe}}), 169.2\left(2 \mathrm{xOCOCH}_{3}\right), 166.1$ and $165.8(\mathrm{COO}), 152.2$ and $152.1\left(\mathrm{C}_{\mathrm{q}}\right), 144.1$ and $144.0(\underline{\mathrm{CH}}=\mathrm{CHCO}), 132.1$ and $132.0\left(2 \mathrm{xCH}_{\mathrm{Ar}}\right), 129.4$ and $129.3\left(2 \mathrm{xCH}_{\mathrm{Ar}}\right), 122.2$ and $122.1\left(\mathrm{C}_{\mathrm{q}}\right)$, 118.3 and $118.1(\mathrm{CH}=\underline{\mathrm{CHCO}}), 74.7\left(\mathrm{C}_{1}\right), 68.8$ and $66.8\left(\mathrm{C}_{3}\right.$ and $\left.\mathrm{C}_{5}\right), 53.0(\mathrm{OMe}), 39.7$ and $36.8\left(\mathrm{C}_{2}\right.$ and $\left.\mathrm{C}_{6}\right) 35.0\left(\mathrm{C}_{4}\right) 21.1\left(2 \mathrm{xCH}_{3}\right)$; HRMS-ESI m/z $[\mathrm{M}+\mathrm{Na}]^{+}$calcd for $\mathrm{C}_{30} \mathrm{H}_{30} \mathrm{NaO}_{11}$ 589.1680; found 589.1655 .

\section{Methyl (3S,5S) 1-hydroxy-3,5 bis[[(2E)-3-(2-acetoxyphenyl)-1-oxo-2-propen-1- yl]oxy]cyclohexanecarboxylate 9e}

Synthesized according to the general procedure A using acetylated ortho-coumaroyl chloride and

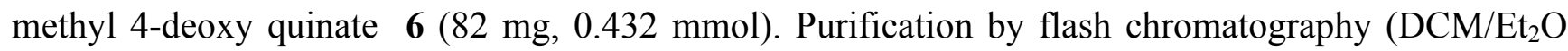
10/1) afforded 9e as a white solid (152 mg, yield: 62\%)

$[\alpha]_{D}^{25}-50.0\left(C 1.12, \mathrm{CH}_{2} \mathrm{Cl}_{2}\right)$; m.p. $87-90^{\circ} \mathrm{C}$; IR(ATR) 1763, 1711, 1638, 1483, 1454, 1437, 1371, $1315,1265,1198,1179,1140,1094,1063,1009 \mathrm{~cm}^{-1} ;{ }^{1} \mathrm{H}$ NMR $\left(\mathrm{CDCl}_{3}, 296 \mathrm{~K}, 300 \mathrm{MHz}\right) \delta(\mathrm{ppm})=$ 7.81 and $7.73(2 \mathrm{~d}, 2 \mathrm{H}, J 16.1 \mathrm{~Hz}, \mathrm{CH}=\mathrm{CHCO}), 7.63(\mathrm{dd}, 2 \mathrm{H}, J 7.9 \mathrm{~Hz}, \mathrm{H}$ ortho), 7.40 (dd, 2H, $J 7.40$ $\mathrm{Hz}, \mathrm{H}$ meta OAc ), 7.25 (dd, 2H, J 7.6 Hz, H para OAc ), 7.11 (d, 3H, J 8.1 Hz, H orthoOAc), 6.46 and $6.40(2 \mathrm{~d}, 2 \mathrm{H}, J 16.1 \mathrm{~Hz}, \mathrm{CH}=\mathrm{CHCO}), 5.53\left(\mathrm{~m}, 2 \mathrm{H}, \mathrm{H}_{3}\right.$ and $\left.\mathrm{H}_{5}\right), 3.75(\mathrm{~s}, 3 \mathrm{H}, \mathrm{COOMe}), 2.40$ and 2.38 (2s, 2x3H, 2xOAc), 2.29-2.19 (m, 3H, H-2, H-4, H6), 2.13-2.02 (m, 2H, H-2, H-6), 1.90 (ddd, 1H, ${ }^{2} \mathrm{~J}$ $\left.13.5 \mathrm{~Hz},{ }^{3} \mathrm{~J} 10.0 \mathrm{~Hz},{ }^{3} \mathrm{~J} 3.4 \mathrm{~Hz}, \mathrm{H}-4\right) ;{ }^{13} \mathrm{C} \mathrm{NMR}\left(\mathrm{CDCl}_{3}, 295 \mathrm{~K}, 100 \mathrm{MHz}\right) \delta(\mathrm{ppm})=175.0(\mathrm{COOMe})$, 169.45 and $169.40\left(\mathrm{OCOCH}_{3}\right), 165.8$ and $165.6(\mathrm{COO}), 149.4\left(2 \times \mathrm{C}_{\mathrm{q}}\right), 138.56$ and 138.50

\footnotetext{
${ }^{8}$ R. F. Helm, J. Ralph and R. D. Hatfield, Carbohydr. Res., 1992, 229, 183-194.
} 
$(\underline{\mathrm{CH}}=\mathrm{CHCO}), 131.34$ and $131.30\left(\mathrm{C}_{4}\right), 127.6$ and $127.5\left(\mathrm{C}_{6}\right), 127.1$ and $127.0\left(\mathrm{C}_{\mathrm{q}}\right), 126.44$ and 126.41( $\left(\mathrm{C}_{5},\right), 123.26$ and $123.20\left(\mathrm{C}_{3}{ }\right), 120.2$ and $120.1(\mathrm{CH}=\underline{\mathrm{CHCO}}), 74.6\left(\mathrm{C}_{1}\right), 68.8\left(\mathrm{C}_{3}\right.$ or $\left.\mathrm{C}_{5}\right), 67.0$ $\left(\mathrm{C}_{5}\right.$ or $\left.\mathrm{C}_{3}\right), 53.2(\mathrm{COOMe}), 39.7$ and $36.9\left(\mathrm{C}_{6}\right.$ and $\left.\left.\mathrm{C}_{2}\right), 35.1\left(\mathrm{C}_{4}\right), 21.0(2 \mathrm{x} \mathrm{OCOCH})_{3}\right) ; \mathrm{HRMS}-\mathrm{ESI} \mathrm{m} / \mathrm{z}$ $[\mathrm{M}+\mathrm{Na}]^{+}$calcd for $\mathrm{C}_{30} \mathrm{H}_{30} \mathrm{NaO}_{11} 589.1680$; found 589.1669 .

\section{General procedure for the deprotection of aromatic acetates}

Procedure $\mathbf{D}^{9}$ : To a solution of compound 9 (a, c, d or e) in ethanol/water $(2 / 1,0.12 \mathrm{M})$ was added hydrazine acetate $(\mathrm{NaOAc}, 20 \mathrm{eq})$. After stirring at reflux for $1 \mathrm{~h}$, the mixture was diluted with EtOAc, washed with brine then the organic layer was dried over $\mathrm{MgSO}_{4}$, concentrated and purified by flash chromatography.

Procedure $\mathbf{E}^{10}$ : To a solution of compound 9 (a, c, d or e) in $\operatorname{DMF}(0.15 \mathrm{M})$ was added hydrazine acetate (75equiv.). After stirring at room temperature for $1 \mathrm{~h}$, the mixture was diluted with EtOAc, washed with brine then the organic layer was dried over $\mathrm{MgSO}_{4}$, concentrated and purified by flash chromatography.

\section{Methyl (3S,5S) 1-hydroxy-3,5 bis[[(2E)-3-(3,4-dihydroxyphenyl)-1-oxo-2-propen-1- yl]oxy]cyclohexanecarboxylate 10a}

To a solution of 9a $(116 \mathrm{mg}, 0.170 \mathrm{mmol})$ in DMF $(0.9 \mathrm{~mL})$ was added hydrazine acetate $(71 \mathrm{mg}, 0.64$ $\mathrm{mmol})$. After stirring at room temperature for $1 \mathrm{~h}$, the mixture was diluted with EtOAc $(50 \mathrm{~mL})$, washed with brine $\left(30 \mathrm{~mL}\right.$ x 3 ) then the organic layer was dried over $\mathrm{MgSO}_{4}$, concentrated and purified by flash chromatography $\left(\mathrm{CH}_{2} \mathrm{Cl}_{2} / \mathrm{CH}_{3} \mathrm{OH} 40 / 1\right)$ to provide $\mathbf{1 0 a}$ as a colorless oil $(78 \mathrm{mg}$, $89 \%$ ). $[\alpha]_{D}^{25}-167.0$ (C 0.64, $\mathrm{CH}_{3} \mathrm{OH}$ ); IR(ATR) 3323, 3304, 3285, 3275, 2949, 2835, 1680, 1630, 1599, 1516, 1443, 1373, 1256, 1159, 1140, 1119, 1067, $1016 \mathrm{~cm}^{-1} ;{ }^{1} \mathrm{H}$ NMR $\left(\mathrm{CD}_{3} \mathrm{OD}, 298 \mathrm{~K}, 500\right.$ $\mathrm{MHz}): \delta \mathrm{ppm}=7.58$ and $7.52\left(\mathrm{~d}, 2 \mathrm{H},{ }^{3} \mathrm{~J} 15.8 \mathrm{~Hz}, \mathrm{CH}=\mathrm{CHCO}\right), 7.06\left(\mathrm{bs}, 2 \mathrm{H}, \mathrm{Ar}-\mathrm{H}_{2}\right), 6.95(\mathrm{dt}, 2 \mathrm{H}$, $\left.J_{\text {ortho }}=8.1, J_{\text {meta }} 2.3 \mathrm{~Hz}, \mathrm{Ar}-\mathrm{H}_{6}{ }^{\prime}\right), 6.80\left(\mathrm{dd}, 2 \mathrm{H}, J_{\text {ortho }} 8,2 \mathrm{~Hz}, J_{\text {meta }} 2,7 \mathrm{~Hz}, \mathrm{Ar}-\mathrm{H}_{5}{ }\right), 6.28$ and $6.19(\mathrm{~d}, 2 \mathrm{H}$, $\left.{ }^{3} J 15.9 \mathrm{~Hz}, \mathrm{CH}=\mathrm{CHCO}\right), 5.42\left(\mathrm{~m}, 2 \mathrm{H}, \mathrm{H}_{3}\right.$ and $\left.\mathrm{H}_{5}\right), 3.70(\mathrm{~s}, 3 \mathrm{H}, \mathrm{OMe}), 2.46\left(\mathrm{dd}, 1 \mathrm{H},{ }^{2} J 13.4 \mathrm{~Hz},{ }^{3} J 3.5\right.$ $\mathrm{Hz}, \mathrm{H}_{2}$ or $\left.\mathrm{H}_{6}\right), 2.39$ (dd, $1 \mathrm{H},{ }^{2} J 13.6 \mathrm{~Hz},{ }^{3} J 6.9 \mathrm{~Hz}, \mathrm{H}_{2}$ or $\left.\mathrm{H}_{6}\right), 2.06\left(\mathrm{~m}, 1 \mathrm{H}, \mathrm{H}_{4}\right), 1.97\left(\mathrm{~m}, 2 \mathrm{H}, \mathrm{H}_{4}\right.$ and $\mathrm{H}_{2}$ or $\left.\mathrm{H}_{6}\right), 1.86\left(\mathrm{dd}, 1 \mathrm{H},{ }^{2} J 13.4 \mathrm{~Hz},{ }^{3} J 7.7 \mathrm{~Hz}, \mathrm{H}_{2}\right.$ or $\left.\mathrm{H}_{6}\right) ;{ }^{13} \mathrm{C} \mathrm{NMR}\left(\mathrm{CD}_{3} \mathrm{OD}, 298 \mathrm{~K}, 125 \mathrm{MHz}\right) \delta(\mathrm{ppm})=$ 175.7 (COOMe), 168.5 (OCO), 168.0 (OCO), $149.6\left(\mathrm{C}_{\mathrm{q}}\right), 149.5\left(\mathrm{C}_{\mathrm{q}}\right), 147.2$ and $147.0(\mathrm{CH}=\mathrm{CHCO})$, $146.8\left(\mathrm{C}_{\mathrm{q}}\right), 146.7\left(\mathrm{C}_{\mathrm{q}}\right), 127.7\left(\mathrm{C}_{\mathrm{q}}\right), 127.6\left(\mathrm{C}_{\mathrm{q}}\right), 123.04$ and $123.00\left(\mathrm{C}_{6}\right), 116.53$ and $116.50\left(\mathrm{C}_{5}\right), 115.4$ and $115.1(\mathrm{OCOCH}=\underline{\mathbf{C H}}), 115.09$ and $115.00\left(\mathrm{C}_{2}\right), 75.0\left(\mathrm{C}_{1}\right), 69.5$ and $69.0\left(\mathrm{C}_{3}\right.$ and $\left.\mathrm{C}_{5}\right), 53.0(\mathrm{OMe})$, 39.7, 39.3 and $35.9\left(3 \mathrm{xCH}_{2}, \mathrm{C}_{2}, \mathrm{C}_{6}\right.$ and $\left.\mathrm{C}_{4}\right)$; HRMS-ESI $\mathrm{m} / \mathrm{z}[\mathrm{M}+\mathrm{Na}]^{+}$calcd for $\mathrm{C}_{26} \mathrm{H}_{26} \mathrm{NaO}_{11}$ 537.1367; found 537.1351.

\footnotetext{
${ }^{9}$ T. Narender, K. P. Reddy and G. Madhur, Synthetic Commun., 2009, 39, 1949-1956.

${ }^{10}$ Y. M. Zhu, M. Regner, F. C. Lu, H. Kim, A. Mohammadi, T. J. Pearson and J. Ralph, RSC Adv., $2013,3,21964$.
} 


\section{Methyl (3S,5S) 1-hydroxy-3,5 bis[[(2E)- 3-(4-hydroxy-3-methoxyphenyl)-1-oxo-2-propen-1- yl]oxy]cyclohexanecarboxylate 10c}

The general procedure $\mathrm{E}$ for the deprotection of aromatic acetates was performed with $9 \mathrm{c}(65 \mathrm{mg}$, $0.104 \mathrm{mmol})$ in DMF $(0.45 \mathrm{~mL})$ and hydrazine acetate $(43 \mathrm{mg}, 0.47 \mathrm{mmol})$ providing after purification 10c as a colorless oil (50 mg, 89\%). [ $\alpha]_{D}^{25}-170.0\left(C\right.$ 0.93, $\left.\mathrm{CH}_{3} \mathrm{OH}\right)$; IR(ATR) 3516, 3057, 2957, 2941, $1701,1631,1593,1512,1462,1454,1429,1377,1263,1207,1157,1138,1032,1009 \mathrm{~cm}^{-1}$; ${ }^{1} \mathrm{H}$ NMR $\left(\mathrm{CD}_{3} \mathrm{OD}, 400 \mathrm{MHz}\right): \delta \mathrm{ppm}=7.63$ and $7.57\left(\mathrm{~d}, 2 \mathrm{H},{ }^{3} J 15.9 \mathrm{~Hz}, \mathrm{CH}=\mathrm{CHCO}\right), 7.12$ (bs, $\left.2 \mathrm{H}, \mathrm{Ar}-\mathrm{H}\right)$, $7.07\left(\mathrm{dd}, 2 \mathrm{H}, J_{\text {ortho }} 8,2 \mathrm{~Hz}, J_{\text {meta }} 2,7 \mathrm{~Hz}, \mathrm{Ar}-\mathrm{H}\right), 6.81\left(\mathrm{dd}, 2 \mathrm{H}, J_{\text {ortho }} 8,2 \mathrm{~Hz}, J_{\text {meta }} 2,2 \mathrm{~Hz}, \mathrm{Ar}-\mathrm{H}\right), 6.38$ and $6.29\left(\mathrm{~d}, 2 \mathrm{H},{ }^{3} \mathrm{~J} 15.9 \mathrm{~Hz}, \mathrm{CH}=\mathrm{CHCO}\right), 5.432\left(\mathrm{~m}, 2 \mathrm{H}, \mathrm{H}_{3}\right.$ and $\left.\mathrm{H}_{5}\right), 3.88(\mathrm{~s}, 6 \mathrm{H}, 2 \mathrm{x} \mathrm{OMe}), 3.70(\mathrm{~s}, 3 \mathrm{H}$, COOMe), $2.45\left(\mathrm{dd}, 1 \mathrm{H},{ }^{2} J 13.6 \mathrm{~Hz},{ }^{3} J 4.1 \mathrm{~Hz}, \mathrm{H}_{2}\right.$ or $\left.\mathrm{H}_{6}\right), 2.38\left(\mathrm{dd}, 1 \mathrm{H},{ }^{2} J 13.6 \mathrm{~Hz},{ }^{3} \mathrm{~J} 7.2 \mathrm{~Hz}, \mathrm{H}_{2}\right.$ or $\left.\mathrm{H}_{6}\right), 2.14-1.97\left(\mathrm{~m}, 3 \mathrm{H}, 2 \mathrm{x} \mathrm{H} \mathrm{H}_{4}\right.$ and $\mathrm{H}_{2}$ or $\left.\mathrm{H}_{6}\right), 1.88\left(\mathrm{dd}, 1 \mathrm{H},{ }^{2} J 13.6 \mathrm{~Hz},{ }^{3} J 7.5 \mathrm{~Hz}, \mathrm{H}_{2}\right.$ or $\left.\mathrm{H}_{6}\right) ;{ }^{13} \mathrm{C} \mathrm{NMR}$ $\left(\mathrm{CD}_{3} \mathrm{OD}, 100 \mathrm{MHz}\right) \delta(\mathrm{ppm})=175.7((\mathrm{COOMe}), 168.4$ and $168.0(\mathrm{OCO}), 150.7,150.6,149.4,149.3$ $\left(\mathrm{C}_{\mathrm{q} \mathrm{Ar}}\right), 147.1$ and $147.0(\underline{\mathrm{CH}}=\mathrm{CHCO}), 127.7$ and $127.5\left(\mathrm{C}_{\mathrm{q} \mathrm{Ar}}\right), 124.2$ and $124.1\left(\mathrm{CH}_{\mathrm{Ar}}\right), 116.5$ and $116.4\left(\mathrm{CH}_{\mathrm{Ar}}\right), 115.7$ and $115.4(\mathrm{OCOCH}=\underline{\mathbf{C H}}), 111.6\left(\mathrm{CH}_{\mathrm{Ar}}\right), 75.0\left(\mathrm{C}_{1}\right), 69.5$ and $69.0\left(\mathrm{C}_{5}\right.$ or $\left.\mathrm{C}_{3}\right)$, 56.4(2) $\left(2 \mathrm{xOCH}_{3}\right), 53.0(\mathrm{COOMe}), 39.8$ and $39.2\left(\mathrm{C}_{2}\right.$ or $\left.\mathrm{C}_{6}\right), 35.9\left(\mathrm{C}_{4}\right) ; \mathrm{HRMS}-\mathrm{ESI} \mathrm{m} / \mathrm{z}[\mathrm{M}+\mathrm{Na}]^{+}$ calcd for $\mathrm{C}_{28} \mathrm{H}_{30} \mathrm{NaO}_{11} 65.1680$; found 565.1662.

\section{Methyl (3S,5S) 1-hydroxy-3,5 bis[[(2E)-3-(4-hydroxyphenyl)-1-oxo-2-propen-1- yl]oxy]cyclohexanecarboxylate 10d}

The general procedure $\mathrm{E}$ for the deprotection of aromatic acetates was performed with $9 \mathbf{d}(80 \mathrm{mg}, 0.14$ $\mathrm{mmol})$ in DMF $(0.6 \mathrm{~mL})$ and hydrazine acetate $(59 \mathrm{mg}, 0.64 \mathrm{mmol})$ providing after purification $\left(\mathrm{CH}_{2} \mathrm{Cl}_{2} / \mathrm{CH}_{3} \mathrm{OH} 40 / 1\right)$ a colorless oil (62 mg, 91\% ). $[\alpha]_{D}^{25}-174.7$ (C 0.95, $\left.\mathrm{CH}_{3} \mathrm{OH}\right)$; IR(ATR) 3325,

$3308,3279,3267,2947,2832,1688,1632,1603,1587,1514,1441,1371,1325,1307,1254,1202$, $1165,1144,1103,1065,1018 \mathrm{~cm}^{-1} ;{ }^{1} \mathrm{H}$ NMR $\left(\mathrm{CD}_{3} \mathrm{OD}, 400 \mathrm{MHz}\right): \delta(\mathrm{ppm})=7.65$ and $7.59(2 \mathrm{~d}, 2 \mathrm{H}$, $\left.{ }^{3} J 15.9 \mathrm{~Hz}, 2 \mathrm{x} \mathrm{CH}=\mathrm{CHCO}\right), 7.47$ (d, $\left.4 \mathrm{H}, J_{\text {ortho }} 8.6 \mathrm{~Hz}, \mathrm{Ar}-\mathrm{H}\right), 6.81$ (dd, $4 \mathrm{H}, J_{\text {ortho }} 8.6 \mathrm{~Hz}, J_{\text {meta }} 1.8 \mathrm{~Hz}$ Ar-H), 6.34 and $6.26\left(\mathrm{~d}, 2 \mathrm{H},{ }^{3} \mathrm{~J} 15.9 \mathrm{~Hz}, \mathrm{CH}=\mathrm{CHCO}\right), 5.43\left(\mathrm{~m}, 2 \mathrm{H}, \mathrm{H}_{3}\right.$ and $\left.\mathrm{H}_{5}\right), 3.69$ (s, 3H, OMe), $2.46\left(\mathrm{dd}, 1 \mathrm{H},{ }^{2} J 13.4 \mathrm{~Hz},{ }^{3} J 4.1 \mathrm{~Hz}, \mathrm{H}_{2}, \mathrm{H}_{4}\right.$ or $\left.\mathrm{H}_{6}\right), 2.40\left(\mathrm{dd}, 1 \mathrm{H},{ }^{2} J 13.7 \mathrm{~Hz},{ }^{3} \mathrm{~J} 7.0 \mathrm{~Hz}, \mathrm{H}_{2}, \mathrm{H}_{4}\right.$ or $\left.\mathrm{H}_{6}\right)$, 2.10-1.95 (m, 3H, $\mathrm{H}_{2}, \mathrm{H}_{4}$ or $\left.\mathrm{H}_{6}\right), 1.87\left(\mathrm{dd}, 1 \mathrm{H},{ }^{2} \mathrm{~J} 13.4 \mathrm{~Hz},{ }^{3} \mathrm{~J} 7.6 \mathrm{~Hz} \mathrm{H}_{2}, \mathrm{H}_{4}\right.$ or $\left.\mathrm{H}_{6}\right) .{ }^{13} \mathrm{C} \mathrm{NMR}\left(\mathrm{CD}_{3} \mathrm{OD}\right.$, $100 \mathrm{MHz}) \delta(\mathrm{ppm})=175.7(\mathrm{COOMe}), 168.5(\mathrm{OCO}), 168.0(\mathrm{OCO}), 161.4\left(\mathrm{C}_{\mathrm{q}}\right), 161.3\left(\mathrm{C}_{\mathrm{q}}\right), 146.8$ and $146.7(2 \mathrm{x} \underline{\mathrm{CH}}=\mathrm{CHCO}), 131.23$ and $131.20(2 \times 2 \times C H), 127.2$ and $127.0\left(2 \times \mathrm{C}_{\mathrm{q}}\right), 116.9,116.8(2 \times 2 \mathrm{CH})$, 115.5 and $115.1(2 \times \mathrm{OCOCH}=\underline{\mathrm{CH}}), 75.0\left(\mathrm{C}_{1}\right), 69.5,69.0\left(\mathrm{C}_{3}\right.$ and $\left.\mathrm{C}_{5}\right), 53.0(\mathrm{OMe}), 39.8,39.3$ and 35.9 $\left(3 \mathrm{xCH}_{2}, \mathrm{C}_{2}, \mathrm{C}_{6}\right.$ and $\left.\mathrm{C}_{4}\right)$. HRMS-ESI m/z [M+Na] ${ }^{+}$calcd for $\mathrm{C}_{26} \mathrm{H}_{26} \mathrm{NaO}_{9}$ 505.1469; found 505.1452.

\section{Methyl (3S,5S) 1-hydroxy-3,5 bis[[(2E)-3-(2-hydroxyphenyl)-1-oxo-2-propen-1- yl]oxy]cyclohexanecarboxylate 10e}

The general procedure $\mathrm{E}$ for the deprotection of aromatic acetates was performed with $9 \mathrm{e}(118 \mathrm{mg}$, $0.21 \mathrm{mmol})$ in DMF $(0.9 \mathrm{~mL})$ and hydrazine acetate $(88 \mathrm{mg}, 0.96 \mathrm{mmol})$ providing after purification $\left(\mathrm{CH}_{2} \mathrm{Cl}_{2} / \mathrm{CH}_{3} \mathrm{OH} 40 / 1\right)$ a colorless oil $(89 \mathrm{mg}, 89 \%)$. [ $\left.\alpha\right]_{D}^{25}-104.3$ (C 1.00, $\left.\mathrm{CH}_{3} \mathrm{OH}\right)$; IR(ATR) $3337,3298,3283,3256,2945,2835,1688,1626,1603,1499,1458,1369,1317,1294,1254,1169$, $1146,1113,1067,1018 \mathrm{~cm}^{-1} ;{ }^{1} \mathrm{H}$ NMR $\left(\mathrm{CD}_{3} \mathrm{OD}, 400 \mathrm{MHz}\right): \delta \mathrm{ppm}=$ contaminated with chloroform at $7.90 \mathrm{ppm}, 8.00$ and $7.91\left(2 \mathrm{~d}, 2 \mathrm{H},{ }^{3} J 16.1 \mathrm{~Hz}, \mathrm{CH}=\mathrm{CHCO}\right), 7.48$ (pseudo t, $2 \mathrm{H}, J_{\text {ortho }} 8,2 \mathrm{~Hz} \mathrm{Ar}-\mathrm{H}$ ), 
7.21 (pseudot, 2H, $J_{\text {ortho }} 7.4 \mathrm{~Hz}$ Ar-H), 6.84 (m, 4H, Ar-H), 6.63 and 6.58 (d, 2H, ${ }^{3} J 16.1 \mathrm{~Hz}$, $\mathrm{CH}=\mathrm{CHCO}), 5.45\left(\mathrm{~m}, 2 \mathrm{H}, \mathrm{H}_{3}\right.$ and $\left.\mathrm{H}_{5}\right), 3.72(\mathrm{~s}, 3 \mathrm{H}, \mathrm{OMe}), 2.49\left(\mathrm{dd}, 1 \mathrm{H},{ }^{2} J 13.4 \mathrm{~Hz},{ }^{3} J 3.6 \mathrm{~Hz}, \mathrm{H}_{2}, \mathrm{H}_{4}\right.$ or $\left.\mathrm{H}_{6}\right), 2.43\left(\mathrm{dd}, 1 \mathrm{H},{ }^{2} \mathrm{~J} 13.8 \mathrm{~Hz},{ }^{3} \mathrm{~J} 6.8 \mathrm{~Hz}, \mathrm{H}_{2}, \mathrm{H}_{4}\right.$ or $\mathrm{H}_{6}$ ), 2.15-2.09 (m, $1 \mathrm{H}, \mathrm{H}_{2}, \mathrm{H}_{4}$ or $\mathrm{H}_{6}$ ), 2.00-1.96 $\left(\mathrm{m}, 2 \mathrm{H}, \mathrm{H}_{2}, \mathrm{H}_{4}\right.$ or $\left.\mathrm{H}_{6}\right), 1.86\left(\mathrm{dd}, 1 \mathrm{H},{ }^{2} J 13.3 \mathrm{~Hz},{ }^{3} J 7.9 \mathrm{~Hz}, \mathrm{H}_{2}, \mathrm{H}_{4}\right.$ or $\left.\mathrm{H}_{6}\right) .{ }^{13} \mathrm{C} \mathrm{NMR}\left(\mathrm{CD}_{3} \mathrm{OD}, 100\right.$ $\mathrm{MHz}) \delta(\mathrm{ppm})=$ contaminated with chloroform at $79.4 \mathrm{ppm} ; 175.7$ (COOMe), 168.8(OCO), 168.4(OCO), 158.5 and $158.3\left(2 \mathrm{xC}_{\mathrm{q}}\right), 142.9$ and $142.5(2 \mathrm{xCH}=\mathrm{CHCO}), 132.7,132.6,130.6,130.2$ $\left(4 \mathrm{xCH}_{\mathrm{Ar}}\right), 122.6,122.5\left(2 \mathrm{xC}_{\mathrm{q}}\right), 120.8\left(2 \mathrm{xCH}_{\mathrm{Ar}}\right), 118.6,118.5(2 \mathrm{x} \mathrm{OCOCH}=\mathbf{C H}), 117.0\left(2 \mathrm{xCH}_{\mathrm{Ar}}\right)$, 79.4, $75.0\left(\mathrm{C}_{1}\right), 69.5,69.1\left(\mathrm{C}_{3}\right.$ and $\left.\mathrm{C}_{5}\right), 53.0(\mathrm{OMe}), 39.8,39.4$ and $35.9\left(3 \mathrm{xCH}_{2}, \mathrm{C}_{2}, \mathrm{C}_{6}\right.$ and $\left.\mathrm{C}_{4}\right)$. HRMS-ESI m/z [M+Na] $]^{+}$calcd for $\mathrm{C}_{26} \mathrm{H}_{26} \mathrm{NaO}_{9} 505.1469$; found 505.1448.

\section{(3S,5S) 1-hydroxy-3,5}

bis[[(2E)-3-(3,4-dihydroxyphenyl)-1-oxo-2-propen-1-

\section{yl]oxy]cyclohexanecarboxylic acid 4a}

LiI (143 mg, $1.07 \mathrm{mmol})$ was added to a solution of 9a (146 mg, $0.214 \mathrm{mmol})$ in EtOAc $(1.1 \mathrm{~mL})$ then the mixture was heated under reflux overnight. After cooling down to room temperature, the mixture was diluted with EtOAc $(30 \mathrm{~mL})$, washed with $1 \mathrm{M} \mathrm{HCl}(2 \mathrm{x} 10 \mathrm{~mL})$ and extracted with EtOAc $(3 \times 30 \mathrm{~mL})$. The organic layer was dried over $\mathrm{MgSO}_{4}$, concentrated to give the crude (3S,5S) 1hydroxy-3,5 bis[[(2E)-3-(3,4-diacetoxyphenyl)-1-oxo-2-propen-1-yl]oxy]cyclohexanecarboxylic acid 11a (128 mg, crude yield: 90\%), pure enough for characterization and used directly in the following step. IR(ATR) 3370, 2941, 2833, 1769, 1707, 1638, 1504, 1369, 1240, 1201, 1174, 1143, 1109, 1012 $\mathrm{cm}^{-1} ;{ }^{1} \mathrm{H}$ NMR $\left(\mathrm{CD}_{3} \mathrm{OD}, 298 \mathrm{~K}, 400 \mathrm{MHz}\right): \delta \mathrm{ppm}=7.67$ and $7.62(2 \mathrm{~d}, 2 \mathrm{H}, J 16.0 \mathrm{~Hz}, \mathrm{C} \underline{\mathrm{H}}=\mathrm{CHCO})$, $7.50\left(\mathrm{~m}, 2 \mathrm{H}, \mathrm{H}_{6}{ }^{\prime}\right), 7.49\left(\mathrm{~m}, 2 \mathrm{H}, \mathrm{H}_{2}{ }^{\prime}\right), 7.24\left(\mathrm{~d}, 2 \mathrm{H}, J_{\text {ortho }} 8.2 \mathrm{~Hz}, \mathrm{H}_{5}{ }^{\prime}\right), 6.52$ and $6.46(2 \mathrm{~d}, 2 \mathrm{H}, J 16.0 \mathrm{~Hz}$, $\mathrm{CH}=\mathrm{CHCO}), 5.46\left(\mathrm{~m}, 2 \mathrm{H}, \mathrm{H}_{3}\right.$ and $\left.\mathrm{H}_{5}\right), 2.44-2.19(\mathrm{~m}, 14 \mathrm{H})$ and $2.06-1.90\left(\mathrm{~m}, 4 \mathrm{H}, 3 \mathrm{xCH}_{2}\right.$ and $\left.4 \mathrm{xOCOCH}_{3}\right) ;{ }^{13} \mathrm{C} \mathrm{NMR}\left(\mathrm{CD}_{3} \mathrm{OD}, 298 \mathrm{~K}, 100 \mathrm{MHz}\right) \delta(\mathrm{ppm})=177.3(\mathrm{COOH}), 169.8$ and $169.7(4 \mathrm{x}$ $\left.\mathrm{OCOCH}_{3}\right), 167.5$ and $167.2(2 \mathrm{xOCO}), 145.2$ and $145.1\left(2 \mathrm{xC}_{\mathrm{q}}\right), 144.5$ and $144.4(2 \mathrm{x} \underline{\mathrm{CH}}=\mathrm{CHCO})$, $144.0\left(2 \mathrm{xC}_{\mathrm{q}}\right), 134.60$ and $134.56\left(2 \mathrm{xC}_{\mathrm{q}}\right), 127.7$ and $127.6\left(2 \mathrm{xC}_{6}\right), 125.13$ and $125.11\left(2 \mathrm{xC}_{5}\right), 124.14$ and $124.09\left(\mathrm{C}_{2}\right), 120.45$ and $120.13(2 \mathrm{x} \mathrm{CH}=\underline{\mathrm{CHCO}}), 75.0\left(\mathrm{C}_{1}\right), 70.2,69.2\left(\mathrm{C}_{3}\right.$ and $\left.\mathrm{C}_{5}\right), 39.9,38.7$, $35.9\left(\mathrm{C}_{2}, \mathrm{C}_{4}, \mathrm{C}_{6}\right), 20.5\left(4 \mathrm{XOCOC} \mathrm{H}_{3}\right)$. HRMS-ESI m/z $[\mathrm{M}+\mathrm{H}]^{+}$calcd for $\mathrm{C}_{33} \mathrm{H}_{33} \mathrm{O}_{15} 669.1814$; found 669.1801 .

To a solution of (3S,5S) 1-hydroxy-3,5 bis[[(2E)-3-(3,4-diacetoxyphenyl)-1-oxo-2-propen-1yl]oxy]cyclohexanecarboxylic acid 11a $(208 \mathrm{mg})$ in acetone $(4.08 \mathrm{~mL})$ was added $3 \mathrm{M} \mathrm{HCl}$ aqueous solution $(2.0 \mathrm{~mL})$ and the mixture was refluxed for $5 \mathrm{~h}$. After completion, the reaction mixture was cooled down to room temperature, diluted with EtOAc $(30 \mathrm{~mL})$ and washed with brine $(20 \mathrm{~mL})$. The organic layer was concentrated to give the crude quantitatively as a pale solid $4 \mathbf{a}$ (190 $\mathrm{mg}$ ).

$[\alpha]_{D}^{25}-42.7\left(C\right.$ 0.82, $\left.\mathrm{CH}_{3} \mathrm{OH}\right) ;{ }^{1} \mathrm{H}$ NMR $\left(\mathrm{CD}_{3} \mathrm{OD}, 400 \mathrm{MHz}, 295 \mathrm{~K}\right): \operatorname{IR}(\mathrm{ATR}) 3345,3210,1687$, $1603,1523,1445,1362,1279,1182,1138,1119 \mathrm{~cm}^{-1} ; \delta(\mathrm{ppm})=7.65$ and $7.60(2 \mathrm{~d}, 2 \mathrm{H}, J 16.0 \mathrm{~Hz}$, $\mathrm{C} \underline{\mathrm{H}}=\mathrm{CHCO}), 7.45(\mathrm{~m}, 4 \mathrm{H}, \mathrm{ArH}), 6.81(\mathrm{~m}, 4 \mathrm{H}, \mathrm{ArH}), 6.34$ and $6.27(2 \mathrm{~d}, 2 \mathrm{H}, J 16.0 \mathrm{~Hz}, \mathrm{CH}=\mathrm{CHCO})$, $5.45\left(\mathrm{~m}, 2 \mathrm{H}, \mathrm{H}_{3}\right.$ and $\left.\mathrm{H}_{5}\right), 2.41\left(\mathrm{dd}, 1 \mathrm{H},{ }^{2} J 14.0 \mathrm{~Hz},{ }^{3} \mathrm{~J} 3.8 \mathrm{~Hz}, \mathrm{H}_{2}, \mathrm{H}_{4}\right.$ or $\left.\mathrm{H}_{6}\right), 2.32\left(\mathrm{dd}, 1 \mathrm{H},{ }^{2} J 13.3 \mathrm{~Hz}\right.$, ${ }^{3} J 8.2 \mathrm{~Hz}, \mathrm{H}_{2}, \mathrm{H}_{4}$ or $\left.\mathrm{H}_{6}\right), 2.06\left(\mathrm{~m}, 3 \mathrm{H}, \mathrm{H}_{2}, \mathrm{H}_{4}\right.$ or $\left.\mathrm{H}_{6}\right), 1.93\left(\mathrm{dd}, 1 \mathrm{H},{ }^{2} J 13.8 \mathrm{~Hz},{ }^{3} J 6.5 \mathrm{~Hz}, \mathrm{H}_{2}, \mathrm{H}_{4}\right.$ or $\mathrm{H}_{6}$ ); ${ }^{13} \mathrm{C} \mathrm{NMR}\left(\mathrm{CD}_{3} \mathrm{OD}, 100 \mathrm{MHz}, 297 \mathrm{~K}\right) \delta(\mathrm{ppm})=177.4(\mathrm{COOH}), 171.0\left(2 \mathrm{xC}_{\mathrm{q}}\right), 168.5$ and 168.3 (2xOCO), 161.12 and $161.07(2 x C q), 146.7$ and $146.7(2 \mathrm{x} \underline{\mathrm{CH}}=\mathrm{CHCO}), 131.2,131.1\left(2 \mathrm{xCH}_{\mathrm{Ar}}\right), 127.1$ 
$\left(2 \mathrm{xC}_{\mathrm{q}}\right), 116.8$ (4xCHAr), 115.5, $115.1(2 \mathrm{x} \mathrm{CH}=\mathrm{CHCO}), 75.1\left(\mathrm{C}_{1}\right), 69.9,68.7\left(\mathrm{C}_{3}\right.$ and $\left.\mathrm{C}_{5}\right), 39.9,38.6$, $35.9\left(\mathrm{C}_{2}, \mathrm{C}_{4}, \mathrm{C}_{6}\right)$.

(3S,5S) 1-hydroxy-3,5 bis[[(2E)-3-(4-hydroxy-3-methoxyphenyl)-1-oxo-2-propen-1yl]oxy]cyclohexanecarboxylic acid $4 c$

LiI (152 mg, $1.135 \mathrm{mmol})$ was added to a solution of 9c (142 $\mathrm{mg}, 0.2274 \mathrm{mmol})$ in EtOAc $(5.0 \mathrm{~mL})$ then the mixture was heated under reflux $24 \mathrm{~h}$. After cooling down to room temperature, the mixture was diluted with EtOAc $(25 \mathrm{~mL})$, washed with $3 \mathrm{M} \mathrm{HCl}(5 \mathrm{~mL})$ and extracted with EtOAc $(3 \times 30 \mathrm{~mL})$. The organic layer was dried over $\mathrm{MgSO}_{4}$, concentrated to give the crude (3S,5S) 1-hydroxy-3,5 bis[[(2E)-3-(4-acetoxy-3-methoxyphenyl)-1-oxo-2-propen-1-yl]oxy]cyclohexanecarboxylic acid 11c (quantitative crude yield), pure enough for characterization and used directly in the following step.

$[\alpha]_{D}^{25}-97.5\left(C\right.$ 0.96, $\left.\mathrm{CH}_{3} \mathrm{OH}\right)$; IR(ATR) 3466, 2941, 2833, 1763, 1705, 1599, 1508, 1464, 1454, 1418, $1369,1256,1215,1177,1152,1063,1020 \mathrm{~cm}^{-1} ;{ }^{1} \mathrm{H} \mathrm{NMR}\left(\mathrm{CDCl}_{3}, 400 \mathrm{MHz}\right): \delta(\mathrm{ppm})=7.66$ and $7.61\left(2 \mathrm{~d}, 2 \mathrm{H},{ }^{3} \mathrm{~J} 15.9 \mathrm{~Hz}, \mathrm{CH}=\mathrm{CHCO}\right), 7.13-7.03(2 \mathrm{~m}, 6 \mathrm{H}, \mathrm{Ar}-\mathrm{H}), 6.41$ and $6.34\left(2 \mathrm{~d}, 2 \mathrm{H},{ }^{3} J 15.9 \mathrm{~Hz}\right.$, $\mathrm{CH}=\mathrm{CHCO}), 5.50\left(\mathrm{~m}, 2 \mathrm{H}, \mathrm{H}_{3}\right.$ and $\left.\mathrm{H}_{5}\right), 3.87$ and $3.85(2 \mathrm{~s}, 2 \times 3 \mathrm{H}, 2 \mathrm{xOMe}), 2.33-1.83\left(\mathrm{~m}, 12 \mathrm{H}, \mathrm{H}_{2}, \mathrm{H}_{4}\right.$, $\mathrm{H}_{6}$ and $\left.2 \mathrm{xOCOCH}_{3}\right) ;{ }^{13} \mathrm{C} \mathrm{NMR}\left(\mathrm{CDCl}_{3}, 100 \mathrm{MHz}\right) \delta(\mathrm{ppm})=176.7(\mathrm{COOH}), 169.04$ and 169.02 $\left(2 \mathrm{xOCOCH}_{3}\right), 166.1$ and $166.0(2 \mathrm{xCOO}), 151.53$ and $151.50\left(2 \mathrm{xC}_{\mathrm{q}}\right), 145.3,144.8(2 \mathrm{x} \underline{\mathrm{CH}}=\mathrm{CHCO})$, 141.7, $141.6\left(2 \mathrm{xC}_{\mathrm{q}}\right), 133.3,133.2\left(2 \mathrm{xC}_{\mathrm{q}}\right), 123.4\left(2 \mathrm{xCH}_{\mathrm{Ar}}\right), 121.8,121.4(2 \mathrm{x} \mathrm{CH}=\mathrm{CHCO}), 118.1,117.9$ $\left(2 \mathrm{xCH}_{\mathrm{Ar}}\right), 111.4,111.3\left(2 \mathrm{xCH}_{\mathrm{Ar}}\right), 75.2\left(\mathrm{C}_{1}\right), 69.4,66.5\left(\mathrm{C}_{3}\right.$ and $\left.\mathrm{C}_{5}\right), 56.1,56.0(2 \mathrm{xOMe}), 39.3,36.5$, $34.9\left(\mathrm{C}_{2}, \mathrm{C}_{4}, \mathrm{C}_{6}\right), 20.8\left(2 \mathrm{xOCO} \underline{C H}_{3}\right) ; \mathrm{HRMS}-\mathrm{ESI} \mathrm{m} / \mathrm{z}[\mathrm{M}+\mathrm{Na}]^{+}$calcd for $\mathrm{C}_{31} \mathrm{H}_{32} \mathrm{NaO}_{13} 635.1735$; found 635.1706 .

To a solution of (3S,5S) 1-hydroxy-3,5 bis[[(2E)-3-(4-acetoxy-3-methoxyphenyl)-1-oxo-2-propen-1yl]oxy]cyclohexanecarboxylic acid $11 \mathrm{c}(176 \mathrm{mg})$ in acetone $(3.0 \mathrm{~mL})$ was added $3 \mathrm{M} \mathrm{HCl}$ aqueous solution $(1.5 \mathrm{~mL})$ and the mixture was refluxed for $4 \mathrm{~h}$. After completion, the reaction mixture was cooled down to room temperature, diluted with EtOAc $(30 \mathrm{~mL})$ and washed with brine $(20 \mathrm{~mL})$. The organic layer was concentrated then dissolved in $\mathrm{MeOH} / \mathrm{H}_{2} \mathrm{O}(30 / 40 \mathrm{~mL})$ and washed with pentane ( $2 \times 15 \mathrm{~mL})$. After concentration, the crude was obtained as a pale solid $4 \mathbf{c}(150 \mathrm{mg})$.

$[\alpha]_{D}^{25}-94.4\left(C 0.67, \mathrm{CH}_{3} \mathrm{OH}\right)$; IR(ATR) 3356, 3277, 1684, 1632, 1605, 1514, 1443, 1327, 1261, 1204,

1171, 1138, $1007 \mathrm{~cm}^{-1} ;{ }^{1} \mathrm{H}$ NMR $\left(\mathrm{CD}_{3} \mathrm{OD}, 300 \mathrm{MHz}, 297 \mathrm{~K}\right): \delta(\mathrm{ppm}) 7.65$ and $7.60(2 \mathrm{~d}, 2 \mathrm{H}, J 15.9$ $\mathrm{Hz}, \mathrm{CH}=\mathrm{CHCO}), 7.17(\mathrm{~m}, 2 \mathrm{H}, \mathrm{ArH}), 7.07(\mathrm{~m}, 2 \mathrm{H}, \mathrm{ArH}), 6.83\left(\mathrm{~d}, 2 \mathrm{H}, J_{\text {ortho }} 8.1 \mathrm{~Hz}, \mathrm{ArH}\right), 6.39$ and $6.32(2 \mathrm{~d}, 2 \mathrm{H}, J 15.9 \mathrm{~Hz}, \mathrm{CH}=\mathrm{CHCO}), 5.47\left(\mathrm{~m}, 2 \mathrm{H}, \mathrm{H}_{3}\right.$ and $\left.\mathrm{H}_{5}\right), 3.89(\mathrm{~s}, 6 \mathrm{H}, 2 \mathrm{xOMe}), 2.42\left(\mathrm{dd}, 1 \mathrm{H},{ }^{2} J\right.$ $13.9 \mathrm{~Hz},{ }^{3} \mathrm{~J} 4.0 \mathrm{~Hz}, \mathrm{H}_{2}, \mathrm{H}_{4}$ or $\mathrm{H}_{6}$ ), 2.34 (dd, $1 \mathrm{H},{ }^{2} J 13.4 \mathrm{~Hz},{ }^{3} \mathrm{~J} 8.2 \mathrm{~Hz}, \mathrm{H}_{2}, \mathrm{H}_{4}$ or $\left.\mathrm{H}_{6}\right), 2.00\left(\mathrm{~m}, 4 \mathrm{H}, \mathrm{H}_{2}\right.$, $\mathrm{H}_{4}$ or $\left.\mathrm{H}_{6}\right) ;{ }^{13} \mathrm{C} \mathrm{NMR}\left(\mathrm{CD}_{3} \mathrm{OD}, 75 \mathrm{MHz}, 297 \mathrm{~K}\right) \delta(\mathrm{ppm})=177.4(\mathrm{COOH}), 170.9\left(2 \mathrm{xC}_{\mathrm{q}}\right), 168.5$ and $168.2(2 \mathrm{xOCO}), 150.44,150.38\left(2 \mathrm{xC}_{\mathrm{q}}\right), 146.9$ and $146.8(2 \mathrm{x} \underline{\mathrm{CH}}=\mathrm{CHCO}), 127.74$ and $127.68\left(2 \mathrm{xC}_{\mathrm{q}}\right)$, 124.1, 124.0, 123.9, 116.4, 115.8, $115.5\left(6 \mathrm{x} \mathrm{CH}_{\mathrm{Ar}}\right), 111.7$ and $111.6(2 \mathrm{x} \mathrm{CH}=\underline{\mathrm{CHCO}}), 75.1\left(\mathrm{C}_{1}\right), 69.9$, $68.7\left(\mathrm{C}_{3}\right.$ and $\left.\mathrm{C}_{5}\right), 56.43$ and $56.41(2 \mathrm{xOMe}), 40.0,38.7,36.0\left(\mathrm{C}_{2}, \mathrm{C}_{4}, \mathrm{C}_{6}\right)$. 


\section{yl]oxy]cyclohexanecarboxylic acid $4 d$}

LiI (125 mg, $0.934 \mathrm{mmol})$ was added to a solution of 9d (105 mg, $0.186 \mathrm{mmol})$ in EtOAc $(4.0 \mathrm{~mL})$ then the mixture was heated under reflux $24 \mathrm{~h}$. After cooling down to room temperature, the mixture was diluted with EtOAc $(25 \mathrm{~mL})$, washed with $3 \mathrm{M} \mathrm{HCl}(5 \mathrm{~mL})$ and extracted with EtOAc $(3 \times 30 \mathrm{~mL})$. The organic layer was dried over $\mathrm{MgSO}_{4}$, concentrated to give the crude (3S,5S) 1-hydroxy-3,5 bis[[(2E)-3-(4-acetoxyphenyl)-1-oxo-2-propen-1-yl]oxy]cyclohexanecarboxylic acid 11d (quantitative crude yield), pure enough for characterization and used directly in the following step. $[\alpha]_{D}^{25}-111.9$ (C

1.03, $\mathrm{CH}_{3} \mathrm{OH}$ ); IR(ATR) 3447, 3059, 2961, 1765, 1707, 1601, 1585, 1506, 1418, 1369, 1312, 1265, $1202,1163,1136,1057,1009 \mathrm{~cm}^{-1} ;{ }^{1} \mathrm{H}$ NMR $\left(\mathrm{CDCl}_{3}, 400 \mathrm{MHz}\right): \delta \mathrm{ppm}=7.68$ and $7.62\left(2 \mathrm{~d}, 2 \mathrm{H},{ }^{3} \mathrm{~J}\right.$ $15.9 \mathrm{~Hz}, \mathrm{CH}=\mathrm{CHCO}), 7.52\left(\mathrm{dd}, 4 \mathrm{H}, J_{\text {ortho }} 8,6 \mathrm{~Hz}, J_{\text {ortho }} 6.8 \mathrm{~Hz}, \mathrm{Ar}-\mathrm{H}\right), 7.11$ (dd, $4 \mathrm{H}, J_{\text {ortho }} 8,6 \mathrm{~Hz}, J_{\text {meta }}$ $2.0 \mathrm{~Hz}, \mathrm{Ar}-\mathrm{H}), 6.41$ and $6.34\left(2 \mathrm{~d}, 2 \mathrm{H},{ }^{3} \mathrm{~J} 15.9 \mathrm{~Hz}, \mathrm{CH}=\mathrm{CHCO}\right), 5.49\left(\mathrm{~m}, 2 \mathrm{H}, \mathrm{H}_{3}\right.$ and $\left.\mathrm{H}_{5}\right), 2.36-2.02$

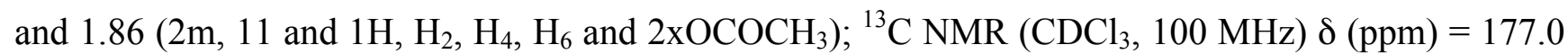
$(\mathrm{COOH}), 169.41$ and $169.39\left(2 \mathrm{xOCOCH}_{3}\right), 166.2,166.1(2 \mathrm{xCOO}), 152.4,152.3\left(2 \mathrm{xC}_{\mathrm{q}}\right), 144.9,144.4$ $(2 \mathrm{xCH}=\mathrm{CHCO}), 132.04,132.0\left(2 \mathrm{xC}_{\mathrm{q}}\right), 129.6,129.5\left(4 \mathrm{xCH}_{\mathrm{Ar}}\right), 122.3\left(4 \mathrm{xCH}_{\mathrm{Ar}}\right), 118.0,117.9(2 \mathrm{x}$ $\mathrm{CH}=\underline{\mathrm{CHCO}})$, $75.2\left(\mathrm{C}_{1}\right), 69.4,66.6\left(\mathrm{C}_{3}\right.$ and $\left.\mathrm{C}_{5}\right), 39.3,36.5,34.9\left(\mathrm{C}_{2}, \mathrm{C}_{4}\right.$ and $\left.\mathrm{C}_{6}\right), 21.3\left(2 \mathrm{x} \mathrm{OCOCH}_{3}\right)$; HRMS-ESI m/z [M+Na] $]^{+}$calcd for $\mathrm{C}_{29} \mathrm{H}_{28} \mathrm{NaO}_{11}$ 575.1524; found 575.1503.

To a solution of (3S,5S) 1-hydroxy-3,5 bis[[(2E)-3-(4-acetoxyphenyl)-1-oxo-2-propen-1yl]oxy]cyclohexanecarboxylic acid $11 d(196 \mathrm{mg})$ in acetone $(4.0 \mathrm{~mL})$ was added $3 \mathrm{M} \mathrm{HCl}$ aqueous solution $(2.0 \mathrm{~mL})$ and the mixture was refluxed for $5 \mathrm{~h}$. After completion, the reaction mixture was cooled down to room temperature, diluted with EtOAc $(30 \mathrm{~mL})$ and washed with brine $(20 \mathrm{~mL})$. The organic layer was concentrated, dissolved in $\mathrm{MeOH} / \mathrm{H}_{2} \mathrm{O}(20 / 50 \mathrm{~mL})$ and washed with pentane $(2 \mathrm{x}$ $15 \mathrm{~mL})$. After concentration, the crude quantitatively as a pale solid $\mathbf{4 d}(165 \mathrm{mg})$.

$[\alpha]_{D}^{25}-72.6\left(C\right.$ 0.71, $\left.\mathrm{CH}_{3} \mathrm{OH}\right)$; IR (ATR) 3406, 2976, 2947, 1692, 1593, 1514, 1456, 1429, 1375, 1269 , $1209,1177,1161,1138,1032 \mathrm{~cm}^{-1} ;{ }^{1} \mathrm{H}$ NMR $\left(\mathrm{CD}_{3} \mathrm{OD}, 400 \mathrm{MHz}, 295 \mathrm{~K}\right) \delta(\mathrm{ppm})=7.59$ and $7.54(2 \mathrm{~d}$, $2 \mathrm{H}, J 16.0 \mathrm{~Hz}, \mathrm{CH}=\mathrm{CHCO}), 7.08(\mathrm{~m}, 4 \mathrm{H}, \mathrm{ArH}), 6.97-6.92(\mathrm{~m}, 4 \mathrm{H}, \mathrm{ArH}), 6.81(\mathrm{~m}, 2 \mathrm{H}, \mathrm{ArH}), 6.29$ and $6.23(2 \mathrm{~d}, 2 \mathrm{H}, J 16.0 \mathrm{~Hz}, \mathrm{CH}=\mathrm{CHCO}), 5.44\left(\mathrm{~m}, 2 \mathrm{H}, \mathrm{H}_{3}\right.$ and $\left.\mathrm{H}_{5}\right), 2.40\left(\mathrm{dd}, 1 \mathrm{H},{ }^{2} J 13.9 \mathrm{~Hz},{ }^{3} J 4.3 \mathrm{~Hz}\right.$, $\mathrm{H}_{2}, \mathrm{H}_{4}$ or $\left.\mathrm{H}_{6}\right), 2.32\left(\mathrm{dd}, 1 \mathrm{H},{ }^{2} J 13.4 \mathrm{~Hz},{ }^{3} J 8.2 \mathrm{~Hz}, \mathrm{H}_{2}, \mathrm{H}_{4}\right.$ or $\left.\mathrm{H}_{6}\right), 1.96\left(\mathrm{~m}, 4 \mathrm{H}, \mathrm{H}_{2}, \mathrm{H}_{4}\right.$ or $\left.\mathrm{H}_{6}\right) ;{ }^{13} \mathrm{C} \mathrm{NMR}$ $\left(\mathrm{CD}_{3} \mathrm{OD}, 100 \mathrm{MHz}, 297 \mathrm{~K}\right) \delta(\mathrm{ppm})=177.4(\mathrm{COOH}), 171.0\left(2 \mathrm{xC}_{\mathrm{q}}\right), 168.6$ and $168.5(2 \mathrm{xOCO}), 149.4$, $149.3\left(2 \mathrm{xC}_{\mathrm{q}}\right), 147.1$ and $146.9(2 \mathrm{x} \underline{\mathrm{CH}}=\mathrm{CHCO}), 127.74$ and $127.68\left(2 \mathrm{xC}_{\mathrm{q}}\right), 123.1,123.0,116.5,116.4$ (x2), 115.4, .115.2, 115.11, 115.09, $115.0\left(8 \mathrm{x} \mathrm{CH}_{\mathrm{Ar}}+2 \mathrm{x} \mathrm{CH}=\underline{\mathrm{C} H C O}\right), 75.0\left(\mathrm{C}_{1}\right), 69.9,68.7\left(\mathrm{C}_{3}\right.$ and $\left.\mathrm{C}_{5}\right), 39.9,38.6,35.9\left(\mathrm{C}_{2}, \mathrm{C}_{4}, \mathrm{C}_{6}\right)$.

\section{Synthesis of $(3 R, 3 a R, 6 S, 6 a R)$-hexahydrofuro[3,2-b]furan-3-yl (2E)-3-(3,4-dihydroxyphenyl)prop- 2-enoate (12) ${ }^{11}$}

\footnotetext{
${ }^{11}$ To the best of our knowledge, the only reported syntheses of this family of compounds (dicinnamate and di (pcoumarate) isosorbide) used DCC/DMAP methodology. W. Huang, Y. B. Cao, X. G. Zhang, F. S. Li and H. Yang, Chin. Chem. Lett., 2009, 20, 873-876 ; A. Y. Bobrovsky, N. I. Boiko and V. P. Shibaev, Mol. Cryst. Liq. Cryst., 2001, 363, $35-$ 50 .
} 


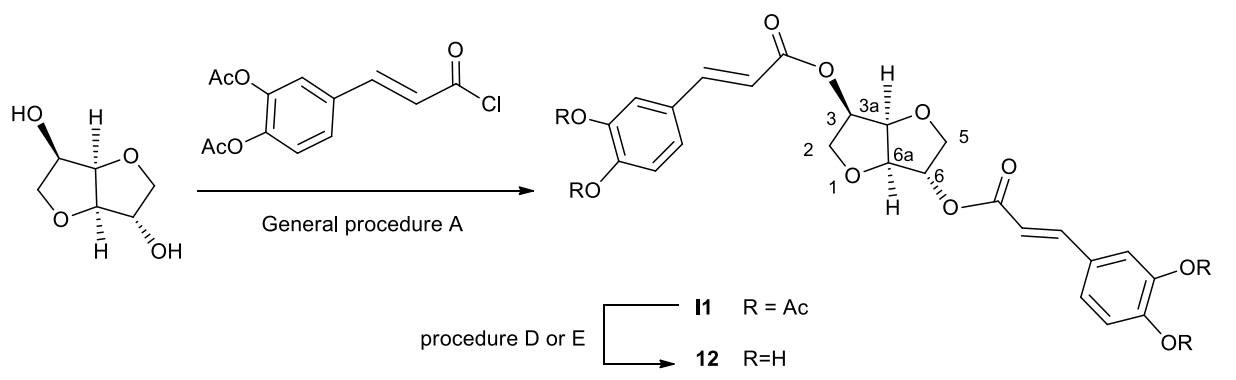

Isosorbide dicaffeate was also synthesized in our lab using the general procedure A with isosorbide (300 $\mathrm{mg}, 2.05 \mathrm{mmol})$ in anhydrous pyridine $(2.1 \mathrm{~mL})$ and DMF $(3.2 \mathrm{~mL})$ and diacetylacaffeoylchloride (1.51 g, $5.36 \mathrm{mmol}, 2.6$ eq.). (3R, 3aR, 6S, 6aR)-hexahydrofuro[3,2-b]furan3,6-diyl (2E,2'E)bis-[3,4-bis(acetyloxy)phenyl]prop-2-enoate I1 was obtained as a white solid (414mg, $32 \%$ ) was obtained after purification by flash chromatography (pentane/EtOAc 6/4).

$[\alpha]_{D}^{25}-59$ (c 1.00, $\left.\mathrm{CH}_{2} \mathrm{Cl}_{2}\right)$; m.p.69-70 ${ }^{\circ} \mathrm{C}\left(\mathrm{CH}_{2} \mathrm{Cl}_{2}\right)$; IR (ATR) $\mathrm{cm}^{-1} 1767,1707,1638,1502,1242$, 1204, 1168, 1110; ${ }^{1} \mathrm{H}$ NMR $\left(400 \mathrm{MHz}, \mathrm{CDCl}_{3}\right) \delta(\mathrm{ppm})=7.67$ and $7.63\left(2 \mathrm{~d}, 2 \mathrm{H},{ }^{3} J 16.0 \mathrm{~Hz}\right.$, $\mathrm{CH}=\mathrm{CHCO}), 7.40-7.35(\mathrm{~m}, 4 \mathrm{H}, \mathrm{ArH}), 7.26-7.21(\mathrm{~m}, 2 \mathrm{H}, \mathrm{ArH}), 6.44$ and $6.36\left(2 \mathrm{~d}, 2 \mathrm{H},{ }^{3} J 16.0 \mathrm{~Hz}\right.$, $\mathrm{CH}=\mathrm{CHCO}$ ), 5.35 (bs, $\left.1 \mathrm{H}, \mathrm{H}_{6}\right), 5.29\left(\mathrm{q}, 1 \mathrm{H}, J_{\mathrm{H}_{3} / \mathrm{H}_{2}} \approx J_{\mathrm{H}_{3 \mathrm{a}} / \mathrm{H}_{3}} \approx 5.5 \mathrm{~Hz}, \mathrm{H}_{3}\right), 4.94\left(\mathrm{dd}, 1 \mathrm{H}, J_{\mathrm{H}_{3 \mathrm{a}} / \mathrm{H}_{6 \mathrm{a}}} \approx\right.$ $\left.J_{\mathrm{H}_{3 \mathrm{a}} / \mathrm{H}_{3}} 4.6 \mathrm{~Hz}, \mathrm{H}_{3 \mathrm{a}}\right), 4.59\left(\mathrm{~d}, 1 \mathrm{H}, J_{\mathrm{H}_{3 \mathrm{a}} / \mathrm{H}_{6 \mathrm{a}}} 4.6 \mathrm{~Hz}, \mathrm{H}_{6 \mathrm{a}}\right), 4.06\left(\mathrm{~m}, 2 \mathrm{H}, 2 \mathrm{HH}_{5}\right), 4.03\left(\mathrm{dd}, 1 \mathrm{H},{ }^{2} J 9.9\right.$ and ${ }^{3} J$ $\left.6.0 \mathrm{~Hz}, \mathrm{H}_{2}\right), 3.90\left(\mathrm{dd}, 1 \mathrm{H},{ }^{2} J 9.8\right.$ and $\left.{ }^{3} J 5.4 \mathrm{~Hz}, \mathrm{H}_{2}\right), 2.292,2.294,2.298,2.302\left(4 \mathrm{~s}, 12 \mathrm{H}, 3 \mathrm{xCH}_{3}\right) ;{ }^{13} \mathrm{C}$ NMR $\left(100 \mathrm{MHz}, \mathrm{CDCl}_{3}\right) \delta(\mathrm{ppm})=168.15$ and $168.06\left(4 \underline{\mathrm{COCH}}_{\underline{3}}\right), 165.9$ and $165.6(2 \mathrm{COO}), 144.03$ and $143.98(2 \mathrm{CH}=\mathrm{CH}), 143.82$ and $143.77\left(2 \mathrm{C}_{\mathrm{q} \mathrm{Ar}}\right), 142.5\left(2 \mathrm{C}_{\mathrm{q} \mathrm{Ar}}\right), 133.1$ and $133.0\left(2 \mathrm{C}_{\mathrm{q} \mathrm{Ar}}\right), 126.6$, 124.1, and $123.0\left(6 \mathrm{CH}_{\mathrm{Ar}}\right), 118.5$ and $118.4(2 \mathrm{CH}=\mathrm{CH}), 86.1\left(\mathrm{C}_{6 \mathrm{a}}\right), 81.1\left(\mathrm{C}_{3 \mathrm{a}}\right), 78.3\left(\mathrm{C}_{6}\right), 74.3\left(\mathrm{C}_{3}\right)$, $73.6\left(\mathrm{C}_{5}\right), 70.5\left(\mathrm{C}_{2}\right), 20.8$ and $20.7\left(4 \mathrm{COCH}_{3}\right)$; HRMS (ESI) calcd for $\mathrm{C}_{32} \mathrm{H}_{30} \mathrm{NaO}_{14}[\mathrm{M}+\mathrm{Na}]^{+}$ 661.1528 , found 661.1537 .

I1 was submitted to general procedure D for deprotection : I1 (144 mg, $0.225 \mathrm{mmol})$ in ethanol (1.2 $\mathrm{mL})$ wih $\mathrm{NaOAc}(377 \mathrm{mg}, 20 \mathrm{eq})$ in water $(600 \mu \mathrm{L}) .12$ was obtained as a white solid $(74 \mathrm{mg}, 72 \%)$ after purification by flash chromatography $\left(\mathrm{CH}_{2} \mathrm{Cl}_{2} / \mathrm{MeOH} 97 / 3-95 / 5\right)$.

$[\alpha]_{D}^{25}-191\left(\mathrm{c} 0.99, \mathrm{CH}_{2} \mathrm{Cl}_{2}\right.$ ); m.p. $192-193^{\circ} \mathrm{C}(\mathrm{MeOH})$; IR (ATR) $\mathrm{cm}^{-1} 3670,3660,2986,2972,2901$, 2370, 2322, 1688, 1601, 1379, 1049; ${ }^{1} \mathrm{H}$ NMR (300 MHz, $\left.\mathrm{CD}_{3} \mathrm{OD}\right) \delta(\mathrm{ppm})=7.58$ and $7.55\left(2 \mathrm{~d},{ }^{3} J\right.$ $15.9 \mathrm{~Hz}, \mathrm{CH}=\mathrm{CHCO}), 7.05(\mathrm{dd}, 2 \mathrm{H}, J 6.1 \mathrm{~Hz}, J 2.0 \mathrm{~Hz}, \mathrm{ArH}) ; 6.94(\mathrm{~m}, 2 \mathrm{H}, \mathrm{ArH}), 6.77$ (dd, $2 \mathrm{H}, J 8.1$ $\mathrm{Hz}, J 3.1 \mathrm{~Hz}, \mathrm{ArH}), 6.29$ and $6.24\left(2 \mathrm{~d}, 2 \mathrm{H},{ }^{3} J 15.9 \mathrm{~Hz}, \mathrm{CH}=\mathrm{CHCO}\right), 5.26\left(\mathrm{~m}, 2 \mathrm{H}, \mathrm{H}_{3}, \mathrm{H}_{6}\right), 4.92$ (dd, $\left.1 \mathrm{H}, J_{\mathrm{H}_{3 \mathrm{a}} / \mathrm{H}_{6 \mathrm{a}}} \approx J_{\mathrm{H}_{3 \mathrm{a}} / \mathrm{H}_{3}} 5.0 \mathrm{~Hz}, \mathrm{H}_{3 \mathrm{a}}\right), 4.53\left(\mathrm{~d}, 1 \mathrm{H}, J_{\mathrm{H}_{3 \mathrm{a}} / \mathrm{H}_{6 \mathrm{a}}} 5.0 \mathrm{~Hz}, \mathrm{H} 6 \mathrm{a}\right), 3.95\left(\mathrm{~m}, 4 \mathrm{H}, \mathrm{H}_{2}, \mathrm{H}_{5}\right) ;{ }^{13} \mathrm{C} \mathrm{NMR}$ $\left(75 \mathrm{MHz}, \mathrm{CD}_{3} \mathrm{OD}\right) \delta(\mathrm{ppm})=168.4,168.1,149.71,149.66,147.7,147.5,146.8,127.6,127.5,123.2$, 123.1, 116.5, 115.2, 114.4, 87.3, 82.6, 79.2, 75.5, 74.2, 71.8; HRMS (ESI) calcd for $\mathrm{C}_{24} \mathrm{H}_{22} \mathrm{NaO}_{10}$ $[\mathrm{M}+\mathrm{Na}]^{+} 493.1105$, found 493.1087 . 
Methyl (3R, 5R)-1,3,4,5-tetrahydroxycyclohexanecarboxylate Q1

${ }^{1} \mathrm{H}$ NMR (300MHz, $\left.\mathrm{CD}_{3} \mathrm{OD}, 300 \mathrm{~K}\right)$

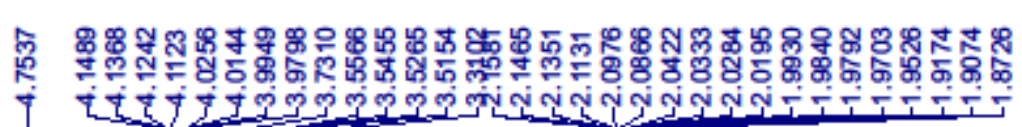

OOH
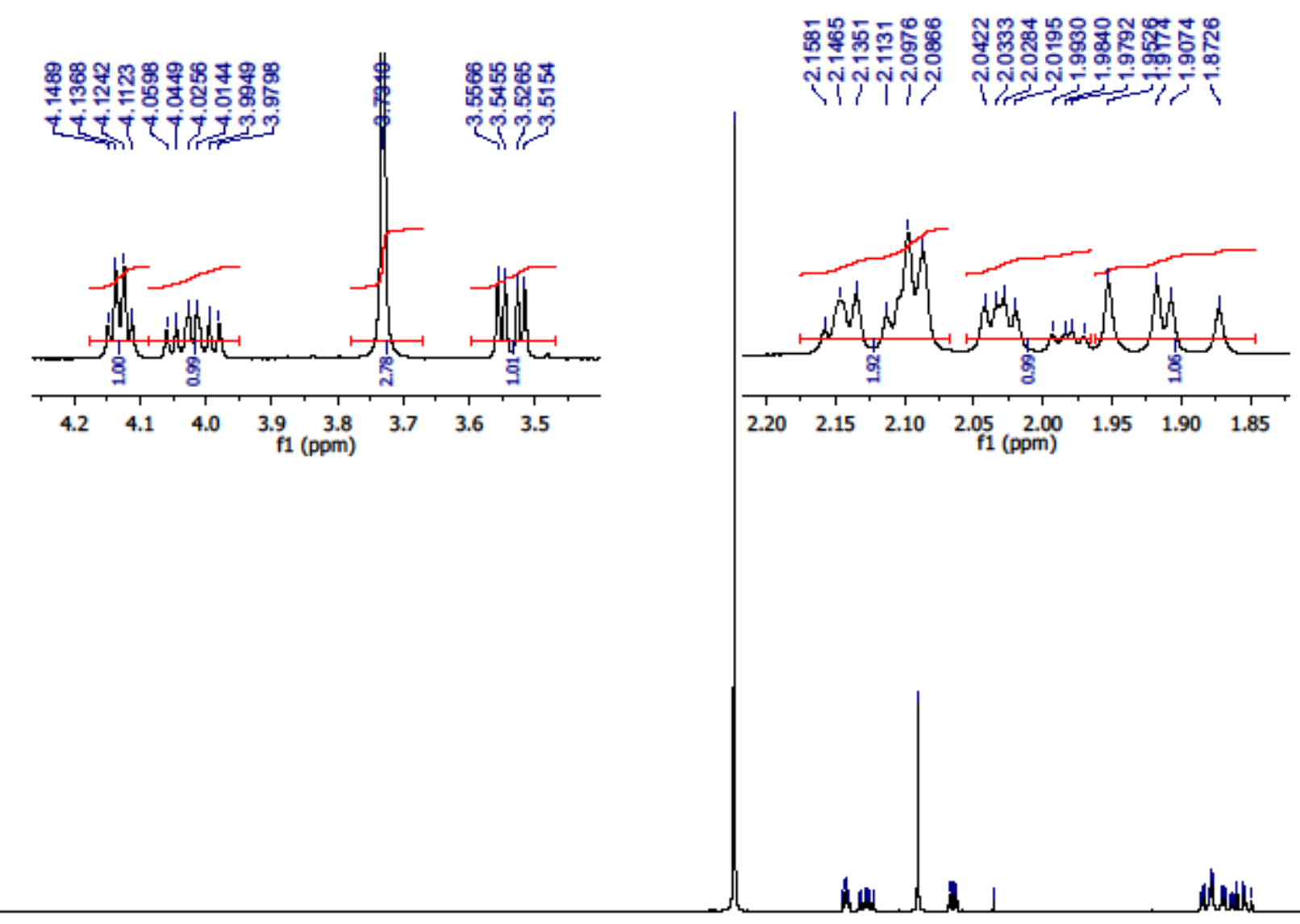

$8.5 \quad 8.0 \quad 7.5$

$7.0 \quad 6.5$

6.0

5.5

$5.0 \quad 4.5 \quad 4.0$

3.5 
Methyl (3R,5R)-1,4-dihydroxy-3,5-tert-butyldimethylsilyloxyclohexanecarboxylate Q2

${ }^{1}$ H NMR (300MHz, $\left.\mathrm{CDCl}_{3}, 293 \mathrm{~K}\right)$
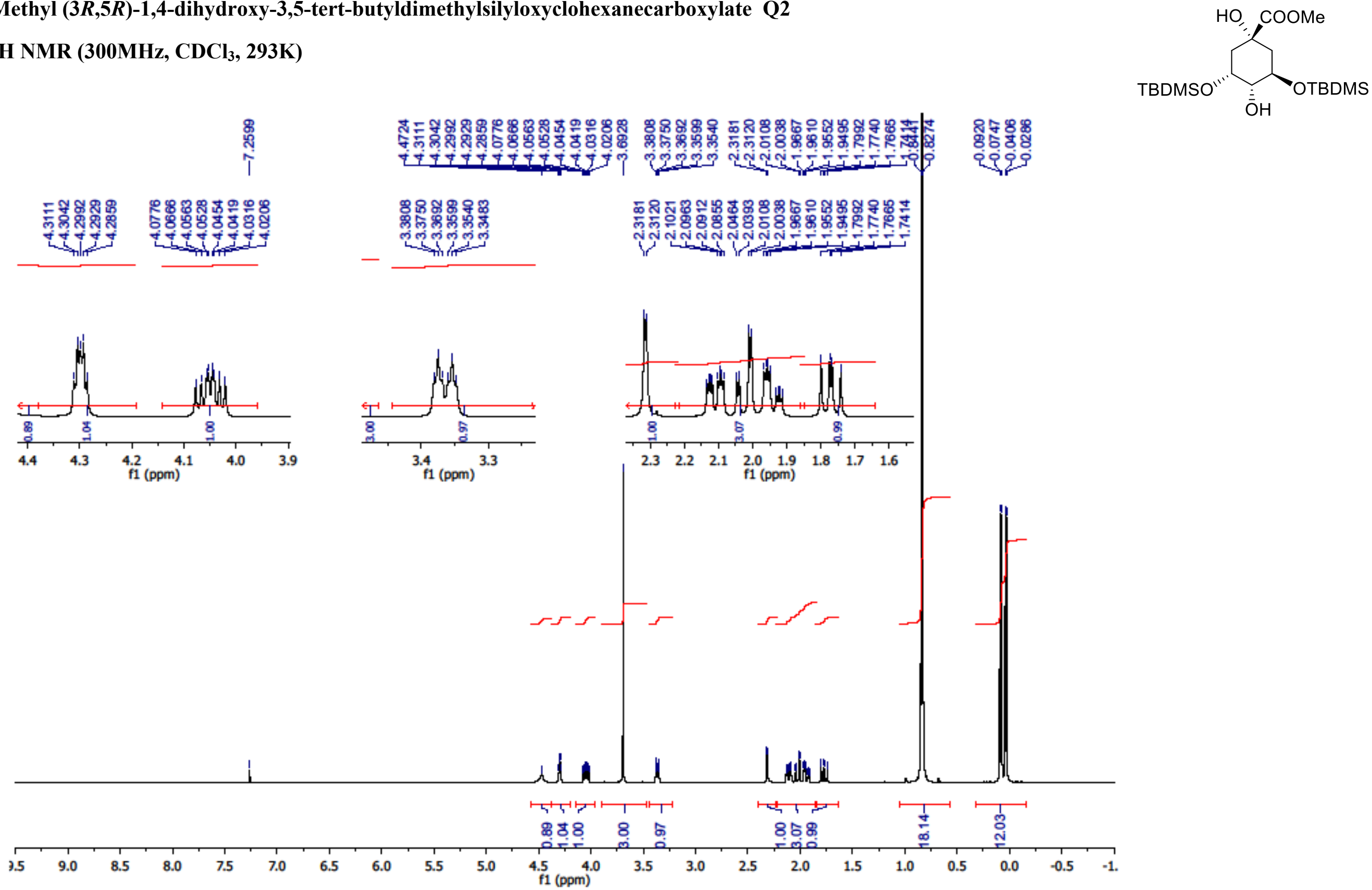
${ }^{13} \mathrm{C}$ NMR (100MHz, $\left.\mathrm{CDCl}_{3}, 293 \mathrm{~K}\right)$
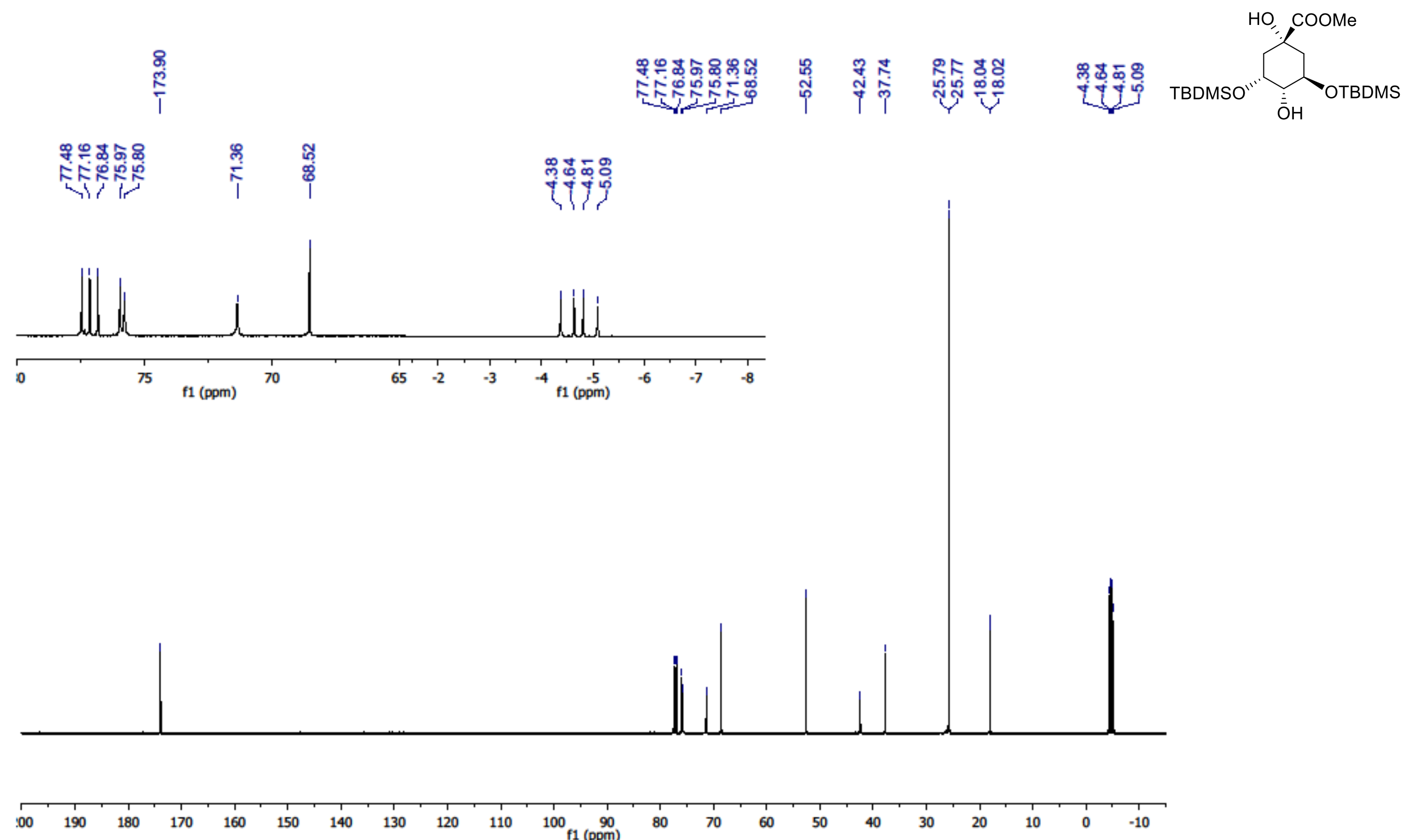
Methyl (3R,5R)-1-hydroxy-4-[(1H-imidazol-1-ylcarbothioyl)oxy]-3,5-tert-butyldimethylsilyloxy cyclohexanecarboxylate Q3

${ }^{1}$ H NMR (400MHz, $\left.\mathrm{CDCl}_{3}, 296 \mathrm{~K}\right)$
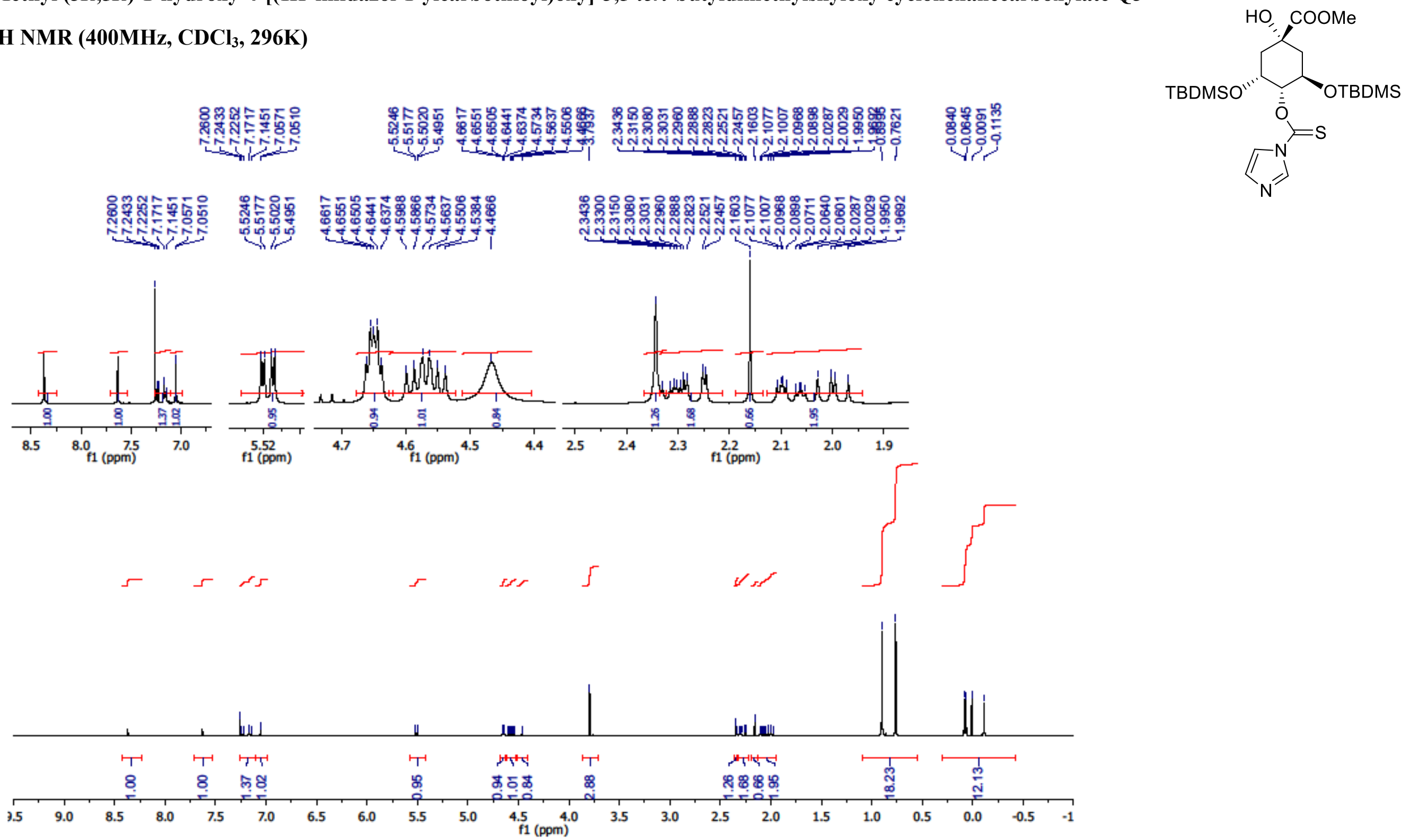
${ }^{13} \mathrm{C}$ NMR (100MHz, $\left.\mathrm{CDCl}_{3}, 297 \mathrm{~K}\right)$

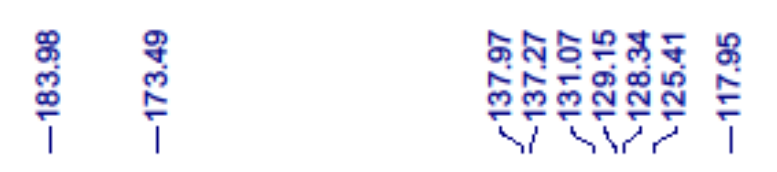

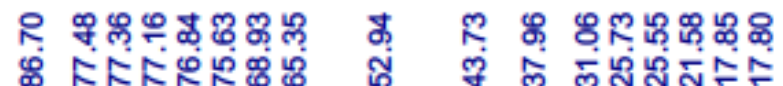

:

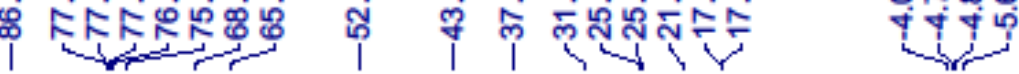
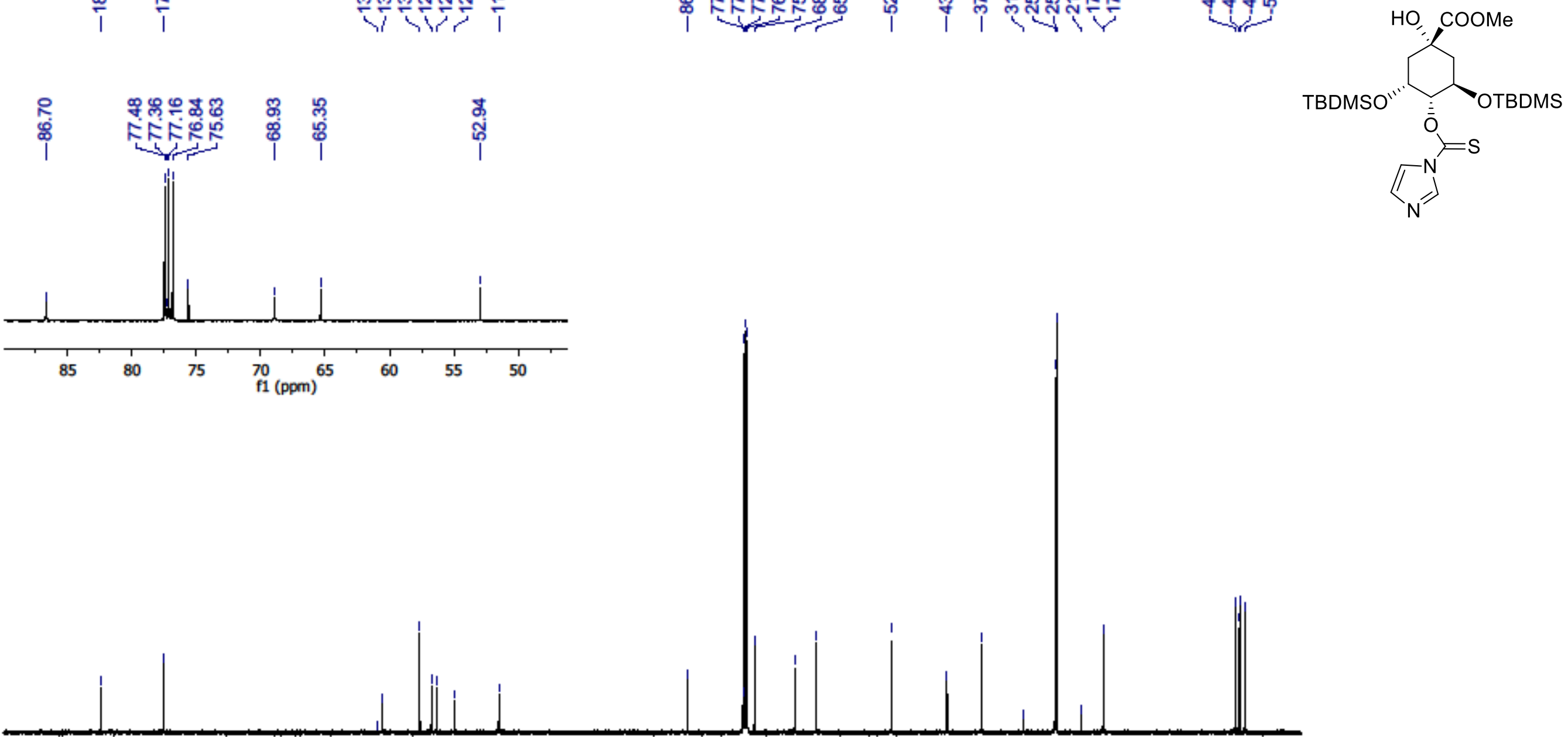

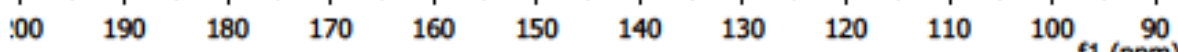


Methyl (3S,5S)-1-hydroxy-3,5- tert-butyldimethylsilyloxy cyclohexanecarboxylate 5

${ }^{1}$ H NMR (400MHz, $\left.\mathrm{CDCl}_{3}, 296 \mathrm{~K}\right)$

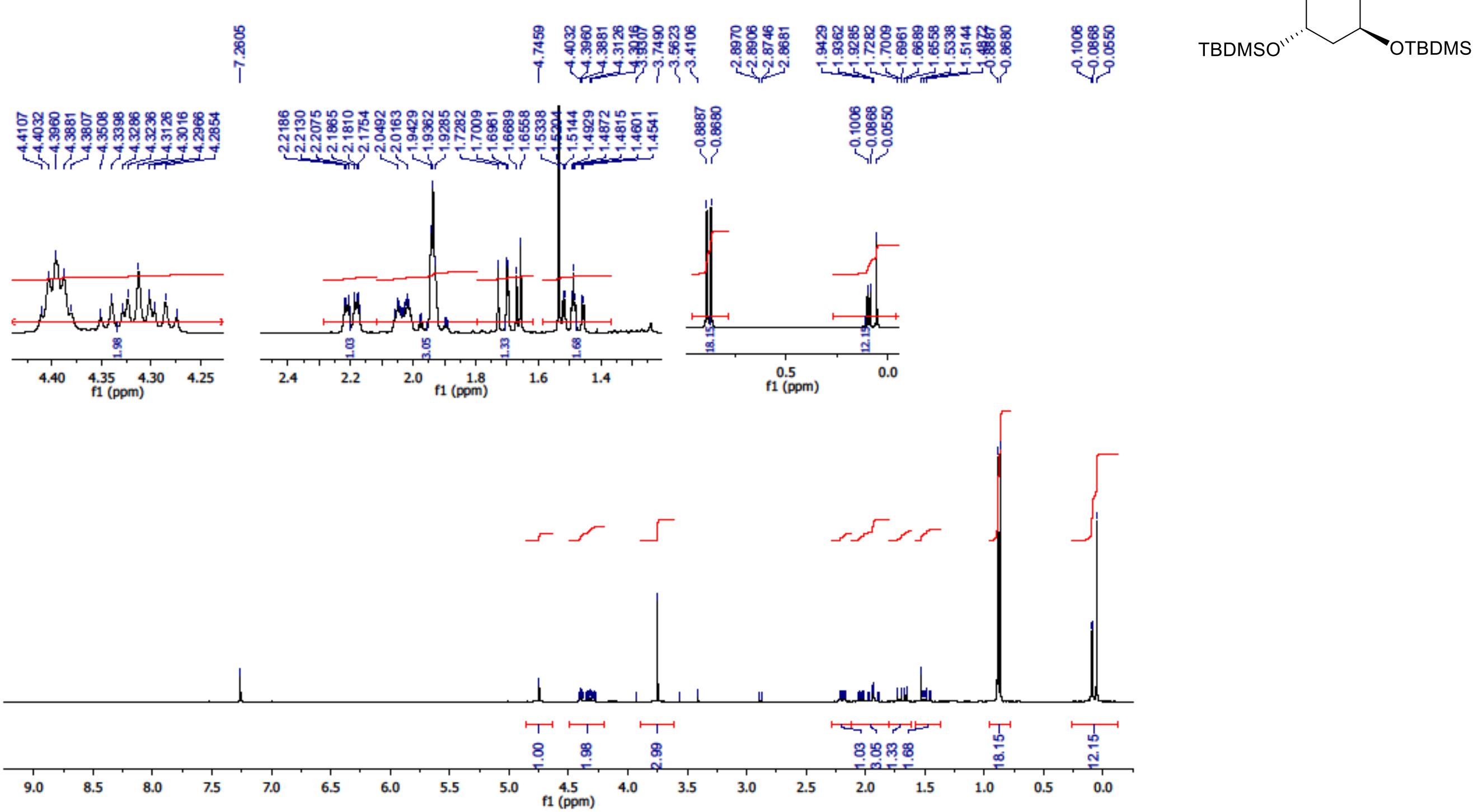


${ }^{13} \mathrm{C}$ NMR (100MHz, $\left.\mathrm{CDCl}_{3}, 297 \mathrm{~K}\right)$
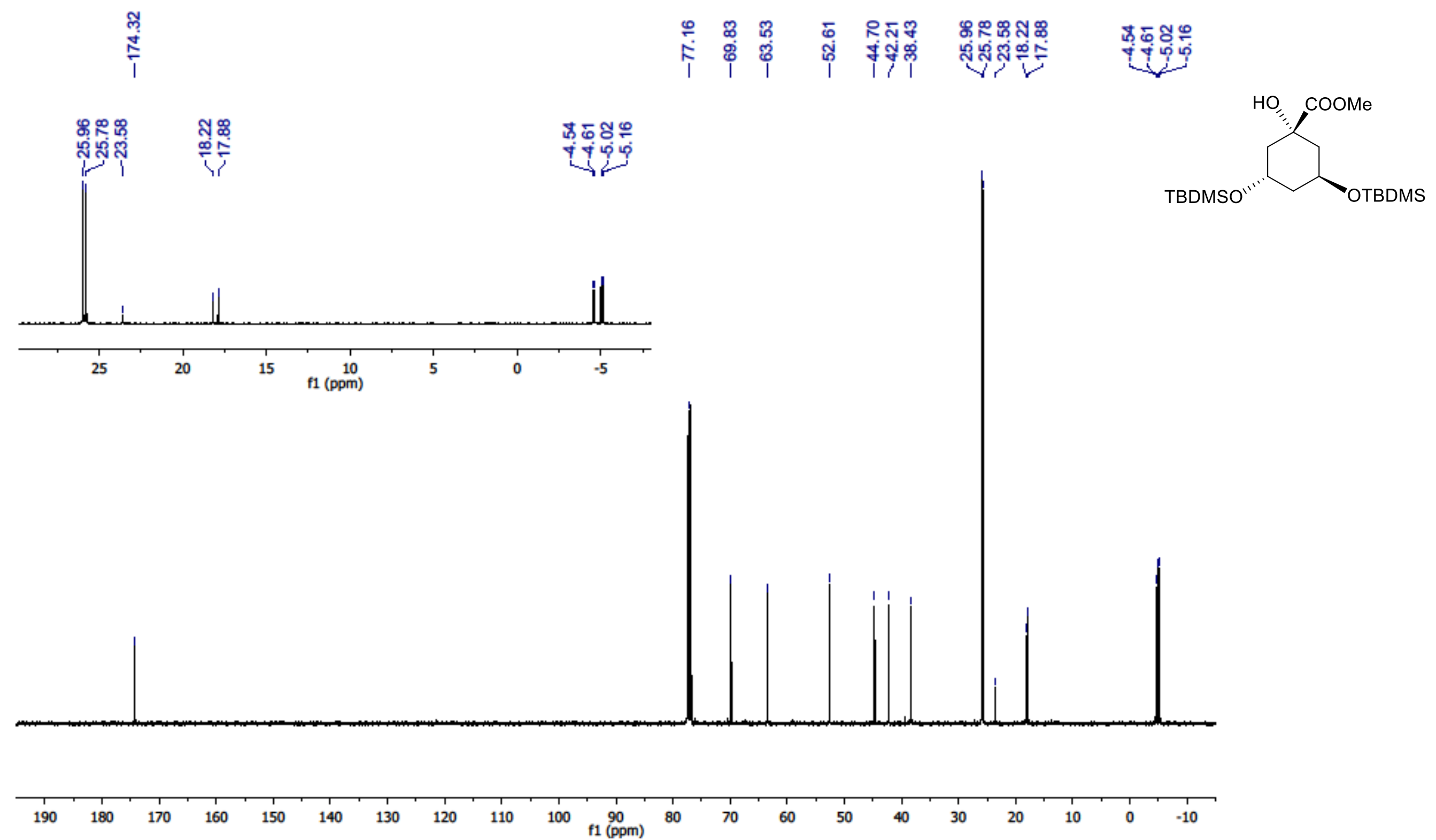
Methyl (3S,5S)-1,3,5-trihydroxy cyclohexanecarboxylate 6

${ }^{1}$ H NMR (300MHz, $\left.\mathrm{CDCl}_{3}, 296 \mathrm{~K}\right)$

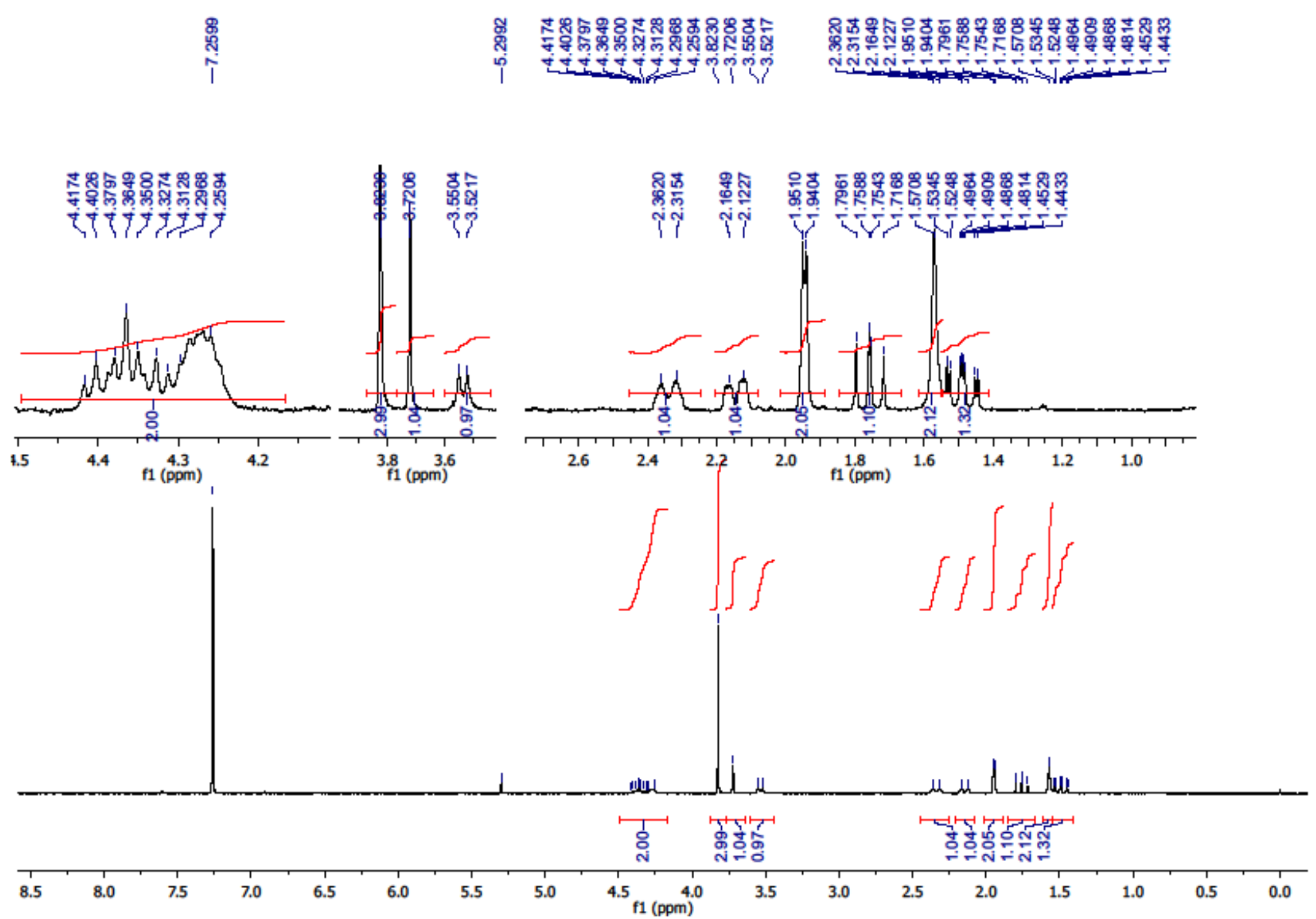


${ }^{1}$ H NMR (400MHz, $\left.\mathrm{CD}_{3} \mathrm{OD}, 296 \mathrm{~K}\right)$
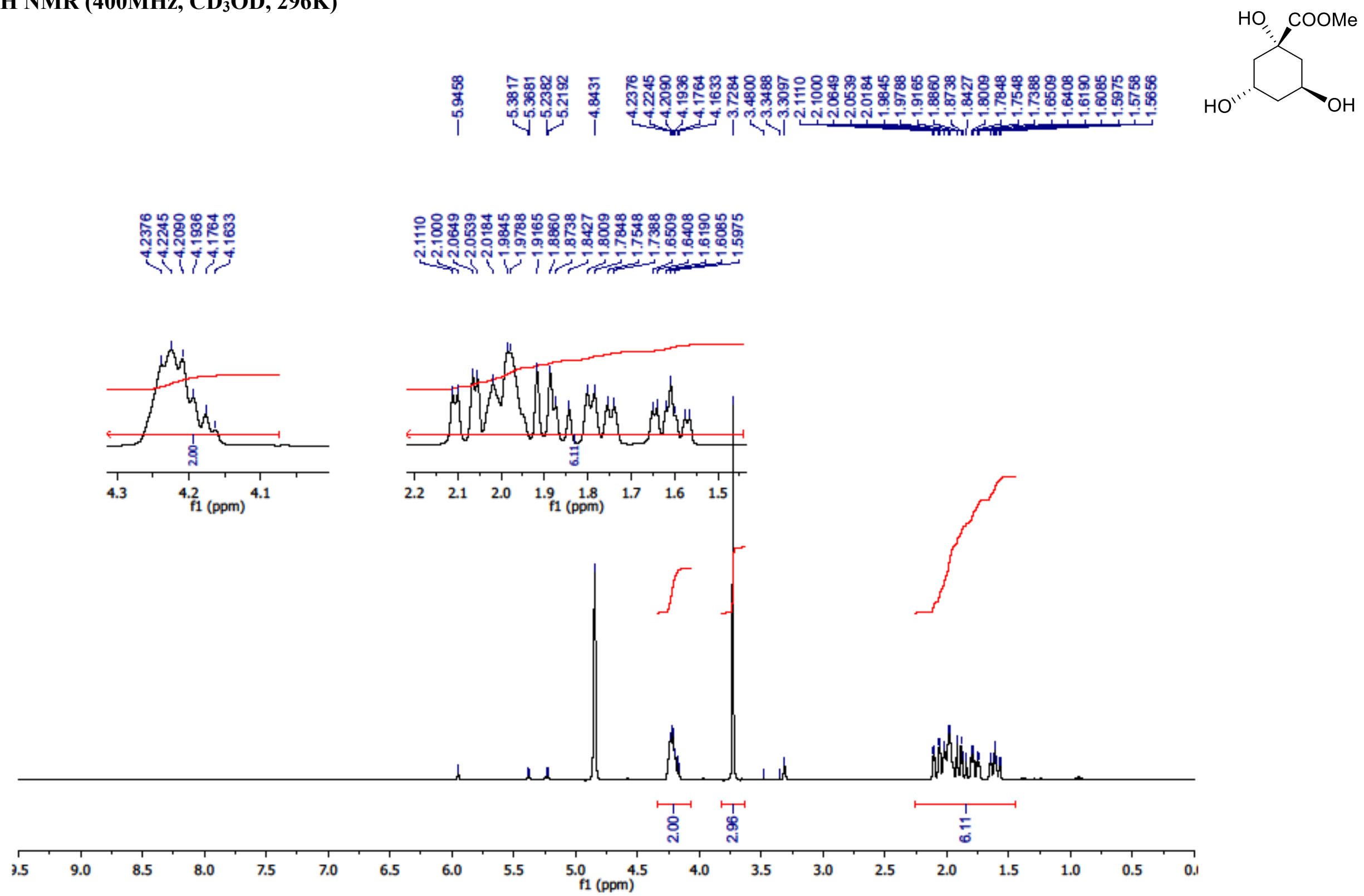
${ }^{13} \mathrm{C}$ NMR (100MHz, CD $\left.{ }_{3} \mathrm{OD}, 295 \mathrm{~K}\right)$

$\underset{\frac{1}{i}}{\frac{0}{1}}$

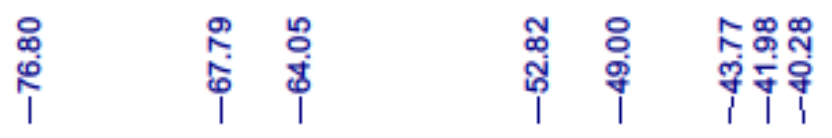
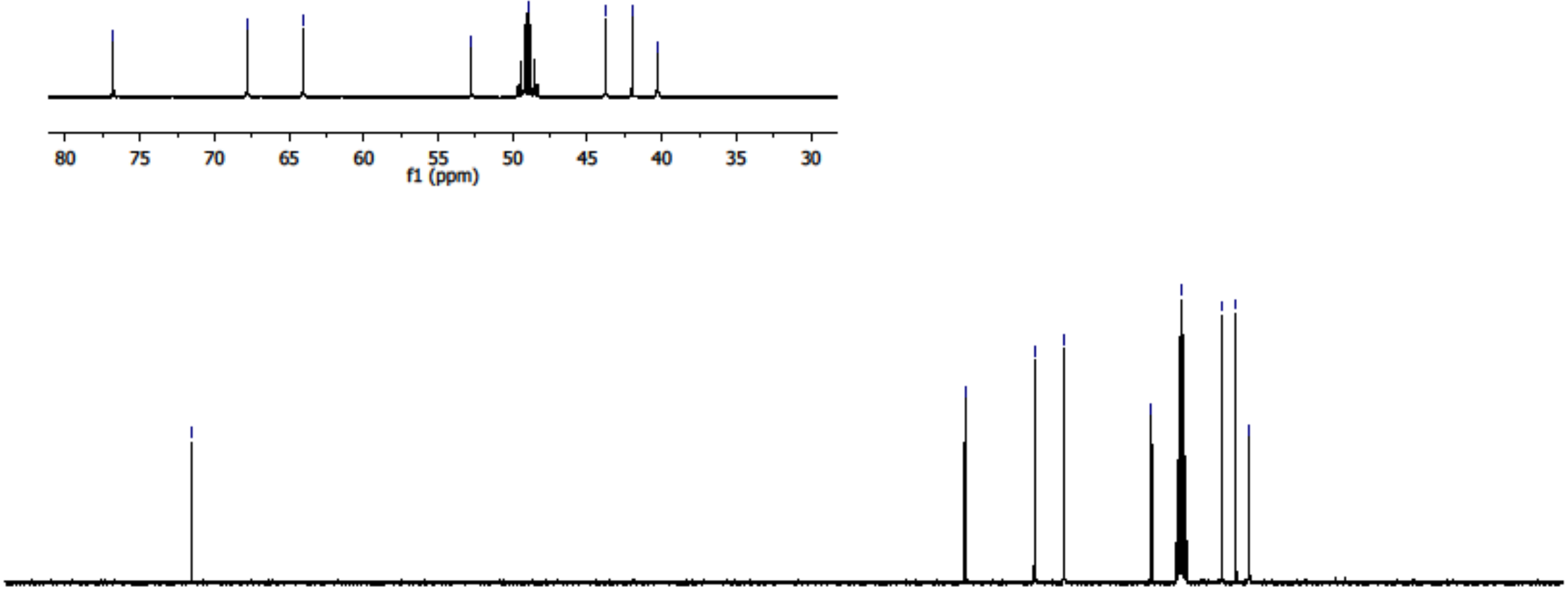
Methyl (3S,5S) 1-hydroxy-3,5 bis[[(2E)-3-(3,4-diacetoxyphenyl)-1-oxo-2-propen-1-yl]oxy]cyclohexanecarboxylate 9a

${ }^{1} \mathrm{H}$ NMR (500MHz, $\left.\mathrm{CDCl}_{3}, 297 \mathrm{~K}\right)$

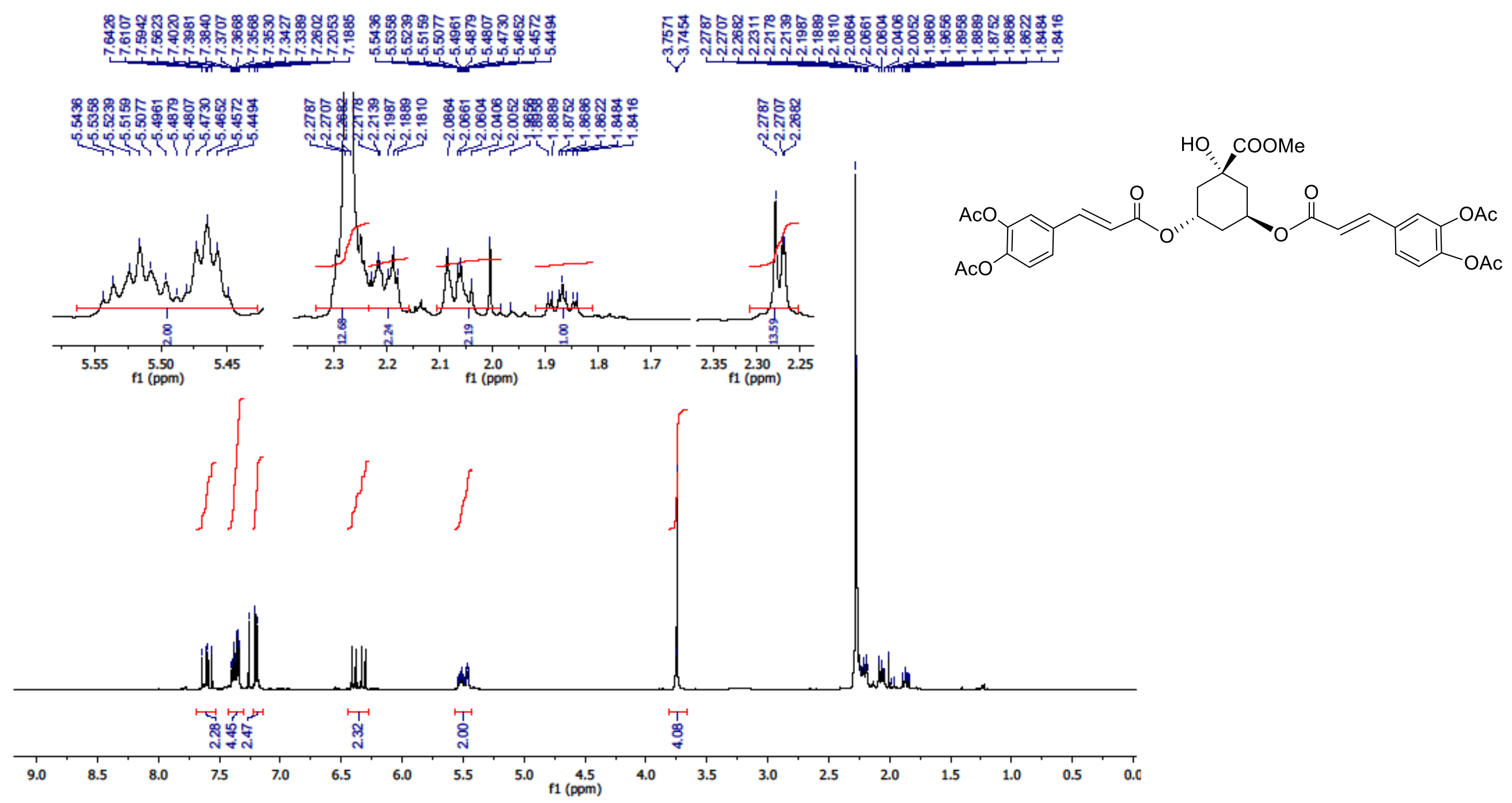


${ }^{13} \mathrm{C}$ NMR (125MHz, CD $\left.{ }_{3} \mathrm{OD}, 295 \mathrm{~K}\right)$
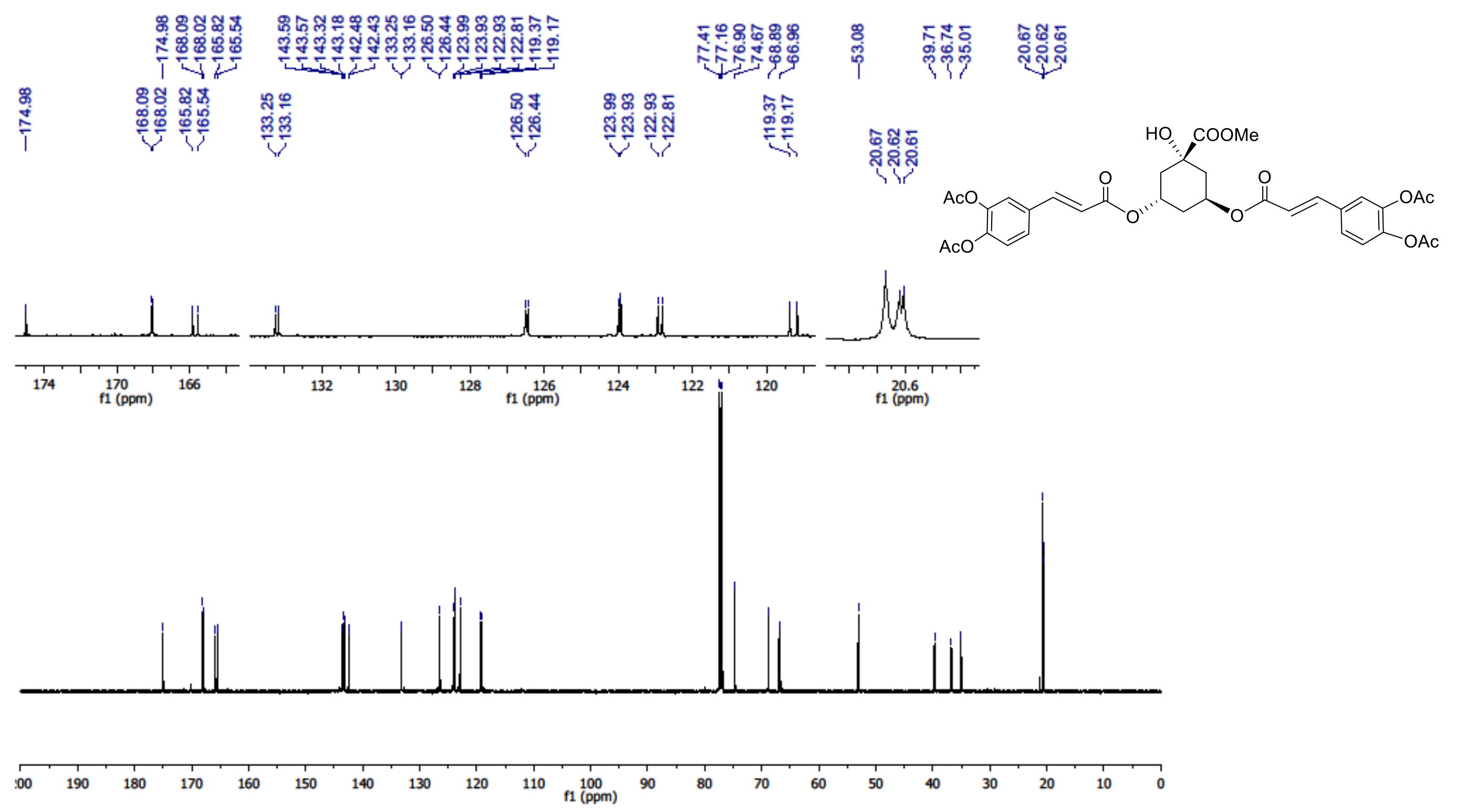
Methyl (3S,5S) 1-hydroxy-3,5 bis[[(2E)-3-(3,4-di-( prop-2-en-1-yloxy)phenyl)-1-oxo-2-propen-1-yl]oxy]cyclohexanecarboxylate 9b

${ }^{1}$ H NMR (300MHz, $\left.\mathrm{CDCl}_{3}, 296 \mathrm{~K}\right)$

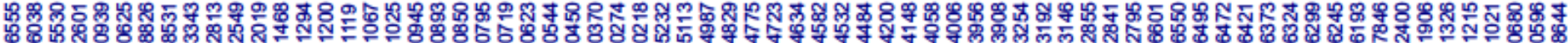

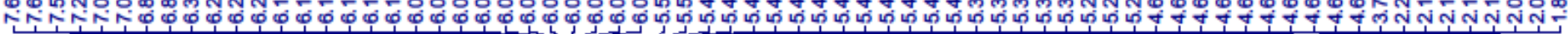

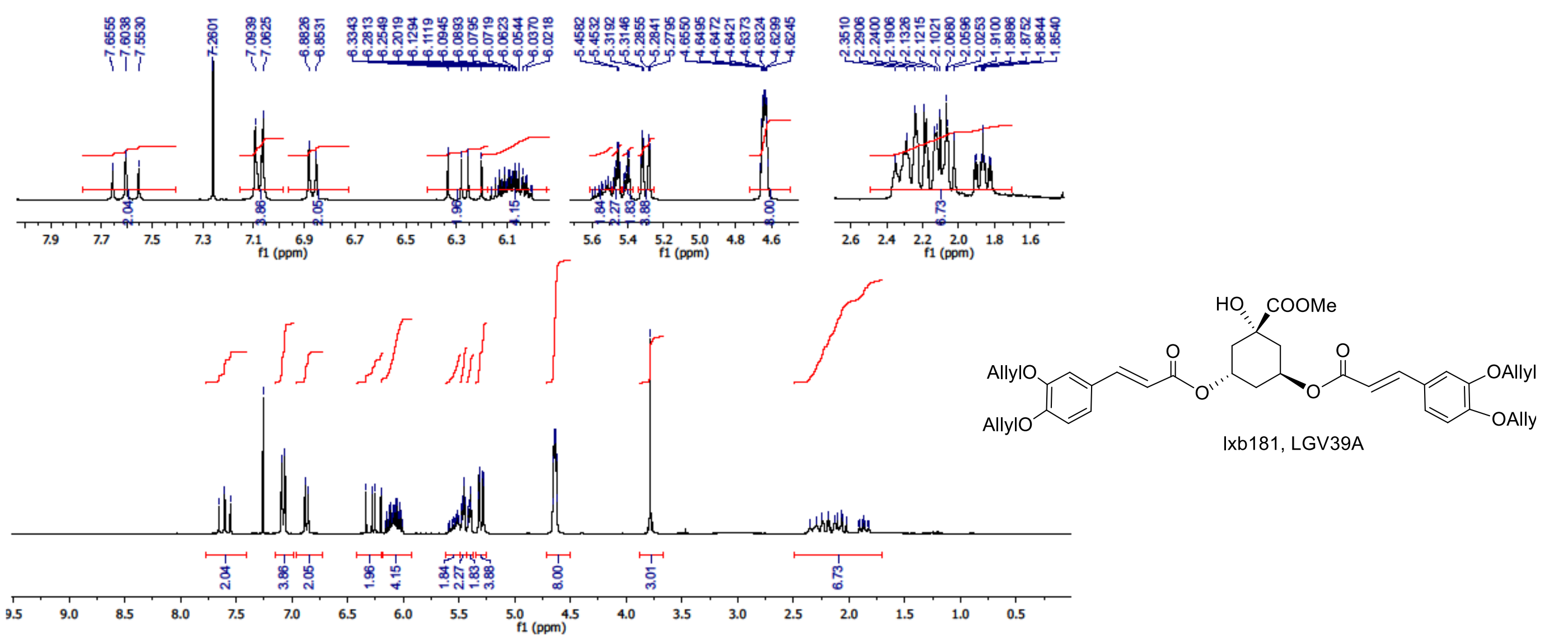




\section{${ }^{13} \mathrm{C}$ NMR (75MHz, $\left.\mathrm{CDCl}_{3}, 296 \mathrm{~K}\right)$}

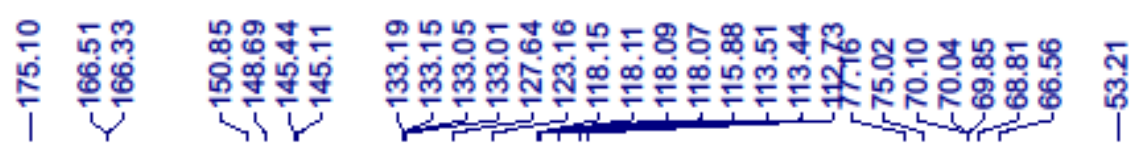

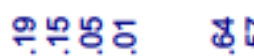

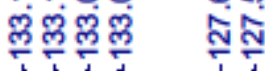

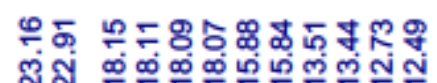

สู่สุ่

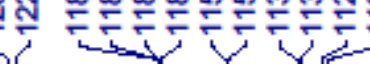

\section{우훙}

र्री

$\bar{\infty}$

\% $\stackrel{8}{\$}$

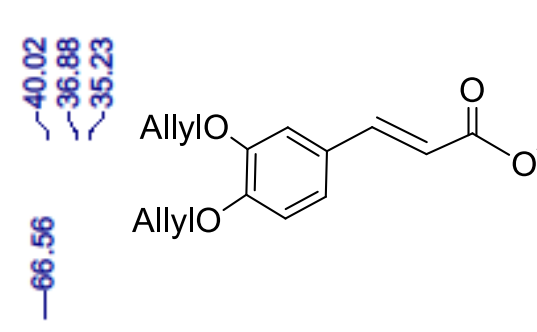

HO, cOOMe

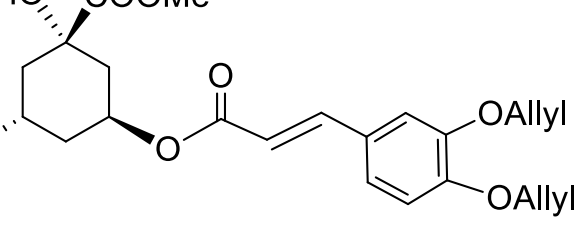

Ixb181, LGV39A
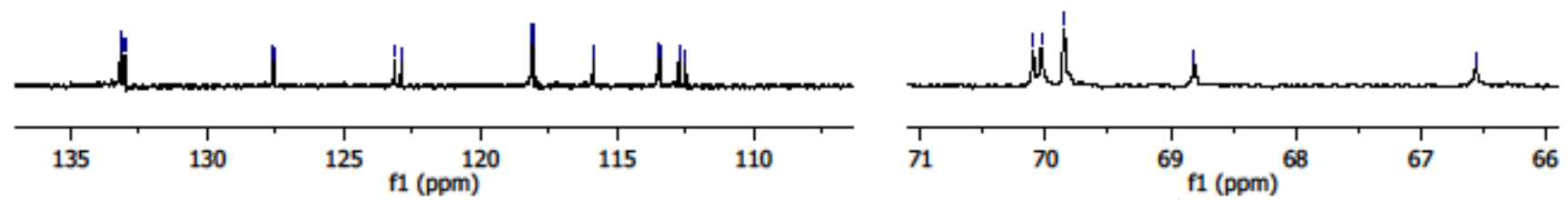

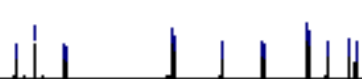

$\begin{array}{llllllllllll}210 & 200 & 190 & 180 & 170 & 160 & 150 & 140 & 130 & 120 & 110 & 100 \\ \mathrm{f} 1(\mathrm{ppm})\end{array}$

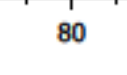

70
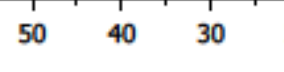
Methyl (3S,5S) 1-hydroxy-3,5 bis[[(2E)-3-(3-acetoxy,4-methoxyphenyl)-1-oxo-2-propen-1-yl]oxy]cyclohexanecarboxylate 9c

${ }^{1}$ H NMR (300MHz, $\left.\mathrm{CDCl}_{3}, 296 \mathrm{~K}\right)$

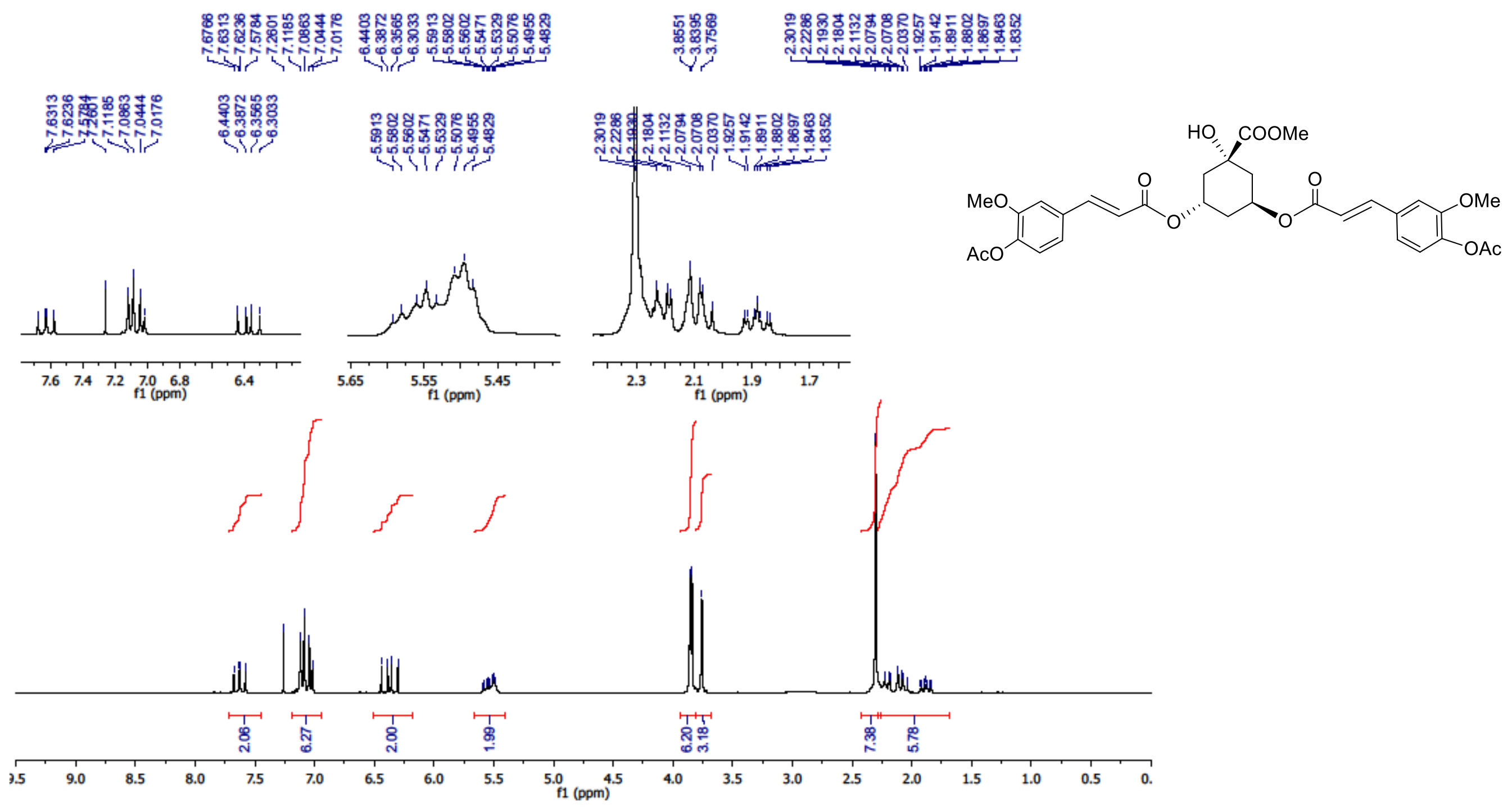


${ }^{13} \mathrm{C}$ NMR (100MHz, $\left.\mathrm{CDCl}_{3}, 295 \mathrm{~K}\right)$

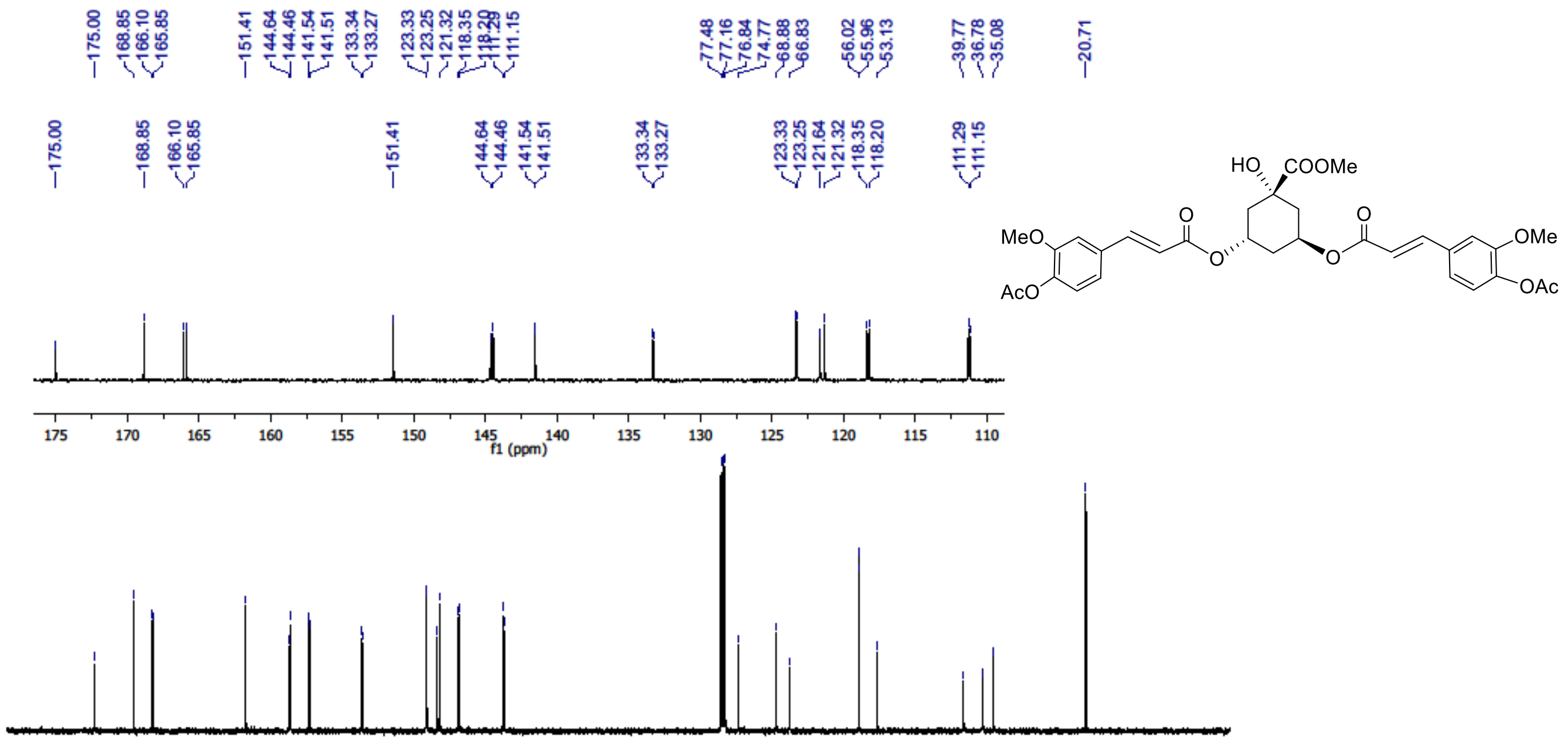


Methyl (3S,5S) 1-hydroxy-3,5 bis[[(2E)-3-(4-acetoxyphenyl)-1-oxo-2-propen-1-yl]oxy]cyclohexanecarboxylate 9d

${ }^{1}$ H NMR (300MHz, $\left.\mathrm{CDCl}_{3}, 296 \mathrm{~K}\right)$
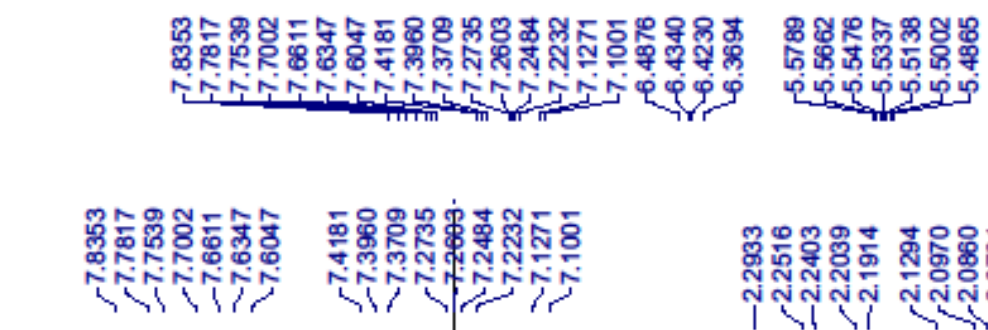

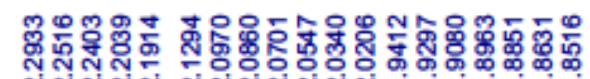
กู่ง
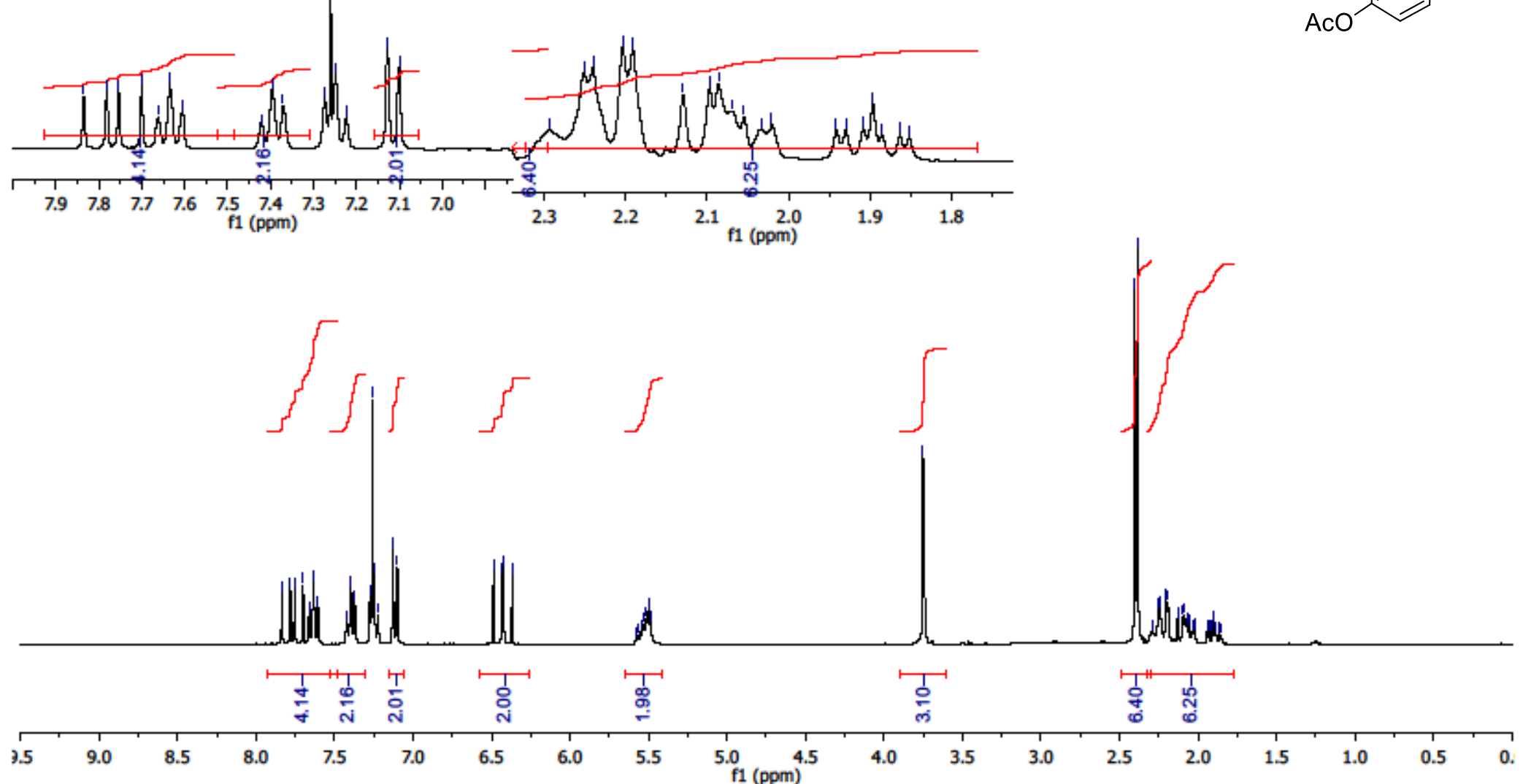

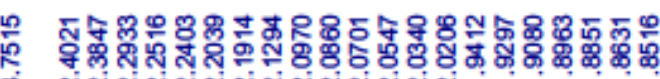

1 N่ง

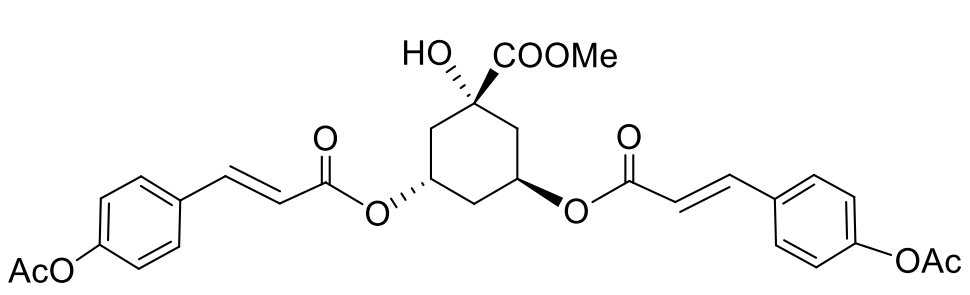


${ }^{13} \mathrm{C}$ NMR (100MHz, $\left.\mathrm{CDCl}_{3}, 295 \mathrm{~K}\right)$

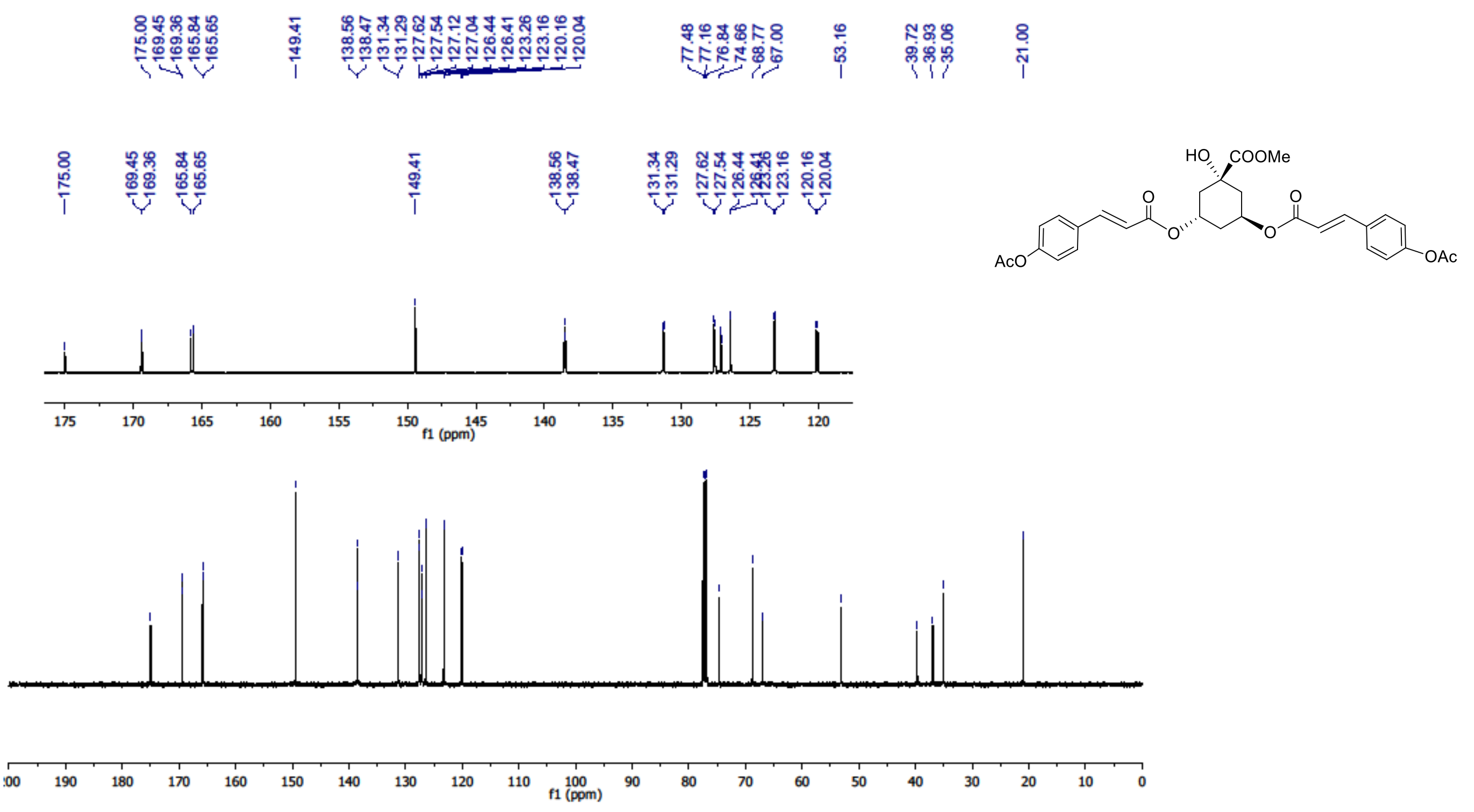


Methyl (3S,5S) 1-hydroxy-3,5 bis[[(2E)-3-(4-acetoxyphenyl)-1-oxo-2-propen-1-yl]oxy]cyclohexanecarboxylate 9e

${ }^{1} \mathrm{H}$ NMR (400MHz, $\left.\mathrm{CDCl}_{3}, 295 \mathrm{~K}\right)$
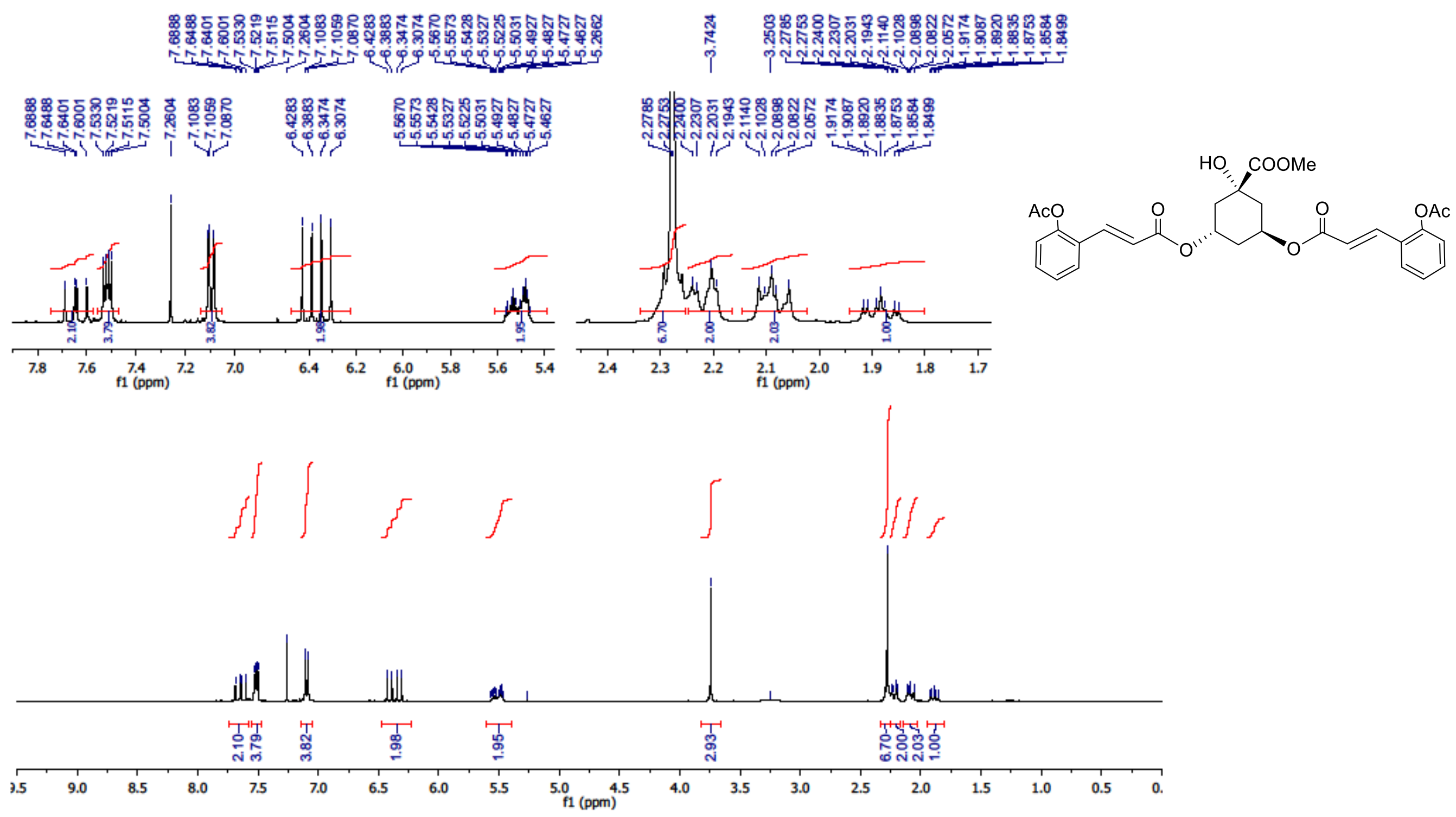
${ }^{13} \mathrm{C}$ NMR (100MHz, $\left.\mathrm{CD}_{3} \mathrm{OD}, 295 \mathrm{~K}\right)$
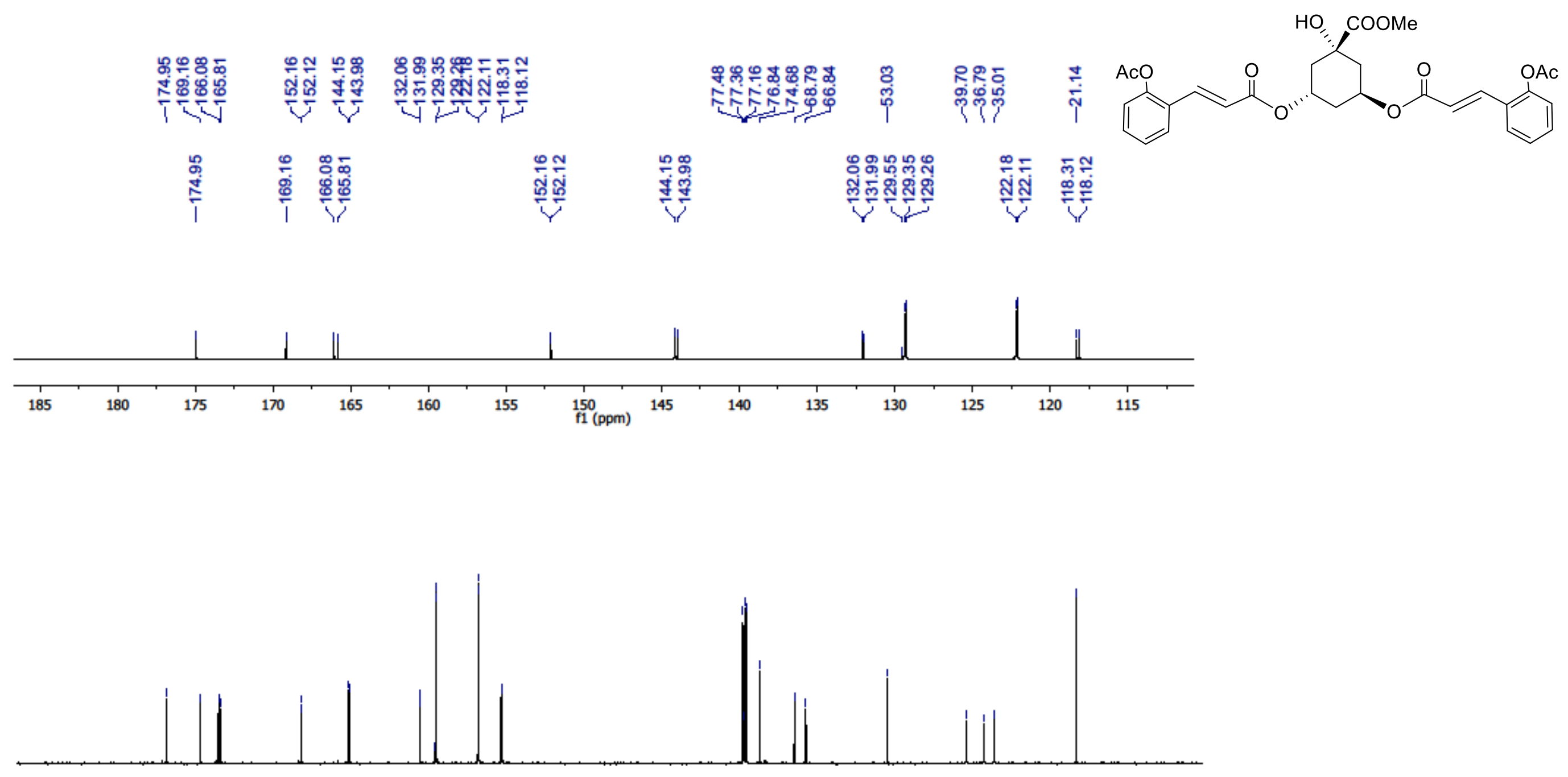

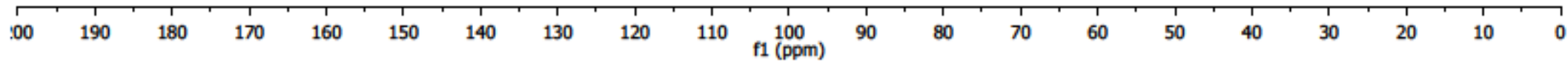


Methyl (3S,5S) 1-hydroxy-3,5 bis[[(2E)-3-(3,4-dihydroxyphenyl)-1-oxo-2-propen-1-yl]oxy]cyclohexanecarboxylate 10a

${ }^{1} \mathrm{H}$ NMR (500MHz, $\left.\mathrm{CD}_{3} \mathrm{OD}, 298 \mathrm{~K}\right)$

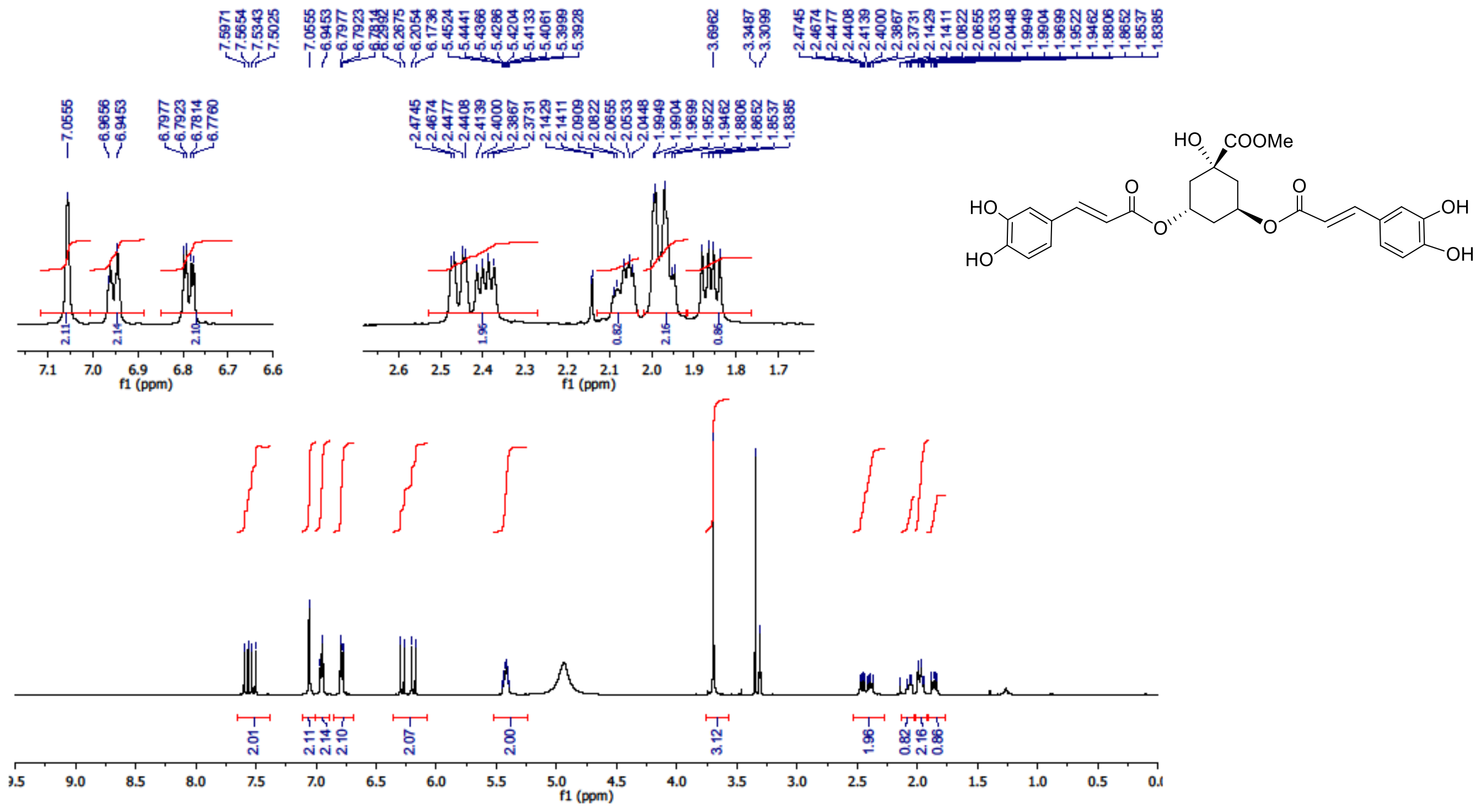


${ }^{13} \mathrm{C}$ NMR (125MHz, CD $\left.\mathrm{OD}, 297 \mathrm{~K}\right)$
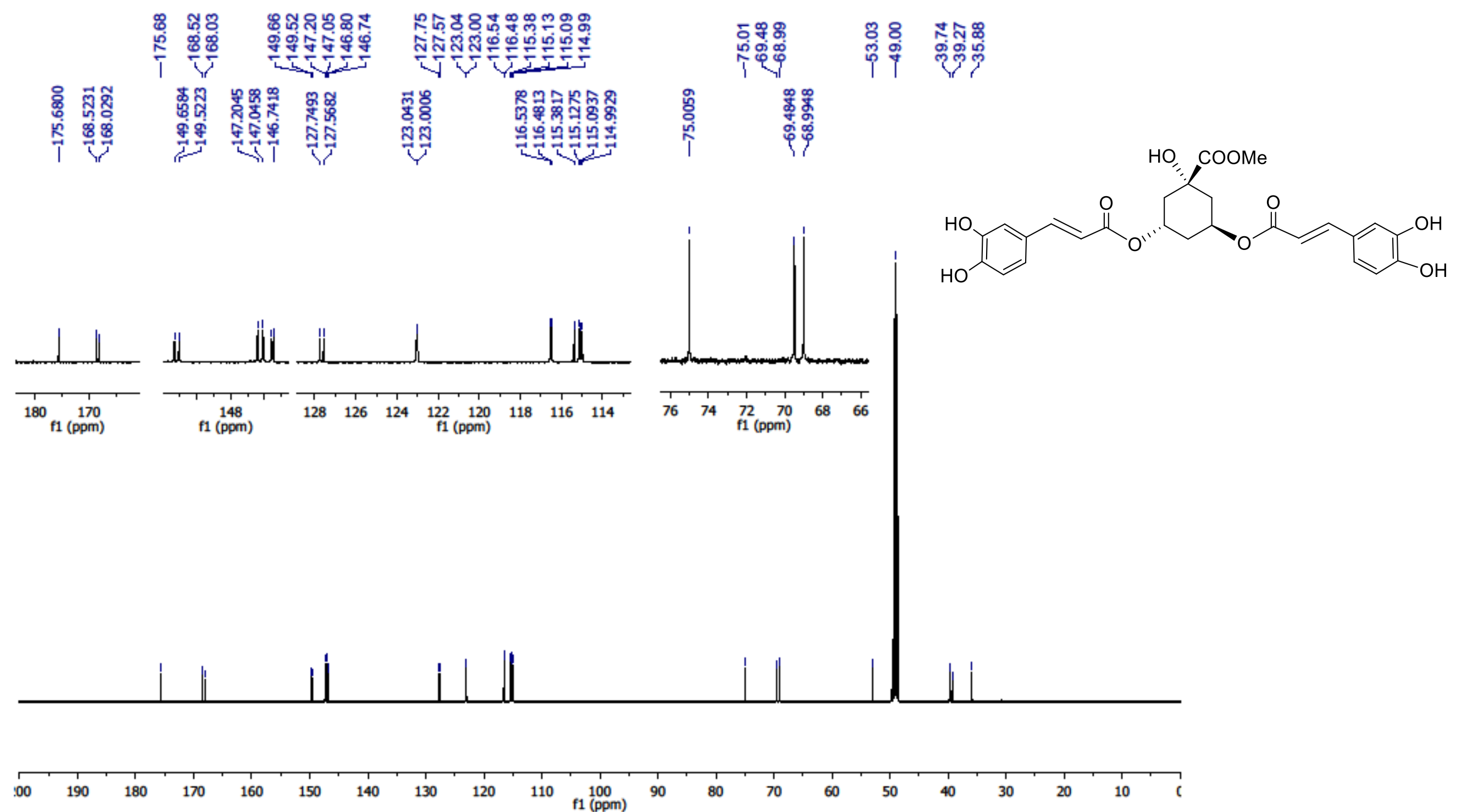
Methyl (3S,5S) 1-hydroxy-3,5 bis[[(2E)- 3-(4-hydroxy-3-methoxyphenyl)-1-oxo-2-propen-1-yl]oxy]cyclohexanecarboxylate 10c

${ }^{1}$ H NMR (400MHz, $\left.\mathrm{CD}_{3} \mathrm{OD}, 295 \mathrm{~K}\right)$
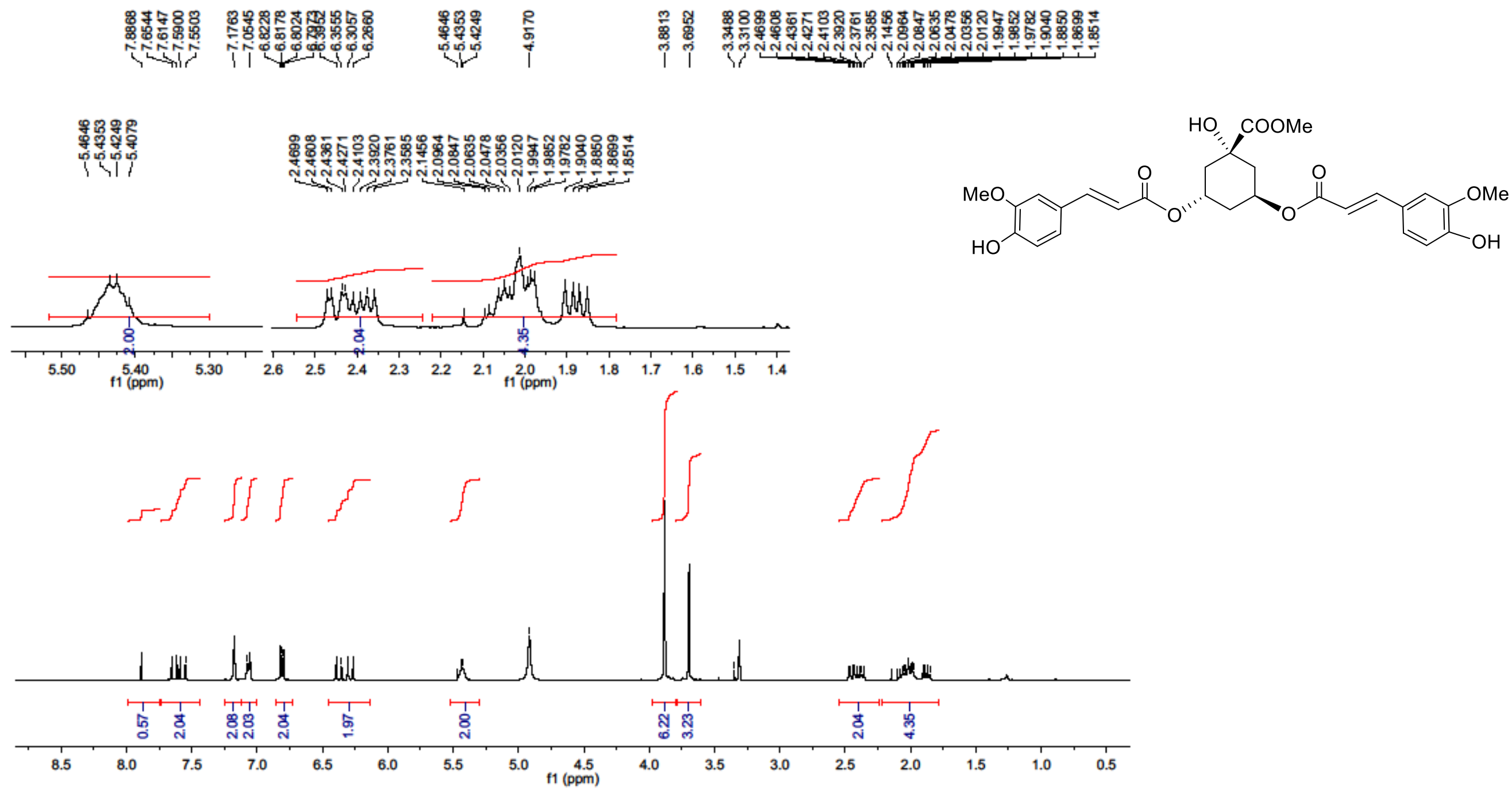
${ }^{13} \mathrm{C}$ NMR (100MHz, CD $\left.{ }_{3} \mathrm{OD}, 295 \mathrm{~K}\right)$

\begin{tabular}{|c|c|c|c|c|}
\hline 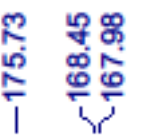 & 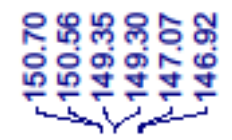 & 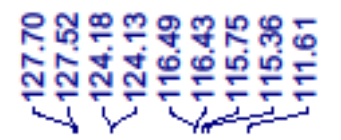 & 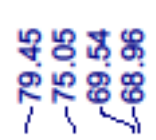 & 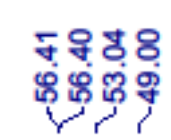 \\
\hline
\end{tabular}
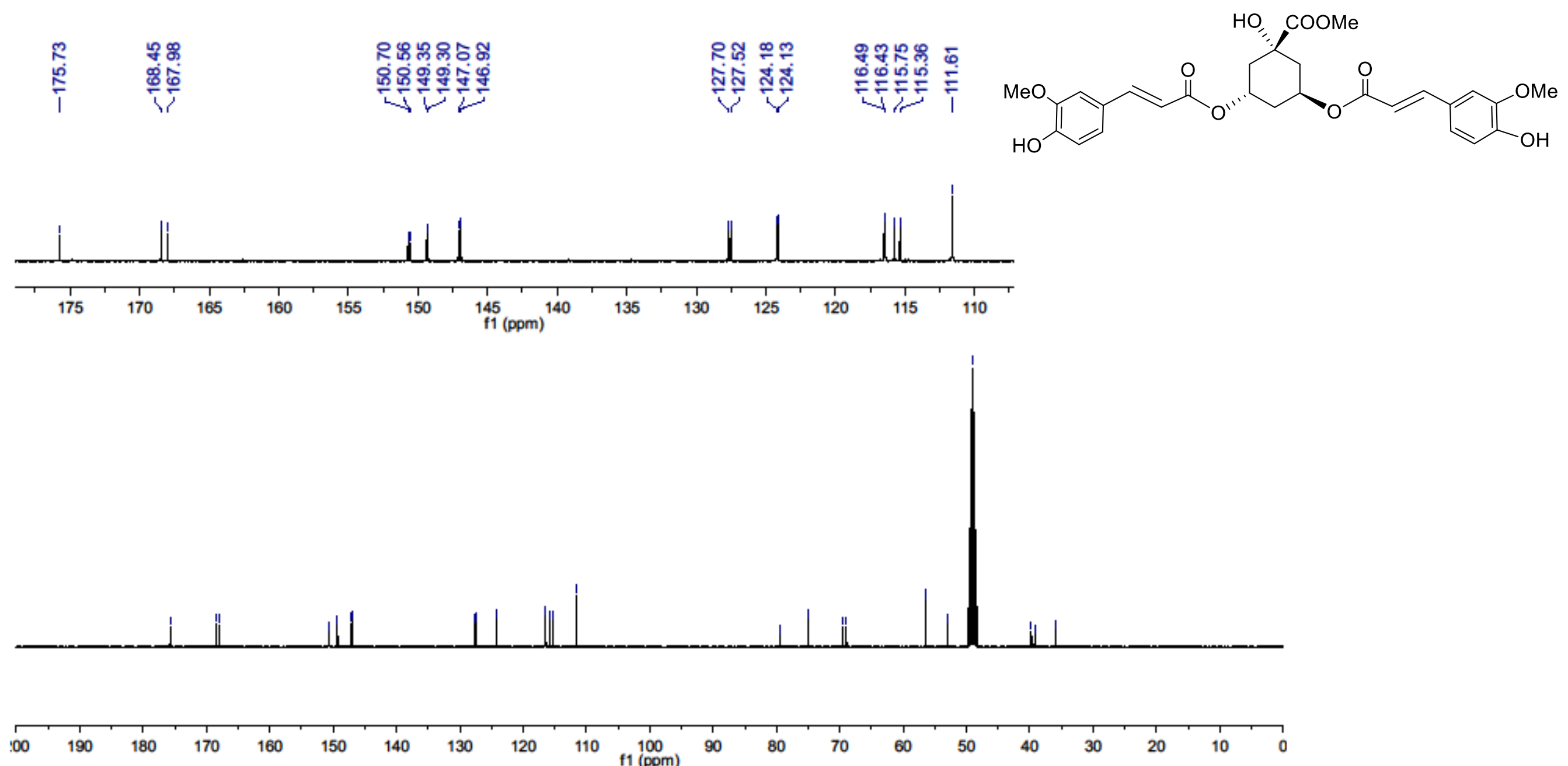
Methyl (3S,5S) 1-hydroxy-3,5 bis[[(2E)-3-(4-hydroxyphenyl)-1-oxo-2-propen-1-yl]oxy]cyclohexanecarboxylate 10d

${ }^{1}$ H NMR (400MHz, $\left.\mathrm{CD}_{3} \mathrm{OD}, 295 \mathrm{~K}\right)$

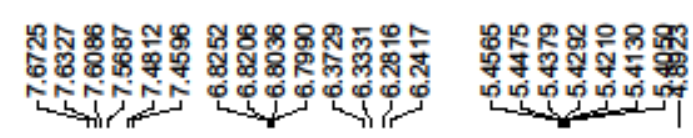

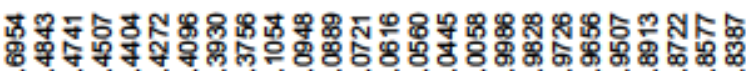

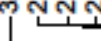

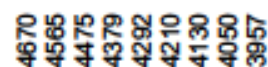

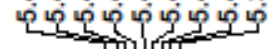

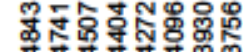

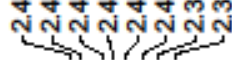

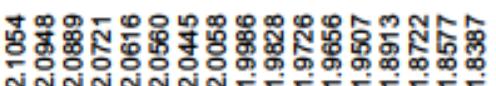

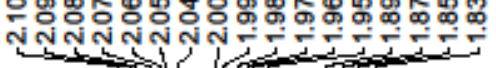
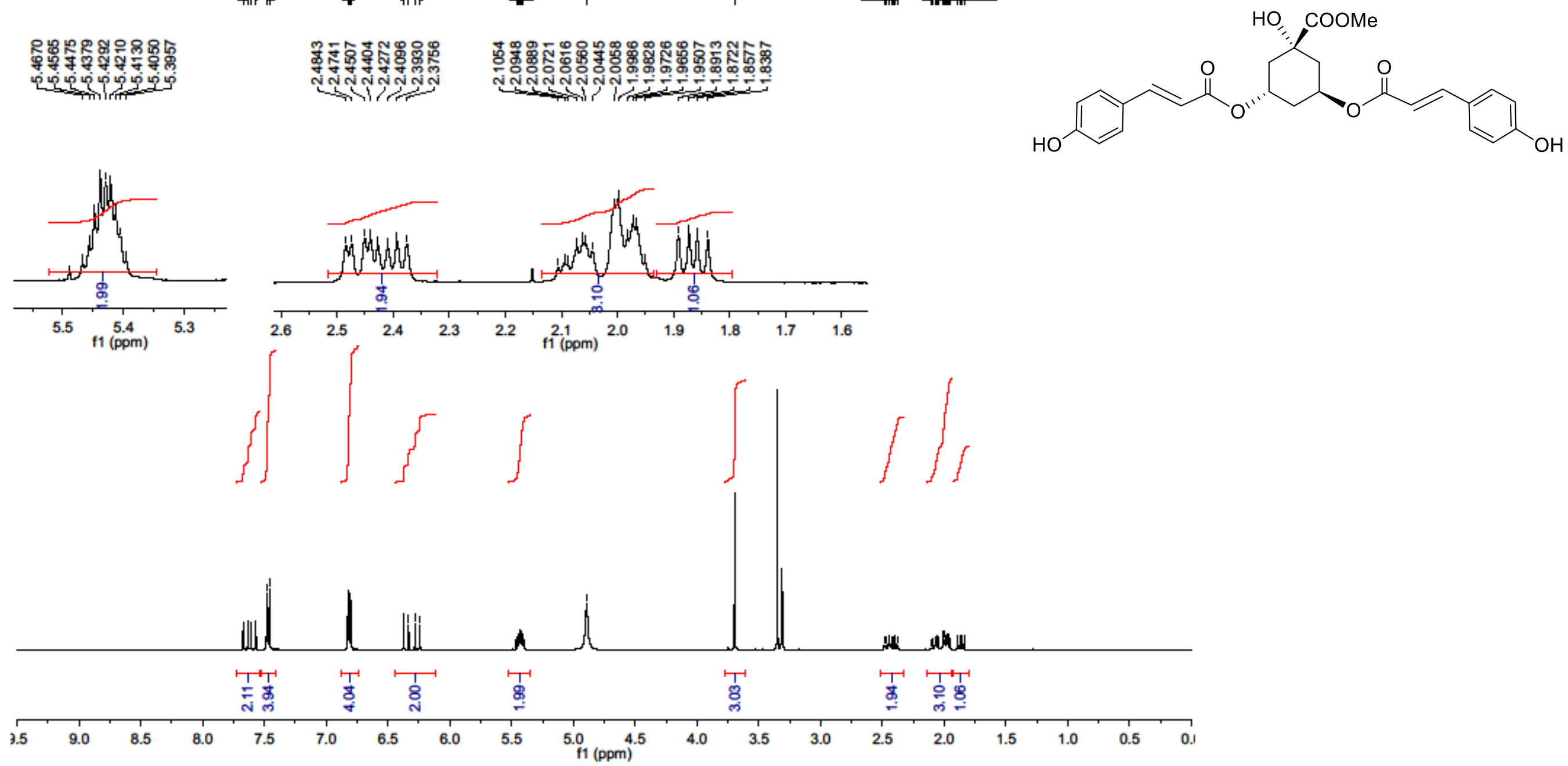
${ }^{13} \mathrm{C}$ NMR (100MHz, CD $\left.\mathrm{OD}, 295 \mathrm{~K}\right)$

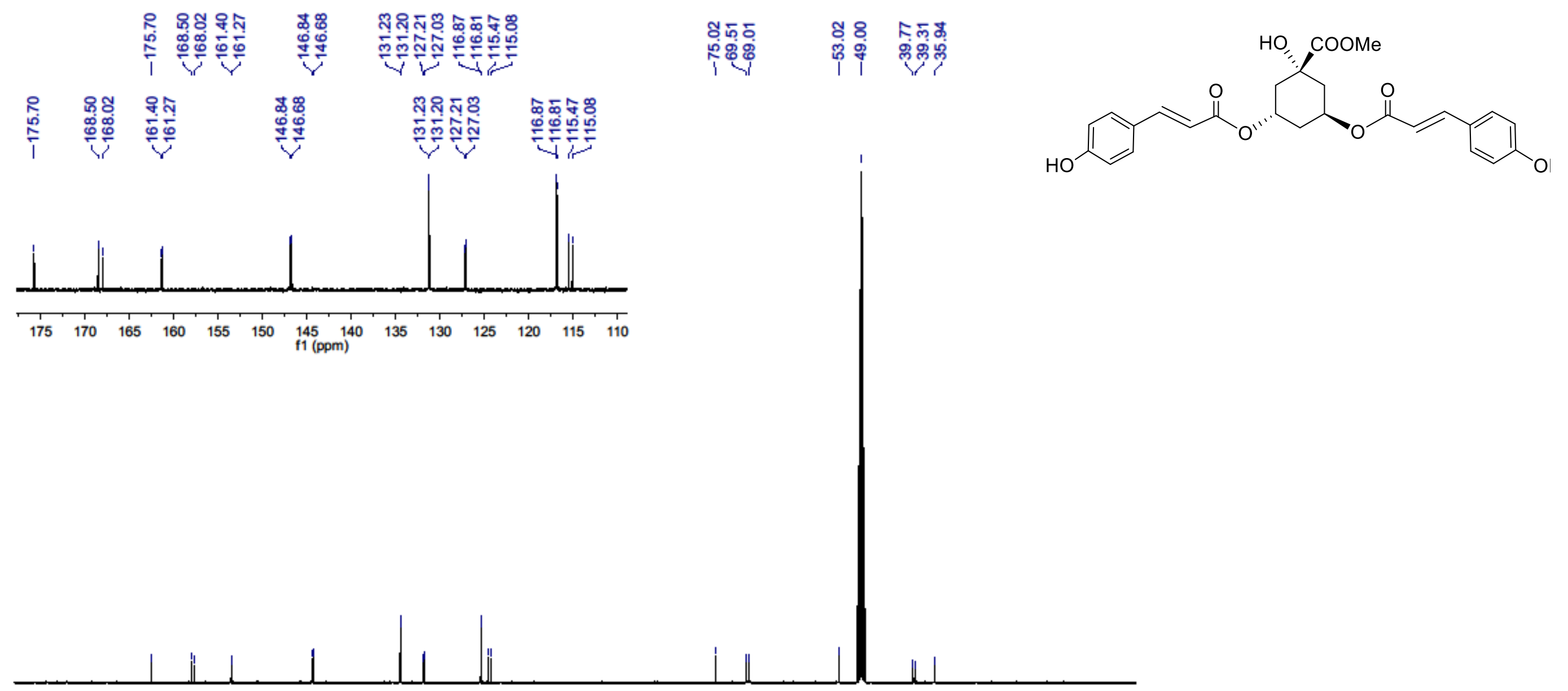


Methyl (3S,5S) 1-hydroxy-3,5 bis[[(2E)-3-(2-hydroxyphenyl)-1-oxo-2-propen-1-yl]oxy]cyclohexanecarboxylate 10e

${ }^{1}$ H NMR (400MHz, CD $\mathrm{CD}_{3}$, 295K)
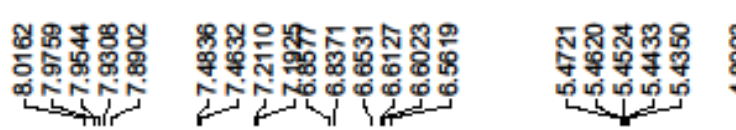

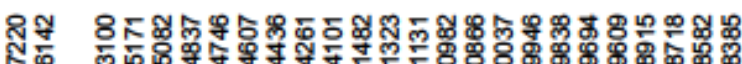

证证

i

欷

\begin{tabular}{|c|c|c|c|c|c|}
\hline & 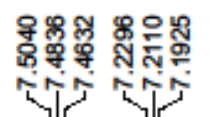 & 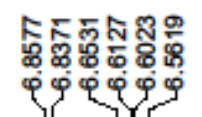 & 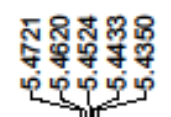 & 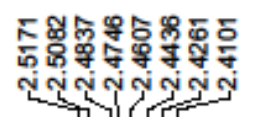 & 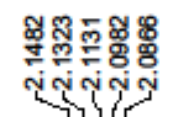 \\
\hline
\end{tabular}
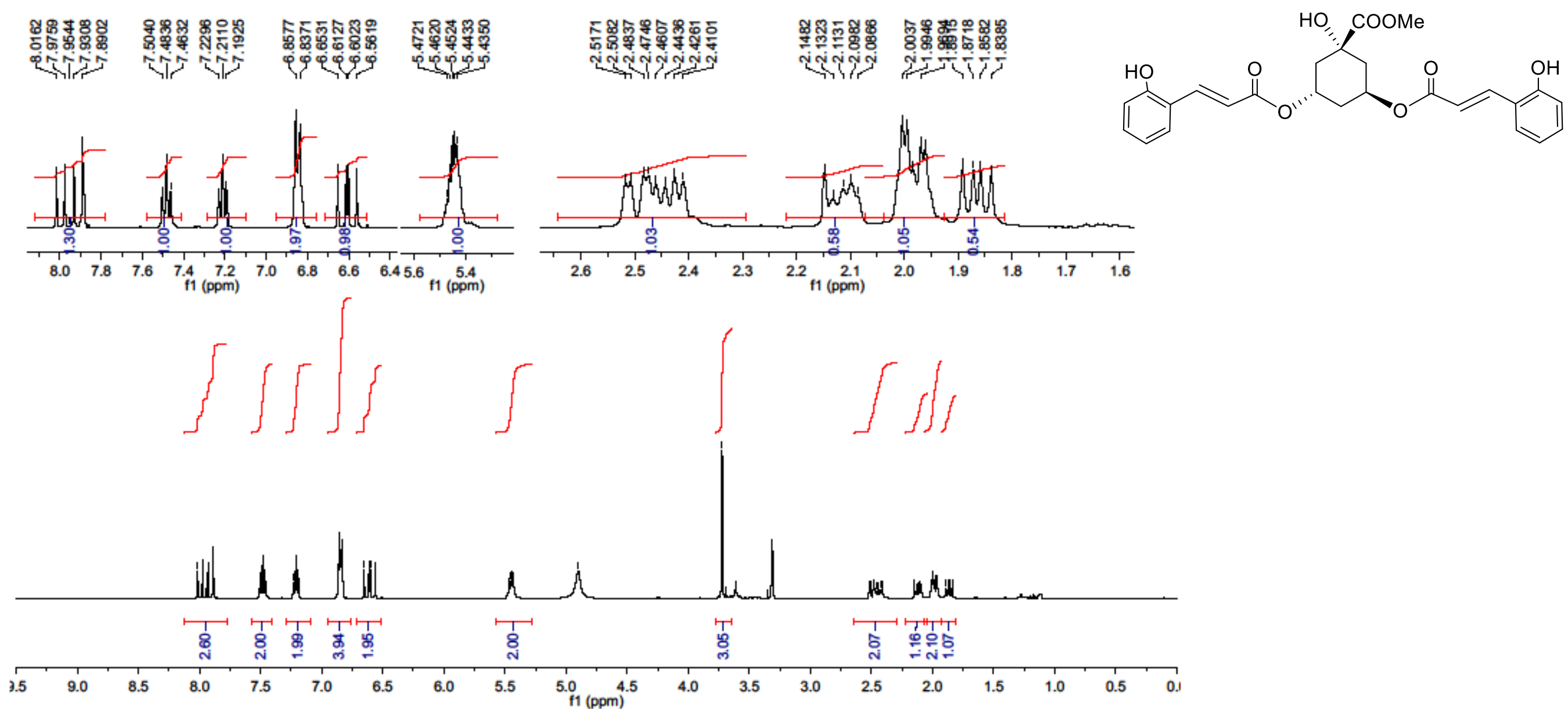
${ }^{13} \mathrm{C}$ NMR (100MHz, CD $\left.{ }_{3} \mathrm{OD}, 295 \mathrm{~K}\right)$
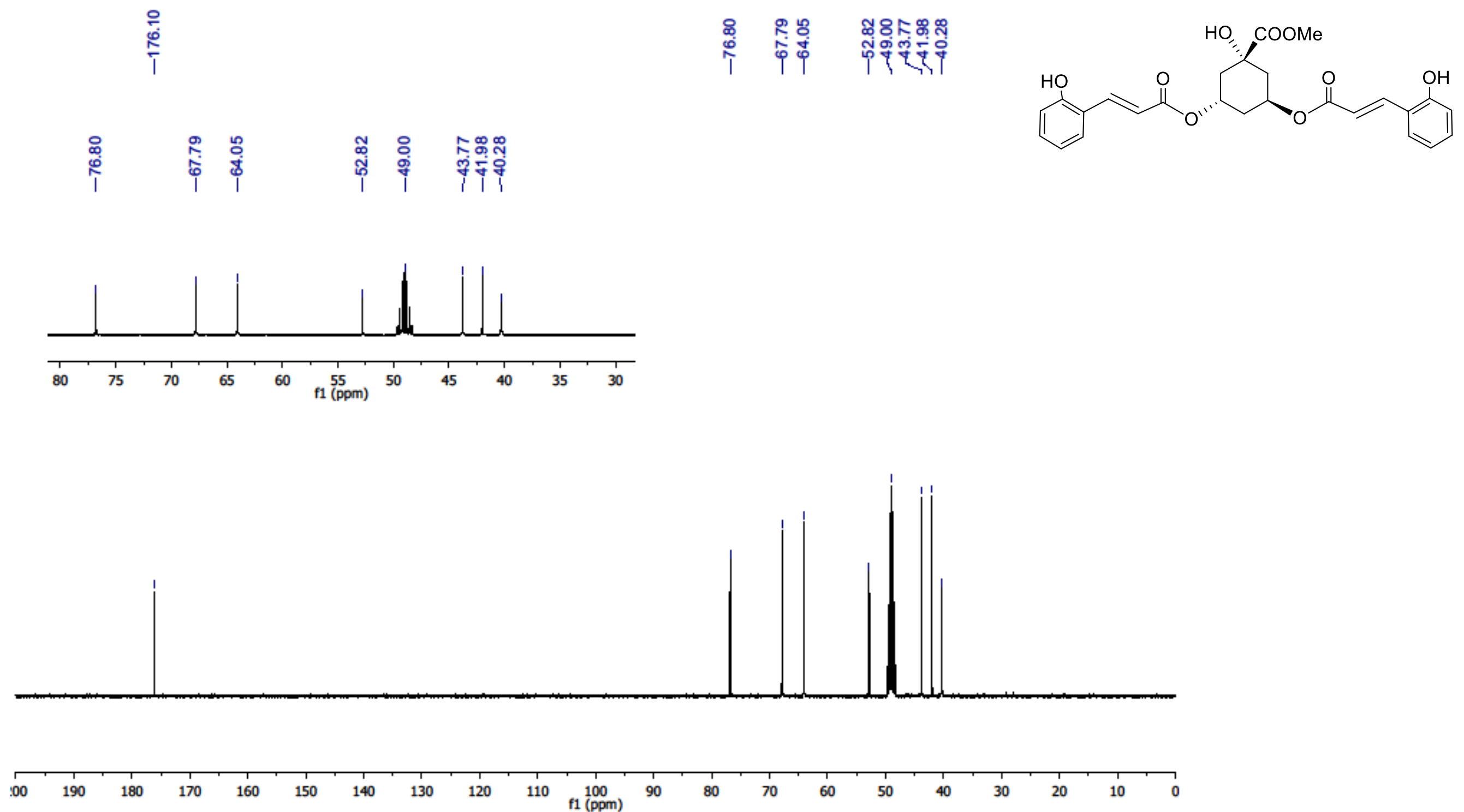
(3S,5S) 1-hydroxy-3,5 bis[[(2E)-3-(3,4-diacetoxyphenyl)-1-oxo-2-propen-1-yl]oxy]cyclohexanecarboxylic acid 11a

${ }^{1} \mathrm{H}$ NMR (400MHz, $\left.\mathrm{CD}_{3} \mathrm{OD}, 298 \mathrm{~K}\right)$

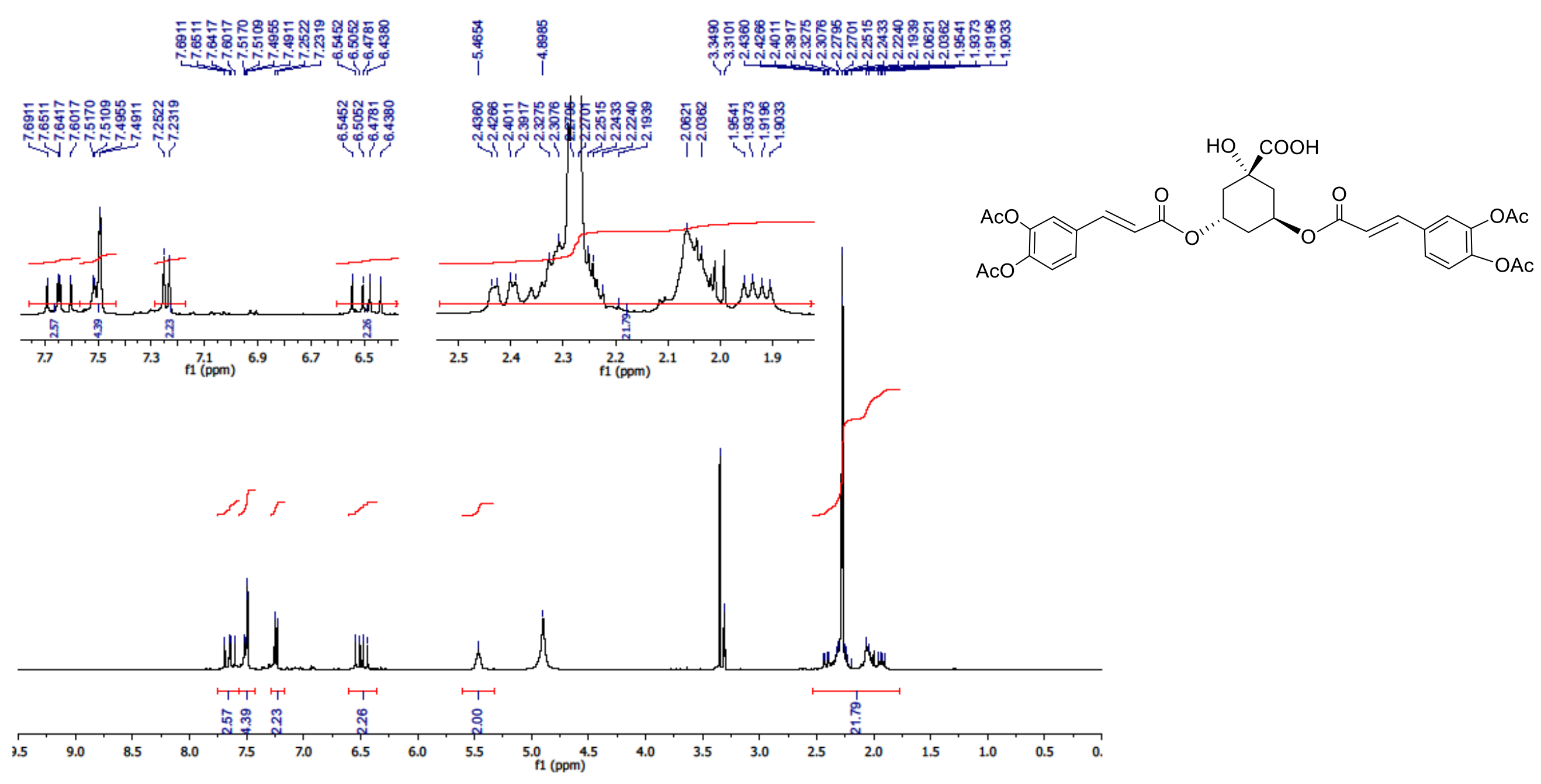


${ }^{13} \mathrm{C}$ NMR (100MHz, $\left.\mathrm{CD}_{3} \mathrm{OD}, 298 \mathrm{~K}\right)$
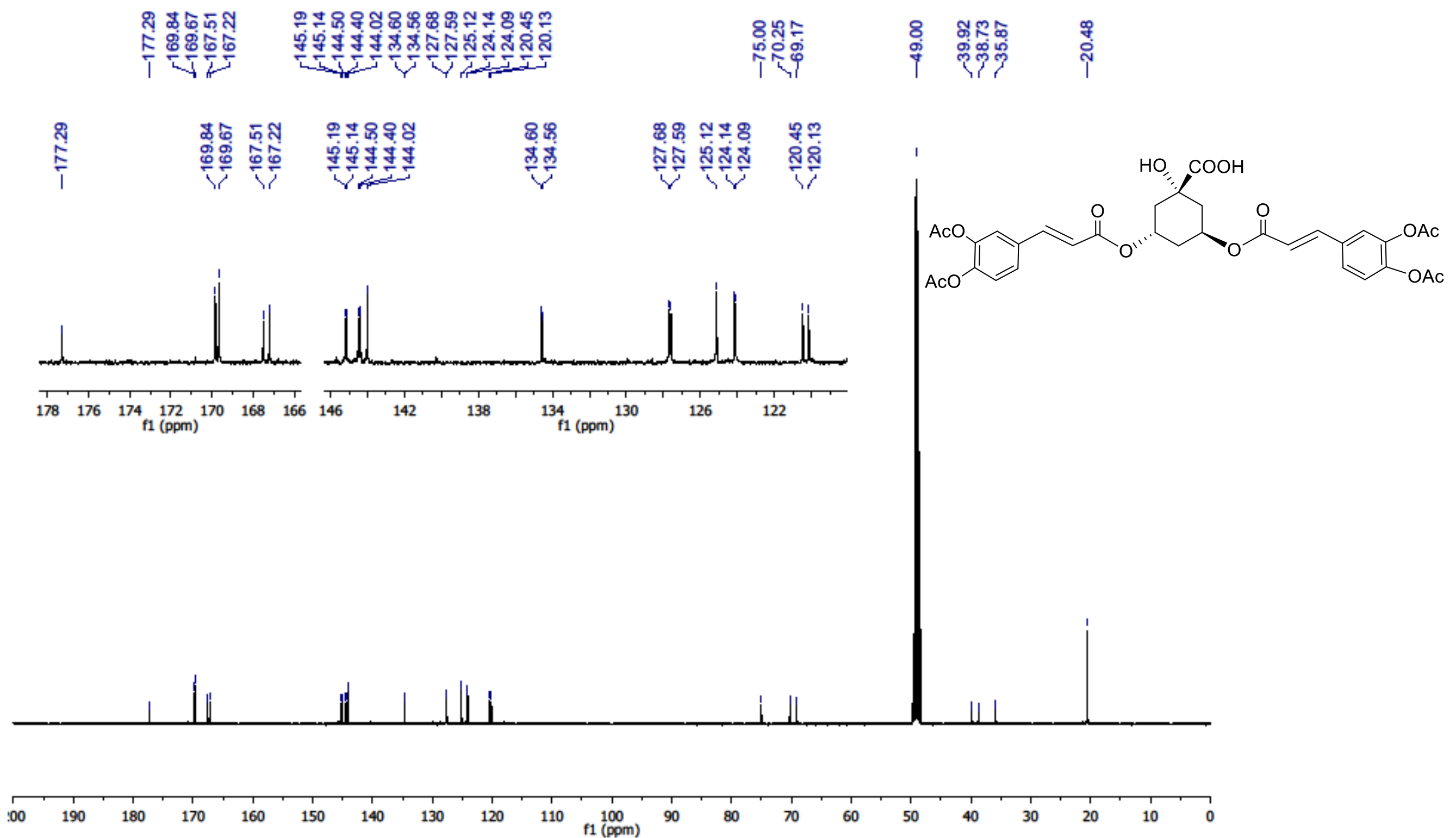
(3S,5S) 1-hydroxy-3,5 bis[[(2E)-3-(4-acetoxy-3-methoxyphenyl)-1-oxo-2-propen-1-yl]oxy]cyclohexanecarboxylic acid 11c

${ }^{1}$ H NMR (400MHz, $\left.\mathrm{CDCl}_{3}, 295 \mathrm{~K}\right)$

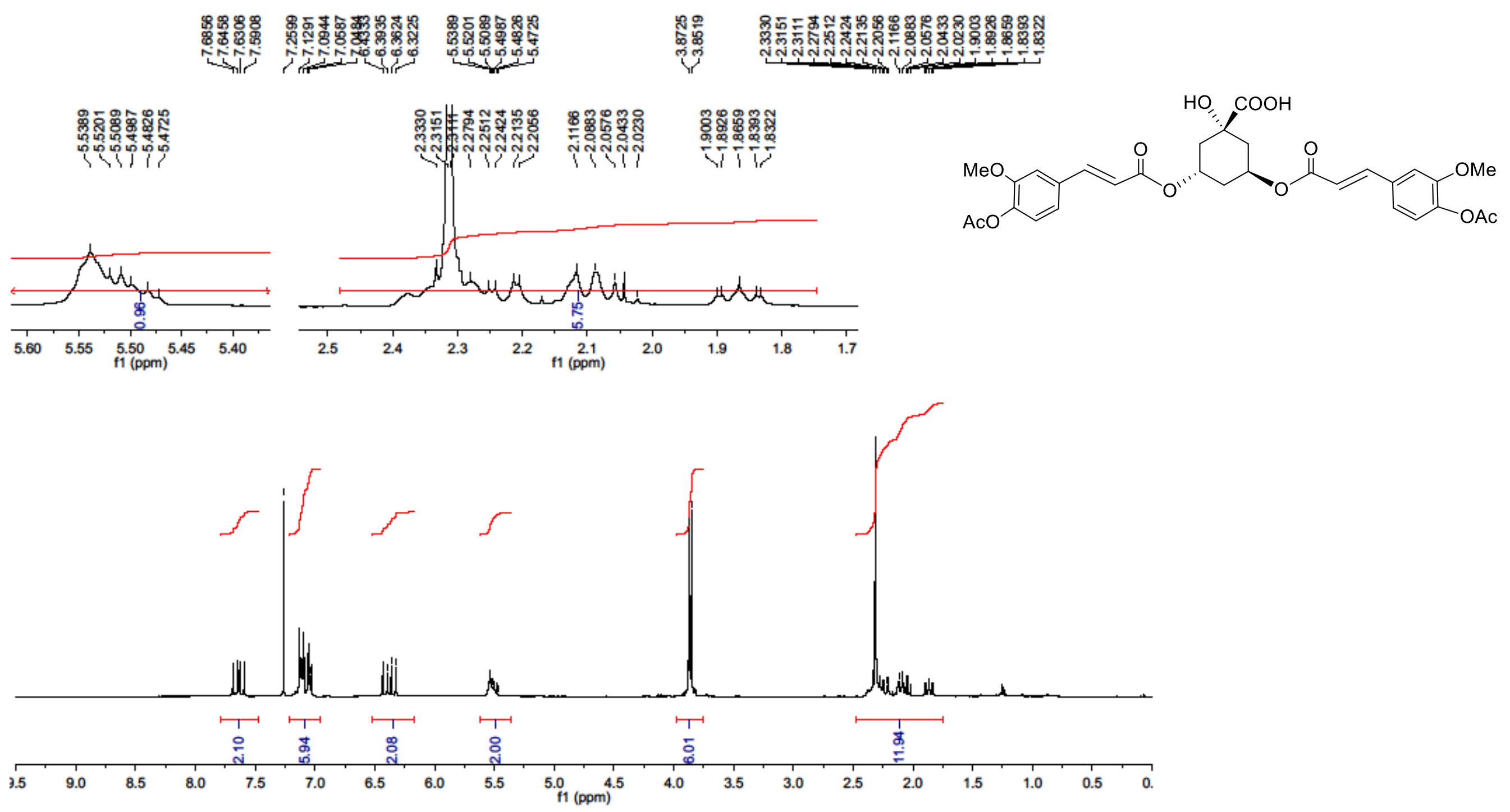


${ }^{13} \mathrm{C}$ NMR (100MHz, CD $\left.{ }_{3} \mathrm{OD}, 295 \mathrm{~K}\right)$

\begin{tabular}{|c|c|c|c|c|c|}
\hline 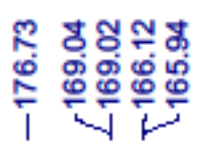 & 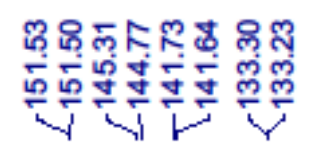 & 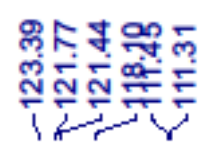 & $\begin{array}{l}\text { 유웡 } \\
\text { F尔安 }\end{array}$ & 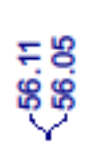 & 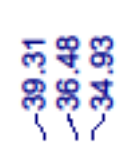 \\
\hline
\end{tabular}
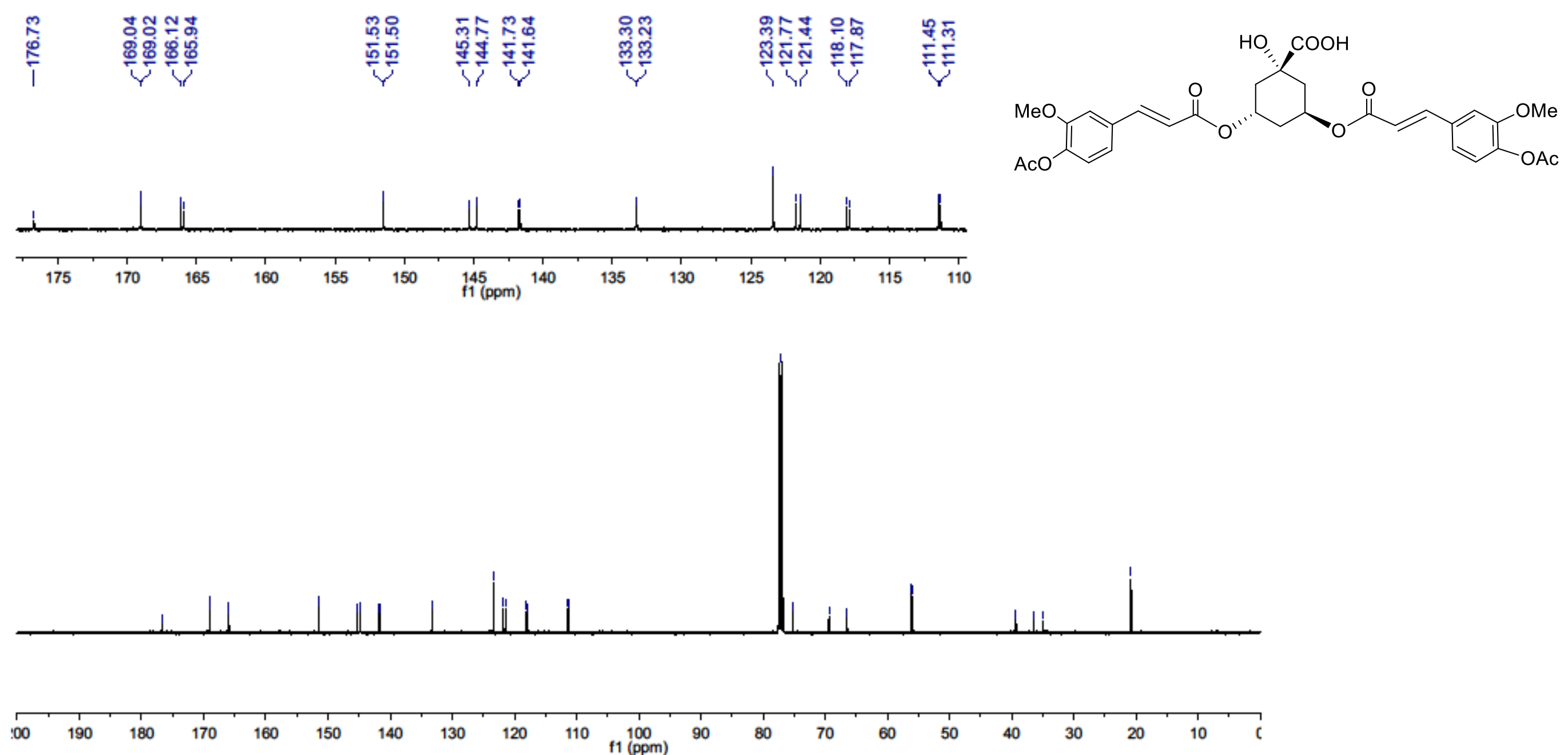
(3S,5S) 1-hydroxy-3,5 bis[[(2E)-3-(4-hydroxyphenyl)-1-oxo-2-propen-1-yl]oxy]cyclohexanecarboxylic acid 11d

${ }^{1}$ H NMR (400MHz, $\left.\mathrm{CDCl}_{3}, 295 \mathrm{~K}\right)$

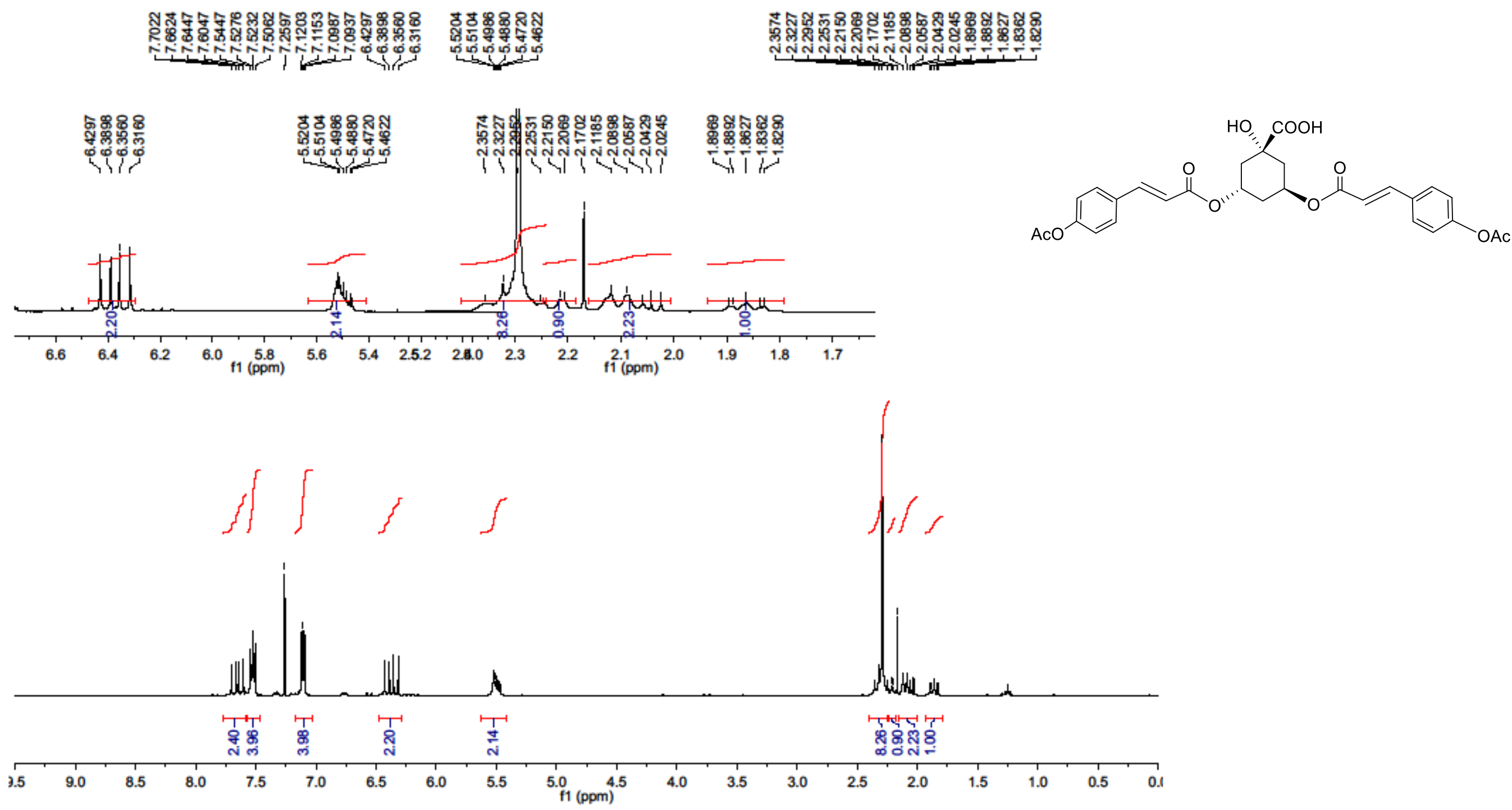


${ }^{13} \mathrm{C}$ NMR (100MHz, CD $\left.{ }_{3} \mathrm{OD}, 295 \mathrm{~K}\right)$
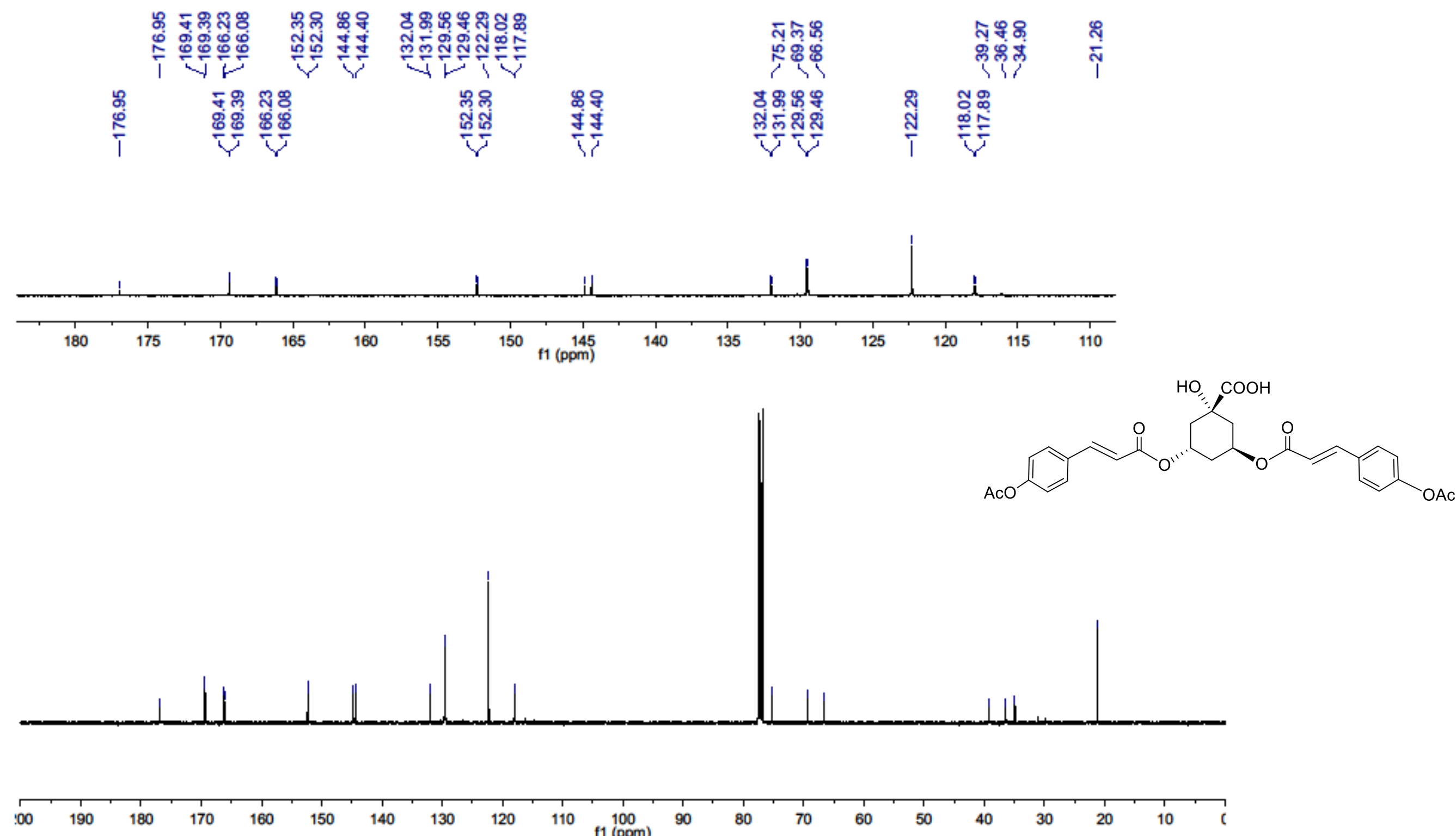
(3S,5S) 1-hydroxy-3,5 bis[[(2E)-3-(3,4-dihydroxyphenyl)-1-oxo-2-propen-1-yl]oxy]cyclohexanecarboxylic acid 4a

${ }^{1} \mathrm{H}$ NMR (400MHz, $\left.\mathrm{CDCl}_{3}, 295 \mathrm{~K}\right)$

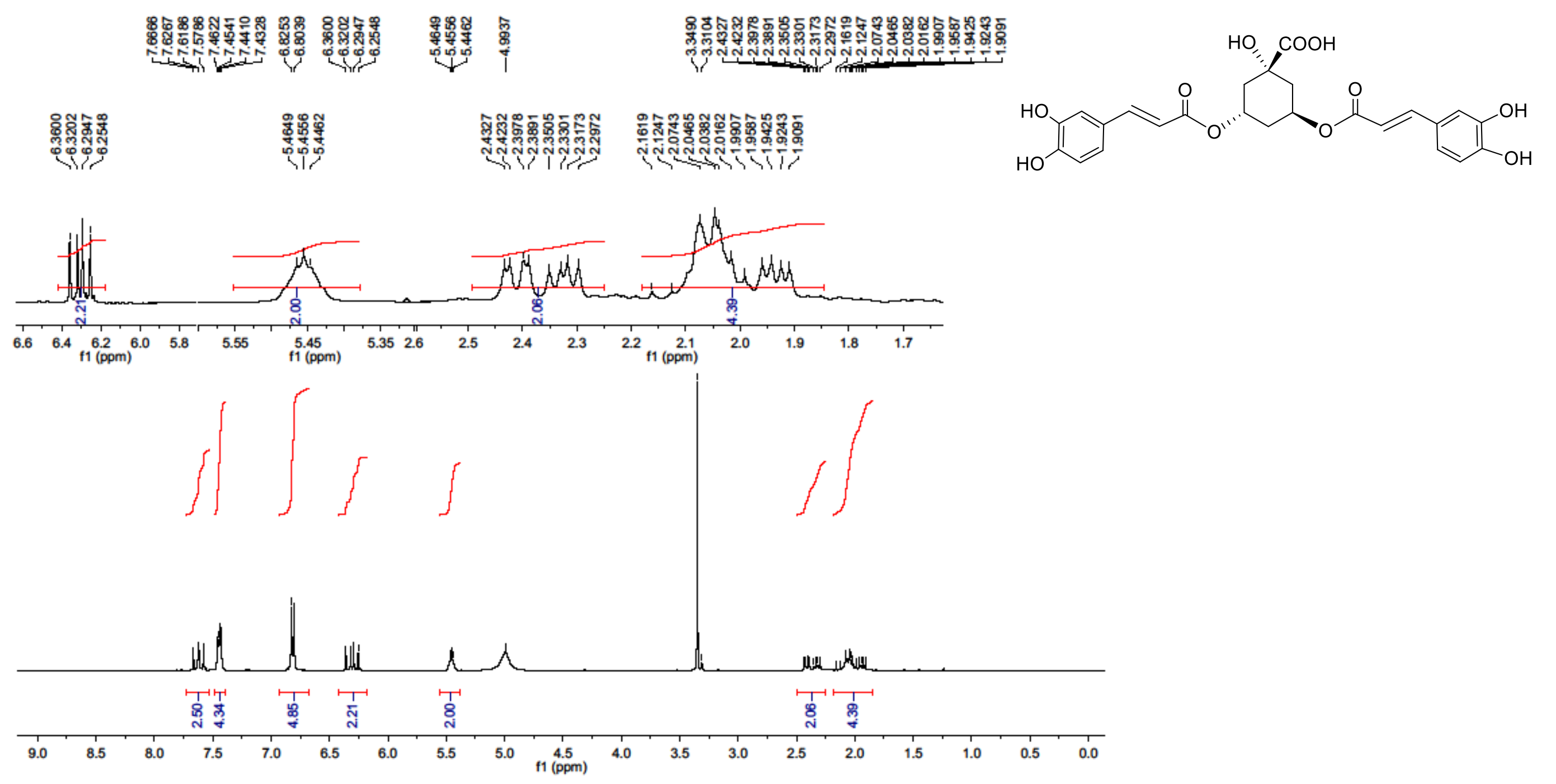


${ }^{13} \mathrm{C}$ NMR (100MHz, $\left.\mathrm{CD}_{3} \mathrm{OD}, 295 \mathrm{~K}\right)$

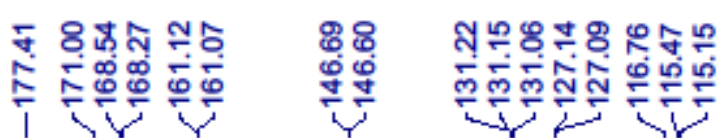

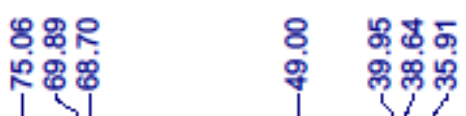

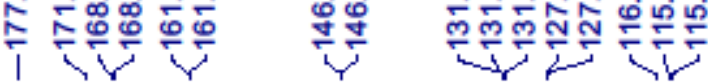

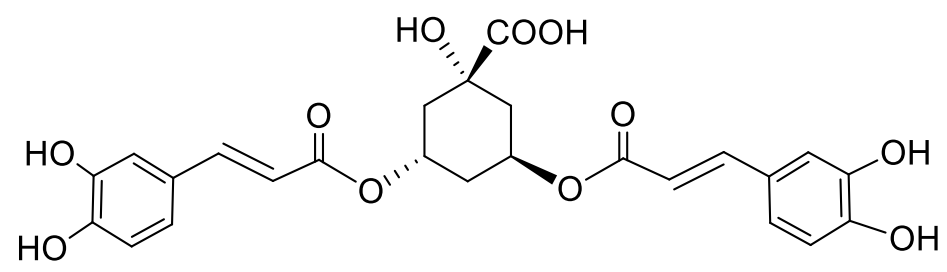

๘

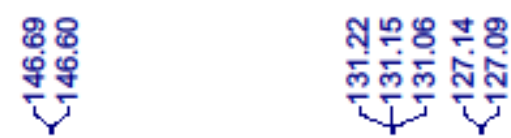

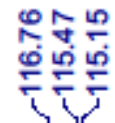
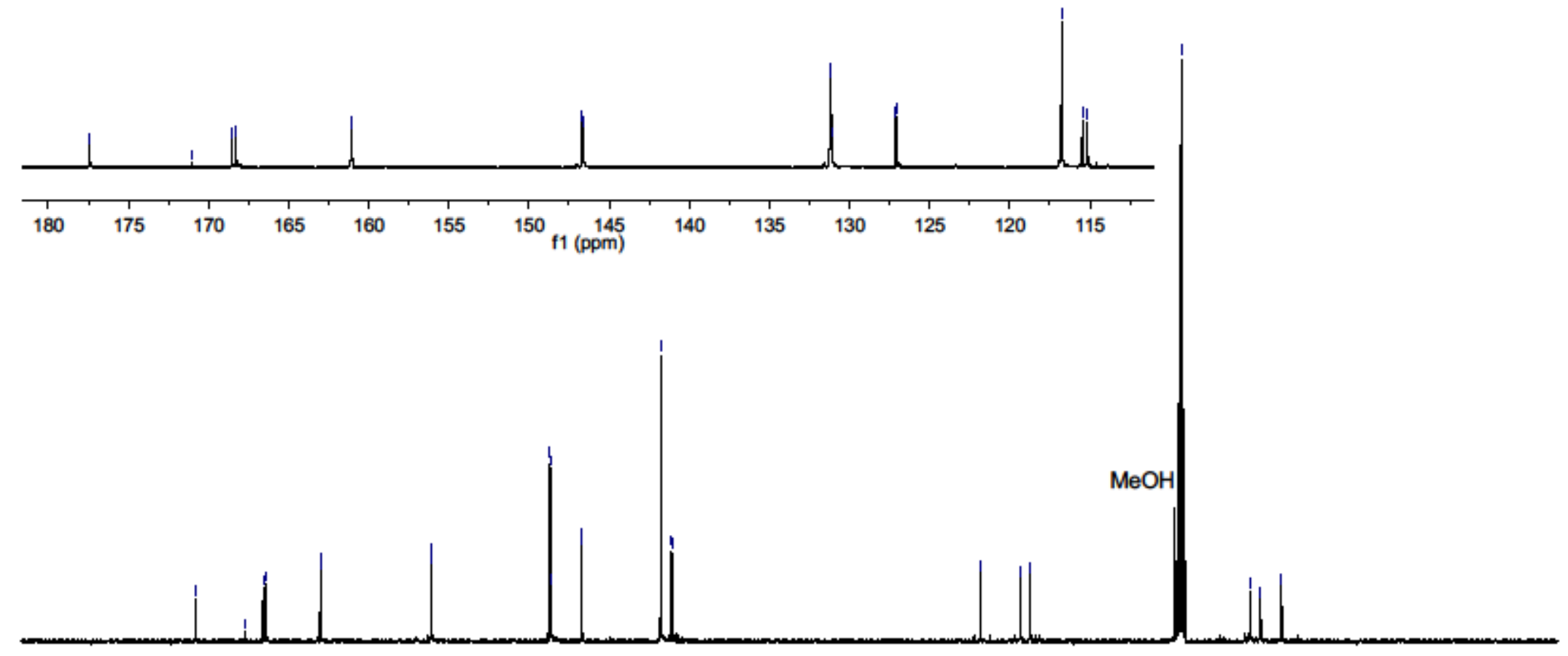

$: 00$

$190 \quad 180$

160

$140 \quad 130$

120

$110 \quad 100$
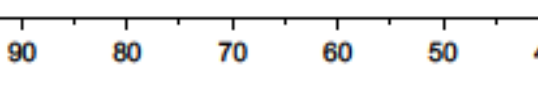

40

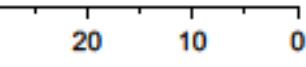


(3S,5S) 1-hydroxy-3,5 bis[[(2E)-3-(4-hydroxy-3-methoxyphenyl)-1-oxo-2-propen-1-yl]oxy]cyclohexanecarboxylic acid 4c

${ }^{1} \mathrm{H}$ NMR (400MHz, $\left.\mathrm{CD}_{3} \mathrm{OD}, 297 \mathrm{~K}\right)$
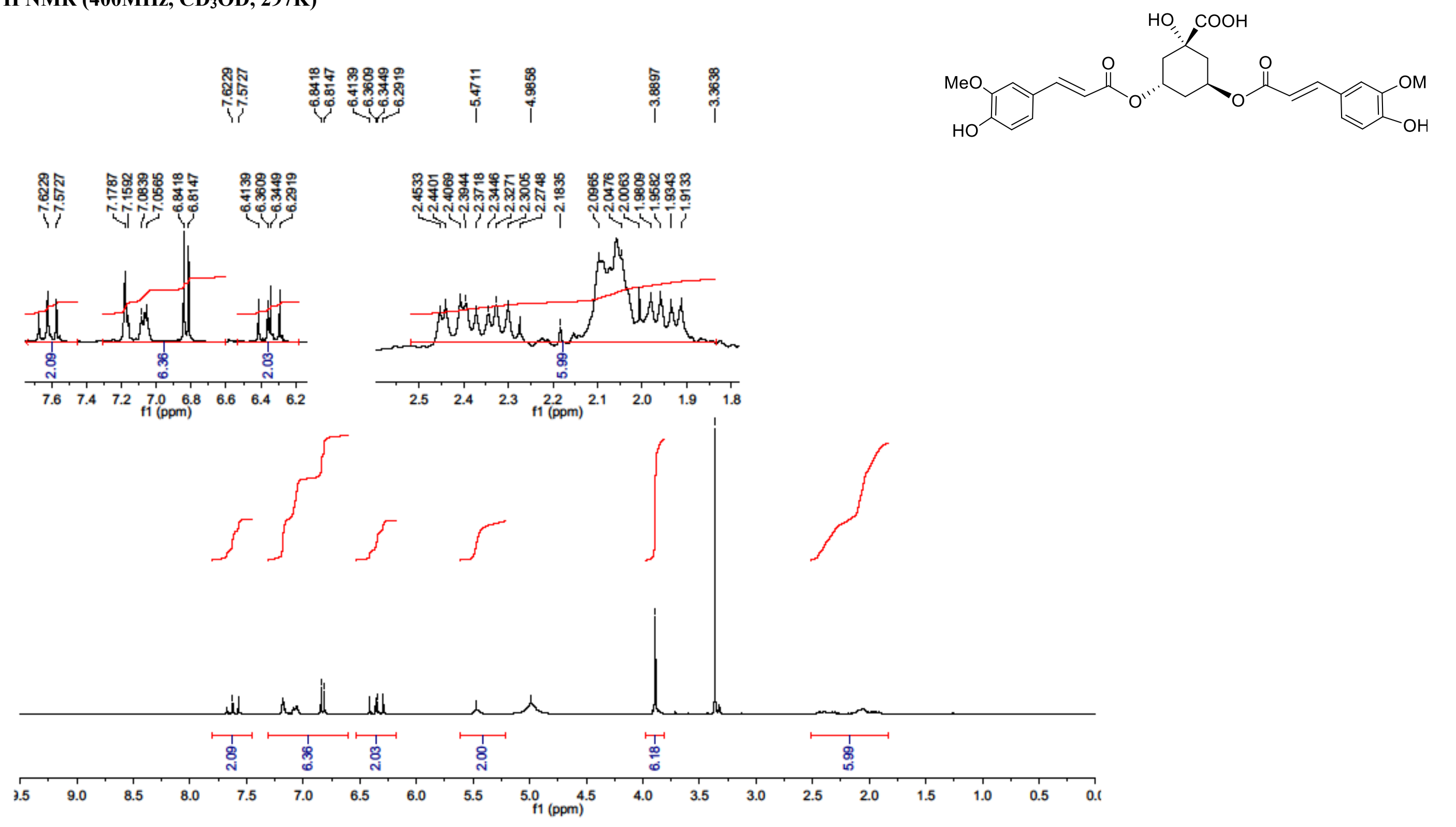
${ }^{13} \mathrm{C}$ NMR (100MHz, CD $\left.{ }_{3} \mathrm{OD}, 297 \mathrm{~K}\right)$
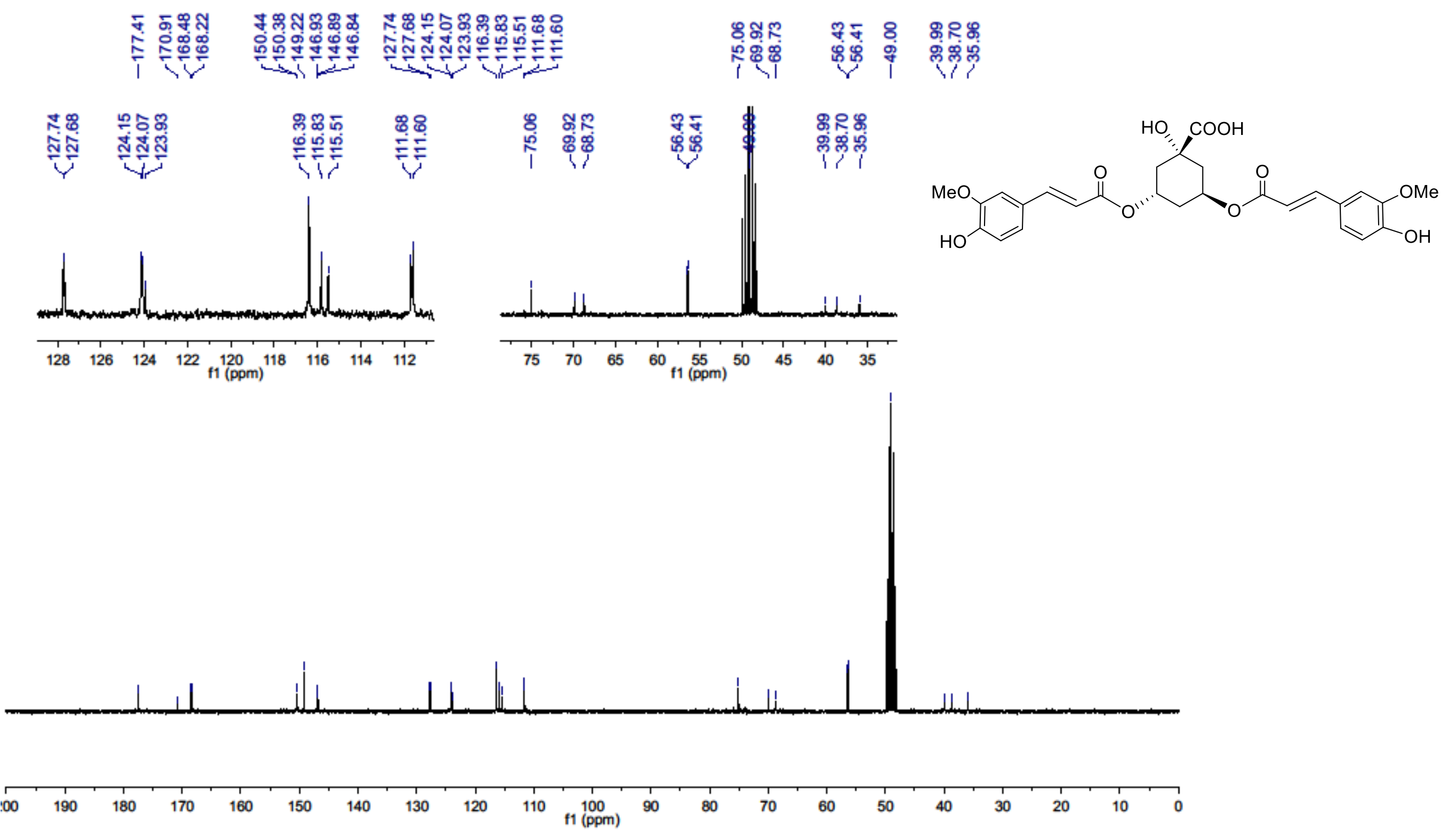
(3S,5S) 1-hydroxy-3,5 bis[[(2E)-3-(4-hydroxyphenyl)-1-oxo-2-propen-1-yl]oxy]cyclohexanecarboxylic acid 4d

${ }^{1}$ H NMR (400MHz, $\left.\mathrm{CDCl}_{3}, 295 \mathrm{~K}\right)$
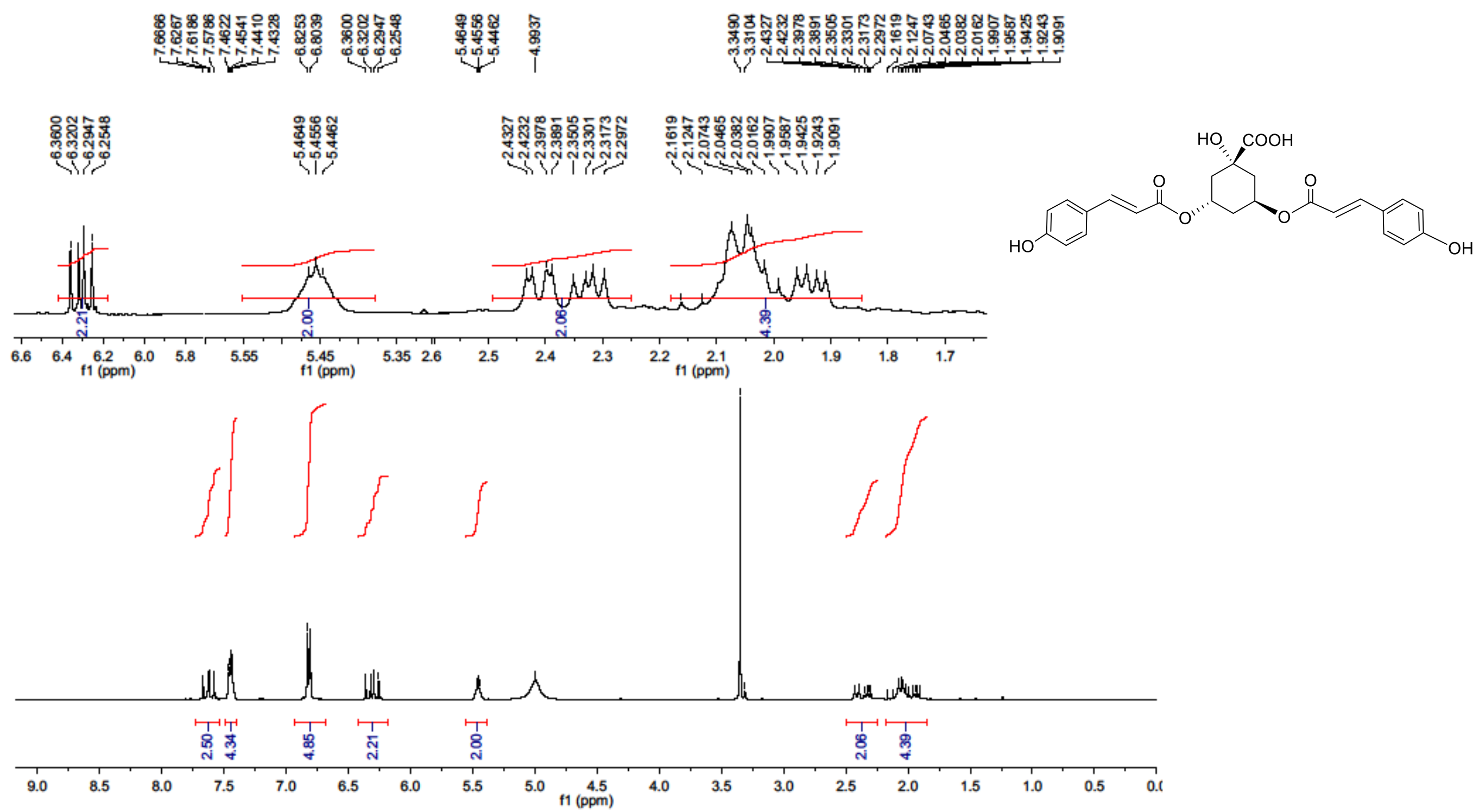
${ }^{13} \mathrm{C}$ NMR (100MHz, $\left.\mathrm{CD}_{3} \mathrm{OD}, 295 \mathrm{~K}\right)$

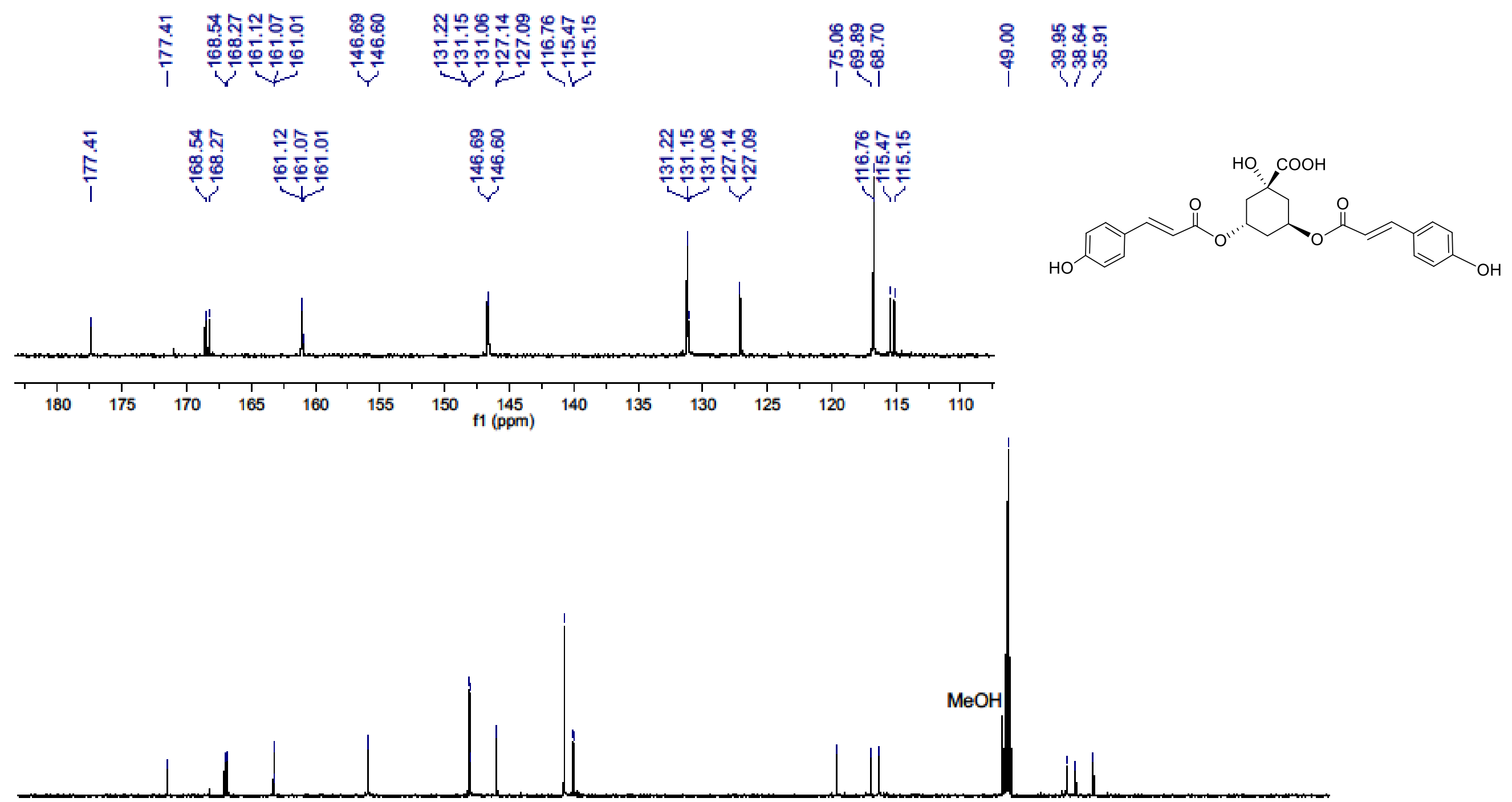

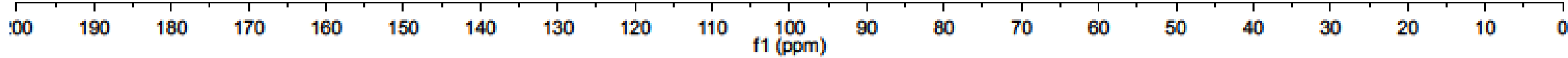


(3R, 3aR, 6S, 6aR)-hexahydrofuro[3,2-b]furan-3,6-diyl (2E,2'E)bis--[4-(acetyloxy)-3-methoxyphenyl]prop-2-enoate I1

NMR ${ }^{1} \mathrm{H}\left(400 \mathrm{MHz}, \mathrm{CDCl}_{3}, 295 \mathrm{~K}\right)$

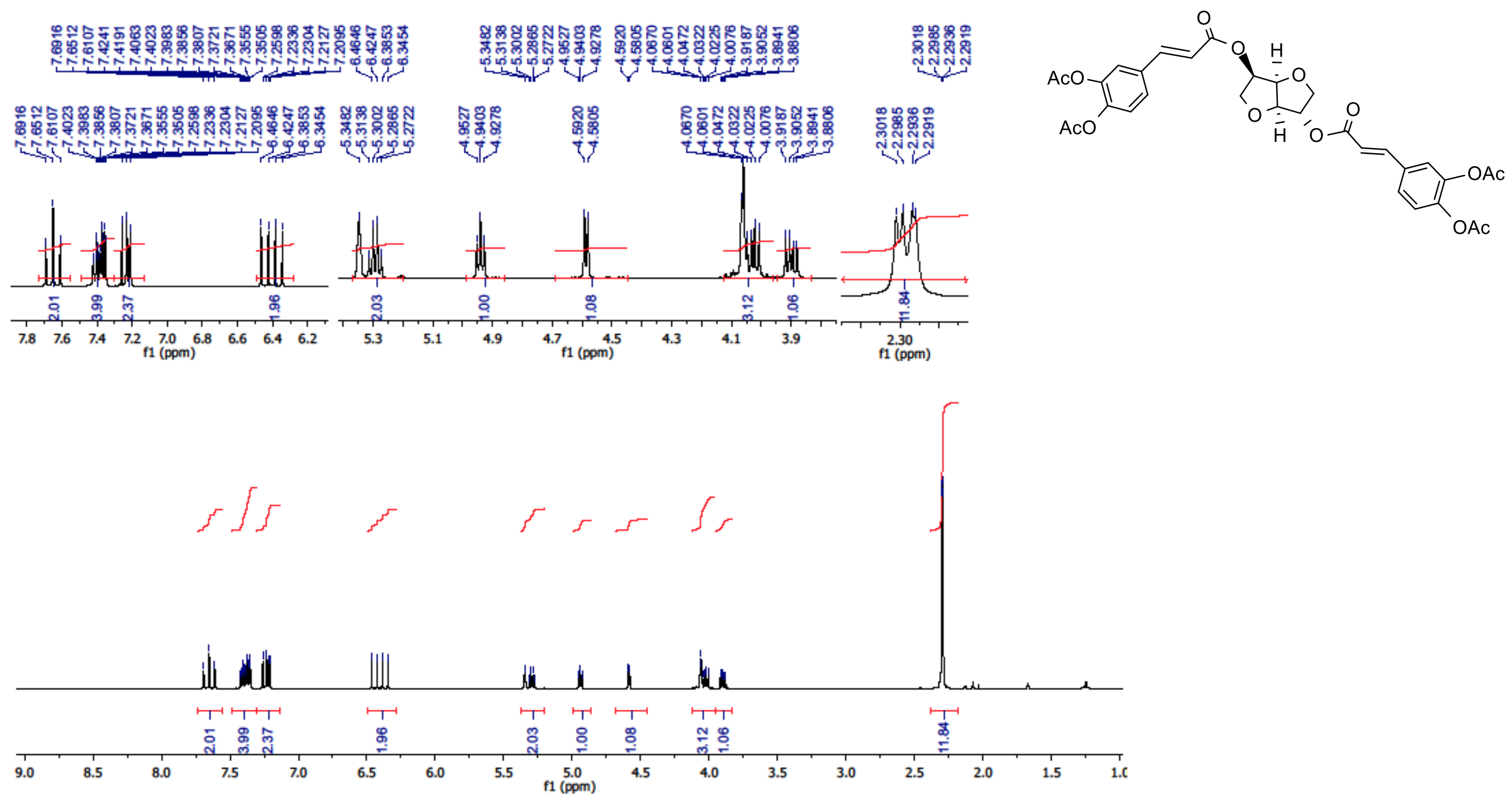


NMR ${ }^{13} \mathrm{C}\left(100 \mathrm{MHz}, \mathrm{CDCl}_{3}\right)$

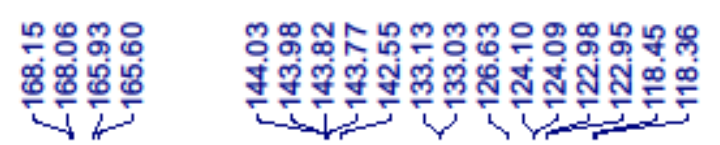

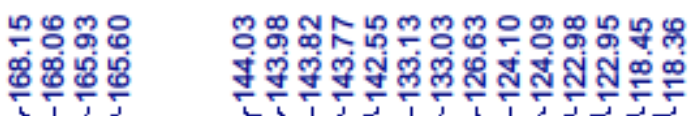

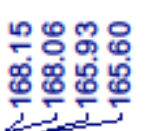

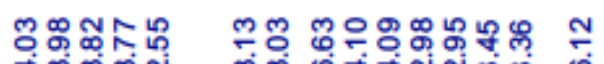

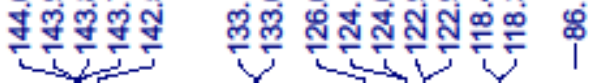
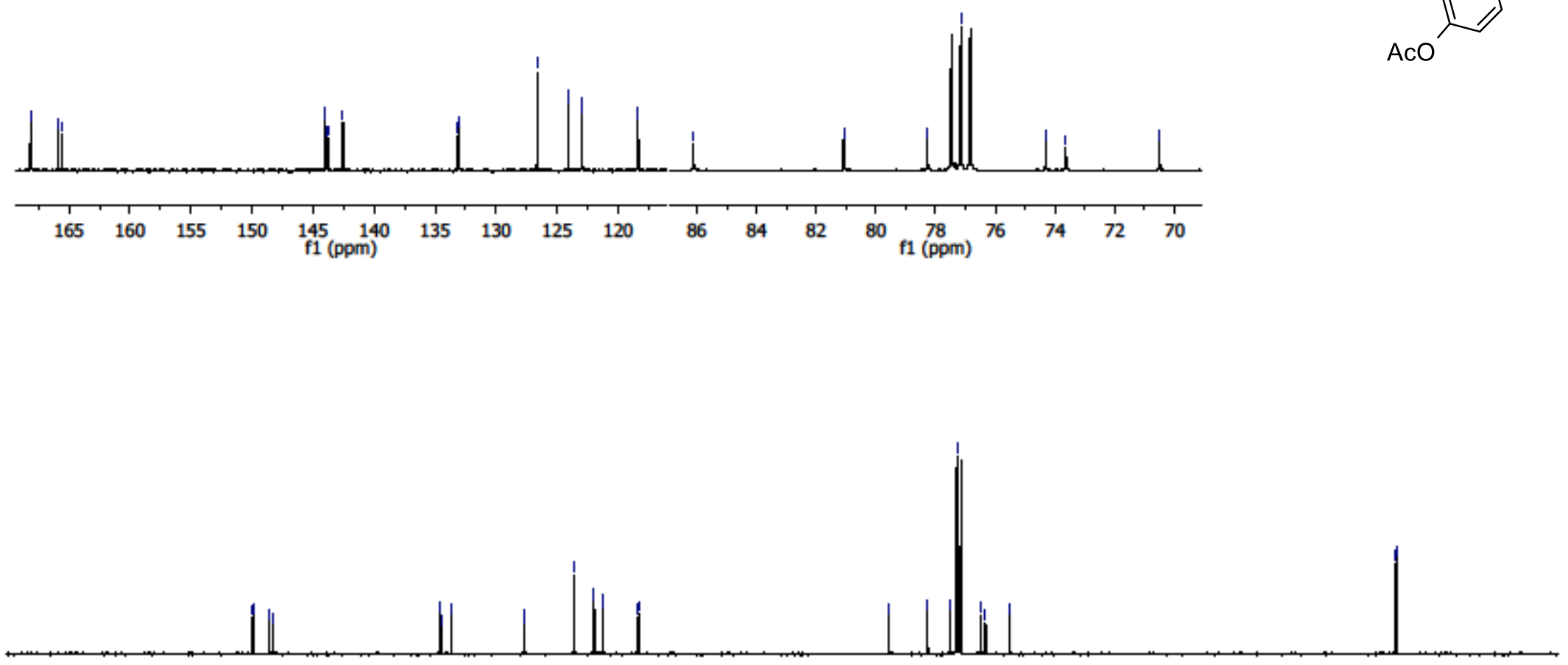

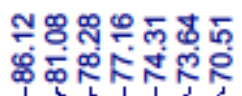

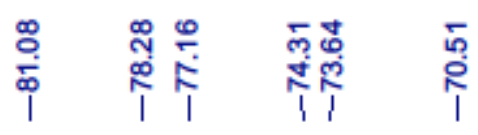

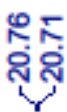

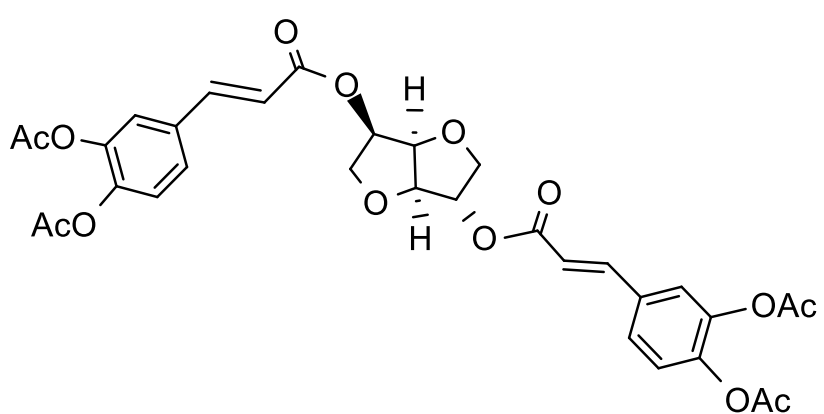

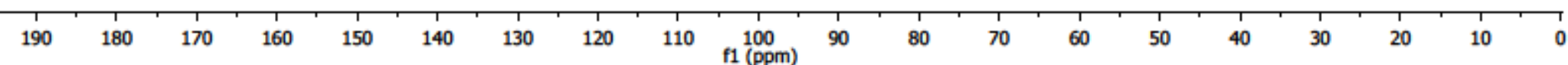


$(3 R, 3 a R, 6 S, 6 a R)$-hexahydrofuro[3,2-b]furan-3-yl (2E)-3-(3,4-dihydroxyphenyl)prop-2-enoate 12 NMR ${ }^{1} \mathrm{H}\left(300 \mathrm{MHz}, \mathrm{CD}_{3} \mathrm{OD}\right)$
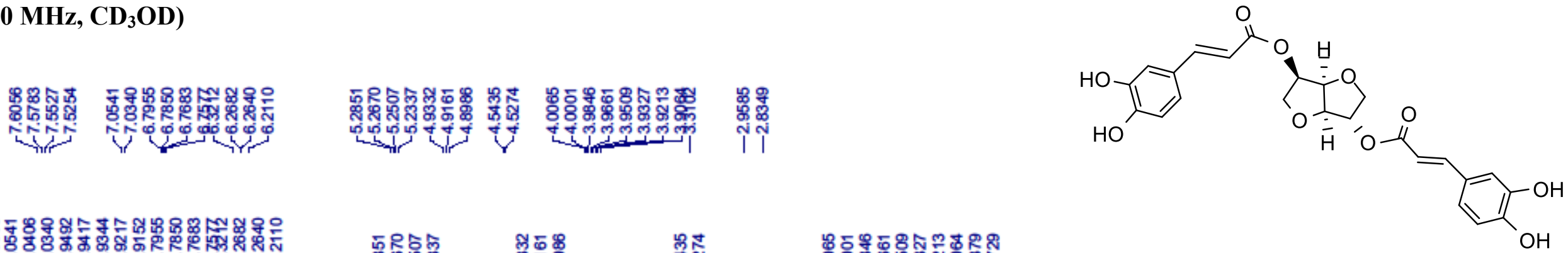

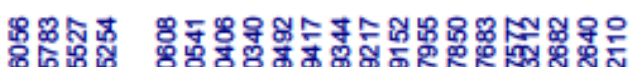

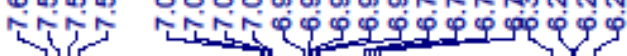
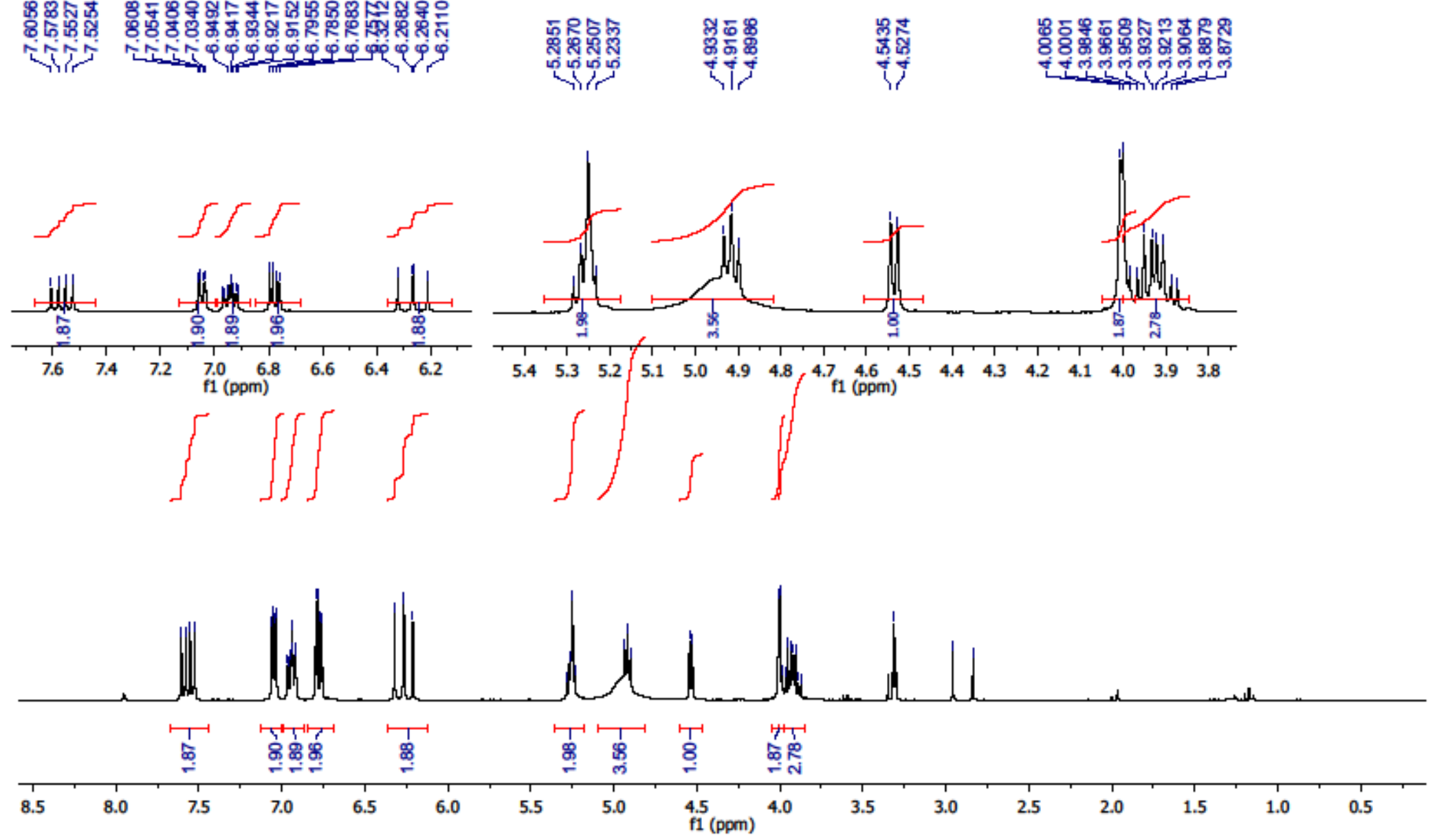
NMR ${ }^{13} \mathrm{C}$ (75 MHz, CD 0 OD)
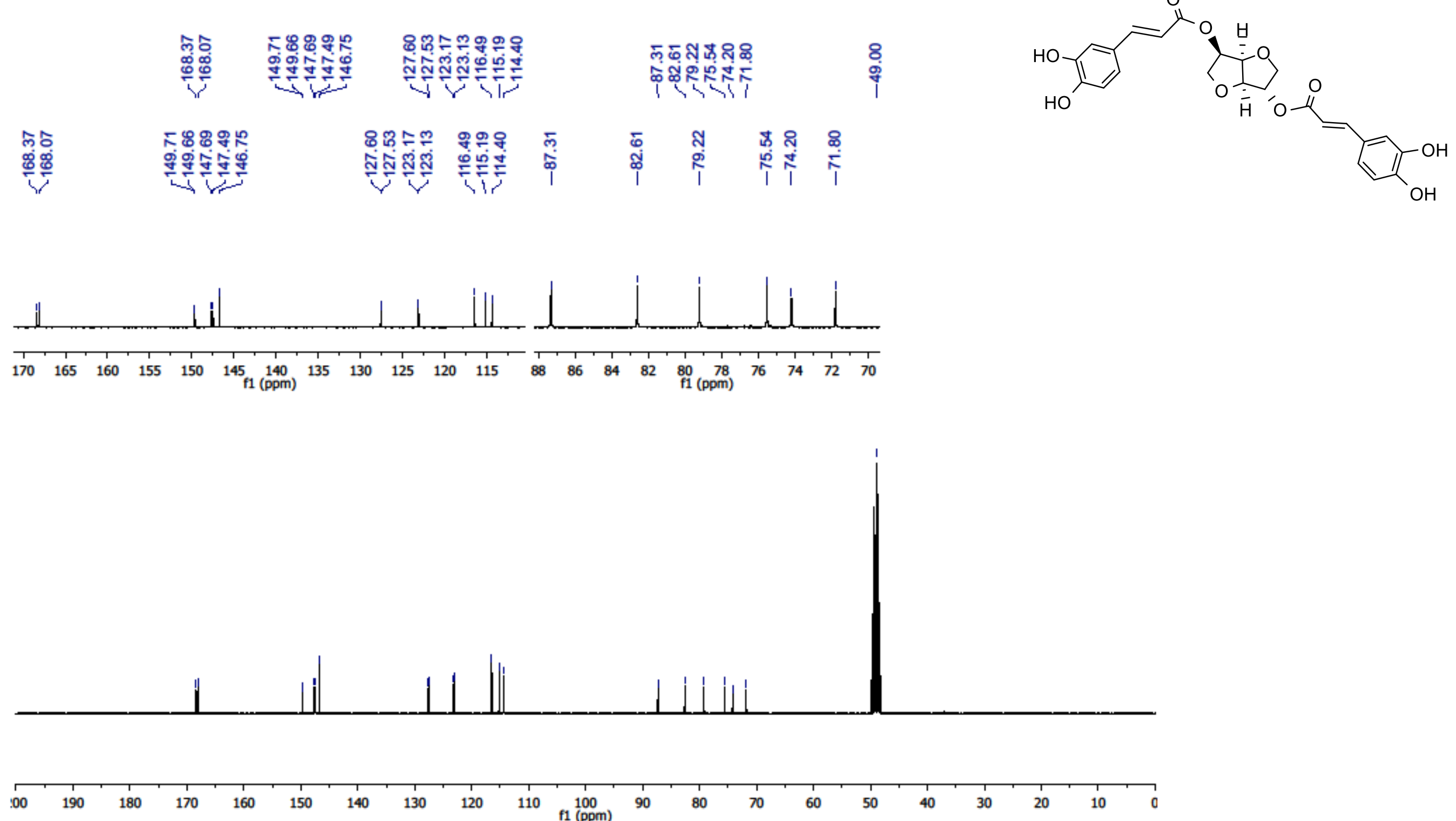
${ }^{1} \mathrm{H}$ NMR Spectra of $4 \mathrm{a}$ and $4 \mathrm{~d}$ in $\mathrm{CD}_{3} \mathrm{OD}$ at $296,5 \mathrm{~K}$ at different time intervals $\left(\mathrm{t}_{0}+6 \mathrm{~h}, \mathrm{t}_{0}+24 \mathrm{~h}, \mathrm{t}_{0}+48 \mathrm{~h}, \mathrm{t}_{0}+60 \mathrm{~h}\right)$
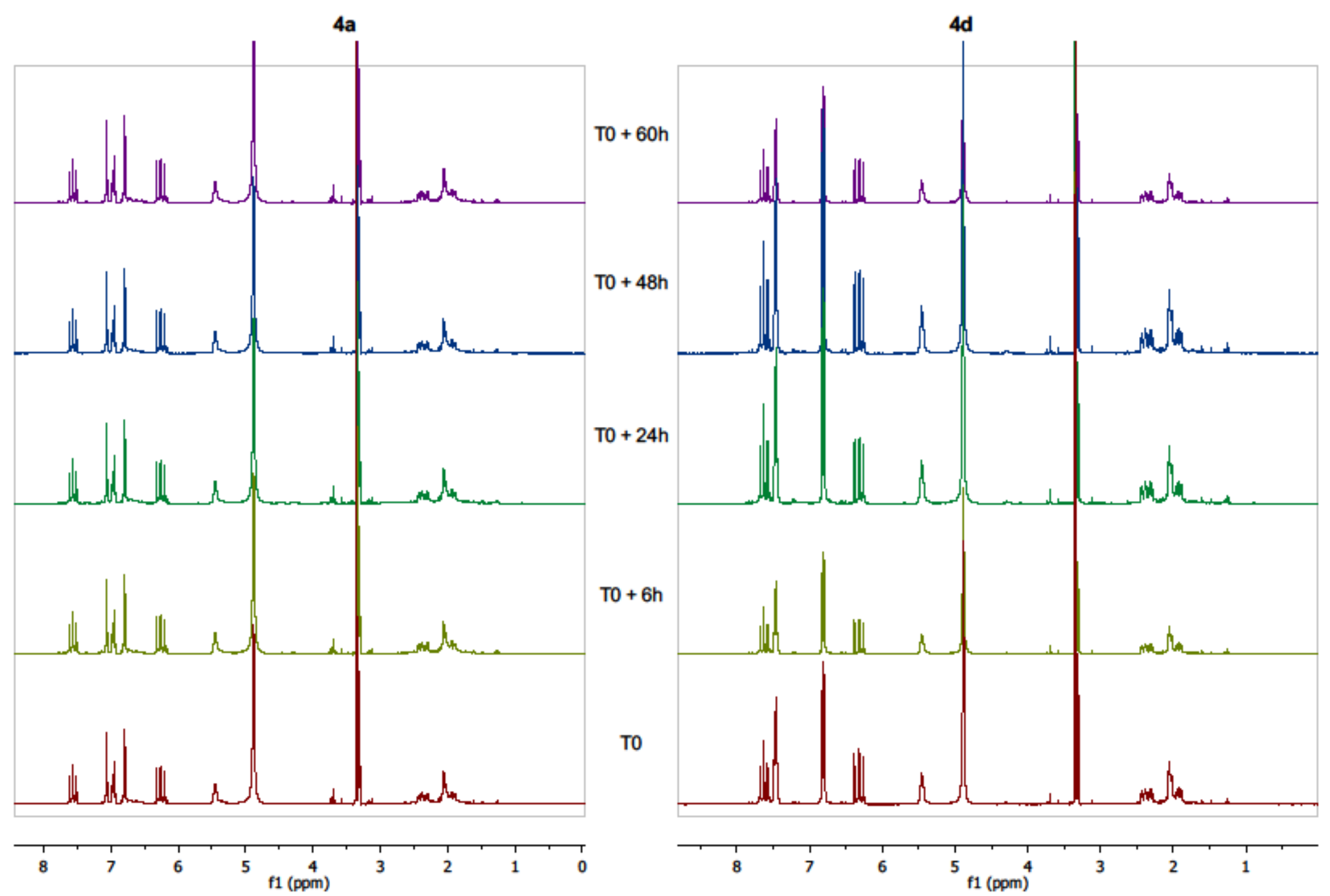
HPLC conditions : Agilent equipment, Column phenomenex C18: Jupiter ${ }^{\circledR} 5 \mu \mathrm{m} ; 300 \AA$, LC Column $250 \mathrm{x}$ 4.6mm, flow 1ml/min; Mobile phase 0 - 2min: 80\% $\mathrm{H}_{2} \mathrm{O}$ UHQ + TFA (A) / 20\% $\mathrm{CH}_{3} \mathrm{CN}+\mathrm{TFA}(\mathrm{B})$; 22min: $40 \%$ A / 60\% B; 30min: 100\% ACN + TFA; 33min: 80\% A/ 20\% B. Samples were dissolved in $\mathrm{MeOH}(6 \mathrm{~mL})$ and $10 \mu \mathrm{g}$ were injected per sample

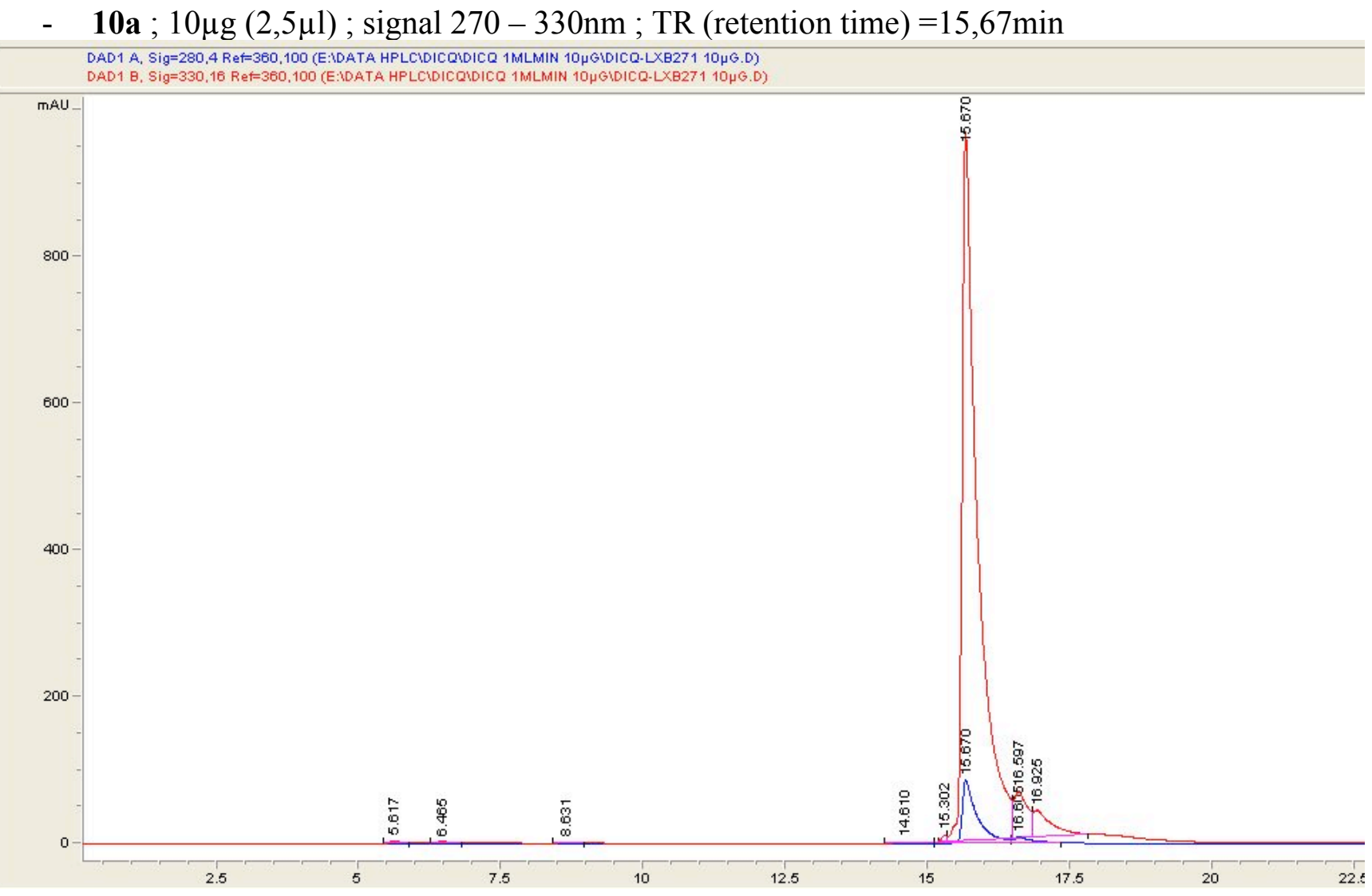

UV spectrum $(210-400 \mathrm{~nm})$ à $15,670 \mathrm{~min}($ signal 330nm)

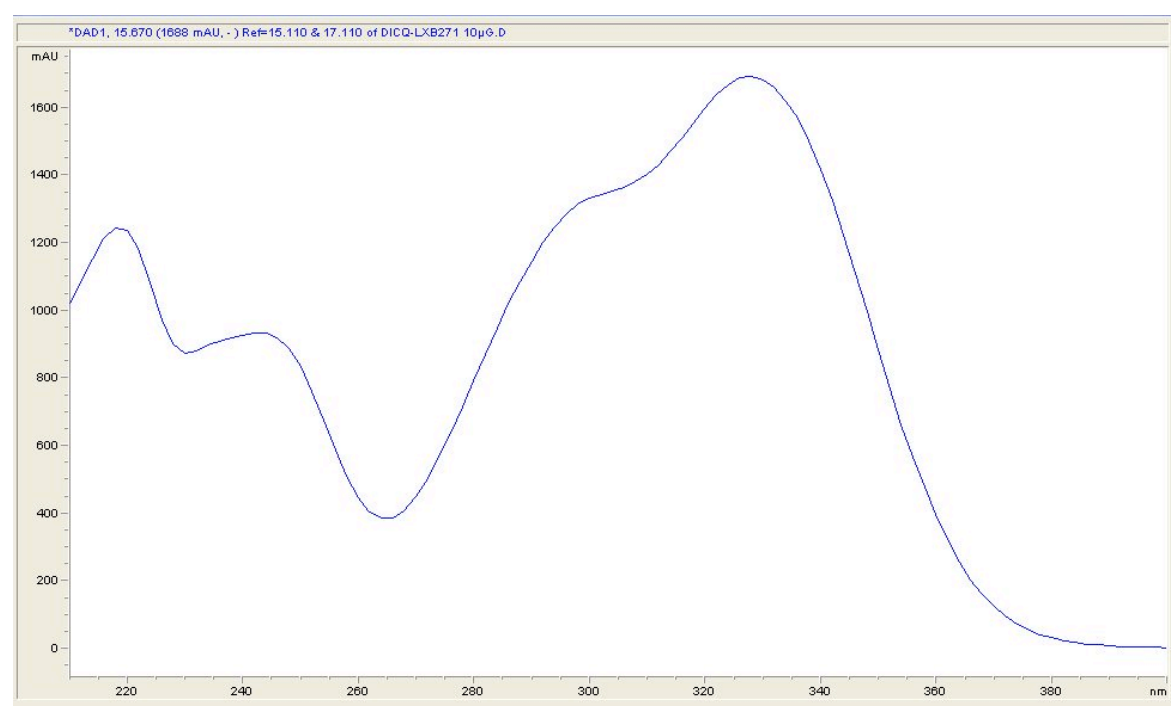


- 10c $10 \mu \mathrm{g}(4 \mu \mathrm{l}) ; 270-330 \mathrm{~nm} ; \mathrm{TR}=19,95 \mathrm{~min}$

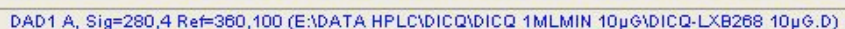

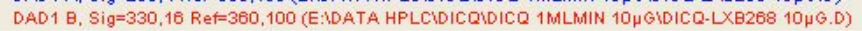

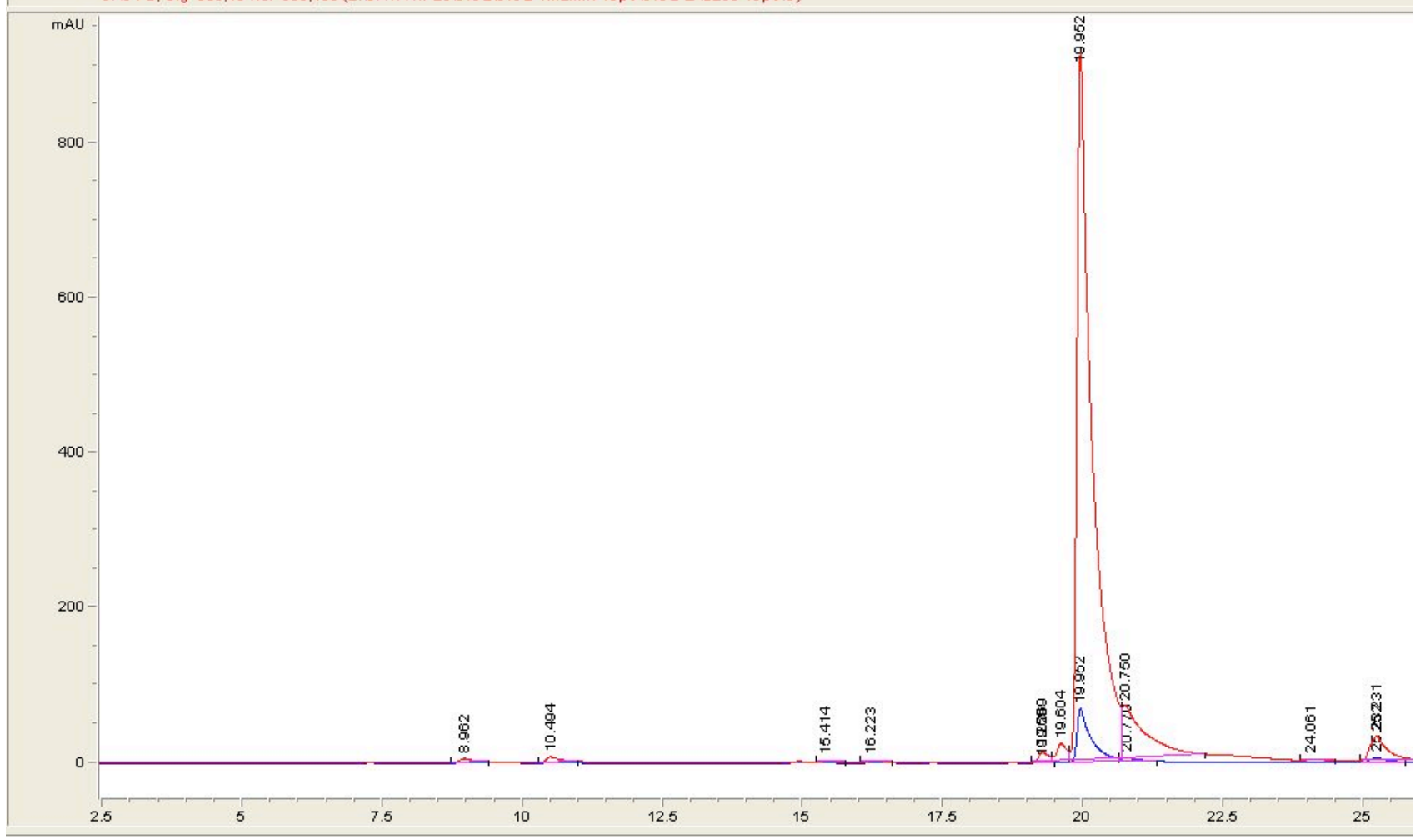

Spectre UV (210 - 400nm) à 19,893min (signal 330nm)

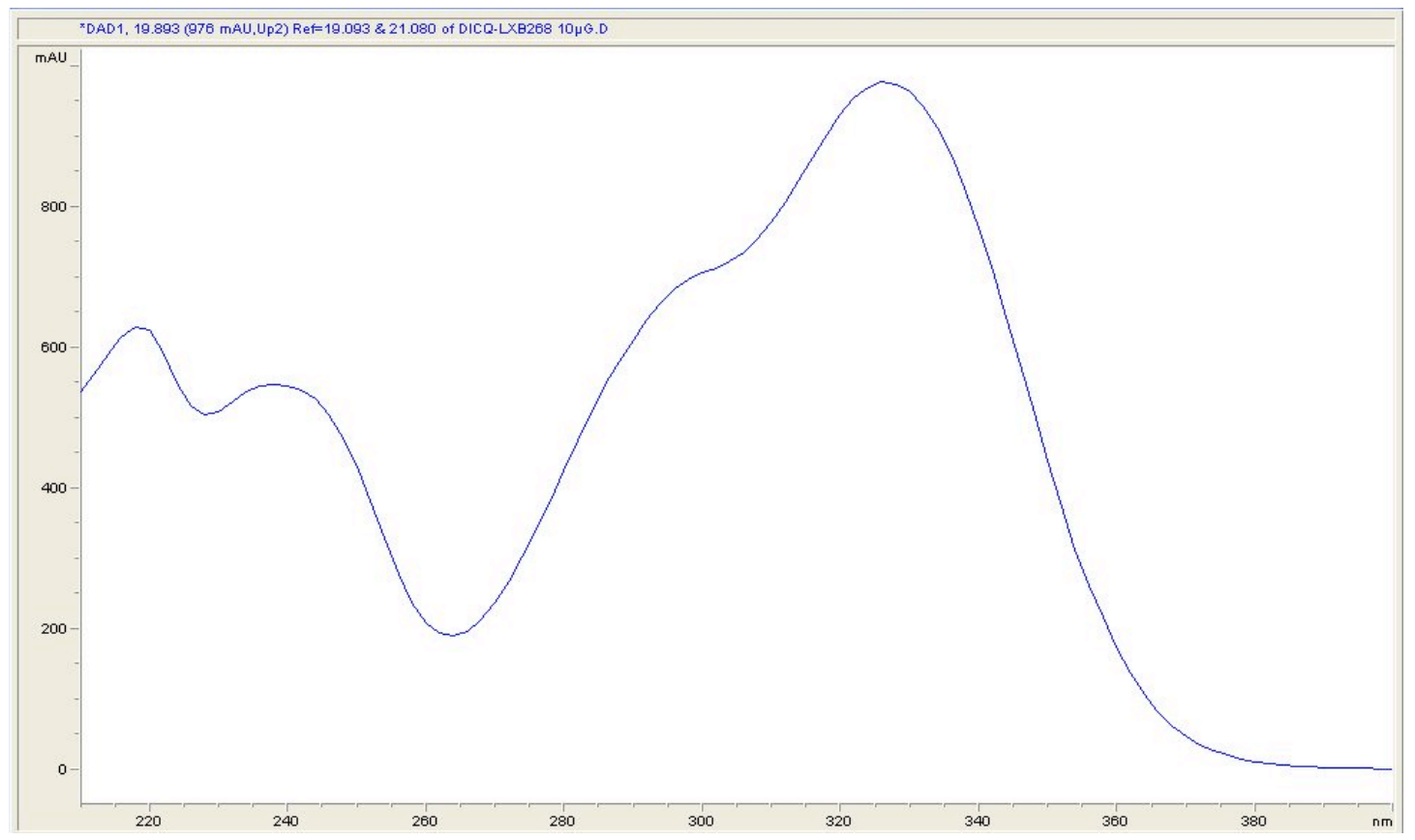


- $\quad$ 10d $; 10 \mu \mathrm{g}(8,5 \mu \mathrm{l}) ; 270-330 \mathrm{~nm} ; \mathrm{TR}=22,3 \mathrm{~min}$

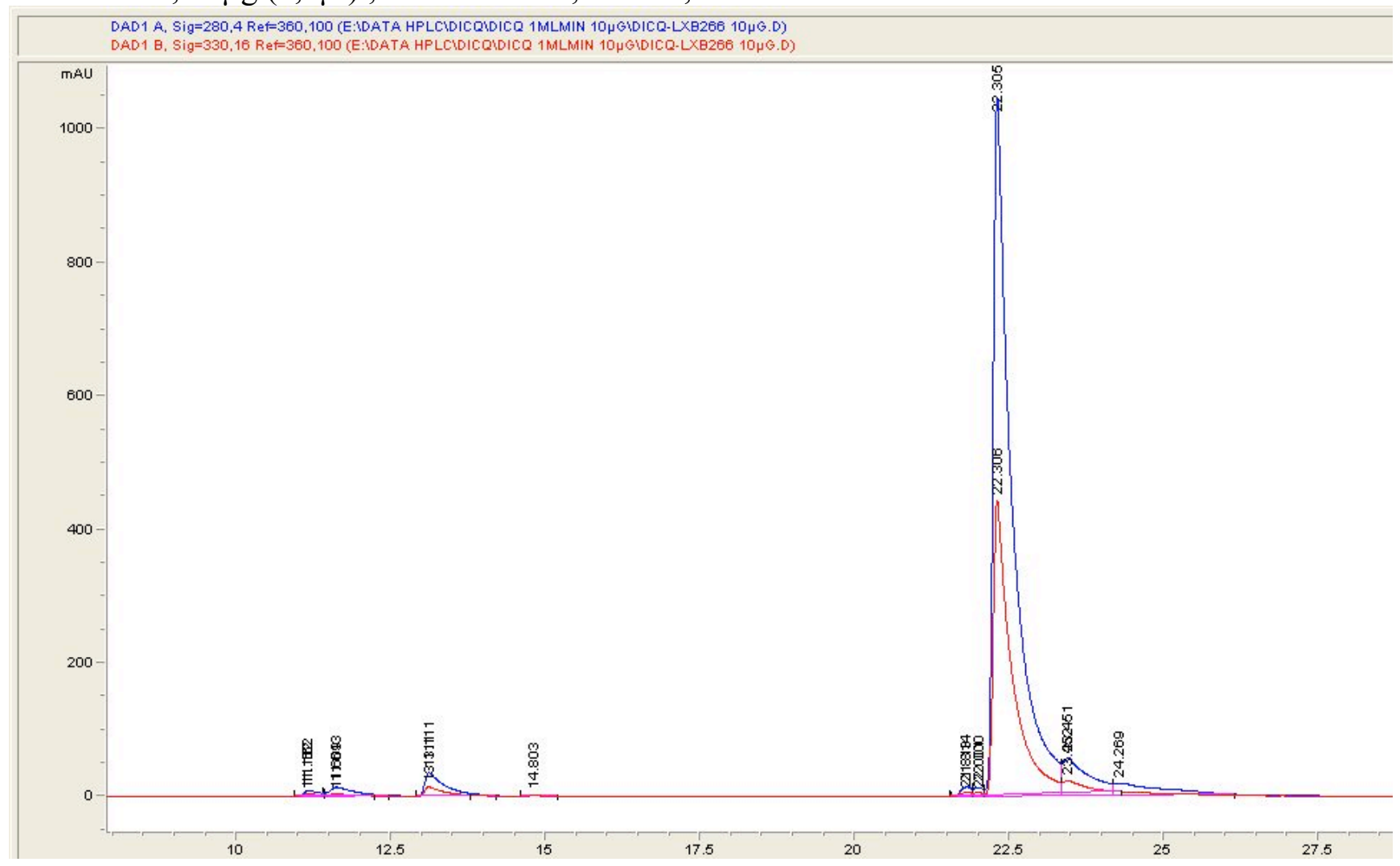

Spectre UV (210 - 400nm) à 22,308min (signal 330nm)

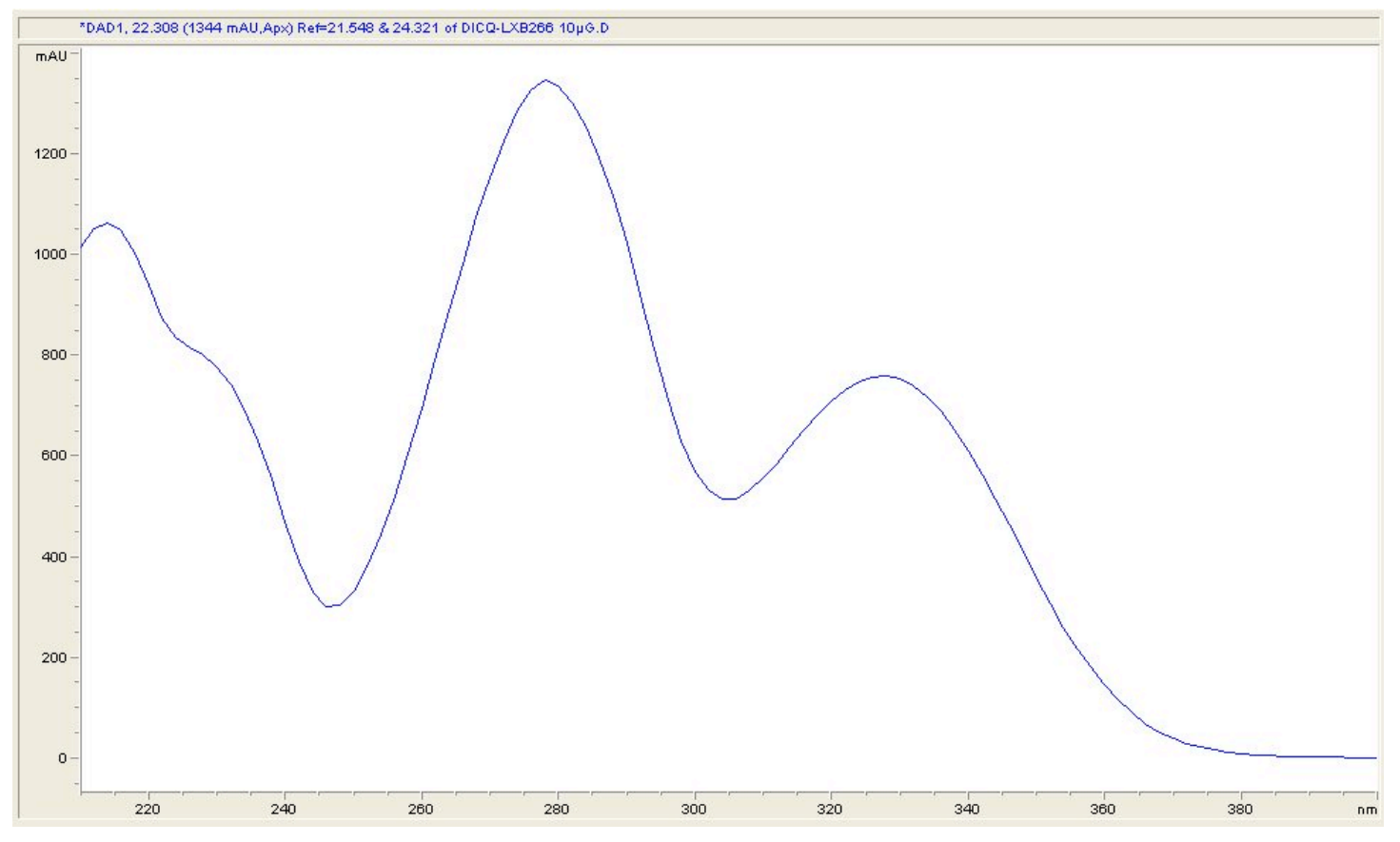


- $\quad$ 10e; $10 \mu \mathrm{g}(3 \mu \mathrm{l}) ; 270-330 \mathrm{~nm} ; \mathrm{TR}=19,4 \mathrm{~min}$

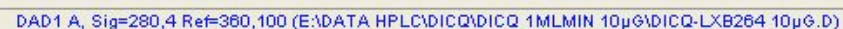

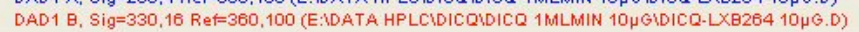

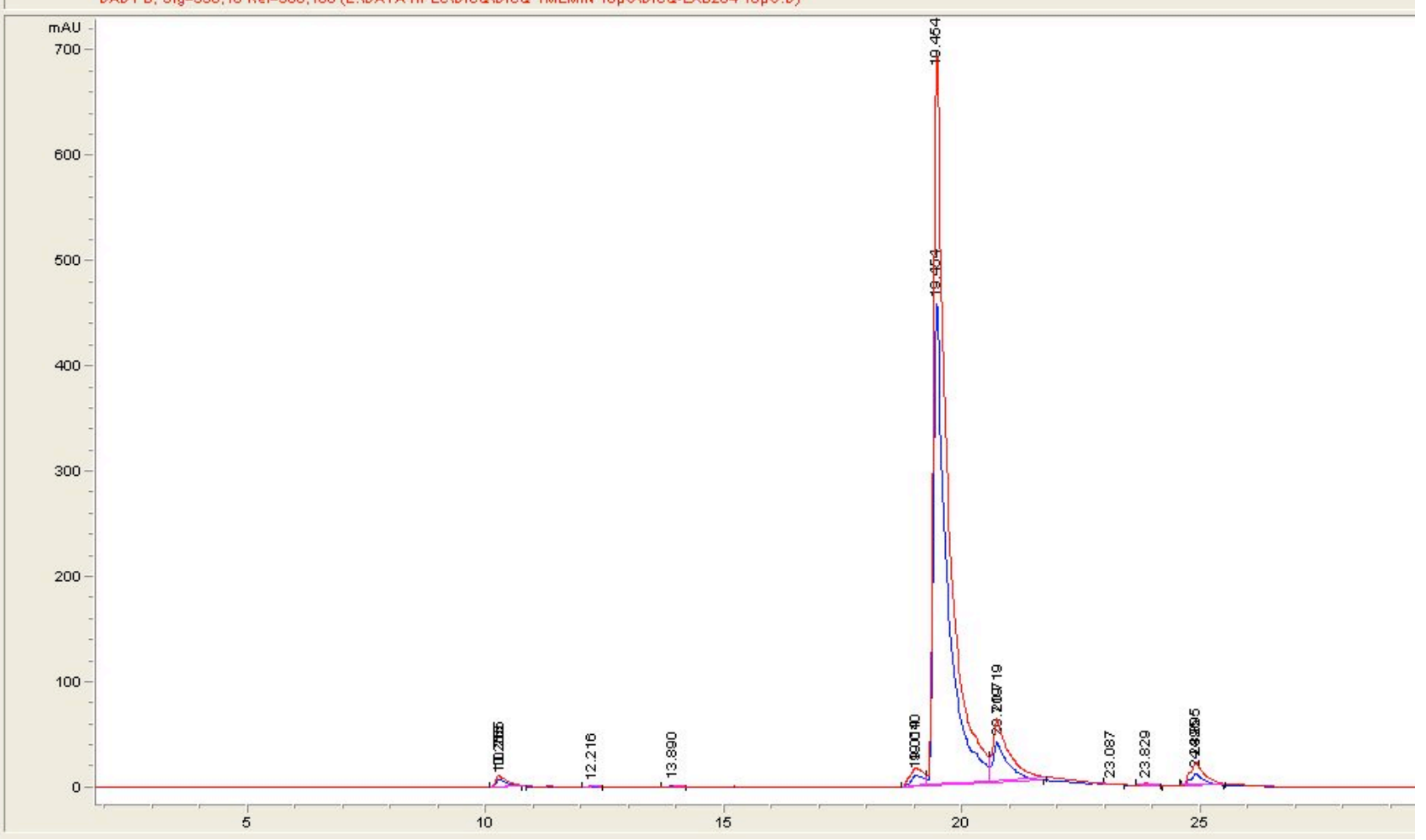

Spectre UV (210 - 400nm) à 19,569min (signal 330nm)

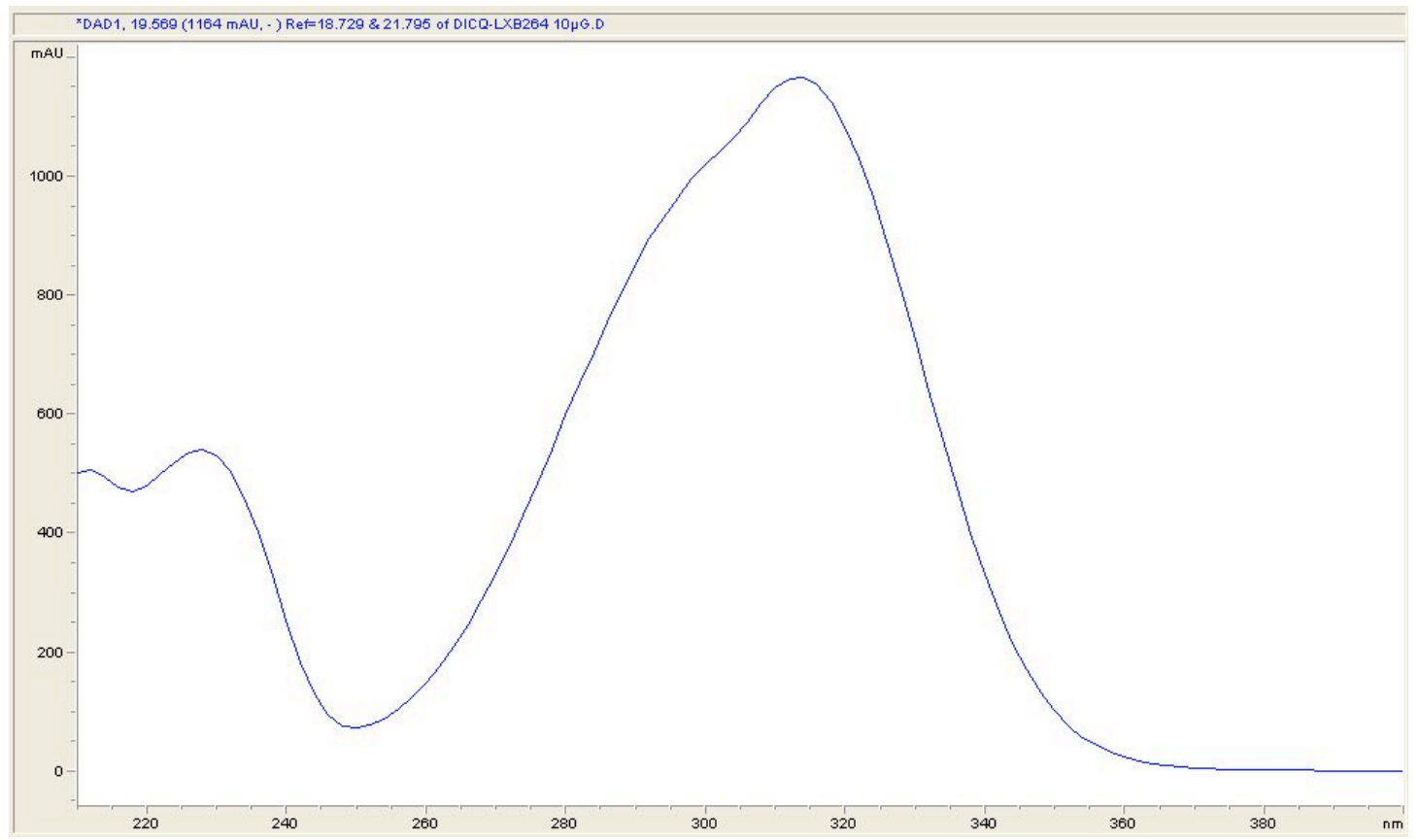


- $\quad$ 4a; $10 \mu \mathrm{g}(3 \mu \mathrm{l}) ; 270-330 \mathrm{~nm} ; \mathrm{TR}=13,9 \mathrm{~min}$

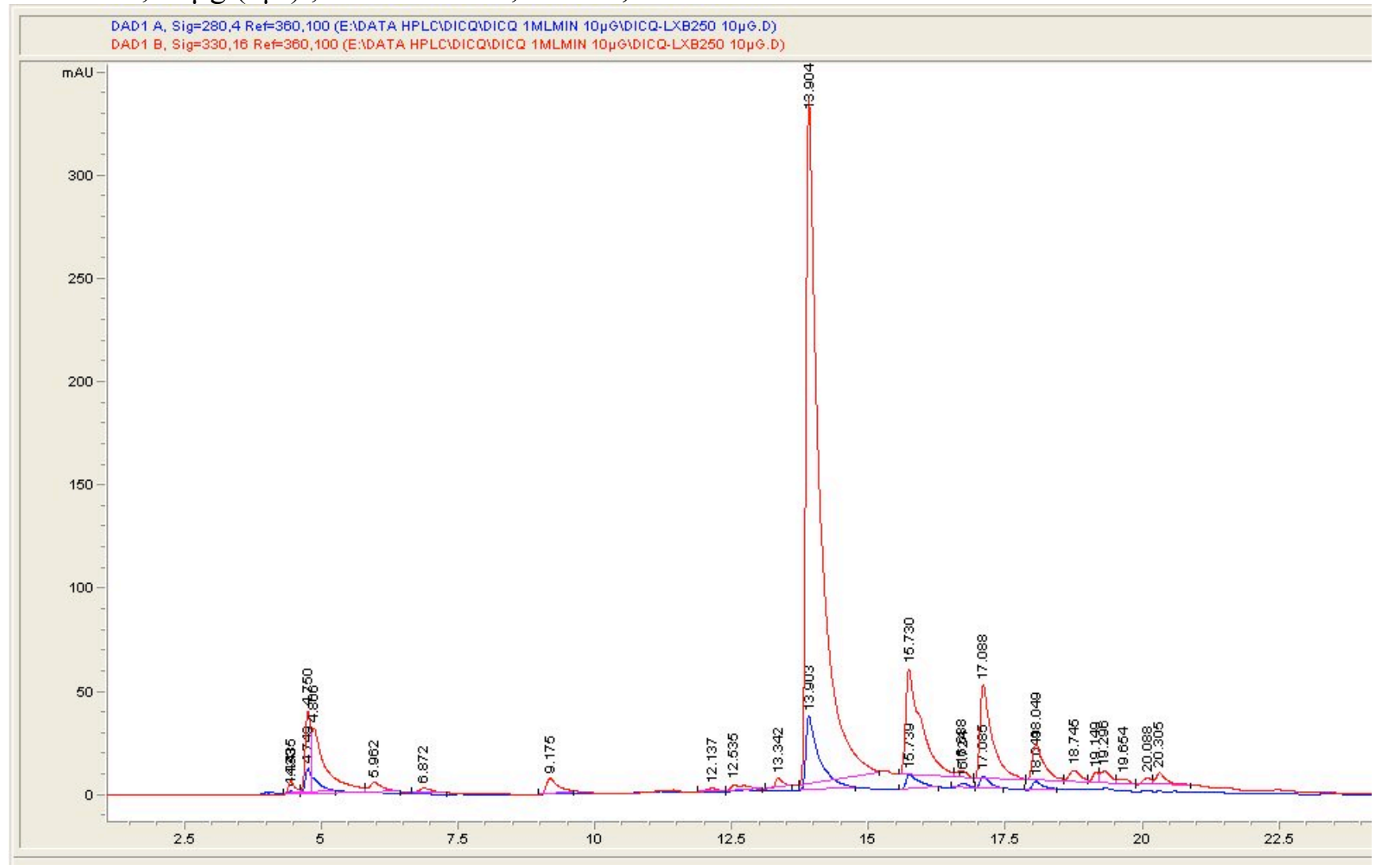

Spectre UV (210 - 400nm) à 13,910min (signal 330nm)

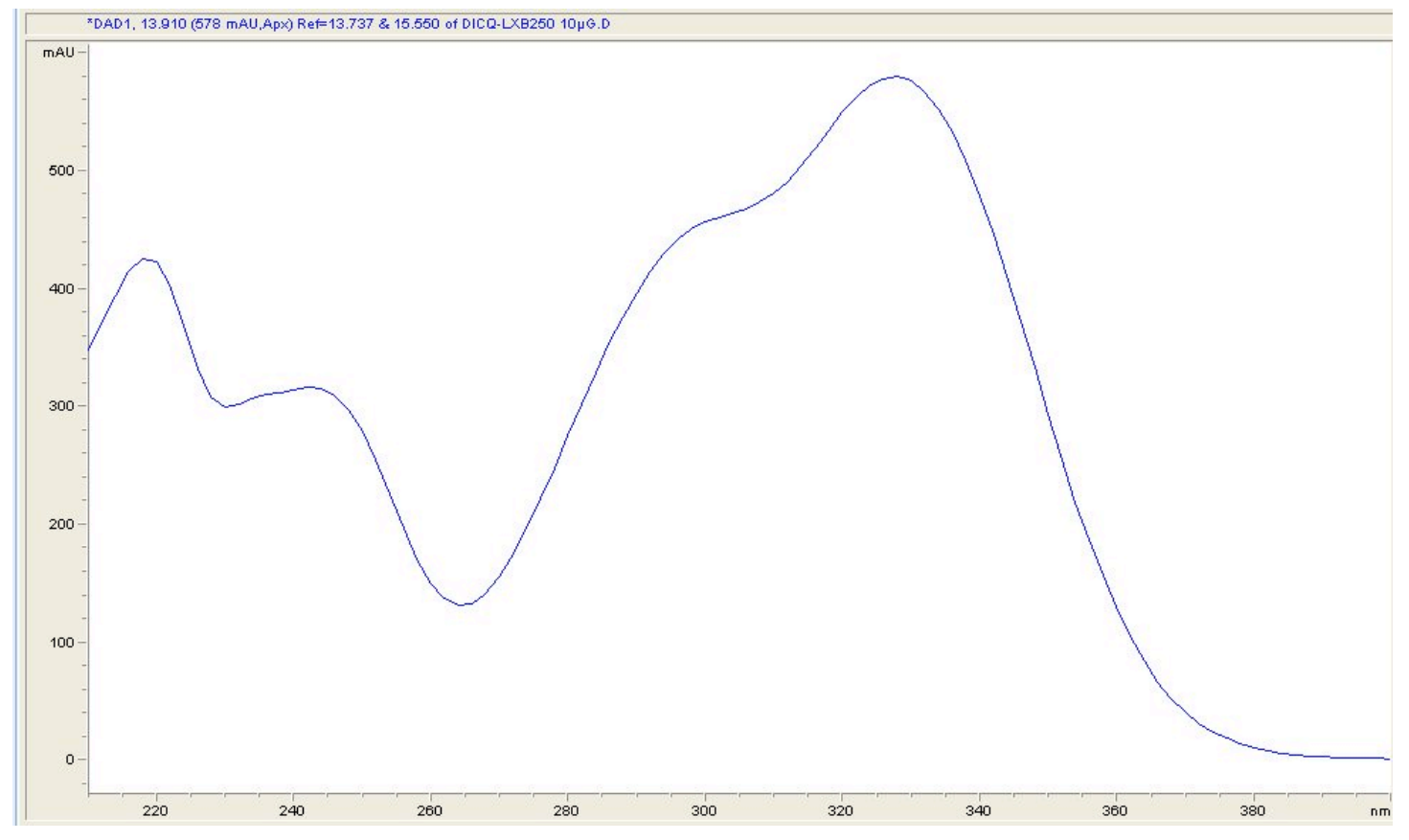


- $\quad 4 c ; 10 \mu \mathrm{g}(3,5 \mu \mathrm{l}) ; 270-330 \mathrm{~nm} ; \mathrm{TR}=17,8 \mathrm{~min}$

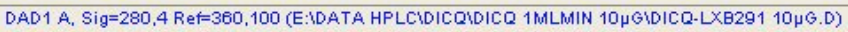

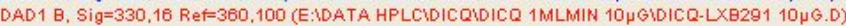

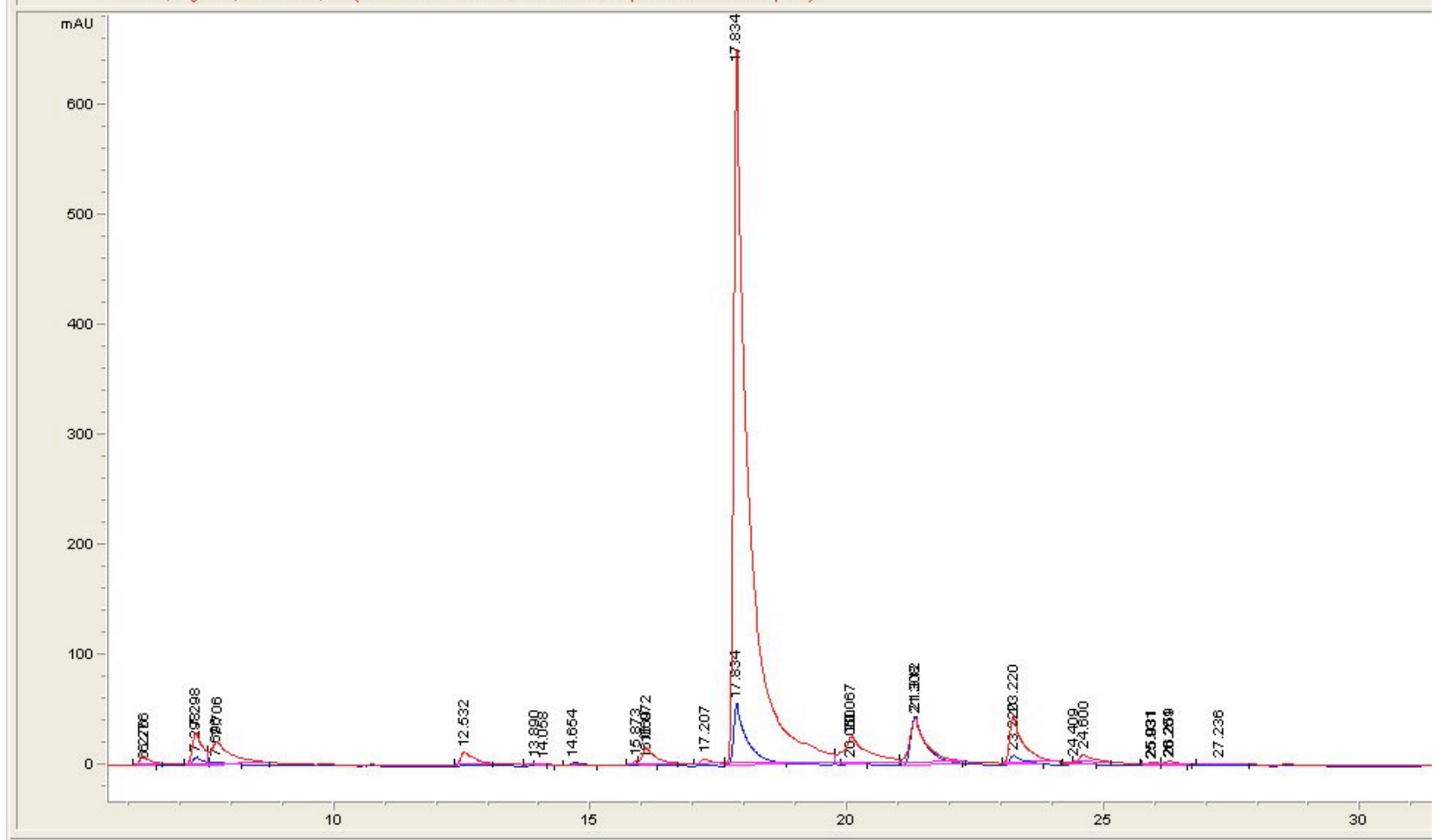

Spectre UV (210 - 400nm) à 17,823min (signal 330nm)

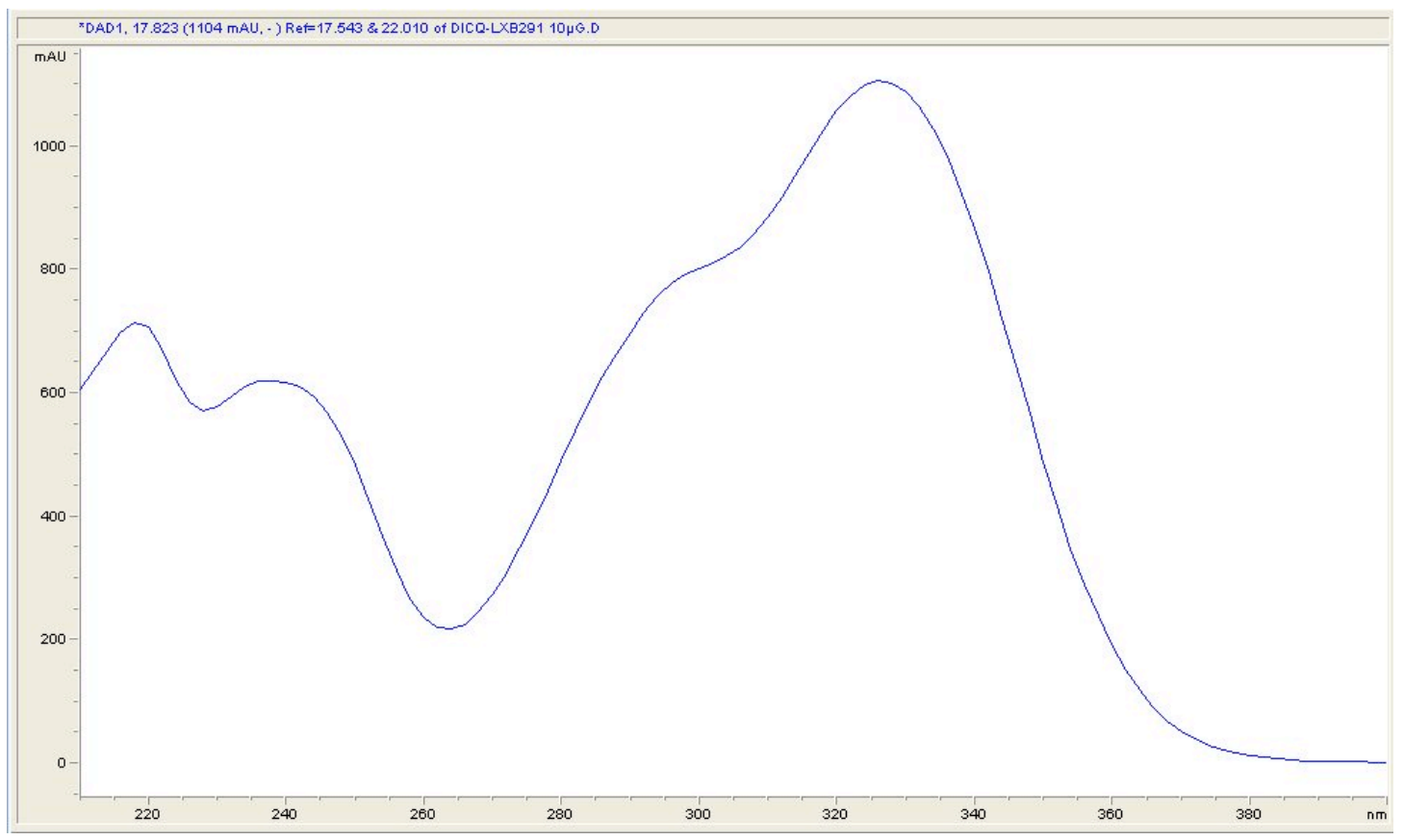


- $\quad 4 d 10 \mu \mathrm{g}(4 \mu \mathrm{l}) ; 270-330 \mathrm{~nm} ; \mathrm{TR}=17,2 \mathrm{~min}$

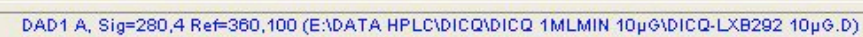

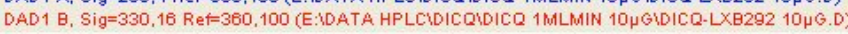

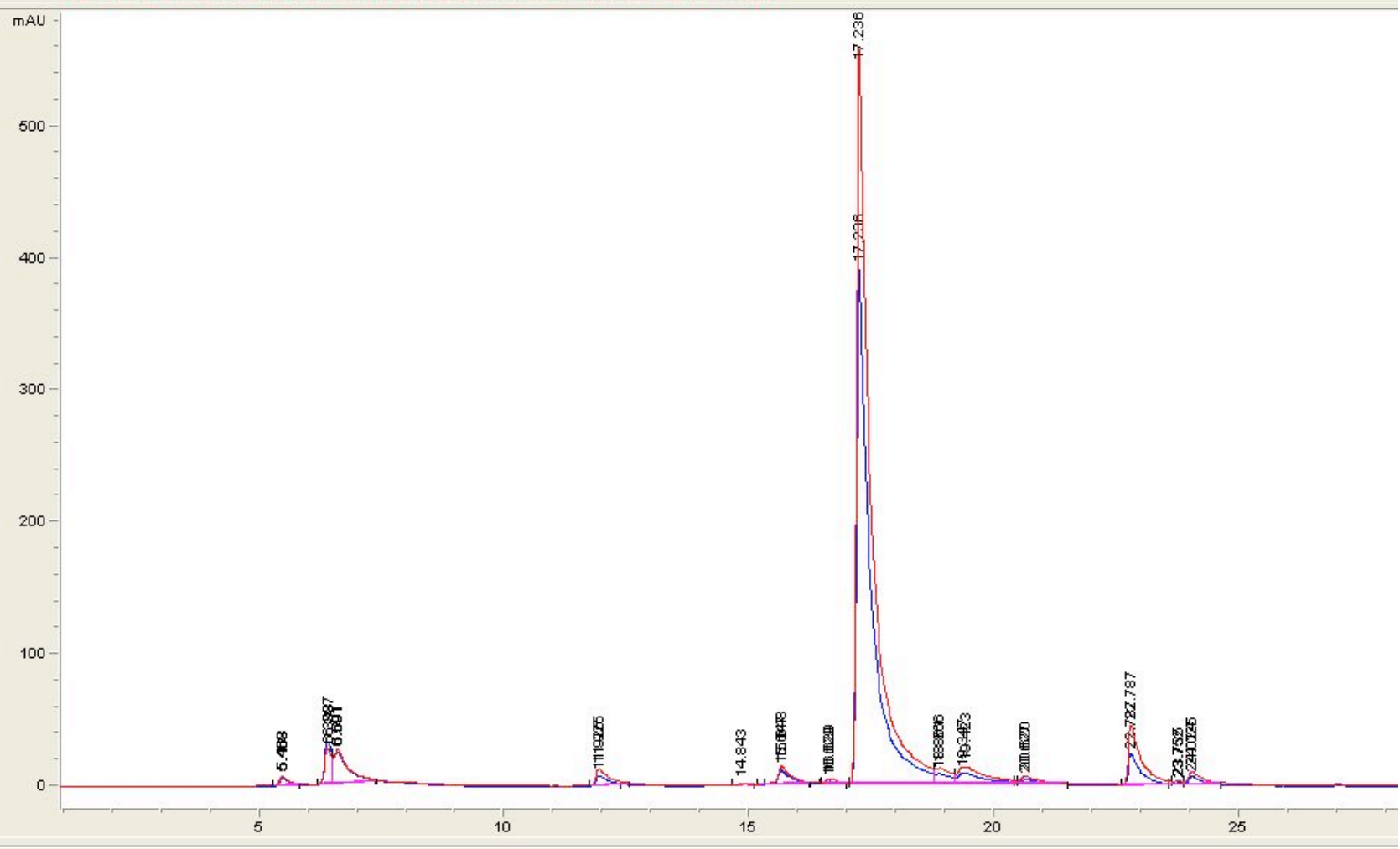

Spectre UV (210 - 400nm) à 17,207min (signal 330nm)

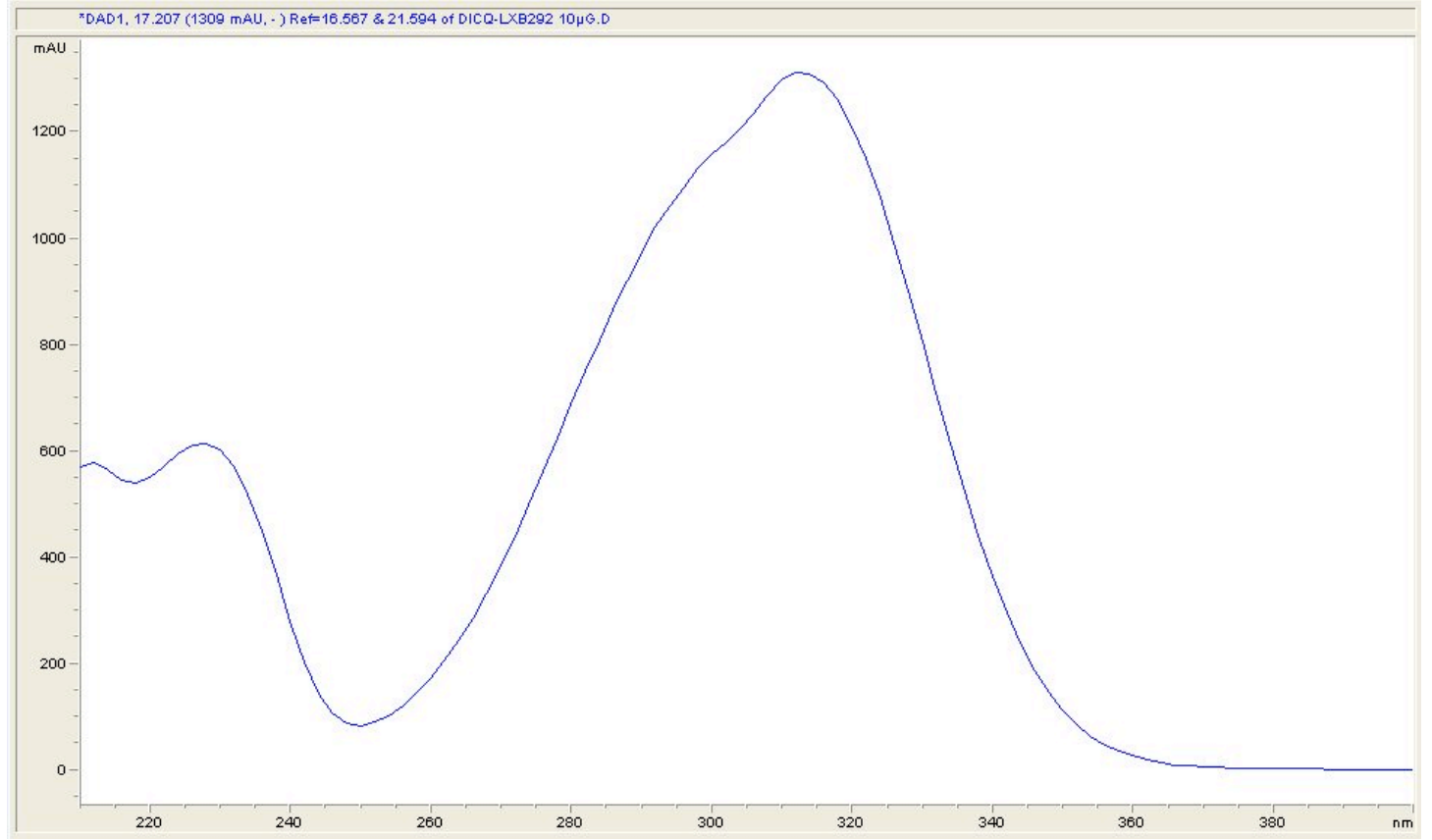


12 : (dissolved in 1,5ml MeOH); $24 \mu \mathrm{g}$ injected $(2 \mu \mathrm{l})$;

$280-330 \mathrm{~nm} ; \mathrm{TR}=16,5 \mathrm{~min}$

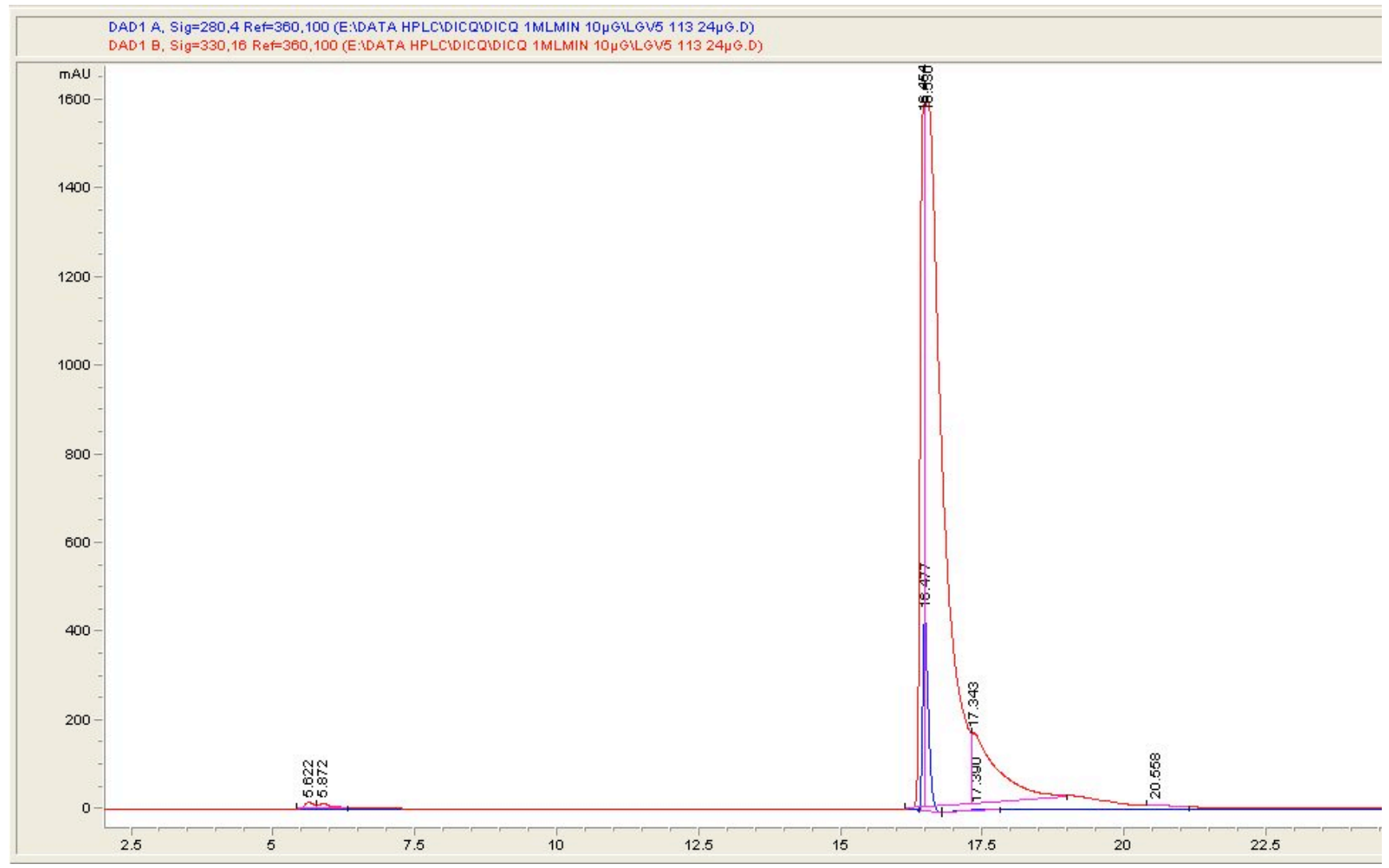

Spectre UV (210 - 400nm) à 16,374min (signal 330nm)

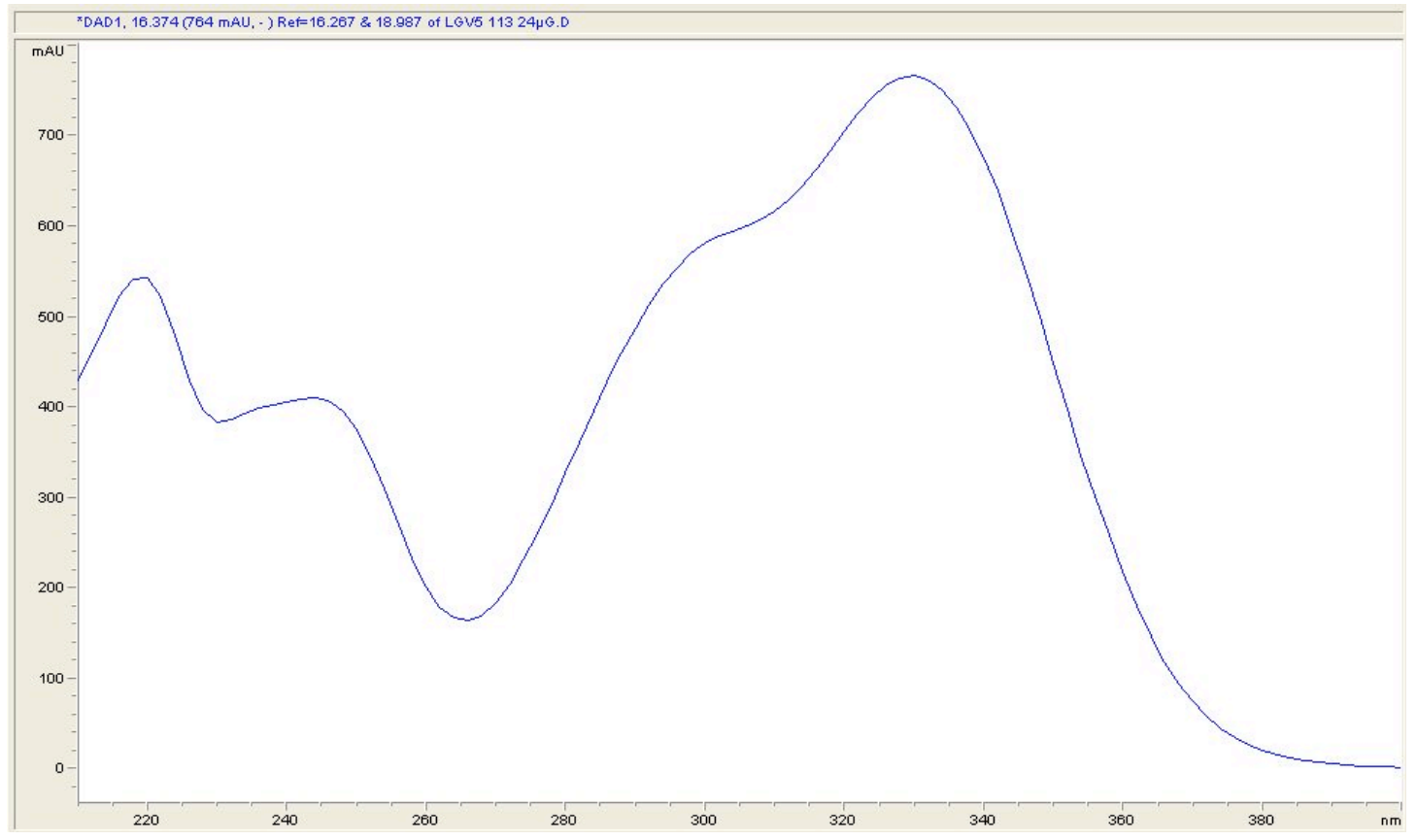


Table S3 Survival analysis ${ }^{a}$

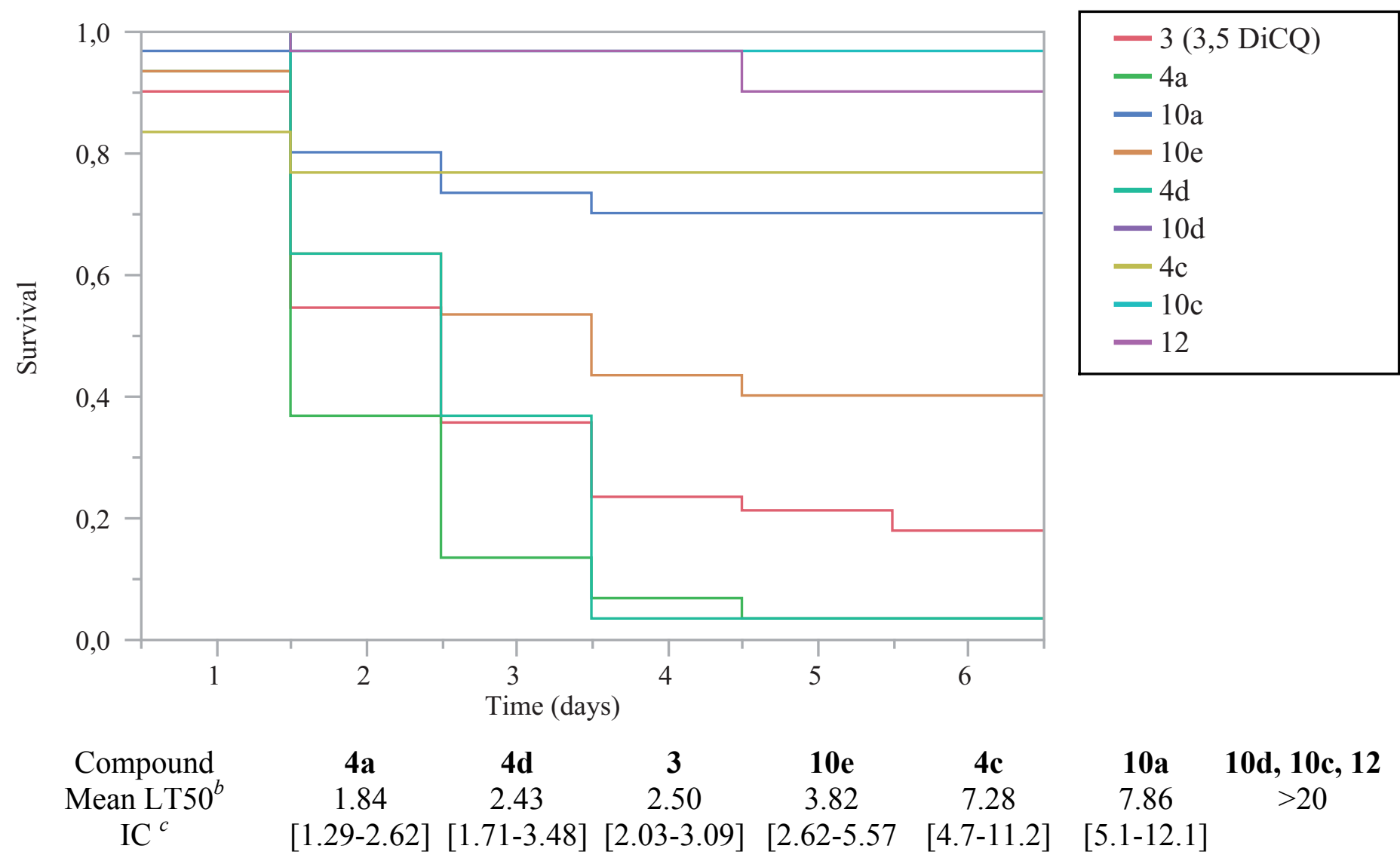

${ }^{a}$ Detailed mortality curves upon ingestion of synthetized compounds 10a to 12, incorporated in artificial diet of the pea aphid Acyrthosiphon pisum at $0.5 \mathrm{mM} ; .{ }^{\text {bin days }}{ }^{c}$ confidence intervals (at $95 \%$ confidence levels) of mean LT50 under log-normal survival model. 